HORST SIEBERT (Hrsg.)

\title{
UMWELTALLOKATION IM RAUM
}




\section{HORST SIEBERT (Hrsg.)}

\section{UMWELTALLOKATION IM RAUM}

In diesem Sammelband werden Beiträge zusammengefaßt, die sich mit der Nutzung der Umwelt in ihren verschiedenen Funktionen (öffentliches Konsumgut, Rezeptor von Schadstoffen) unter dem besonderen Aspekt der räumlichen Dimension von Umweltgütern befassen. Der Band enthält wirtschaftspolitisch und theoretisch ausgerichtete Beiträge. Insbesondere wird versucht, die Bestimmung der anzustrebenden Umweltqualität in räumlichen Systemen (Regionen) und die für die Erreichung dieser Umweltqualität einzusetzenden Instrumente zu erklären.

Udo Schneider wurde 1971 in Worms geboren und studierte von 1990 bis 1996 Volkswirtschaftslehre an der Universität Mannheim. Von 1997 bis 2001 arbeitete er als wissenschaftlicher Mitarbeiter am Lehrstuhl für Allgemeine Volkswirtschaftslehre, insbesondere Finanzwissenschaft an der Universität Greifswald. Zur Zeit ist er als wissenschaftlicher Assistent am Lehrstuhl für Volkswirtschaftslehre III, insbesondere Finanzwissenschaft der Universität Bayreuth tätig. 
Umweltallokation im Raum

Horst Siebert - 978-3-631-75607-2

Downloaded from PubFactory at 01/11/2019 03:09:22AM

via free access 


\section{STAATLICHE ALLOKATIONSPOLITIK IM MARKTWIRTSCHAFTLICHEN SYSTEM}

Herausgegeben von

Heinz König, Hans-Heinrich Nachtkamp,

Rüdiger Pethig, Horst Siebert, Eberhard Wille

Band 1

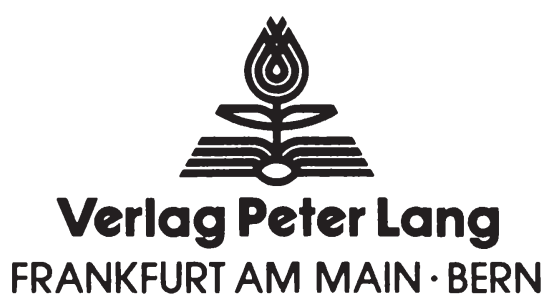




\section{HORST SIEBERT(Hrsg.)}
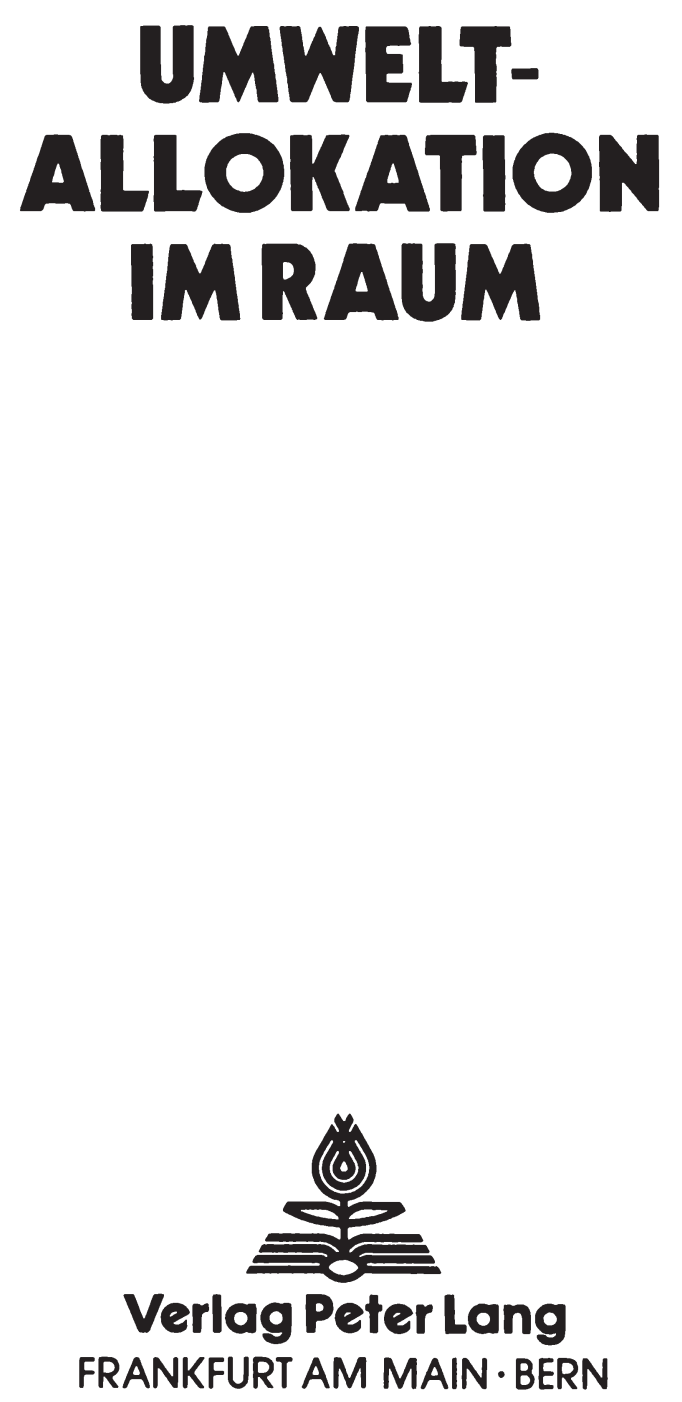

Horst Siebert - 978-3-631-75607-2

Downloaded from PubFactory at 01/11/2019 03:09:22AM

via free access 
CIP-Kurztitelaufnahme der Deutschen Bibliothek

Umweltallokation im Raum / Horst Siebert (Hrsg.). -

Frankfurt am Main ; Bern : Lang, 1982.

(Staatliche Allokationspolitik im marktwirt= schaftlichen System ; Bd. 1)

ISBN 3-8204-5997-9

NE: Siebert, Horst [Hrsg.]; GT

Open Access: The online version of this publication is published on www.peterlang.com and www.econstor.eu under the international Creative Commons License CC-BY 4.0. Learn more on how you can use and share this work: http://creativecommons.org/licenses/ by/4.0.

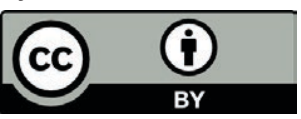

This book is available Open Access thanks to the kind support of ZBW - Leibniz-Informationszentrum Wirtschaft.

Diese Arbeit ist im Sonderforschungsbereich 5 der Universität Mannheim entstanden und wurde auf seine Veranlassung unter Verwendung der ihm von der Deutschen Forschungsgemeinschaft zur Verfügung gestellten Mittel gedruckt.

ISSN $0721-2860$

ISBN 3-8204-5997-9

ISBN 978-3-631-75607-2 (eBook)

(C) Verlag Peter Lang GmbH, Frankfurt am Main 1982

Alle Rechte vorbehalten.

Nachdruck oder Vervielfältigung, auch auszugsweise, in allen Formen wie Mikrofilm, Xerographie, Mikrofiche, Mikrocard, Offset verboten.

Druck und Bindung: fotokop wilhelm weihert KG, darmstadt 
Vorwort

Der vorliegende Band faßt einige Arbeiten des Sonderforschungsbereiches 5 "Staatliche Allokationspolitik im marktwirtschaftlichen System" an der Universität Mannheim zum Problem der Umweltallokation im Raum zusammen. Dabei wird die Nutzung solcher Umweltgüter behandelt, die sich - wie etwa Flußsysteme oder Luftbecken - auf räumliche Teileinheiten einer Volkswirtschaft beziehen. Es zeigt sich, daß die in der Umweltpolitik allgemein für eine Volkswirtschaft diskutierten Probleme durch den Bezug auf Regionen recht konkret werden und interessante Aspekte der Umweltnutzung beleuchten.

In dem einleitenden Beitrag "Environmental Policy Instruments. Some Open Questions" (Siebert) wird übersichtsartig über einige Erfahrungen mit den wichtigsten umweltpolitischen Instrumenten berichtet. Die Implikationen der direkten Kontrollen für die Ansiedlung von Firmen in Regionen und den Markteintritt, die Suche nach flexibleren Formen der Regulierung ("Bubble"-Konzept), transferierbare Emissionsrechte und die Kostenaufschlüsselung in der Wassergütewirtschaft haben direkten Bezug zur regionalen Dimension der Umweltpolitik.

Die Bezüge zwischen einer auf Teilräume ausgerichteten Umweltpolitik und der Regionalpolitik werden im zweiten Kapitel "zur Umwelt- und Regionalpolitik in der Bundesrepublik Deutschland, Überlegungen zur Raumplanung, regionalen Förderung und Luftreinhaltepolitik" (Vogt) behandelt. Im einzelnen wird dabei auf den Stellenwert des Umweltschutzes im Raumordnungsrecht der Bundesrepublik Deutschland eingegangen sowie die Vereinbarkeit zwischen der Praxis der bundesrepublikanischen Politik der regionalen Wirtschaftsförderung und umweltpolitischen Zielvorstellungen untersucht. Auch die auf der Grundlage des Bundesimmissionsschutzgesetzes betriebene Luftreinhaltepolitik wird hinsichtlich ihrer regionalpolitischen Implikationen einer kriti- 
schen Betrachtung unterzogen.

Nach diesen eher auf die praktische Umweltpolitik bezogenen Beiträgen werden im zweiten Teil des Buches Fragen der Bestimmung der Umweltqualitätsziele diskutiert.

Ausgehend von einem Flußsystem, wird in dem Beitrag "The value of Environmental Quality" (Dehez) die Frage gestellt, wie das Optimum der Umweltpolitik $\mathrm{zu}$ bestimmen ist. Es wird ein theoretischer Rahmen entwickelt, der es gestattet, die Nutzen der Wassergütewirtschaft $\mathrm{zu}$ erfassen. Zugleich werden die verschiedenen Ansätze zur Ermittlung der Zahlungsbereitschaft (direkter versus indirekter Ansatz) dargestellt.

Die Festlegung der Umweltqualitätsziele bereitet deshalb Schwierigkeiten, weil bei diesem öffentlichen Gut Wirtschaftssubjekte die Position des Freifahrers einnehmen können und ihre Präferenzen nicht "wahr" offenbaren. Wird Umweltqualität als ein regionales Gut interpretiert, so läßt sich das Tiebout-Theorem auf die Frage nach der Ermittlung der Präferenzen anwenden, wobei Wirtschaftssubjekte ihre Präferenzen durch Wanderungsbewegungen aufdecken. In dem Beitrag "Die Allokation öffentlicher Güter bei Konsumentenmobilität" (Dudenhöffer/Gebauer) wird das Tiebout-Theorem vorgestellt. Einige seiner Prämissen werden erörtert, und es wird die Frage gestellt, durch welche Eigenschaften ein Tiebout-Gleichgewicht gekennzeichnet werden kann.

Mit der Frage nach der Aufdeckung der Präferenzen setzt sich auch der Artikel "Mehrheitswahl als Instrument regionalisierter Umweltpolitik" (Dudenhöffer) auseinander. Dieser Beitrag stellt einen Erklärungsansatz für das zustandekommen umweltökonomischer Allokationen in "demokratischen" System vor. Unter "demokratischen" Systemen werden dabei ökonomien verstanden, in denen das Ausmaß der Bereitstellung des öffentlichen Gutes "Umweltqualität" durch eine Mehrheitswahlentscheidung ermittelt wird und private Güter über Märkte allokiert werden. Im Gegensatz 
zum Tiebout-Ansatz wird hier von der Immobilität der Konsumenten in einem Zwei-Regionen-Modell ausgegangen; die Regionen sind durch interregionale Schadstoffdiffusionen miteinander verknüpft.

Die bisher angesprochenen Beiträge des Buches kreisen um die Umweltnutzung im Raum, betrachten das Problem jedoch aus der unterschiedlichen Perspektive der praktischen Erfahrung mit Instrumenten und der Aufdeckung der Präferenzen. Im dritten Teil wird der Allokationsaspekt explizit für den Zwei-RegionenFall betrachtet. In dem Beitrag " $\mathrm{Zur}$ intertemporalen regionalen Umweltallokation" (Gebauer) wird die intertemporale Nutzung von Umweltsystemen erörtert. In dem Modell sind einseitige und wechselseitige Spillovers zugelassen; die sich bei autonomer Wohlfahrtsmaximierung der Regionalbehörden ergebenden Zeitprofile der Schattenpreise für Emissionen und der Allokationsmuster werden abgeleitet und erörtert.

Mein besonderer Dank richtet sich an Frau Maria Bednarek, die weite Teile des handschriftlichen Manuskripts mit großem Geschick und viel Geduld übertragen hat. Außerdem danke ich Frau Marion Börresen und Frau Sabine Wolter für ihre sorgfältig ausgeführten Schreibarbeiten. Dank schulde ich auch Herrn Dr. Wolfgang Vogt fiir die umsichtige redaktionelle Gesamtleitung.

\author{
Horst siebert
}


Horst Siebert - 978-3-631-75607-2

Downloaded from PubFactory at 01/11/2019 03:09:22AM

via free access 
I. Ansätze der praktischen Umweltpolitik 1

Horst Siebert

Environmental Policy Instruments. Some Open Questions

Wolfgang Vogt

Zur Umwelt- und Regionalpolitik in der Bundesrepublik Deutschland, Überlegungen zur Raumplanung, regionalen Förderung und Luftreinhaltepolitik

II. Bestimmung der anzustrebenden Umweltqualität

Pierre Dehez

The value of environmental quality

Ferdi Dudenhöffer / Helga Gebauer

Die Allokation öffentlicher Güter bei Konsumentenmobilität. Eine Anmerkung zum Tiebout-Theorem.

Ferdi Dudenhöffer

Mehrheitswahl als Instrument regionalisierter Umweltpolitik. Eine allgemeine Gleichgewichtsanalyse.

III. Regionale Umweltallokation in der Zeit

Zur intertemporalen regionalen Umweltallokation 
Horst Siebert - 978-3-631-75607-2

Downloaded from PubFactory at 01/11/2019 03:09:22AM

via free access 
I. Ansätze der praktischen Umweltpolitik

Horst Siebert - 978-3-631-75607-2

Downloaded from PubFactory at 01/11/2019 03:09:22AM

via free access 
Horst Siebert - 978-3-631-75607-2

Downloaded from PubFactory at 01/11/2019 03:09:22AM

via free access 


\section{Environmental Policy Instruments: Some Open Questions}

Horst Siebert

In the 1970's, environmental laws were enacted in nearly all of the industrialized nations. Experience in implementing these laws has been gathered, and some laws are up for revision (Clean Air Act in the United States in 1981). Taking into account the time-lag of environmental legislation among nations, revisions will be spread over the decade. The 1980 's will be the period of evaluating and revising the body of existing environmental laws.

This process of revision takes place in an economic situation where the energy problem requires difficult adjustments, a sizable reallocation of factors of production and shift in sector structure, where an important part of real income of the industrialized nations is transferred to the OPEC countries and where lagging productivity growth, unemployment, inflation and balance-of-payments deficits represent pressing macroeconomic policy problems. Under these conditions, the criterion of economic efficiency of environmental policy instruments receives a greater weight. Environmental policy cannot afford to be inefficient since this implies the risk that the targets of environmental policy will be reduced. Thus, in analyzing how to improve the efficiency of environmental policy instruments, the economist is the environmentalist's friend.

The environmentalist cannot rely on the hypothesis that the energy problem will present a great stimulus to resource conservation and thus to an improvement of environmental quality. This is only correct insofar as incentives to conserve resources will imply that a smaller volume of material will be withdrawn from nature, and consequently, returned to the 
environment and that changing relative prices will stimulate recycling, conservation, substitution and innovation and consequently reduce the volume of emissions. In contrast to these positive developments for the environment, there is a conflicting relationship between the energy problem and environmental protection. The pollution-intensive producing sectors of the economy (resource extraction, energy production, the chemical industry, steel, paper) are also energy-intensive. These so-called basic industries which belong to the industrial core of an economy are negatively affected by the energy problem. Energy production is capital-intensive. The increased demand for capital in energy production competes with capital requirements for abatement. Extraction of new resource deposits may involve increased environmental risks (open pit mining, sea bed mining, oil shale). Energy conservation may only be possible at the cost of a larger volume of emissions. The smaller increase in real income (in some countries even a decline) has reduced the acceptance of environmental considerations. Finally, the energy constraint has affected the political position of industrialized nations. Therefore the political acceptance of additional constraints on economic activities, established from inside, is considerably reduced.

\section{Transforming Quality Targets into Individual Behavior}

In discussing the problem of environmental policy instruments we start from the assumption that the targets of environmental policy are determined in a political process and are given. The problem then is how these targets can best be transformed into discharge and abatement behavior of individual entities. What institutional arrangements and which policy instruments are available so that the target of 
environmental protection is reached by the individual decisions of a set of polluters? We may distinguish among the following instruments.

(1) Moral Suasion. The policymaker attempts to influence the targets of private subjects in such a way that the social impact of private decisions is considered more carefully; that is, the political leader tries to change the orientation of households and producers.

(2) Subsidies. The government pays subsidies in order to induce abatement activities or reduce pollution. The subsidies are financed by general taxes.

(3) Regulation. A regulatory approach is followed in which the government specifies the maximum amount of emissions per firm or per equipment (emission norms, permits). When a quality target is violated in an environmental medium, no new permit $c$ an be issued.

(4) Emission Taxes. A price per unit of emission is charged (emission tax, effluent charge) with the intent to induce abatement or less pollution-intensive technologies.

(5) Transferable Discharge Permit. By fixing the quality target, the policymaker determines the tolerable total quantity of all emissions, that is, the sum of emission rights for an environmental medium. These emission rights are either auctioned among competing users or are sold and purchased in an artificial market.

(6) Cost-Sharing. Associations for specific environmental media are formed that efther determine the quality target themselves or implement the quality target which is specified by the policymaker. The role of these associations is to distribute the costs of achieving a desired environmental quality to the polluters; the attribution of costs should be undertaken in such a way that incentives for abatement are created. 
In the following analysis, two instruments are not considered further, namely, moral suasion and subsidies. Moral suasion is an attempt to influence the targets of private economic subjects in such a way that the social consequences of private decisions are considered. It includes a change of ethical norms with respect to nature and ecological problems. This approach may bring about results, but since the economic success of an enterprise is the central element of a free-market system, we cannot rely on firms to consider the social effects of their economic decisions. Rather, it should be the task of the economist to change the frame of reference (the data corona) of private economic decisions in such a way that social costs are internalized.

Subsidies are practical and proposed in a number of forms in environmental policy. Quite a few objections can be raised against subsidies. They have to be financed by general taxes, and in most industrialized countries subsidies already account for a large part of the budget. Also, whereas most subsidies are motivated by social policies such as health care or agriculture, the environmental problem is an allocation question. The main objection to subsidies, however, is that subsidies stimulate the pollution-intensive commodity. They take over a part of the environmental damage. Because of this subsidization, the enterprise does not need to introduce these costs into its price. Therefore, the price of the pollution-intensive commodity is too low in comparison to commodities being produced favorably to the environment. The price structure as an allocation guideline does not change as is desired. In comparison to a desired optimal situation, excessive quantities of pollution-intensive commodities should be limited. The subsidy systematically distorts the economic price mehcanism and causes a 
false allocation of resources.

In the following considerations, we will briefly review the criteria for evaluating instruments and then focus on the regulatory approach, emission taxes, discharge licenses and institutional arrangements for cost-sharing. The policy instruments have already been described in the literature in great detail (Kneese and Bower 1968; Kneese and Schultze 1975; Mills 1978). In the following discussion, we will address some of the open questions that arise in applying these policy instruments.

\section{Direct Regulation}

The regulatory approach seeks to reach a given quality target for an environmental system by regulating individual behavior. The typical instruments are pollution norms, that is, allowances to emit a specific quantity of a pollutant into an environmental system. Permits are issued until the quality target has been reached; then no further permits are given.

Regulations can take different forms according to what they specify. The usual permit is a property right to emit a maximum quantity of pollutants. Other types of regulations are obligations to reduce a given amount of pollutants, in absolute or in relative quantities. Still other examples of this approach include regulations which stipulate the state of technology to be applied in abatement or production or which monitor the type of input to be used. Product norms may define the quantity of pollutants which are contained in goods (DDT, for example, in agricultural products) or which emerge through the use of commodities (noise emitted through the use of commodities such as a car). Production quantities may be limited, or production of a specific product may be 
lower cost than firm 1 can abate $A D$. An emission tax OT shows the efficient solution.

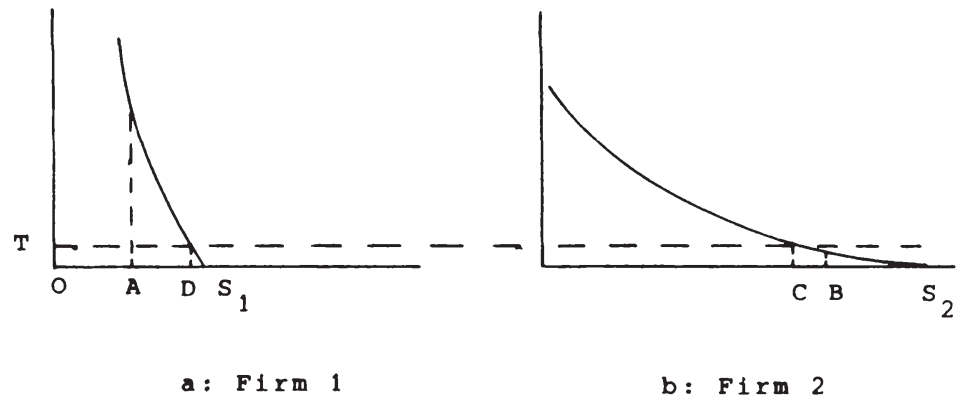

Figure 1

The inefficiency argument implies that resources are wasted. Thus, the opportunity costs of environmental policy are too high. Since the costs of environmental policy will have an effect on the target level, inefficient abatement implies less environmental quality. Therefore, the regulatory approach reduces the chances for an effective environmental policy.

(2) Bureaucracy. Government agencies have to issue permits specifying the allowable quantity of emissions for specific equipment within the firm. For instance, in the North Rhine Westfalia region of West Germany, air quality policy attempts to regulate each stationary source of emission (Dreyhaupt 1979). We may call this approach the "individual stack policy" where the government regulates each individual facility. According to Mills (1978, p. 186), 46,000 permits were issued 
in the United States for water pollution as a result of new legislation in the period from 1972 to 1976. We may doubt whether a government agency has all the necessary information to make a proper assessment in such matters. We may also note that such decisions may create an atmosphere in which government interference with individual decisions, even in other fields, becomes a widely accepted practice. Incidentally, in West Germany the time required to obtain pollution permits for facilities averages about three years.

(3) No Scarcity Price. The regulatory approach allocates pollution permits on a first come, first served basis. This is not a very feasible allocation mechanism. Some companies receive permits at a zero price; others are charged at a price of infinity, that is, this factor of production is not available.

(4) Market Entry and Dynamic Firms. The regulatory approach views the economy as being a static entity. As soon as the environmental quality standard for the region is surpassed, no new permits can be issued. Thus, direct regulation protects the existing firms and tends to perpetuate the given structure of firms and sectors in an economy. Dynamic firms cannot expand, new firms cannot enter the market. Competition is impaired. Necessary changes such as sectoral shifts, the expansion of growth industries and the reallocation of factors of production cannot take place.

(5) Spatial Structure and the Regional Labor Market. In preventing the location of new firms, the interregional migration of capital is impaired. Thus, a given spatial structure is likely to become encrusted. This consequence is not only to the disadvantage of business; it also negatively affects labor. New firms may not be able to locate in 
a region al though they may provide interesting and improved employment opportunities. The existing firms receive a monopsony with respect to the regional labor supply. They do not have to fear competitors in the regional labor market. Consequently, their incentive to compete for labor is reduced.

(6) State of Technology. Permits very often require that the producers use the existing state of technology. For instance, the air quality law in West Germany stipulates such a condition. This condition has a very interesting implication: The government will try to prove that new technologies are possible whereas the entrepreneur will use his talent to show that these new technologies are not feasible or not economical. We have feedback on the economic system. Whereas in a market economy it is the role of firms to find new technologies, given our scenario, firms will relinquish this function to the government.

(7) The Role of Courts. In most countries, government decisions can be made subject to checks by the courts. For instance, in West Germany the residents or the firms affected by a permit may go to the administrative courts on at least two levels. There are examples where a court has withdrawn a permit al ready granted by a local administration only to have a higher court reverse this decision after a year or two. Regulations give a greater role to the courts in the allocation process. But, excluding exceptional cases, allocation of resources cannot be undertaken by the courts.

\section{More Flexible Forms of Regulation}

In the command-and-control type of direct regulation, the government identifies the source of emissions and specifies for each source the 
permitted volume of emissions. Such an approach is rather strict; it presupposes that the government has all the relevant information to specify which measure has to be taken. In attempts of deregulation, it has been proposed that more flexible approaches of regulation should be used.

(1) Performance Standards. Instead of detailed compliance requirements more general performance requirements are proposed (U.S. Regulatory Council 1980). Such an approach would allow the regulated entity to find the most efficient way of complying. This would introduce an incentive for each firm to reach the given overall restriction with minimum costs. An example is EPA's "controlled trading" approach according to the bubble concept in air quality management (American Petroleum Institute 1980). Consider a firm with mutliple emission sources or a group of firms that are confronted with emission limitation requirements as specified in a state implementation plan. Under the bubble concept, firms may violate the requirement for one source if at another source there is excess emission reduction--the important condition being that the overall performance standard for the firm as a whole (or for the group of firms) is not violated. Thus, costs will be reduced.

Some restrictions for using the bubble concept have to be observed, relating to hazardous material, new source performance standards, meeting existing air quality standards, proof of the equivalence of the proposal to the state implementation plan, etc.

Performance standards have the advantage to reduce the costs of compliance. Though making the direct regulation more flexible, the approach is still embedded in a system of direct regulations such as 
state implementation plans and individual stack requirements. Also, costs of abatement are only minimized within a firm or a very limited group of firms. They should, however, be minimized over the whole economy .

(2) Banking and Offsets. According to the 1977 Amendment of the Clean Air Act, new sources entering a region in which the national ambient air quality standards are violated must offset the emissions they will create by purchasing reductions in emissions from existing sources. This provision gives the right to pollute to the existing firms but it allows the location of new firms in an area. There is an incentive for the newcomer to buy emission rights at the most reasonable price from existing polluters. Consequently, costs of abatement are reduced. Offsets may al so be "banked" so that they can be used in future trades or in bubble cases.

The advantage of this approach consists in releasing the restriction for newcomers in non-attainment regions and thus abolishing one of the handicaps of the more direct regulation. The problem lies in the definition of some standard of emissions, from which offsets are defined. If this frame of reference could be taken away, the offset approach comes pretty close to the marketable emission license.

Offsets show that firms can abate a larger volume than the agency anticipated. Therefore it may be very tempting for the agency to revise the detailed requirements. If the firms anticipate such a behavior, the long-run incentives for innovation will not be too strong. After all, the system of production norms in Eastern Europe produces exactly the same behavior of managers in state-owned firms. 
prohibited. Finally, the location of firms may be forbidden in a specific area.

The regulatory approach has been widely used in environmental policy. Thus, water and air quality management in the United States is based on a permit system (Mills 1978). In Western Europe and Japan, permits are used in air quality policy.

The advantage of the regulatory approach is seen in its ecological incidence. If the quality target is properly set and if private emitters do not violate the laws, then the quality target will be reached. This argument makes the regulatory approach very attractive to environmentalists. Unfortunately, the regulatory approach has severe shortcomings.

(1) Inefficiency. The regulatory approach requires a set of emission rules that apply to all emitters of a specific pollutant. The policymaker planes the economic subsystems by using a general approach, and thus he is not able to take into account particular differences. Therefore, the regulatory approach is inefficient. As an example, consider an obligation to reduce a given amount of pollutants by $x$ percent. We neglect the announcement effect which would clearly indicate that the level of pollutants should not be reduced before the instrument is applied (in fact, more pollution should be produced now so that one will be faced with only a relatively small reduction later on). In Figure 1, the marginal abatement costs of two firms are shown. Firm 1 has relatively unfavorable abttement costs, whereas firm 2 can abate at lower costs. If both firms have to reduce their emissions by one third, firm 1 will abate $S_{1} A$ with relatively high abatement costs, and firm 2 will abate $\mathrm{S}_{2} \mathrm{~B}$ with relatively low abatement costs. Abatement is inefficient in the sense that firm 2 can abate $B C$ of the pollutants at a Horst Siebert - 978-3-631-75607-2 
4. Emission Taxes

Allocation theory suggests that a zero price of environmental use implies a discrepancy of private and social costs, a distortion in favor of the pollution-intensive producing sector, a misallocation of resources and an overusage of the environment as a receptacle of wastes. Emission taxes or effluent fees attempt to introduce a price for environmental use. This proposal has been described extensively in the literature (Kneese and Bower 1968; Mills 1978; Siebert 1976; Tietenberg 1973). The following problems require discussion if emission taxes are to be used in practical environmental policy.

(1) Reaction of Firms. Emission taxes introduce an incentive to prevent emissions if the costs of prevention are lower than the emission tax. A firm has a set of potential adaptations at its disposal. One of the crucial effects of an emission tax is the inducement to introduce improved abatement technologies. Figure 2 summarizes the chain of reactions to an emission tax. The decisive reactions have to take place within the individual firm. Only after these adaptations have occurred, will relative prices of the economy change and will demand adjust accordingly.

The position that shifting the emission tax to the consumer will destroy the incentive is in error. As long as firms minimize costs, an emission tax will present the correct incentives. This even holds in monopoly (Siebert 1976).

(2) Tax Base. The correct tax base for an emission tax or an effluent charge is the quantity of emissions, measured in pounds or tons. In practical policy, we can expect that information problems will arise and that alternative tax bases need to be used. Figure 3 shows some tax bases. 


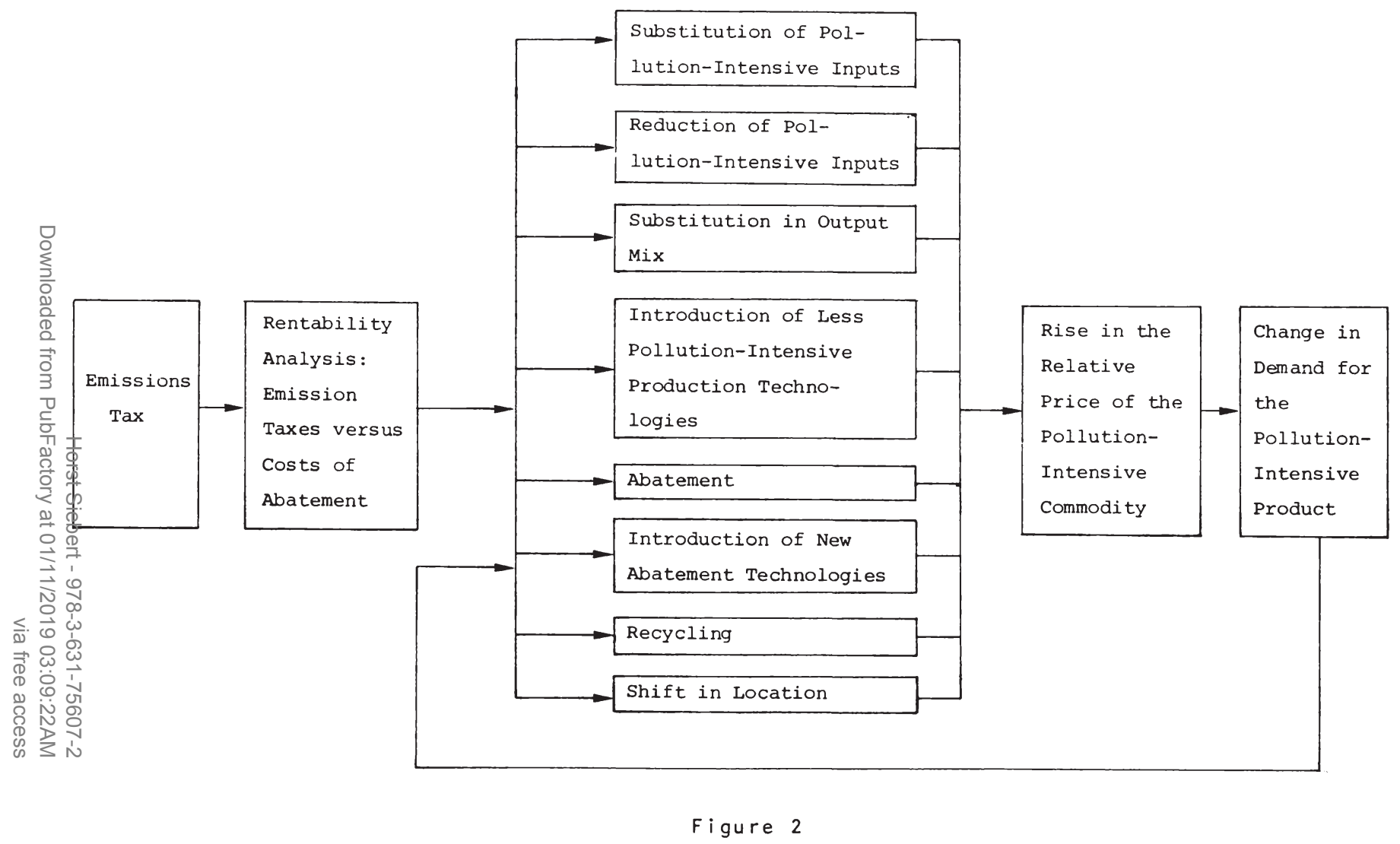




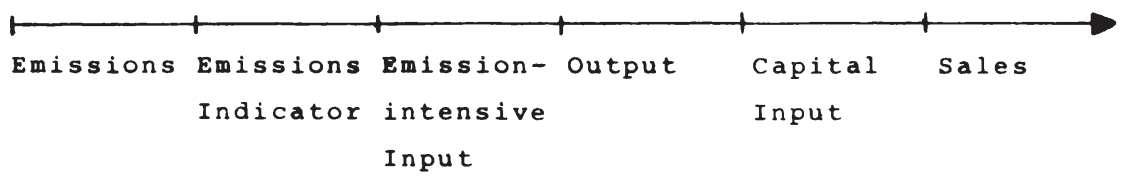

Figure 3

Assume that the quantities of emissions are not known and that we have to use proxies for emissions. Then we will not obtain the desired reactions. Let an emissions indicator such as $\mathrm{SO}_{2}$ be considered representative of all air pollutants such as $\mathrm{CO}, \mathrm{NO}_{2}$, and particulates. Then, by taxing the indicator, we stimulate abatement of $\mathrm{SO}_{2}$ but not of the other pollutants. It is quite possible that in the process of abating $\mathrm{SO}_{2}$, other emissions will be increased. A similar indicator problem arises in water quality management if emissions are calculated in units equivalent to the wastes per inhabitant. In all these cases, the indicator should be constantly revised.

If pollution-intensive inputs are taxed, we introduce an incentive to economize on these inputs; however, this target may be reached with more emissions. In this context, the problem of the second-best solution arises. Assume that we want to differentiate the tax according to a reasonable criterion such as levying a high tax rate on emissions in winter than in summer. 'If the tax base is the $\mathrm{SO}_{2}$. content of heating

11 OECD, Intruments for Controlling Sulfur Dioxide Emissions from Fuel Combustion in Stationary Sources: A Case Study of Norwegian Measures, prepared by F.F. Forsund and S. Strom (Paris, 1973), p. 23. 
0il, firms will not pollute less in winter but will buy more oil in summer and store it. Or assume that you want to use a higher tax in a metropolitan center than in the countryside. Then we will have interregional trade, and in order to maintain the tax differential, we will have to create an artificial monopoly for the oil supplier in town. ${ }^{2}$

If the tax is based on pollution-intensive outputs rather than emissions, we obtain a change in relative price and in demand. There is no response originating in the abatement and production activities. Tax bases such as capital input or sales will distort reactions even further.

(3) Measuring Emissions. It is an important question of environmental policy whether emissions can be measured within reasonable cost parameters. Note that this question also arises for the regulatory approach because, with permits, quantities of emissions are specified. Examples of measuring experience for water management can be found in Kühner (1979). Another survey of measuring technologies is given by Anderson (1977). In West Germany, the cost of measuring $\mathrm{CO}, \mathrm{SO}_{2}, \mathrm{NO}, \mathrm{HC}$, and particulates amounts to 40,000 German marks $(\$ 20,000)$ per stack and per year. 3 Self-reporting is the usual practice in monitoring emissions in the case of permits. Self-reporting, backed up by occasional checks and by measurement of the ambient environmental quality, seems to be a practical approach to the measurement problem.

(4) Interaction of Pollutants. When pollutants are diffused or when interactions such as synergisms occur, the link between emissions and quality variables seems to be destroyed. This problem, however, relates not only to emission taxes but also to regulation. We must require that the political process which establishes the ambient quality target also 2) Ibid., p. 24 .

3) Information from West German industry. 
determine the total quantity of tolerable emissions.

The point is that the quality targets are given as a result of the political process and that appropriate taxes must be found so that these targets will be reached. In setting these taxes, one must consider that an emission tax for pollutant A may lead to more pollutants of another type B. For instance, the tax for emission A may induce a new production technology with more emissions of type B. Or an emission tax may reduce emissions into the environmental medium $\alpha$, but increase emissions into medium $\beta$. Therefore, a correct vector for emission taxes has to be found so that the appropriate relative prices for different types of emissions are set.

(5) Emission Tax as a Political Price. Who will set the emission tax? One procedure is for the legislature to specify a nationally uniform tax rate. This approach has been followed in West Germany's effluent charge for water wastes. This law defines a unit of emission based on an emission indicator. Prior to 1986, 12 German marks will be charged per unit of emission; in 1986 the rate will increase to 40 German marks per unit. The law was passed in 1978 with the established tax rates being valid until 1986. Allowing for the time required to prepare and enact such laws, prices have to be fixed which will apply for a period of ten years or more.

Another procedure would be for the legislature to define the quality targets for different environmental media with respect to the most important pollutants and to transfer the right to determine emission taxes to an independent government agency. The agency would be limited by the quality targets; its role would be to set prices and adjust them in such a way that the targets would be reached. 
Such an institutional setting would be consistent with nationally uniform environmental policy instruments; it could also be applied to a regionalization of environmental policy.

\section{Transferable Discharge Permits}

In the case of transferable discharge permits, the total quantity of tolerable emissions for an environmental medium is limited in a political process. Then these emission rights are sold to those wanting to use the environment as a waste receptor. The limited quantity of emission rights is allocated through an artificial market where polluters represent demand and the government determines supply.

Discharge rights must be transferable. If a firm learns that it can abate emissions at lower costs, it must be able to sell its discharge rights to another polluter. Or if a firm wants to locate in a different area, it must be able to acquire pollution rights by inducing abatement in an existing firm. The transferability of discharge permits brings about flexibility in the allocation of the limited quantity of tolerable emissions.

This approach is beneficial because it combines the advantages of the regulatory approach with the advantages of emission taxes. By specifying the total quantity of tolerable emissions, environmental quality is clearly determined; there is no uncertainty with respect to the total quantity of emissions. In addition, a price is charged for using the environment as a waste receptor. Another advantage compared to emission taxes is that the government does not have to worry about the correct price relationship among different types of pollutants. The government only has to set the quality targets for different environmental systems. Once these quality targets are specified, the market will find the 
correct relative prices. Substitution will take place until a set of "equilibrium prices" for pollution rights is found such that demand equals supply of pollution rights.

Pollution rights may be easily used in the case of regionalized environmental policy. Assume that environmental policy sets different quality targets for regional media, for instance, in order to protect a specific area of natural beauty. Then fewer pollution rights would be supplied for this area. The price for a pollution right would be higher; consequently, either more abatement would take place, or fewer pollution-intensive sectors would locate in that region.

The following problems are connected with pollution rights (American Petroleum Institute 1980; Tietenberg 1980).

(1) Definition of Rights. Discharge permits must be defined in terms of emissions. The target variable, however, is the ambient quality of an environmental medium. The question therefore arises whether the discharge right should take into account ambient quality at the location of potential receptors. This would be the case with an "Ambient Discharge System" where each source would have to purchase receptor-specific permits. The diffusion process would have to be explicitly incorporated into the system by diffusion coefficients or other modeling approaches. This requirement, though solving the problem of interregional diffusion, is rather impractical. Consequently, as in regulation and emission taxes, the total quantity of tolerable emissions has to be specified in a political process.

The discharge right will normally be linked to a facility or a group of facilities at a location. Another proposal, emission density zoning, relates the right to the land or the landowner. After the quality target for a region has been transformed into a tolerable quantity of emissions, 
these are partitioned into quantities for zones. Then emission limitations are defined per unit of land, for instance in pounds per acre per year. The discharge right is transferable within each zone. 4

(2) Delineation of Regions. Whereas emission taxes may be used nationwide, discharge permits presuppose regional delineation of environmental media since the total quantity of rights must be defined for a specific area. Pollution rights are easier to implement for a river system than for an air system. Interregional diffusion is an important issue in this conext. If we have two regions, each with different environmental scarcity, it may be profitable to locate firms in those places of the less polluted area that are very close to the polluted region (economics of agglomeration). Thus, pollution rights may induce a spatial structure that is not desired. It may be necessary to introduce zoning in this case. Zoning may also be required if we have concentrations of pollution within an environmental region ("hot spot"). However, zoning implies that pollution rights may have to be differentiated according to zones within an environmental region. This could restrict transferability and thus would take away some of the advantages of this proposal.

(3) Complementarity in Demand. A given facility requires a set of pollution rights where pollutants normally are in a constant relation to one another. If such technical conditions are given, the transferability of pollution rights may be reduced. It is interesting to note, however, that some substitution already takes place within a firm if a firm has more than one facility.

4) In emission density zoning, the "bubble" may be too small. It may a) so be difficult to integrate the approach into the existing practice of issuing permits. 
(4) Auctioneering the Discharge Permits. One procedure for allocating pollution rights is to auction them. For instance, all pollution rights would be sold each year on a specific date in public bidding. An argument against this procedure is that firms are confronted with the risk of not receiving a discharge permit, an event which could endanger their existence. From an allocation point of view, the auction merely serves to sell and buy a factor of production. Although firms may get used to this procedure, we have to recognize that a firm usually has some certainty on the availability of factors of production such as capital, land, and labor. If the market process withdraws factors of production from a firm, it normally does so over a period of time. However, in the case of an auction for pollution rights, we may have abrupt changes. This discontinuity in the availability of a factor of production makes auctions an impractical instrument for environmental policy.

(5) Discharge Permits According to Initial Pollution. The problem of uncertainty may be prevented by giving pollution rights to the existing polluters. In this case, once could ask them to reduce pollution by a given percentage over a number of years and grant them the right to emit the residual amount. Newcomers to the region could buy a pollution right from existing firms. Although the incentive to reduce pollution would exist once this policy were implemented, there would be undesired announcement effects between the time that the measure were proposed and made effective. That is, firms would have an incentive to produce many pollutants upon learning of this policy consideration in order to receive a larger quantity of pollution rights later. Since it would take a long time to enact and possibly clarify (through the courts) such an institutional arrangement, the announcement effect may be important. 
(6) Transferability. The announcement effect can be avoided if the idea of pollution rights is combined with the regulatory approach. In the first phase, emission norms for facilities may be specified which implicitly grant a right to pollute up to a specified voluem. Then these implicitly defined rights may be made transferable. In the long run, a price for pollution rights would be established, and emission rights would be allocated via the price mechanism.

(7) Duration of Rights. Pollution rights may be defined on a temporary basis or without a time limit. If they are defined temporarily, it may be for a year or according to the life span of the facility. The allocation effects and the practicability of pollution rights may vary with these temporal definitions.

(8) Integration into Existing Laws. All environmental policy instruments have to be integrated into the existing legal framework. Very often economists make proposals that are ideal from their point of view but which do not take into consideration existing legal restrictions. In many countries, permits are used as an instrument of environmental policy. These permits specify the maximum amount of emissions allowed by a specific facility or firm per year. Very often they are granted on a temporary basis which is related to the life span of a facility. Furthermore, the permits are frequently granted at virtually a zero price. If these permits were combined with a price tag, a feasible allocation mechanism could be introduced.

(9) Monopolies. Assume that a sector of the economy happens to be located in an environmentally sensitive region. Also assume that this sector is monopolistic or has a dominant oligopolist. Then the large firm may use pollution rights to restrict the output of its competitors 
if the competitors cannot substitute emissions easily in their production. The large firm has an incentive to buy more pollution rights than it needs for production since pollution rights will not be available to its competitors. Consequently, in this case, pollution rights may strengthen the position of a dominant firm.

A similar argument is valid with respect to the labor market in a region. The large firm is induced to buy pollution rights since this may reduce the output of other firms and, concomitantly, reduce the competing demand for labor. Thus, the large firm can increase its labor supply in a region by buying pollution rights. This argument, however, is only valid if the small firms are characterized by low elasticities of substitution with respect to emissions as a factor of production (Siebert $1981)$

\section{Institutional Arrangements for Cost-Sharing}

Besides regulation, emission taxes, transferable discharge rights, a quality target can be transformed into individual behavior through a mechanism which shares the costs of reaching the targets and simultaneously develops an incentive system that guarantees efficiency. The water associations of the Ruhr area in Germany represent such an approach (Kneese and Bower 1968; Klevorick and Kramer 1973).

(1) Transforming Quality Targets. The water associations of the Ruhr area (Ruhr, Emscher, Lippe, Wupper, Niers, Erft, Left Lower Rhine, and Ruhr Water Dam Association) represent organizations in which membership is mandatory for every polluter. The general assembly of the association determines the water quality to be attained. When the required environmental quality level is known, the association can determine the 
amount of capital equipment, investment, and operating costs that it must spend to attain these standards. Thus, the total costs of abatement are specified. The problem then consists of allocating these costs to the individual polluter. Costs are attributed in such a way that the costs to the individual polluter are related to its quantity (and quality) of pollution. This creates an incentive to abate pollutants.

(2) Attributing Costs. For instance, the "Emschergenossenschaft" has developed an index that defines the quantity of unpolluted water necessary to dilute polluted water to the level where damage to a test fish is prevented. By this method, a quality target can be fixed; at the same time, different types of pollutants can be expressed in a homogeneous dimension. The formula is (Kneese and Bower 1968, p. 250; Johnson and Brown 1976, p. 123):

$v=\frac{S}{S^{Z}}+\frac{1}{2} \frac{B}{B^{Z}}+\frac{K-30}{2 K^{Z}}-F$

where $V$ is the dilution factor, $S$ the materials subject to sedimentation in centimeters per liter, $S Z$ the permitted $S, B$ the biochemical oxygen demand $\mathrm{BOD}_{5}$ in milligrams per liter after sedimentation, $\mathrm{B}^{\mathrm{Z}}$ the permitted $\mathrm{BOD}_{5}, \mathrm{~K}$ the potassium permanganate oxygen $\left(\mathrm{KMNO}_{4}\right)$ used, $K^{Z}$ the permitted $K$ in milligrams per liter, and $F$ a coefficient of fish toxicity. Let $V_{i}$ be the dilution factor for polluter $i$, and let $E_{i}$ be the be the quantity of wastewater. Then the cost share $\pi_{i}$ is given by $\pi_{i}=\frac{V_{i} E_{i}}{\sum_{1}^{n} V_{i} E_{i}} \quad$ with $\sum_{1}^{n} \pi_{i}=1$

If total costs are denoted by $C$, then the cost share for the individual 
producer is given by $C_{i}=\pi_{i} C$. The polluter $c$ an influence $C_{j}$ by reducing $\pi_{i}$, that is, by reducing $V_{i}$ and $E_{i}$. Thus, there is an incentive to reduce pollution.

The Ruhr Association uses population equipvalents (PEs) as a measure of pollution (Kneese and Bower 1972, p. 60). Dividing the total costs of abatement by the sum of all population equivalents $P E$, the price $p$ per population equivalent is obtained: $p=C \sum P E$. The cost share for the individual polluter is given by $c_{i}=p P E_{i}$.

For industrial polluters, the quantity of population equivalents is determined as follows. First, a coefficient of $0.5 \mathrm{PE}$ is used per employee. Second, wastewater is evaluated with $0.01 \mathrm{PE} / \mathrm{m}^{3}$. Third, special coefficients are used for specific sectors. For instance, 0.85 $\mathrm{PE} /$ ton of paper is the coefficient used in paper sulfide production; other examples of coefficients include $31 \mathrm{PE} /$ ton of sulfuric acid used in metal finishing or $0.35 \mathrm{PE} /$ ton of raw cabbage used in the production of sauerkraut. The coefficients vary for the firms within an industry, depending on the production and abatement technology used. For instance, for metal finishing, the coefficient varies between 31 and $6 \mathrm{PE} /$ ton of sulfuric acid used (Kühner 1979). Thus, an incentive is introduced to abate pollutants.

(3) Institutional Constraints. There are some interesting institutional features of the water associations. Voting rights vary with the volume of effluent charges paid and consequently with the volume of pollution produced; thus, the largest polluter has the greatest number of votes. In spite of this rule, analysis shows that the decisions of the associations seem to have been reasonable. Klevorick and Kramer (1973) have researched this problem and have shown that most 
environmental concerns have been taken care of by the associations. One reason for this success is that institutional safeguards have been introduced. For instance, in the Niers Association, the downstream polluters receive 75 votes before the remaining 225 votes are distributed according to the paid effluent charges. In the Lippe Association, coal mines cannot have more than 40 percent of the votes.

(4) Cost Sharing in Air Quality Management. It is tempting to ask the question whether institutional mechanisms are conceivable for cost sharing in air quality management. Performance standards for a firm with multiple emission sources or a group of firms are a step in this direction. Applying the "bubble concept", controlled trading could be organized by a voluntary association of firms or even by an "offset exchange" where firms would offer and purchase offsets.

One could even conceive of an air quality authority being made up of the polluters of a region and being confronted with an ambient air standard set by the political process. Similarly as the water associations, the air quality association would have to find a mechanism by which the costs for achieving a quality target would be minimized for the region as a whole.

The analogy between a water association and an air quality association is not perfect since in water management, pollutants are abated after having entered an environmental medium. Thus, total costs of abatement are known and have to be portioned to the polluters. In air qality management, one would have to find another mechanism by which costs would be minimized since pollutants must be prevented before entering the medium. Also the following problems have to be solved: air regions of a nation have to be defined consistently. There is the 
tendency of regional authorities to neglect interregional diffusion. Consequently, some procedure must be found to prevent a "high stack policy" (interregional diffusion norms). The three main sources of air pollution, stationary sources of households, industry and mobile sources of traffic are rather heterogeneous. How can they be incorporated into such an institutional setting? There will be a built-in tendency for such an organization to ask for subsidies. Finally, since the polluters have an influence in specifying the measurement technology and costs attributions, there may be a tendency to define parameters in such a way that entry barriers for newcomers are erected so that the regional labor market is reserved for existing firms and closed to newcomers.

\section{Taxonomy of the Environmental Problem and Policy Instruments}

In this paper we have discussed four approaches which transmit environmental quality targets into the pollution behavior of industrial polluters: regulation, emission taxes, transferable discharge permits and cost sharing. We should point out that the policy instruments used vary with the problem at hand. Our discussion mainly applies to the problem where pollutants arise as a joint output of producton. If other problems prevail, different policy instruments from the ones discussed may be necessary (Siebert 1981). For instance, if pollutants are contained in products, such as DDT in agricultural goods, we may have to use product norms. A similar problem arises with respect to pharmacological products. If pollutants are introduced into the environment via new products, such as occurs in the chemical industry, emission taxes may not be effective or practical. 
Bibliography

American Petroleum Institute, Background Paper on the Use of Economic Incentives for Environmental Protection, Washington, 1980 .

Anderson, F.R., et al., Environmental Improvement Through Economic Incentives, Baltimore and London, Hopkins, 1977.

Baumo1, W.J. and Oates, W.E., The Theory of Environmental Policy, Englewood Cliffs, N.J., Prentice-HalT, T9/5.

, Economics, Environmental Policy, and the Quality of Life,

Englewood Cliffs, N.J., Prentice-Hall, T979.

Dreyhaupt, F.J., "Clean Air Plans in Air Quality Control Regions as an Instrument of Environmental Policy", in Regional Environmental

Policy. The Economic Issues, New York, Ed. by Siebert, H., Wal ter, 1. and Zimmerman, K., New York University Press, 1979, 34-47.

Johnson, R.W. and Brown, G.M. Jr., Cleaning Up Europe's Waters, New York, Praeger, 1976.

Klevorick, A.K. and Kramer, G.H., "Social Choice on Pollution

Management: The Genossenschaften", in Journal of Public Economics,

Amsterdam, North-Holland, 1973, Vol. 2, T01-146.

Kneese, A.V. and Bower, B.T., Managing Water Quality: Economics, Technology and Institutions, Battimore, The Johns Hopkins Press, 1968.

Kneese, A.V. and Schultze, C.L., Pollution, Prices and Public Policy, Washington, Resources for the Future and the Brookings Institution, 1975.

Kühner, J., Charge Systems in the FRG's Ruhr Area, Washington, 1979, mimeo.

Mills, E.S., The Economics of Environmental Quality, New York, Norton, 1978.

Rose-Ackerman, S., "Effluent Charge: A Critique", in Canadian Journal of Economics, Toronto, University of Toronto Press, 1973, VoT. 6, $512-528$.

Siebert, H., "Erfolgsbedingungen einer Abgabenlösung (Steuern/Gebühren) in der Umweltpolitik", in Ökonomi sche Probleme der Umweltpolitik, Schriften des Vereins für Socialpotitik, Band 9, BerTin, Ed. by Issing, 0., Duncker \& Humblot, 1976a, 35-64.

, (with contributions by $W$. Vogt), Analyse der Instrumente der Umwel tpol itik, Göttingen, Schwarz, 1976.

"Voerde und eine neue Umweltpolitik", Wirtschaftsdienst, Vol. $85,1978,36-40$. 
Regional Environmental Policy. The Economic Issues, New York and' London, Ed. with H., WaTter, I. and Zimmerman, K. I, New York University Press, 1979a.

, (with A. Berthoin Antal), The Political Economy of

Environmental Protection, Greenwich, Conn., JAI Press Inc., 1979b.

, "The Economic Theory of Environmental Allocation", MIT

Energy Laboratory Working Paper No. MIT-EL 80-039WP, September 1980.

, Economics of the Environment, Lexington, D.C. Heath, 1981a.

, "Emissionslizenzen, monopolistische Marktformen und die

raumTiche Abschottung von Arbeitsmärkten", Discussion Paper,

Mannheim, 1981b, mimeo.

Tietenberg, T.H., "Controlling Pollution by Price and Standard Systems:

A General Equilibrium Analysis", in Swedish Journal of Economics,

Uppsala, Almqvist \& Wikse11, 1973, VI. $75,193-203$.

, "Transferable Discharge Permits and the Control of Air

Pollution: A Survey and Synthesis", in Zeitschrift für

Umwel tpol itik, Frankfurt, Deutscher Fachverlag GmbH, 1980, Vol. 1, 4T-508.

U.S. Regulatory Council, Regulatory Alternatives, May 1980. 
Zur Umwelt- und Regionalpolitik in der Bundesrepublik Deutschland

- Überlegungen zur Raumplanung, regionalen Förderung und Luftreinhaltepolitik -

$$
\text { Wolfgang Vogt }
$$

1. Einleitung

(1) In der Bundesrepublik

- ist nach den Niederlanden die höchste Bevölkerungsdichte in der EG anzutreffen,

- hat der Industriesektor (incl. Bau) in Relation zu anderen Sektoren den höchsten Anteil am Bruttoinlandsprodukt im EG-Vergleich, während der tertiäre Bereich einen relativ kleinen Anteil aufweist. 1 )

Besiedlungsdichte, von Region zu Region variierende wirtschaftsstrukturelle Gegebenheiten und das industrielle Aktivitätsniveau sind entscheidende Faktoren für die bundesrepublikanische Knappheit an wirtschaftlich nutzbarer Fläche und assimilativen Umweltdiensten. Von daher kommt der Regional- und Umweltpolitik eine besondere Bedeutung zu.

(2) Jede dieser Politiken wird gemeinhin als Querschnittsaufgabe klassifiziert, die herausragende Koordinierungsnotwendigkeiten zwischen mehreren Fachressorts mit sich bringt. Die föderative Struktur der Bundesrepublik erfordert neben diesen Abstimmungen auf horizontaler Ebene kooperative Regelungen zwischen den mit unterschiedlichen Planungskompetenzen ausgestatteten Gebietskörperschaften (Bund, Länder, Gemeinden).

1) Vgl. OECD-Observer Nr. 97/1979, Knoepfel/Weidner (1980), S. $126 \mathrm{f}$. 
In dieser Studie werden sowohl probleme der vertikalen und horizontalen Abstimmungsprozesse als auch im besonderen umwelt- und regionalpolitische zielkonflikte diskutiert: einerseits werden die Stellung des Umweltschutzes im Raumordnungsrecht untersucht $(\rightarrow \mathrm{Ab}-$ schnitt 2) und umweltpolitische Implikationen einzelner regionaler Wirtschaftsförderungsprogramme erörtert ( $\rightarrow$ Abschnitt 3$)$, andererseits wird am Beispiel der Luftreinhaltepolitik dargelegt, wie inflexible Bestimmungen umwelt- und regionalpolitische Ineffizienzen begünstigen $(\rightarrow$ Abschnitt 4$)$.

Es wird hier eine Beschränkung auf den umweltpolitischen Bereich der Luftreinhaltepolitik nicht nur aus Platzgründen sondern auch wegen der besonderen illustrativen Bedeutung der Luftreinhaltepolitik für die Beziehungen zwischen Umwelt- und Regionalpolitik vorgenommen. 2 )

2. Der Stellenwert des Umweltschutzes im Raumordnungsrecht

(3) Dieser Abschnitt soll eine knappe übersicht über die Berücksichtigung des Umweltschutzanliegens innerhalb des raumwirksamen Planungsrechts in der Bundesrepublik vermitteln. Denn jeder mit dem Anspruch auf Praxisbezogenzeit entwickelte umweltpolitische Reformvorschlag hat die aktuell gegebenen - also auch die rechtlichen - Restriktionen für die Umweltpolitik zu würdigen, um erste Ansatzpunkte für die Durchsetzung eines Konzeptes zu finden, dessen Inspiration aus einem idealtypischen, d.h. von jeglichen institutionellen Regelungen freigehaltenen Modell stammt. Bezug

2) Die räumlichen Dimensionen der Umweltpolitik sind von dem jeweils im Vordergrund stehenden Medium abhängig. Vgl. Siebert (1978, S. $123 \mathrm{ff}), \mathrm{Kuhl}(1977, \mathrm{~S} .95 \mathrm{f})$.

Zur regionalpolitischen Bedeutung der Wassergütepolitik am Beispiel der Abwasserabgabe vgl. BFA (1976).

Die Beschränkung dieser Problemstudie auf Wechselwirkungen zwischen Regional- und Umweltpolitik bedeutet hier selbstverständlich nicht, daß strukturpolitische Aspekte gänzlich von der Betrachtung ausgeschlossen sind. Vgl. z.B. auch Stamer $(1976)$. 
genommen wird hier auf die Phase nach 1970, also den Zeitabschnitt, in dem eine forcierte Umweltpolitik in Gestalt zahlreicher legislativer Initiativen betrieben wurde. 3) 4)

1972 erfuhren die umwelt- und raumordnungsplanerischen Zusammenhänge zum ersten Mal eine nennenswert ausführliche Behandlung in der Entschließung der Ministerkonferenz für Raumordnung (MKRO) "Raumordnung und Umweltschutz". Ebenso wie in den Umweltberichten Bayerns (1972) und Nordrhein-Westfalens (1974) wird die instrumentelle Bcdeutung der Landesplanungen als vorbeugende Umweltpolitik hervorgehoben. In Fortführung des Raumordnungsberichtes der Bundesregierung von 1974 greift das Bundesraumordnungsprogramm (BROP) (1975) raumstrukturelle Aspekte des Umweltschutzes auf. Dabei werden allerdings nur allgemeine umweltpolitische Zielaussagen berücksichtigt; die zum Ausdruck kommende großräumige Gesamtkonzeption wird nämlich primär von einer auf Teilräume bezogenen Politik zur Überwindung wirtschafts- und infrastruktureller Defizite determiniert. Daß die Bewertung funktioneller Teilräume unter Vernachlässigung ökologischer Kriterien erfolgt, ist als eine derzeit gültige Restriktion für die Etablierung einer raumordnungspolitisch abgestimmten Umweltpolitik zu betrachten. 5) - In Befolgung des grundgesetzlich verankerten regionalen Gleichheitsgrundsatzes strebt das BROP die Eliminierung regional unterschiedlicher Beschäftigungsmöglichkeiten und Infrastrukturausstattungen an. Daraus erwächst ein Koordinationszwang; denn es gilt, die verschiedenen Nutzungsanforderungen an den begrenzt ökologisch belastbaren Raum durch eine räumlich-funktionale Aufgabenund Arbeitsteilung zwischen Belastungs- und Freiräumen abzustimmen.

3) Zur umweltpolitischen Debatte in der Bundesrepublik vgl. z.B. SRU (1978; S.560). - Eine kurzgefaßte Ubersicht über gesetzliche Regelungen zum Umweltschutz ist enthalten im BMI (1980).

4) Anstöße zu einer integrativen Betrachtungsweise von Umweltschutz und Raumordnung gingen bereits 1911 bzw. 1922 und 1950 vom "Zweckverband GroB-Berlin" bzw. dem Ruhrsiedlungsverband aus. - Auch bei der Diskussion anläßlich der Verabschiedung der ersten Landesplanungsgesetze in der Bundesrepublik wurde ein ökologischer Bezug hergestellt. -Siehe Kuhl (1977; S. 2628), Umlauf (1972; S. 196 f.)

5) SRU (1978), S. 560 . 
(4) Einen gesetzlichen Niederschlag haben die Umweltschutzbelange $z . B$. im Raumordnungsgesetz (ROG) des Bundes gefunden; im einzelnen enthält das ROG folgende umweltschutzrelevante Regelungen:

Während $\S 1$ ROG einen vagen Hinweis darauf enthält, bei der Raumplanung "natürliche Gegebenheiten zu berücksichtigen, weist $\S 2$ ROG mehrere unmittelbar umweltschutzrelevante Aussagen auf:

- Die räumliche Struktur der Gebiete mit gesunden Lebens- und Arbeitsbedingungen sowie ausgewogenen wirtschaftlichen, sozialen und kulturellen Verhältnissen soll gesichert und weiterentwikkelt werden; in Gebieten, in denen eine solche struktur nicht besteht, sollen Maßnahmen zur Strukturverbesserung ergriffen werden ( $\$ 2 \mathrm{Abs} .1 \mathrm{Nr} .1 \mathrm{ROG}$ );

- Es soll eine Verdichtung von Wohn- und Arbeitsstätten angestrebt werden, die dazu beiträgt, räumliche strukturen mit gesunden Lebens- und Arbeitsbedingungen sowie ausgewogenen wirtschaftlichen, sozialen und kulturellen Verhältnissen zu erhalten, zu verbessern oder zu schaffen ( $\$ 2$ Abs. 1 Nr. 2 ROG);

- Es sind die räumlichen Voraussetzungen dafür zu schaffen und $z u$ sichern, daß die land- und forstwirtschaftliche Bodennutzung als wesentlicher Produktionszweig der Gesamtwirtschaft erhalten bleibt; die Landeskultur soll gefördert werden; für die landwirtschaftliche Nutzung gut geeignete Böden sind nur in dem unbedingt notwendigen Umfang für andere Nutzungen vorzusehen; das gleiche gilt für forstwirtschaftlich genutzte Böden ( $§ 2$ Abs. 1 $\mathrm{Nr} .5 \mathrm{ROG})$;

- In Verdichtungsräumen mit gesunden räumlichen Lebens- und Arbeitsbedingungen sowie ausgewogener Wirtschafts- und Sozialstruktur sollen diese Bedingungen und strukturen gesichert und, soweit nötig, verbessert werden; der Verdichtung von Wohn- und Arbeitsstätten soll entgegengewirkt werden, soweit sie zu ungesunden räumlichen Lebens- und Arbeitsbedingungen sowie zu unausgewogenen Wirtschafts- und Sozialstrukturen führt; wo solche un- 
gesunden Bedingungen und unausgewogenen Strukturen bestehen, soll deren Gesundung gefördert werden; die Maßnahmen zur Erreichung dieser ziele sollen auch der Erhaltung der den Verdichtungsräumen zugeordneten Landschaft dienen ( $\S 2$ Abs. 1 Nr. 6 ROG);

- Für die Erhaltung, den Schutz und die pflege der Landschaft einschließlich des Waldes sowie die Sicherung und Gestaltung von Erholungsgebieten ist zu sorgen; für die Reinhaltung des Wassers, die Sicherung der Wasserversorgung und die Reinhaltung der Luft sowie für den Schutz der Allgemeinheit vor Lärmbelästigung ist ausreichend sorge $\mathrm{zu}$ tragen ( $\S 2 \mathrm{Abs} .1 \mathrm{Nr} .7$ ROG).

Die Raumplanungsinstanzen sind also durch $\S 2$ Abs. $1 \mathrm{Nr} .2$ und Nr. 6 aufgefordert, Landschaftszersiedelungen $z$ u vermeiden und ökologische Ausgleichsräume für die Regeneration der Umweltbereiche Luft und Wasser zu sichern. Als ebenfalls essentiell für den durch die Raumplanung gegebenen Rahmen, der den Spielraum. der Umweltpolitik mitdeterminiert, ist $\S 2$ Abs. 1 Nr. 7 ROG anzusehen: dieser für den Gesamtraum geltende Grundsatz stellt einen der rechtlichen Eckpfeiler für die Luftreinhaltepolitik dar, die im Vordergrund dieser Untersuchung steht.

(5) Die umweltpolitische Effektivität der oben aufgeführten Bestimmungen des ROG läßt sich wegen ihrer allgemein gehaltenen Formulierungen nur vor dem Hintergrund des föderativen statsaufbaus der Bundesrepublik beurteilen.

Das ROG des Bundes hat eine rahmengesetzliche Funktion und wird von den Landesplanungsgesetzen der einzelnen Bundesländer ergänzt. Auf der Basis dieser Gesetze werden von den Gebietskörperschaften raumwirksame planungen vorgenommen, und zwar nach dem Prinzip der vertikalen Harmonisierungspflicht innerhalb des hierarchisch gegliederten Planungsgefüges "Bundesraumordnung $\rightarrow$ Landesplanung $\rightarrow$ Regionalplanung $\rightarrow$ (kommunale/interkommunale) Bau- 
leitplanung". 6)

Obwohl dem Bund vom Bundesverfassungsgericht (BVerfGE) die Vollkompetenz für Raumordnungsangelegenheiten "kraft Natur der Sache" zugestanden wurde, kann allenfalls eine de-facto- und keine de-jure-Verbindlichkeit des Bundesraumordnungsprogramms für die Länder festgestellt werden. ${ }^{7)}$

Die Länder erstellen in Komplettierung der Rahmenregelungen des ROG - auf der Grundlage ihrer jeweiligen Landesplanungsgesetze Programme bzw. Pläne zur Raumnutzung (bezeichnet als Landesentwicklungsprogramme oder -pläne bzw. Raumordnungsprogramme oder -pläne). 8) Die Landesplanungsgesetze enthalten zum Teil wiederholend die umweltschutzrelevanten Grundsätze des ROG, während einige Länder (Bayern, Rheinland-Pfalz, Schleswig-Holstein) eigene Umweltschutzleitlinien aufgestellt haben, die präziser als die entsprechenden Bundesrichtlinien sind; ${ }^{9)}$ andere Bundesländer (z.B. Hessen, Baden-Württemberg, Nordrhein-Westfalen, Niedersachsen, Saarland) nehmen in ihre Landesentwicklungsprogramme

6) Aus diesen Bezeichnungen geht hier die jeweilige flächenmäßige Ausdehnung der Planungsgebiete hervor.- Unter einer "Region" ist hier ein aus mehreren (ganzen) Kreisen bzw. Städten zusammengesetze Planungsregion $\mathrm{zu}$ verstehen; solche nach administrativen Gesichtspunkten konstruierten - Gebiete werden uneinheitlich, nach bundeslandabhängigen Kriterien gebildet.- Die Gemeinden sind bei der Bauleitplanung an das ROG, das betreffende Landesplanungsgesetz und das Bundesbaugesetz (BBauG) gebunden, d.h. ihre Bebauungsvorhaben haben mit den Zielen der Raumordnung und Landesplanung in Einklang zu stehen. - Der Planerstellungsproze $\beta$ auf Bundes-, Landes- oder Regionalebene verläuft konsensorientiert unter Beteiligung der nächstunteren Gebietskörperschaften ("Rückkopplungsmechanismus","Gegenstromprinzip") .

7) Siehe dazu Kuhl (1977), s. 42 f.

8) Diese von den Ländern aufgestellten programme werden entweder durch Gesetz verbindlich oder nehmen den Charakter von Rechtsverordnungen oder Verwaltungsrichtlinien an.

9) Vgl. Art. 2 des Bayerischen Landesplanungsgesetzes, $\S 2 \mathrm{Nr}$. 11 des Landesplanungsgesetzes von Rheinland-Pfalz und $\S 2$ des Schleswig-Holsteinischen Gesetzes über Grundsätze zur Entwicklung des Landes (Fassung vom 11.12.1973). 
weitere umweltbezogene Aussagen auf ${ }^{10)}$ - zumeist mit einem relativ starken Anwendungsbezug. 11)

Ähnlich wie bei der Bundesraumplanung bleibt aber auch für die Landesraumplanung als der zweiten Ebene der flächendeckenden Planung festzustellen, daß immer noch ein relativ grober Detaillierungsgrad der planerischen Festsetzungen vorliegt. ${ }^{12)}$ Erst die auf der dritten Stufe der Planungshierarchie vorgenommene - Regionalplanung füllt den durch Landesplanung gegebenen Rahmen

10) In diesem Kontext sei erwähnt, daß es den Ländern offensteht, in Ergänzung der jeweiligen Landesplanungen spezielle Umweltberichte und -programme (in denen wiederum besonders auf die raumbedeutsamen Belange des Umweltschutzes eingegangen wird) mit Bindungscharakter für untergeordnete Behörden zu entwerfen. Vgl. z.B. die Umweltberichte des Bayrischen Staatsministeriums für Landesentwicklung und Umweltfragen oder das Programm "Umweltpolitik in Bayern" von 1978.

11) Vgl. z.B. die Umweltaussagen in den Landesentwicklungsplänen Baden-Württembergs, des Saarlandes und besonders NordrheinWestfalens.- Der folgende - die Luftpolitik betreffende - Auszug aus dem 2. Durchführungsabschnitt 1975-78 zum Landesentwicklungsplan "Hessen'80" illustriert z.B. den hier gemeinten höheren Konkretisierungsgrad im Vergleich zu den ROG-Grundsätzen: Die Reinhaltung der Luft ist eine der dringendsten und wichtigsten Aufgaben des Umweltschutzes. Die Verschmutzung der Luft darf nicht weiter ansteigen, sondern sie muß mittels umfassender Maßnahmen wieder verringert werden. Hierzu müssen auch alle technologischen und planerisch organisatorischen Möglichkeiten zur Emissions- und Immissionsverminderung angewendet werden. Es müssen aber auch alle Rechtsmöglichkeiten bei Verstößen auf diesem Gebiet ausgeschöpft werden.

Bei allen Emittenten-Gruppen (Industrie, Verkehr sowie Haushalte und Kleingewerbe) müssen die Emissionen nicht nur nach dem Stand der Technik vermindert werden, sondern es mus auch dafür gesorgt werden, daß technische Verfahren entwickelt werden, die eine maximale Rückhaltung der Schadstoffe erlauben. Aber auch die Fragen der Besiedlung und Bebauung einschl. der Standortbestimmung von Industrieanlagen bedürfen sorgfältiger Untersuchungen und Planungen. Neben den regelmäßigen Messungen gehören hierzu auch die Ermittlung der Emissionen durch Erstellen von Emissionskatastern, die Fortentwicklung von Berechnungen uber die Ausbreitung von Luftschadstoffen in der Atmosphäre, die bioklimatischen Untersuchungen sowie epidemiologische Erhebungen über den Einfluß der Luftverunreinigungen auf die Gesundheit des Menschen. Die Erarbeitung von Luftreinhalteplänen muß fortgesetzt bzw. vorhandene Pläne dieser Art müssen weiterentwickelt und fortgeschrieben werden.

12) Kuhl (1977), S. 45 . 
räumlich und fachlich aus. 13)

(6) Eine umfassende Bewertung der bundesrepublikanischen Raumordnungsplanung und -politik unter umweltpolitischen Effizienzgesichtspunkten hat daher nicht nur die raumbezogenen Aktivitäten auf Bundes- und Landesebene, sondern auch unbedingt raumwirksame Maßnahmen von Trägern der Regionalplanung zu berücksichtigen. 14 )

Die an das ROG gebundene Regionalplanung hat innerhalb des von den Landesentwicklungsplänen gesetzten Rahmen zu erfolgen, wobei die in ihnen ausgewiesenen zentralen orte und Entwicklungsachsen auch von erheblicher Umweltrelevanz sind. Denn mit dem zentralörtlichen System - einem System der dezentralen geordneten Verdichtung von Wohn- und Arbeitsstätten in geeigneten Gemeinden, mit dem man interregional annähernd gleicheinfra- und wirtschaftsstrukturelle Bedingungen bei gleichzeitiger Sicherung von ökologischen Ausgleichsräumen in der Nähe der jeweiligen Zentren erreichen will - und der Bestimmung von Entwicklungsachsen ( $\rightarrow \mathrm{Zu-}$ sammenfassung der verkehrs- und kommunikationsbezogenen Bandinfrastruktur) können geeignete instrumentelle Voraussetzungen für eine zersiedelungshemmende und freiraumerhaltende (bzw.- erweiternde) Kontrolle der großräumigen Raumnutzung etabliert sein. Die Regionalplanung mit ihrer Aufgabe, im Vergleich zur gesamt-

13) Mit diesen Feststellungen soll nicht suggeriert werden, daß hier einer Konzeption allumfassender Raumplanung mit bindenden Detailregelungen das Wort geredet wird. Weiter unten $(\rightarrow$ Textziffer 7 ) wird auf die Gefahren von juristischen Normengefügen für die Allokationseffizienz hingewiesen; denn solche Normensysteme haben einen statischen Charakter, der der Dynamik des Wirtschaftsprozesses nicht gerecht wird und suboptimale Entwicklungen fördert.

14) In dieser Studie interessieren vorwiegend großräumige, überörtliche Probleme, so daß die (örtliche) Bauleitplanung - die den Gemeinden bzw. Gemeindeverbänden unterliegt - hier von der weiteren Betrachtung ausgeschlossen bleibt. Lediglich bei den untenstehenden Ausführungen zur TA Luft wird mittelbar ein Zusammenhang mit Problemen der Bauleitplanung hergestellt; denn diese hat sich an den Werten der TA Luft zu orientieren. - $\mathrm{Zu}$ den besonderen Problemen der kommunalen Umweltpolitik vgl. z.B. IfU (1978). 
flächendeckenden Landesplanung auch Details vollständig und gründlich aufzuarbeiten, beeinflußt die praktische Umsetzung des Konzepts der zentralen Orte und Entwicklungsachsen in einem ebenso hohen Maße wie die Realisierung des Konzepts der Vorrangebiete. 15)

Beide Konzepte resultieren aus dem Gedanken der räumlich-funktionalen Arbeitsteilung, nach dem die einzelnen Teile eines Gesamtgebietes ihre jeweiligen komparativen Vorteile nützen (z.B. geographische Lage, Ausstattung mit natürlichen Ressourcen, (mittelfristig invariables) Besiedlungsprofil von Landschaften etc.) und in Tauschbeziehungen zueinander treten, was einen prosperitätszuwachs in diesem arbeitsteilig organisierten und von Mobilitätsschranken befreiten Raum begünstigen soll.

Der Regionalplanung mit ihrer Detaillierungsaufgabe ${ }^{16)}$ kommt insbesondere auch eine luftreinhaltepolitische Bedeutung zu, etwa durch die Ausweisung eines Industrieansiedlungsbereichs in der Nähe eines Wohngebietes. Die regionalen Planungsträger sind jedoch aufgrund vager Rechtsbegriffe ${ }^{17)} \mathrm{zu}$ wenig an Umweltschutzbelange gebunden, wodurch rein vollzugstechnisch irreversible Umweltschäden vor Einsetzen einer Landesplanungskontrolle möglich erscheinen. Nach $\S 50$ BImSchG obliegt zwar sämtlichen Planungsträgern eine generelle Koordinationspflicht, aber der Entschei-

15) In Anlehnung an die vorhandene Literatur werden hier unter Vorranggebieten durchweg Freiräume verstanden, in denen spezielle Funktionen (z.B. Freizeit und Erholung, Sicherung der Wasserversorgung, besondere ökologische Ausgleichsfunktionen, Land- und Forstwirtschaft, Rohstoffgewinnung) gegenüber den in sog. Verdichtungsräumen vorherrschenden Raumnutzungen ( $\mathrm{z} . \mathrm{B}$. industrielle bzw. gewerbliche produktion, dichte Wohnbesiedelung und Verkehrsinfrastruktur) dominieren.

16) So haben beispielsweise die nordrhein-westfälischen Regionalpläne - letztendlich auch umweltschutzrelevante - Angaben über "die angestrebte Struktur des Planungsgebietes, insbesondere im Hinblick auf Siedlung, Verkehr, Wirtschaft, Landwirtschaft, Ver- und Entsorgung, Umweltschutz, Landschaftsordnung, Erholung und Bildung... sowie über "die angestrebte durchschnittliche Siedlungsdichte in den Wohnsiedlungsbereichen" zu machen. Zitiert nach Kuhl, G. (1977), S. 93.

17) Zur detaillierten juristischen Begründung vgl. Kuhl, G. (1977), S. 104-107. 
dungsspielraum im Kontext mit umweltqualitätstangierenden Planungsvorhaben ist nicht essentiell eingeengt; denn sowohl nach dem Abwägungsgebot gemäB $\S 2$ Abs. 2 ROG als auch nach der in $\S 50$ BImSchG enthaltenen Formulierung, nach der bei raumbedeutsamen Maßnahmen Wohn- und sonstige Schutzgebiete "so weit wie möglich" von Umweltbelastungen auszunehmen sind, wird dem Umweltschutz kein a-priori-Vorrecht eingeräumt. ${ }^{18)}$

(7) Der in diesem Abschnitt skizzierte überblick über die Einbettung von Umweltschutzanliegen in raumordnungsrelevante Gesetzeswerke und Programme bzw. Pläne zeigt u.a., daß der von den geltenden Normen ausgehende Lenkungseffekt für den umweltpolitischen Allokationsprozeß zu einem wesentlichen Teil von den Vollzugsmöglichkeiten und -praktiken untergeordenter Gebietskörperschaften und Behörden abhängt. 19)

Um verbesserte Voraussetzungen für aufeinander abgestimmte Regional- und Umweltpolitiken zu schaffen, ist zunächst eine grundlegende Entscheidung $z u$ fällen: in welchem Umfang soll eine Veränderung bzw. eine Verdichtung des bestehenden raumordnungsrelevanten Normengefüges angestrebt werden, um sowohl den bei den unteren Gebietskörperschaften vorhandenen Entscheidungsspielraum im Sinne einer generellen Umweltbindung einzuengen als auch auf horizontalen Ebenen die Koordination zwischen den einzelnen Ressorts entsprechend zu verbessern?

Umweltstandards für die Raumordnungsplanung könnten z.B. wie folgt beschaffen sein: 20 )

18) Auch die Luftreinhaltepläne gemäß $\S 47$ BImSchG sind für die Raumordnung nur von begrenzter Bedeutung, da sie sich lediglich auf Belastungsgebiete beziehen ( $\rightarrow$ Abschnitt 4 ).

19) Vgl. zur Vollzugsproblematik insbesondere die Ausführungen in Mayntz et.al. (1978).

20) Kuhl (1977), S. 120 ff. - Die SPD-Thesen zur Umweltpolitik von 1975 zeigen eine Präferenz zugunsten eines Umweltstandardsystems im Rahmen der Raumordnung an. Vgl. auch Bullinger (1974). 
(i) Standards für die Aufteilung der Gesamtfläche auf die einzelnen Nutzungsformen, 21)

(ii) Flächenimmissionsstandards, 22)

(iii) Standards für Gewässerbelastungen,

(iv) Standards für regionale Infrastrukturausstattungen mit unmittelbaren Umwelteffekten (z.B. Verkehrswegenetz, Verund Entsorgungsanlagen).

Eine solche normenorientierte Strategie zur Etablierung von Umweltschutzbelangen im Raumplanungsprozeß 1 äßt jedoch einen zeitintensiven politischen Entscheidungsfindungsprozeß erwarten, möglicherweise unter der Beteiligung von Gerichten. Aber nicht nur die Höhe der vielschichtigen Implementierungskosten, deren Ansteigen mit zunehmendem Reglementierungsgrund des regionalund umweltpolitischen Instrumentariums zu erwarten ist, 23) sondern auch wirtschaftsordnungspolitische Aspekte lassen Skepsis gegenüber einem Normierungsansatz als angebracht erscheinen. Denn die sich im föderativen System der Bundesrepublik von übergeordneter $\mathrm{zu}$ untergeordneter stufe vollziehende Konkretisierung raumordnungspolitischer Vorgaben kann durch zusätzliche, umweltpolitisch motivierte Auflagen zu einem systemsprengenden Detaillierungsgrad heranreifen. Gesetzeswerke und Pläne zur Raumordnung stellen dann im Extremfall nicht mehr Rahmenbedingungen für das Marktgeschehen dar, sondern entwickeln sich zu Marktprozesse aufhebenden Totalbedingungen. Jeder umweltindizierte, auf eine Perfektionierung der raumordnungsrelevanten Vorschriften zielende Vorschlag ist also auf seine Marktkonformität zu überprüfen. Nach Regionen ausgewiesene Zuzugsverbote, Ansiedlungsgebote oder

21) Der Arbeitskreis 5 des Beirats für Raumordnung erstellte 1976 eine Liste gesellschaftlicher (incl. umweltbezogener) Indikatoren, die evtl. als Schlüssel für solche Flächennutzungszuteilungen dienen könnten.

22) Unten ( $\rightarrow$ Abschnitt 4 ) wird die Problematik einer starren Immissionsnormierungspolitik anhand des BImSchG diskutiert.

23) Zur Stützung dieser These vgl. z.B. auch Fishelson, G. (1979, S.171), Siebert (1979, S. $9 \mathrm{f} ; 1976, \mathrm{~s} .71 \mathrm{ff})$. 
Outputlimitierungen von Betrieben können allenfalls unter besonderen Bedingungen, wie etwa gesundheitsgefährdende Immissionssituationen als temporär notwendig erachtet werden. 24)

Eine - mit dem Ziel einer angemessenen Erfassung von Umwelteffekten durchgeführte - Reform der raumwirksamen Planung in der Bundesrepublik hat das geltende umweltpolitische Instrumentarium zu berücksichtigen. Insbesondere die luftreinhaltepolitisch bedeutsamen Immissionsschutzregelungen ( $\rightarrow$ BImSchG) ermöglichen Maßnahmen von erheblicher regionalpolitischer Tragweite. In Abschnitt 4 wird ausgeführt, wie eine unbewegliche, pauschal agierende Umweltstandardpolitik auf Grundlage des BImSchG über eine reduzierte Faktormobilität zur Verkrustung von regionalen Wirtschaftsstrukturen beitragen kann und damit die langfristige Wettbewerbsfähigkeit einer Region mindert. Vorgeschlagene Änderungen des BImSchG, die auf ein Mehr an marktwirtschaftlicher Flexibilität und auf ein Weniger an strukturfestschreibenden Normen hinauslaufen, 25) haben daher von seiten der Raumordnung Flankenschutz $\mathrm{zu}$ erhalten. Somit verspricht eine isoliert vorgenommene, umweltschutzorientierte Normenverdichtung innerhalb der raumordnungsgesetzlichen Regelungen ${ }^{26)}$ keine effiziente ${ }^{27}$ ) Lösung des umweltund regionalpolitischen Koordinationsproblems.

24) Die Smogpläne in der Bundesrepublik sind diesem Gedanken zuzuordnen.

25) Das BMI hat z.B. wiederholt darauf hingewiesen, daß in der augenblicklichen "post-legislativen" Phase der Umweltpolitik vermehrt Instrumente marktwirtschaftlichen Charakters gegenüber Auflageninstrumenten eingesetzt werden sollten.

26) Kuhl (1977) und Bullinger (1974) tendieren zu einem solchen, einseitigen Lösungsansatz.

27) "Effizienz" ist hier aufzufassen im Sinne der Minimierung von gesamtwirtschaftlichen (Opportunitäts-) Kosten bei dem Übergang von einer (unerwünschten) Ausgangssituation zu einer Wunschsituation. 
3. Zur umweltpolitischen Relevanz der praktischen Regionalpolitik am Beispiel des regionalen Fördersystems in der Bundesrepublik

(8) Die Intention der 1969 per Gesetz beschlossenen Bund/LänderGemeinschaftsaufgabe "Verbesserung der regionalen Wirtschaftsstruktur"28) ist es, wirtschaftliche Aktivitäten in Gebieten zu fördern, deren Wirtschaftskraft merklich unter dem Bundesdurchschnitt liegt (bzw. eine entsprechende Entwicklungstendenz aufweist) und "in denen Wirtschaftszweige vorherrschen, die vom Strukturwandel in einer Weise betroffen sind und bedroht sind, daß negative Rückwirkungen auf das Gebiet in erheblichem Umfang eingetreten oder absehbar sind" [Deutscher Bundestag (1971), 1 , Abs. 2 ]. Das damit gegebene regionalpolitische Instrumentarium zielt eindeutig auf Regionen mit anhaltenden Beschäftigungsproblemen $\mathrm{ab}$ und läßt umweltpolitische Ziele unberücksichtigt. So wählt z.B. der Planungsausschuß ${ }^{29)}$ der Gemeinschaftsaufgabe die Fördergebiete mit Hilfe eines Indikators aus, in denen Kennziffern über Einkommenserzielungsmöglichkeiten, die aktuelle und erwartete Arbeitsmarktsituation und die Infrastrukturausstattung eingehen. ${ }^{30}$ )

(9) In Anbetracht einer solchen Konzeption, die ausschlieblich auf die Nivellierung der regional unterschiedlich ausgeprägten Wirtschaftskraft abzielt, verwundert es z.B. nicht, daß bei industriellen Neuansiedlungsprojekten im Rahmen der Wirtschafts-

28) Deutscher Bundestag (1971).

29) In diesem - mit Koordinationsaufgaben bezüglich der einzelnen regionalen Aktionsprogramme versehenen - Gremium verfügen Bund und Länder (entsprechend ihren Förderungskostenbeteiligungen) jeweils über die Hälfte der Stimmen, ein einzelnes Bundesland ist also in jedem Fall überstimmbar, weshalb auch mitunter von einem zentralistischen $\mathrm{zug}$ der regionalen Wirtschaftsförderung gesprochen wird, der einen "Fremdkörper" innerhalb des bundesrepublikanischen Föderalismus darstellt. Vgl. z.B. Fürst/Klemmer/Zimmermann (1976, S.142).

3o) Saar- und Zonenrandgebiet werden von vornherein als Fördergebiete eingestuft. 
förderung die Umweltschutzbehörden bislang nur unzureichend in den Planungsprozeß miteinbezogen sind (Umweltgutachten 1978, S. 378).

Daß Aspekte des Umweltschutzes bei der Ausgestaltung des zentralen regionalpolitischen Instrumentariums der Wirtschftsförderung vernachlässigt wurden, konnte damit gerechtfertigt werden, daß bereits mit den bestehenden gesetzlichen oder gesetzesähnlichen Regelungen 31) ausreichende Umweltschutzrestriktionen vorliegen. Wie jedoch die Ausführungen im vorausgehenden Abschnitt gezeigt haben, ist die faktische Durchsetzung von Umweltschutzbelangen wegen vager Rechtsbegriffe und/oder relativ weiter Entscheidungsspielräume der beteiligten Behörden auf allen Ebenen des föderativen Systems nur ungenügend gewährleistet. Von daher wäre es angebracht gewesen, auch ein auf regionale Beschäftigungsprobleme ausgerichtetes Instrument mit einem Umweltvermerk $z u$ versehen; denn die bisherigen Erfahrungen deuten darauf hin, daß die umweltbezogenen Richtlinien der Raumordnungsplanung nur dann umweltpolitisch nennenswert wirksam zu werden versprechen, wenn auf der Ebene des regionalpolitischen Instrumenteneinsatzes auch Umwelteffekte in Rechnung gestellt werden ${ }^{32}$ ).

(10) Der fehlende umweltpolitische Bezug der Gemeinschaftsaufgabe mag auch damit zusammenhängen, daß die Beziehungen zwischen Wirtschaftswachstums - und Umweltschutzzielen oft als konfliktträchtig eingeschätzt wird.

31) Gemeint sind damit u.a. das ROG, die Landesplanungsgesetze, das Bundesraumordnungsprogramm, die Landes- oder Regionalentwicklungspläne oder auch die Entschließung "Raumordnung und Umweltschutz" der Ministerkonferenz für Raumordnung 1972.

32) Denkbar sind beispielsweise Umweltverträglichkeitsprüfungen für Industrieansiedlungsprojekte in einer beschäftigungspolitischen Problemregion, um innerhalb dieses Gebietes zwischen umweltpolitisch erwünschten und unerwünschten standorten differenzieren $\mathrm{zu}$ können. Solche Untersuchungen können von Regionalbehörden veranlaßt werden, welche die Ergebnisse dann über das betreffende Bundesland in den Planungsausschuß der Gemeinschaftsaufgabe einbringen können. 
Mehrere umfangreiche Untersuchungen führten jedoch in Widerlegung dieser These zu dem Resultat, daß die Umweltpolitik in der Bundesrepublik zu einem gesamtwirtschaftlich positiven Nettozuwachs an Arbeitsplätzen führt: nach Sprenger (1979) hat die Umweltpolitik zu einer Verstetigung der Nachfrageentwicklung sowie zu einer verbesserten Kapazitätsauslastung und einer stabilisierten Beschäftigungssituation beigetragen; ${ }^{33)}$ auch das Rheinisch-Westfälische Institut für Wirtschaftsforschung Essen weist in seinem Strukturbericht (1980) für die Bundesregierung auf die relativ niedrigen effektiven Zusatzkosten und neue investive Perspektiven für Unternehmen hin, die von urnweltpolitischen Aktivitäten betroffen sind. 34 )

Die Frage, die in dem hier zu behandelnden regionalpolitischen Kontext besonders interessiert, bezieht sich auf die regional- und sektorstrukturelle Beschaffenheit von umweltschutzinduzierten Wachstumsimpulsen. Und zwar steht zur Debatte, ob und wie die Strategie der regionalen Wirtschaftsförderung sich die Dynamik eines neuen und sich im Aufbaustadium befindlichen Wirtschaftszweiges - nämlich die Umweltschutzindustrie - zunutze machen kann, indem ökonomisch relativ unterentwickelte Regionen bevorzugt an dieser speziellen Expansion partizipieren.

Die aktuelle Situation ist gekennzeichnet von einer räumlichen Konzentration der Betriebe mit einer auch für den Umweltschutz relevanten produktpalette in Belastungsgebieten. ${ }^{35}$ ) Es liegt nahe, diese standortcharakteristika auf regional gebundene Fühlungsvorteile wie Problemnähe, Nachfragekonzentration und Verkehrsinfrastruktur zurückzuführen. Das kann auch dadurch als bestätigt gel-

33) Meißner/Hödl (1976) kommen zu ähnlichen Ergebnissen; so wurden für das Jahr 1975200.000 umweltschutzinduzierte Arbeitsplätze errechnet, und 250.000 zusätzlich Beschäftigte für 1980. Vgl. BMI-Umwelt 71 (1979, S. 3). Vgl. weiterhin dazu Meißner $(1979,1980)$, Kabelitz (1980) sowie Ullmann/zimmermann (1980).

34) Vgl. auch Ullmann/Zimmermann (1980).- In den USA wurden vom Council on Environmental Quality (CEQ) ebenfalls positive Beschäftigungswi rkungen der dortigen Umweltschutzgesetzgebung festgestellt; vgl. z.B. CEQ (1975; S. $494 \mathrm{ff})$.

35) Gemeint sind damit Gebiete, die einer relativ starken Belastung durch Luftschadstoffe unterliegen. Vgl. Knoepfel/Weidner (1980, S. 170). 
ten, daß oft gerade emissionsintensiv produzierende Betriebe angesichts umweltpolitisch gesetzter Restriktionen - umweltschutztechnologische Innovationen entwickeln und sich dadurch neue (Investitionsgüter-) Märkte erschließen. ${ }^{36}$ )

Für die Bundesrepublik ist jedoch festzuhalten, daß die Fördergebiete in der Regel nicht mit relativ stark luftverschmutzten Zonen übereinstimmen. ${ }^{37)}$ Das bedeutet aber, daß eine dem Ziel der Gemeinschaftsaufgabe - nämlich regionale Wirtschaftskraftdisparitäten weitestgehend abzubauen - entgegengerichtete Entwicklung einsetzt. Am Beispiel der Umweltschutztechnologie zeigt sich, daß im allgemeinen die Entwicklung neuer Technologien und Produkte eher regional konzentriert in Ballungsräumen als regional gleichmäßig verteilt erfolgt und daß auch die neuen produktionstechniken zunächst nur an wenigen standorten angewendet und die neuen Erzeugnisse lediglich in relativ eng dimensionierten Räumen hergestellt werden (Siebert, H., 1967).

(11) Es wäre eine als illusionär einzuschätzende strategie der regionalen Wirtschaftsförderung, wollte man eine regionale Gleichverteilung von Erstinnovatoren anstreben. Die Regionalpolitik hat stattdessen auf eine regionale Förderung der Diffusion von technologischen Neuerungen abzuzielen (BMBau (1980), S. 12). Instrumentell impliziert das im wesentlichen eine Abkehr von globalen finanziellen Investitionsanreizen und eine Hinwendung $z u$ selektiven finanziellen Anreizen für zukunftsträchtige Investitionen sowie die Etablierung von Beratungsinstitutionen (z.B. extern angebotene managementorientierte Serviceleistungen).

Dieses Konzept einer innovationsorientierten Regionalpolitik ${ }^{38}$ ) versucht auf eine langfristige interregionale und internationale

36) Knoepfel/Weidner (1980, I), S. 59; Ullmann/Zimmermann (1980).

37) Ausnahme: das Steinkohlegebiet Saar.

38) Vgl. dazu auch Adlung/Thoroe (1980), BM Bau (1980). 
Wettbewerbsfähigkeit von wirtschaftlich relativ rückständigen Gebieten hinzuwirken und basiert auf der Kritik an dem bisher praktizierten Vollzug der Gemeinschaftsaufgabe. ${ }^{39}$ ) Vor allem die Einwände, welche die Durchführung der regionalen Wirtschaftsförderung betreffen, sindhierwegen ihrer potentiellen umweltpolitischen Relevanz von Interesse. ${ }^{40)}$

Nach dem Auftrag des Gemeinschaftsaufgabengesetzes hat die Regionalförderung auch die vom strukturwandel evtl. ausgehenden wirtschaftskraftschwächenden Auswirkungen zu antizipieren und entsprechende Gegenmaßnahmen einzuleiten. Diesem längerfristigen, entwicklungspolitischen Aspekt, nach dem eine erhöhte und zukunftsträchtige Wettbewerbsposition für die förderungswürdigen Regionen durch eine geeignete Mittelvergabe zu sichern ist, wird aber die gegenwärtige praxis nicht gerecht. Die praktizierte Förderung ordnet dem konkurrenzbezogenen Zukunftskriterium kein besonderes Gewicht zu. Als Folge davon droht eine perpetuierung der Strukturschwäche von Förderregionen; denn industrielle Ansiedlungsprojekte und vorhandener Industriebesatz in diesen Regionen sind unterdurchschnittlich humankapitalintensiv ${ }^{4}$ ) und gekennzeichnet von einer produktionsstruktur mit einem 'bias' zugunsten

39) Die Gemeinschaftsaufgabe umfaßt im einzelnen die Förderung des Infrastrukturausbaus durch Zuschüsse für Projekte der wirtschaftsnahen Infrastruktur und - als zweites Hauptelement die finanzielle Unterstützung der privaten Investitionstätigkeit durch Investitionsdarlehen - und - zuschüsse ( $\rightarrow$ regionale Wirtschaftsförderung) .

40) Der Infrastrukturförderung wird mangelnde Großräumigkeit vorgeworfen: anstelle von Projekten mit dominant lokalem Charakter sollten bevorzugt Vorhaben unterstützt werden, die eine weite räumliche Ausstrahlung versprechen. Beispielsweise könnte eine verkehrsinfrastrukturelle Erweiterung (z.B. Ausbau des Schnellstraßensystems oder Herstellung von IC-Anschlüssen) den Innovationsfluß von den mit Agglomerationsvorteilen ausgestatteten Zentren in die Randzonen durch verbesserte Kommunikations- und Transportmöglichkeiten wesentlich erleichtern (vgl. Adlung/Thoroe (1980)).

41) Die Humankapitalintensität einer Branche könnte z.B. definiert werden als der Differenzbetrag zwischen der tatsächlichen Arbeitskostensumme und einem fiktiven Betrag, der sich im Falle der Eingruppierung aller Beschäftigten in die niedrigste Lohnstufe $(\rightarrow z . B$. Hilfsarbeiterlohn $)$ ergibt. Vgl.Adlung/Thoroe (1980), S. 7 . 
von standardisierten Produkten, deren Fertigung weniger gut ausgebildete Arbeitskräfte erfordert und die sich im Spätstadium ihres Reifezyklus befinden. Ausgehend davon, daß Klein- und Mittelunternehmen in den Problemregionen in der Regel nicht grundlegend neue Technologien entwickeln (und auch nicht zu deren Erstanwendern gehören), werden durch die undifferenzierte Förderungspraxis die Chancen strukturschwacher Zonen nicht verbessert, an den technologischen Stand und an das Einkommensniveau der Zentren AnschluB finden zu können und somit langfristig einen emanzipierten Status im Rahmen des gesamtregionalen arbeitsteiligen Prozesses einnehmen zu können. ${ }^{42)}$ 43)

(12) Welche umweltpolitischen Implikationen mit der geltenden Regionalförderung im Unterschied zu dem Reformkonzept einer innovationsorientierten Regionalpolitik verbunden sind, kann an dieser stelle nur vorbehaltlich empirischer tberprüfungen erörtert werden. Denn bislang fehlen umfassende empirische Untersuchungen zu den Zusammenhängen zwischen Humankapitalintensität und der Emissionsneigung einzelner Industriezweige. ${ }^{44)}$ Als eine erste Arbeitshypothese bietet es sich an, relativ humankapitalschwachen Betrieben mit hoher Sachkapitalausstattung und einem großen Anteil

42) Darüber hinaus geht von den Entwicklungsländern ein wachsender Konkurrenzdruck auf den Weltmärkten standardisierter Produkte aus, so dab auch von daher eine vermehrt innovationsorientierte Regionalpolitik in der Bundesrepbulik not tut.

43) Als ein weiterer Punkt der Kritik an der Regionalförderung wird angefuhrt, da $\beta$ auch Rationalisierungsinvestitionen unterstützt werden, von denen letztlich eine arbeitsplatzsparende Wirkung ausgeht. Betrachtet man Rationalisierungsmaßnahmen als eine innovationspolitische Notwendigkeit, wird der Konflikt zwischen einer kurzfristig konzipierten Beschäftigungspolitik und einer längerfristig orientierten Arbeitsmarktpolitik offenbar.

44) Sprenger/Britschkat (1980) untersuchen empirisch die Sektorstruktureffekte der Umweltpolitik. - Ullmann/Zimmermann (1980) befassen sich in einer empirischen studie mit der regional- und strukturpolitischen Bedeutung der Umweltschutzindustrie. 
von "relative unskilled labour force" eine größere Emissionsneigung zuzurechnen als verhältnismäßig humankapitalintensiv produzierenden Unternehmen. Eine revidierte Regionalpolitik in der Bundesrepublik im Sinne eines forcierten Technologietransfers in wirtschaftliche Randzonen würde demnach einen geringeren zusätzlichen umweltschädigenden Effekt im Zuge der Industrieansiedlung in bisher schwach industrialisierten Gebieten mit vergleichsweise niedrigeren Immissionsniveaus bedeuten. Eine solche innovationsorientierte Regionalpolitik würde unterstützende Rahmenbedingungen für eine regionale Umweltpolitik mit sich bringen, die ökonomisch wenig erschlossene Wirtschaftsräume hoher Umweltqualität nicht als "ökologische Auffüllbecken" betrachtet, also nicht die hohen Immissionswerte von Verdichtungsräumen als Norm für die Gesamtregion setzt. ${ }^{45)}$

Es bleibt weiterhin zu prüfen, ob und wie regional differenzierte Immissionshöchstwerte (zusammen mit standardisierten Emissionswerten für bestimmte Anlagetypen) ${ }^{46)}$ zu einem gesamtregional etwa gleich knappen Angebot an schadstoffaufnehmenden und -assimilierenden Umweltdiensten führen können. Ausreichend höhere Umweltqualitätsanforderungen in relativ unbelasteten Räumen könnten nämlich - abgesehen von umweltpolitischen Absichten - regionalpolitische Nebeneffekte haben. Denn diese regionale Differenzierungsstrategie mag für die innovative Umweltschutzindustrie einen Anreiz darstellen, als standort nicht nur belastete wirtschaftszentren in Betracht zu ziehen, ${ }^{47}$ ) sondern auch strukturschwache Gebiete, in denen dann evtl. bereits ansässige Betriebe wegen restriktiv wirkender Immissionsschutzbestimmungen eine Nachfrage nach Umweltschutzinvestitionsgütern entwickeln oder ansiedlungswillige

45) An diesem Punkt setzt u.a. auch die Novellierungsdiskussion zum Bundesimmissionsschutzgesetz an. Vgl. Textziffer 19.

46) Siehe Abschnitt 4.

47) Siehe Textziffer 10. 
Unternehmen $^{48)}$ dieses Nachfragepotential erhöhen.

In der Palette der Bestimmungsfaktoren für die Standortwahl von Unternehmen ist der Faktor "Grad der umweltpolitischen Reglementierung" einer von vielen. Von daher sind der Beeinflussung der Standortentscheidung alleinig durch umweltpolitische Maßnahmen enge Grenzen gesetzt. ${ }^{49}$ ) Insbesondere die regional- und umweltpolitische Koordinierungsproblematik läßt sich nur durch ein geeignetes Instrumentenmix lösen. Sollen wirtschaftsstrukturschwache Gebiete mit unterdurchschnittlich hohen Immissionswerten ökonomisch vitalisiert werden ( $\rightarrow$ regionalpolitische zielsetzung) und dabei nicht an die wesentlich höheren Umweltbelastungen in Ballungszentren herangeführt werden $(\rightarrow$ umweltpolitische Zielsetzung), dann empfiehlt sich

(i) eine Abkehr von der bisher geübten praxis der eher pauschalen Investitionshilfe im Rahmen der regionalen Wirtschaftsförderung zugunsten eines selektiven Fördersystems, nach dem bevorzugt Investitionen mit Innovationscharakter in Problemregionen gelenkt werden sollen;

(ii) eine regionale Umweltpolitik, die durch entsprechende Vorgaben von regionalisierten Immissionshöchstwerten und anlagespezifischen Emissionswerten (gemäB TA Luft) strukturschwache Wirtschaftsgebiete mit hoher Umweltqualität nicht (wegen schwach- bzw. nichtbindender Umweltrestrik-

48) Es sei an dieser Stelle betont, daß nach diesem Konzept regional differenzierter Umweltqualitätsziele Unternehmen bei ihren Standortplanungen in Belastungs- und Freiräumen wegen unterschiedlicher Ausgangsimmissionen ähnlich restriktiv wirkende Umweltschutzbestimmungen vorfinden; Standortentscheidungen werden also primär von anderen Faktoren beeinflußt - z.B. von finanziellen Anreizen auf der Grundlage von regionalen Wirtschaftsförderungsprogrammen ( $\rightarrow$ Anmerkung 49).

49) Ausschließlich umweltpolitisch bedingte Verlagerungen von Produktionsstätten sind bislang in nicht nennenswertem Umfang erfolgt, wie z.B. I. Walter (1973) in einem internationalen Vergleich von Ländern mit unterschiedlich restriktiven Umweltpolitiken ermittelte. Vgl. auch ifo-schnelldienst 1-2/81,s.7-9. 
tion) zu andauernd attraktiven Standorten für emissionsintensive Industrien ${ }^{50)}$ werden läßt.

(13) Ein Maßnahmenbündel, das den Punkten (i) und (ii) gerecht wird, kann Industrialisierungsprozesse in Förderregionen verleiten, die sowohl zu einer langfristigen interregionalen und internationalen Konkurrenzfähigkeit der in diesen Gebieten erstellten Güter und Dienstleistungen führen als auch Chancen für das Fortbestehen wichtiger, vormals evtl. dominanter Umweltfunktionen (z.B. Erholung, Land- und Forstwirtschaft, ökologische Ausgleichsfunktion) eröffnen.

Für die Bundesrepublik trifft es $z u$, daß die Problemgebiete wegen der quantitativen und qualitativen Beschaffenheit ihres Arbeitskräftepotentials eher auf standardisierte Produkte fertigende Industrien attrahierend wirken als auf humankapitalintensive Unternehmen. Solche komparativen regionalen Ausstattungsvorteile können durchaus auch für eine zukunftsbezogene pragmatische Regionalpolitik von Bedeutung sein, indem diversifizierte Unternehmensansiedlungen angestrebt werden: gemeint ist damit etwa eine strukturpolitische Konzeption für Förderregionen, nach der Handelsverflechtungen innerhalb des Industriesektors ebenso berücksichtigt werden wie die Beziehungen zwischen Dienstleistungssektor und dem Bereich der industriellen Fertigung; die finanziellen Anreizsysteme sind demnach z.B. so auszugestalten, daß sich Betriebe traditioneller Industriezweige zusammen mit innovativen Unternehmungen - die eine zuliefererfunktion ausüben mögen (Beispiel: Umweltschutzindustrie) - in wirtschaftlich unterentwickelten Gebieten niederlassen. Einerseits wird dadurch eine wirtschaftliche Monostruktur vermieden, was der betreffenden Region zusätzlich Wachstumschancen eröffnet; andererseits besteht dadurch die Möglichkeit, daß von der regionalen Präsenz moderner

50) Ein kontrollierter, temporärer Immissionsanstieg in wirtschaftlich zu erschließenden problemregionen kann aber durchaus zulässig sein. 
Industrie- und/oder Dienstleistungsunternehmen Impulse zur technologischen weiterentwicklung ausgehen und sich auch innerhalb von bislang herkömmlich produzierenden Unternehmen innovative Produkt- und Verfahrensvariationen im Laufe der zeit durchsetzen. Ullmann/Zimmermann (1980) stützen in ihrer empirischen Studie diese These und stellen fest, daß die Umweltschutzindustrie mit ihrem hohen Anteil von vergleichsweise gut ausgebildeten Arbeitnehmern "unter dem Einfluß der Umweltschutzgesetze zu einem Mittelpunkt industrieller Innovation werden kann". ${ }^{51)}$

(14) Die Bedeutung des oben dargelegten Konzepts für die praktische Wirtschaftspolitik ist jedoch nicht zu überschätzen. Denn die Umweltschutzindustrie ist eine von vielen relativ humankapitalintensiven Wirtschaftszweigen und kann im Kontext einer innovationsorientierten Regionalförderung nur eine begrenzte Rolle spielen: nach Ullmann/Zimmermann (1980) sind nur 2,6 \& der Umweltschutzprodukte herstellenden Betriebe in als besonders strukturschwach zu klassifizierenden Räumen angesiedelt; auch für die nähere zukunft seien von der Umweltschutzindustrie regionalpolitisch keine wesentlichen Beiträge zu erwarten.52)53)

Für die Sanierung von Ballungsgebieten mit veralteten bzw. verstärktem internationalen Konkurrenzdruck ausgesetzten Industrien und mit hoher Umweltbelastung bietet sich die Förderung des Umweltschutzindustriesektors anstelle der Subventionierung von Betrieben mit eher traditionellen Produktionsprogrammen an. Eine Entlastung der Umwelt in Wirtschaftszentren ist als Folge einer solchen regionalen Strukturpolitik zu erwarten; außerdem eröff-

51) WZB-Mitteilungen Nr. 12 (1980), S. 8.

52) Weiterhin wird in dieser Studie auf die marginale Bedeutung der Umweltschutzindustrie zur Lösung der Arbeitsmarktproblematik hingewiesen.

53) Es sei hier ergänzend vermerkt, daß die Erreichung umweltpolitischer ziele durch den langfristigen Strukturwandel zugunsten des Dienstleistungssektors und zu Lasten von Primärund Sekundärsektor begünstigt werden kann. Präzise Feststellungen dazu bedürfen ausgiebiger empirischer Untersuchungen. Vgl. Sprenger/Britschkat (1980). 
nen sich dadurch neue Absatzchancen auf Inlands- und Auslandsmärkten mit expansiven Zukunftsperspektiven. 54)

4. Zur regionalen Umweltpolitik in der Bundesrepublik am Beispiel des Immissionsschutzes

(15) Dieser Abschnitt vermittelt zunächst einen tiberblick über die luftreinhaltepolitisch relevanten Immissionsschutzregelungen, wobei das Bundesimmissionsschutzgesetz im Vordergrund der Betrachtung steht. Weiterhin interessiert hier das Zusammenwirken der Gebietskörperschaften (vornehmlich Bund und Länder), die die entscheidenden Rahmenbedingungen für immissions -und raumwirksame Allokationsprozesse setzen. Abgestellt wird vor allen Dingen auf einige geltende Bestimmungen, deren inflexibler Charakter Tendenzen begünstigt, die einer effizienten Annäherung an umweltpolitische Zielvorgaben zuwiderlaufen und die interregionale Faktormobilität merklich einschränken. Es werden Vorschläge zur Vermeidung solcher Hemmnisse erarbeitet; dabei wird auf die idealtypische Emissionszertifikatelösung rekurriert.

(16) Der Beginn einer auf das Umweltmedium "Luft" ausgerichteten Umweltpolitik in der Bundesrepublik kann auf das Jahr 1959 datiert werden: damals wurde das "Gesetz zur Änderung der Gewerbeordnung und Ergänzung des Bürgerlichen Gesetzbuches" - auch als Immissionsschutzgesetz titulierbar - verabschiedet, das beträchtliche Reduzierungen der Gesamtstaubemissionen bewirkte (vgl. SRU (1978), S. 156).

Das umweltpolitische Instrumentarium wurde 1964 durch eine Verwaltungsvorschrift zur Gewerbeordnung - der "Technischen Anleitung zur Reinhaltung der Luft" (TA Luft) - erweitert. Die anlagentypbezogenen Emissionshöchstwerte, Produktstandards (z.B. für Brennstoffe) und Prozeßnormen, die durch diese Vorschrift vorgegeben werden, führten beispielsweise in den Jahren 1964 bis 1970

54) Vgl. auch Meyer-Bohm/Tokarski (1980). 
zu einem erheblichen Rückgang der Schwefeldioxyd-Immissionen. 55) 56)

Die TA-Luft wurde 1974 im Zusammenhang mit der Verabschiedung des Bundesimmissionsschutzgesetzes (BImSchG) revidiert, ${ }^{57}$ ) womit das Kernstück der - weitgehend noch heute verbindlichen - bundesdeutschen Luftgütepolitik etabliert war.

(17) Die Position, die das BImSchG inklusive dazugehöriger Rechtsverordnungen, Verwaltungsvorschriften und Richtlinien im föderativen System der Bundesrepublik einnimmt, basiert entscheidend auf einer Grundgesetzänderung von 1972, nach der die konkurrierende Gesetzgebungskompetenz des Bundes um das Gebiet der Luftreinhaltung erweitert wurde (Art. 74 Abs. 24 GG). Innerhalb des Systems bundeseinheitlicher Reglungen in Gestalt des BImSchG bleibt den Bundesländern lediglich eine das Bundesrecht konkretisierende Rechtsetzung. Demnach können die Länder Ermessensspielräume ausfüllen oder Ermächtigungen konkretisieren; es liegt hier also mit der Vollzugsautonomie der Länder eine Form des administrativen Föderalismus vor. Auch den Kommunen ist über eine "rechtsanwendende Rechtssetzung" ein Einfluß auf örtliche / überörtliche Immis-

55) In Ballungsgebieten war teilweise ein Rückgang um 70 ou verzeichnen, was auch auf der Verwendung schwefelärmerer Brennstoffe bei kleinfeuerungen und dem Bau höherer Schornsteine bei Großfeuerungen beruhte. Durch letzteres entstand jedoch innerhalb und außerhalb ( $\rightarrow$ Skandinavien) der Bundesrepublik eine Zunahme der säurehaltigen Niederschläge. Im übrigen gilt die Bundesrepublik als Netto-SO $\mathrm{S}_{2}$-Exporteur. (UBA (1977), S. 93). - Diese großräumige Emissionsverteilung läßt eine internationale Kooperation in Sachen Immissionsschutz als dringend geboten erscheinen.

56) Als ein weiteres Gesetz von luftreinhaltepolitischer Bedeutung ist das Benzin-Blei-Gesetz von $1971 \mathrm{zu}$ nennen: durch eine zeitlich abgestufte Verschärfung von Auflagen bezüglich des Benzinbleigehalts wurde ein deutlicher Rückgang der Bleibelastung erreicht. Siehe BMI-Umwelt 68 (1979), S. 22.

57) Die TA-Luft nimmt innerhalb der Immissionsschutzgesetzgebung die Stellung einer Ersten Allgemeinen Verwaltungsvorschrift des BImSchG ein. - Die Revision der TA-Luft von 1974 bestand im wesentlichen aus einer Anpassung an den "Stand der Technik". 
sionssituationen möglich. ${ }^{58)}$

(18) Die Natur des Umweltbereiches "Luft" bringt es mit sich, daß jede ihn betreffende Politik und gesetzliche Bestimmung mehr oder weniger von raumbedeutsamer Tragweite sind. Besonderes Augenmerk wird hier jedoch auf Komponenten des BImSchG gelegt, die einen unmittelbaren regionalpolitischen Bezug im Rahmen der Luftreinhaltepolitik aufweisen.

Folgende Elemente der in der Bundesrepublik geltenden Immissionsschutzregelungen sind daher in diesem Kontext von Interesse: ${ }^{59}$ )

(i) Anlagenbezogener Immissionsschutz: dabei ist zwischen genehmigungsbedürftigen Anlagen ${ }^{60}$ ) ( $\rightarrow$ Großemitten; siehe $\S \S 4 \mathrm{ff}$. BImSchG) und nicht genehmigungsbedürftigen Anlagen 61 )

58) Knoepfel/Weidner (1980), S. 135, 274).

59) Neben dem - hier näher erläuterten - anlagen- und gebietsbezogenen Immissionsschutz sind produktbezogene Regelungen von umweltpolitischer Bedeutung: z.B. Produktstandards für Brennund Treibstoffe bezüglich des Schwefelgehaltes in leichtem Heizöl und Dieselkraftstoff (3. BImSchGV vom 15.1.1975).

60) Bei den genehmigungsbedürftigen Anlagen ist wiederum zwischen einem förmlichen und einem vereinfachten Genehmigungsverfahren $z u$ unterscheiden. Letzteres schließt eine öffentliche Bekanntmachung des Vorhabens aus. In der 4. BImSchGV (vom 14 . $2.1975)$ ist für 100 Anlagearten festgelegt, welches Verfahren anzuwenden ist $(\rightarrow 60$ genehmigungsbedürftige, 40 nicht genehmigungsbedürftige Anlagetypen).

61) Die Luftreinhaltebelange dieser Anlagen werden innerhalb anderer Genehmigungsverfahren ( $\rightarrow$ baurechtliche Verfahren) geprüft, wobei dem Stand der Technik ( $\rightarrow$ Anmerkung 62) bzw. den technischen Anforderungen auf Basis von Rechtsverordnungen $z \dot{u}$ genügen ist. 
$(\rightarrow$ kleine Gewerbebetriebe, Hausfeuerungen) zu differenzieren. Die Genehmigung ist auf der Grundlage der TA Luft ${ }^{62)} z u$ erteilen, wenn die Anlage nicht umweltschädigend ${ }^{63)}$ wirkt bzw. durch Emissionsbegrenzungen - die dem Stand der Technik entsprechen ( $\S 5,6 \mathrm{BImSchG)} \mathrm{-} \mathrm{Vorsorge}$ gegen Umweltschäden getroffen wird. Die Betriebserlaubnis kann mit Auflagen erteilt werden, aber auch widerrufen oder nachträglich geändert werden, falls die ursprünglichen Genehmigungsbedingungen keinen ausreichenden Umweltschutz gewährleisten. Solche Änderungen dürfen aber nur dann veranlaßt werden, wenn sie wirtschaftlich vertretbar sind. 64) Die Inbetriebnahme einer Anlage hat ferner in Einklang mit bau-, planungs- und naturschutzrechtlichen Vorschriften des Bundes und der Länder zu stehen, um die Maßnahmen verschiedener Ressorts aufeinander abzu-

62) Die TA-Luft gibt für 10 Schadstoffe Kurzzeit- bzw. Langzeitimmissionshöchstwerte - bezogen auf die nähere Umgebung (i.d.R. 4 × $4 \mathrm{~km}^{2}$ ) von Anlagenstandorten - an. Außerdem enthält sie u.a. anlagenspezifische Emissionsgrenzwerte für 170 weitere Schadstoffe sowie eine Klassifikation von 40 Anlagetypen gemä $\beta$ des Standes der Technik, Produktstandards (siehe unten, TZ 22), Prozeßnormen (z.B. zeitliche Verteilung von Emissionen) und Meßvorschriften zur Immissionsermittlung. Als Stand der Technik gelten im Rahmen der TA Luft die DINNormen sowie Angaben des VDI - Handbuches "Reinhaltung der Luft", nach dem diese Daten von BMI veröffentlicht wurden (zur Problematik des Begriffes "Stand der Technik" vgl. z.B. auch SRU (1978), S.561 und die untenstehenden Ausführungen in Textziffer 22). - Die TA-Luft leistet für Verwaltungsbehörden durch technische Detailinformationen eine (rechtlich nicht zwingende) Konkretisierung unbestimmter Rechtsbegriffe. Die Bundesregierung strebt - ausgelöst durch den Fall Voerde (vgl. z.B. Siebert (1980)) - im Zuge der Novellierung des BImSchG an, die Immissionsgrenzwerte der TA Luft gleichermaßen verbindlich festzulegen und damit die geforderte Rechtssicherheit herzustellen ( $\rightarrow$ Vermutungsklausel).

63) "Nicht umweltschädigend" heißt hier, daß die Immissionsgrenzwerte gemäß TA Luft nicht überschritten werden ( $\rightarrow$ vorstehende Fußnote).

64) Vgl. dazu die kritischen Anmerkungen in Textziffer 22,Punkt (iv). 
stimmen.65)

(ii) Immissionsschutz für bestimmte Gebiete: Das Bundesimmissionsschutzgesetz ermöglicht auf der Basis von gesondert ausgewiesenen Regionen regional differenzierte Imissionsschutzmaßnahmen.

So werden bevorzugt Ballungszonen als Belastungsgebiete ausgewiesen, wozu die Landesregierungen befugt sind ( $\rightarrow \S \S 42 \mathrm{ff}$.BImSchG). Diese besonders verschmutzten Regionen unterliegen einer laufenden Luftqualitätskontrolle durch Landesbehörden: es werden Emissionskataster angelegt und fortwährende (mobile und stationäre) Immissionsmessungen ( $\rightarrow$ Immissionskataster) durchgeführt, deren Daten in Luftreinhaltepläne eingehen und dem dort enthaltenen Maßnahmenkatalog zur Verbesserung der Immissionssituation zugrundeliegen.

Nachstehende Abbildungen vermitteln einen Uberblick über die Struktur eines Luftreinhalteplanes und über den Zusammenhang zwischen Emissions- und Immissionskataster (aus: Rheinland-Pfalz/ Ministerium für Soziales, Gesundheit und Sport (1978), S. 21. f.).

65) Nach $\S 13$ BImSchG sind jedoch Ausnahmen von dieser Harmonisierungsvorschrift zugelassen. Inwiefern tatsächlich Abstimmungen stattfinden, kann nur durch eine Analyse der Vollzugspraxis ermittelt werden. - $\S 50$ BImSchG stellt einen Zusammenhang zwischen Umwelt- und Raumplanung her: demnach sind die Träger raumbedeutsamer Planungen verpflichtet, die Flächennutzungsplanung schonend im Hinblick auf Wohngebiete vorzunehmen. 


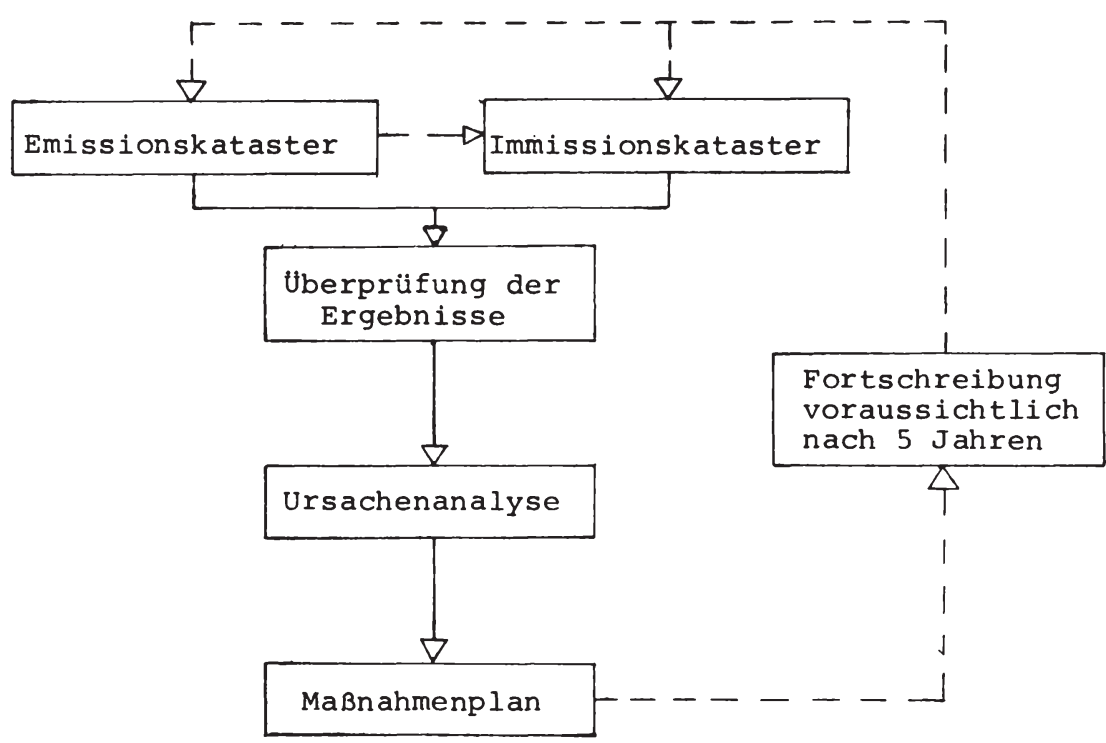

Schaubild 1: Die Struktur eines Luftreinhalteplanes

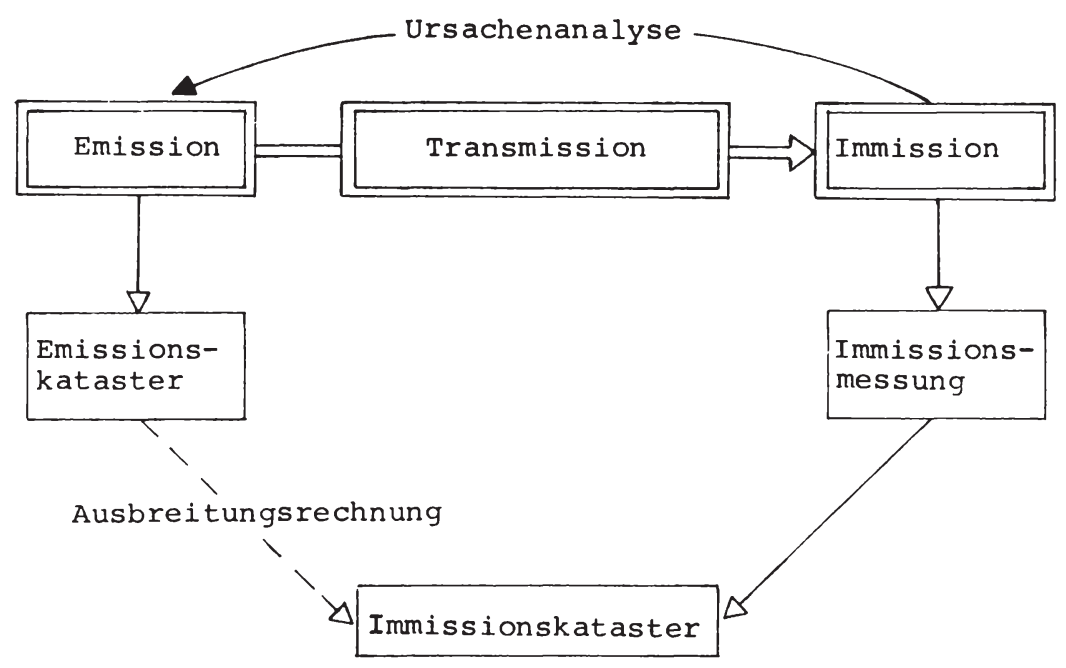

Schaubild 2: Der Zusammenhang zwischen Emissions- und Immissionskataster 
Als Beispiel sei der Luftreinhalteplan 1979-1984 für das Belastungsgebiet Ludwigshafen/Frankenthal genannt; darin werden Maßnahmen aufgeführt, die im industriellen und gewerblichen Sektor Anwendung finden können: ${ }^{66)}$

- Einhaltung des Standes der Technik zur Emissionsminderung

- Umstellung auf emissionsärmere Brenn- und Rohstoffe

- Anwendung emissionsärmerer Technologien bei gleichzeitiger Stillegung emissionsintensiver Anlagen

- In Einzelfällen Maßnahmen zur Abgasreinigung, die über den Stand der Technik hinausgehen

- Verbesserung der Ableitungsbedingungen für die nach dem Stand der Technik unvermeidbaren Emissionen.

Die übersicht über die zeitliche Entwicklung von ausgewählten industriellen Gesamtemissionen im Raum Ludwigshafen/Frankenthal belegt (zusammen mit dem übernommenen Kommentar), daß eine unerhebliche Verbesserung regionaler Umweltsituationen nur uber eine Umweltpolitik, die Anreize zur Weiterentwicklung der Umweltschutztechnologie bietet, erfolgen kann ( $\rightarrow$ Textziffer 23 ).

66) Rheinland-Pfalz/Ministerium für Soziales, Gesundheit und Umwelt (1980), S. 58. - Weitere Luftreinhaltepläne hat Nordrhein-Westfalen für die Belastungsgebiete Rheinschiene Süd (1977-1981), Ruhrgebiet West (1978-1982) und Ruhrgebiet Ost (1979-1983) vorgelegt. 


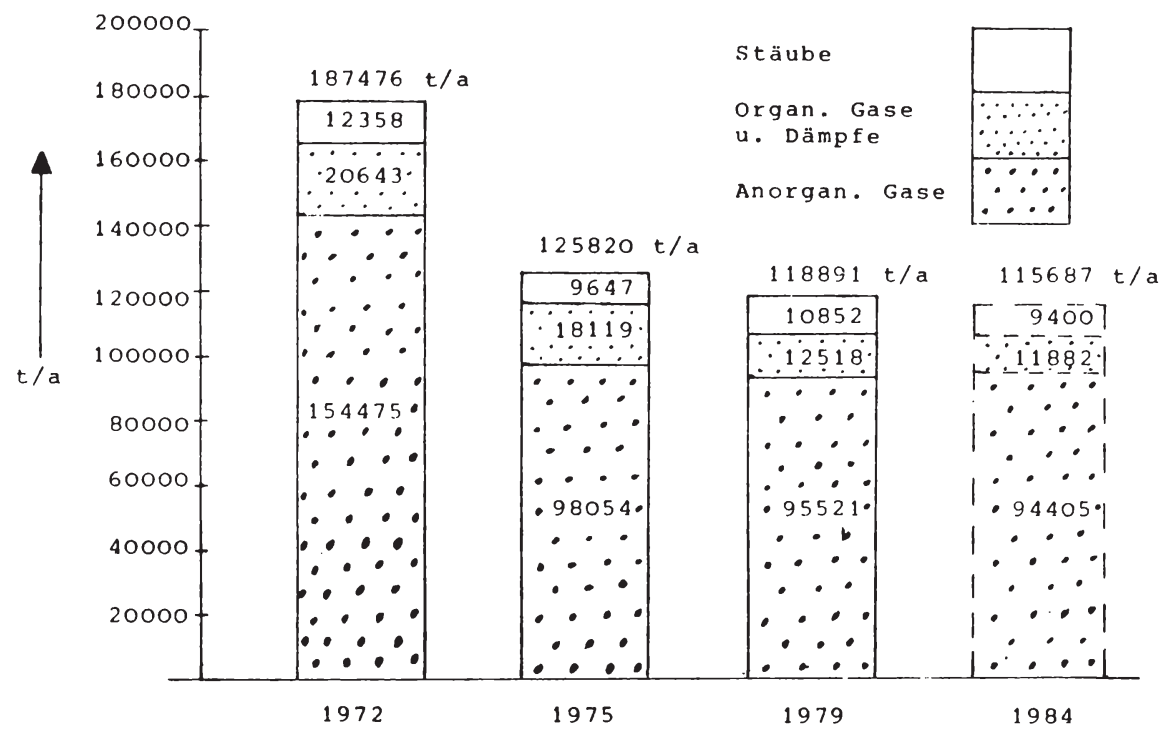

Schaubild 3: Entwicklung der Gesamtemissionen der Quellengruppe Industrie für Stäube, organische Gase und Dämpfe und anorganische Gase

Die Entwicklung der Gesamtemissionen im industriellen Bereich zeigt, daß bei einzelnen Quellen oder Anlagen durchaus noch beträchtliche Emissionsminderungen $z u$ erwarten sind, während die Reduzierung der Gesamtemissionen zunehmend an ihre Grenzen stößt, wo der Aufwand im Verhältnis zur erzielten Wirkung exponentiell steigt.

Diese Tendenz mußte sich zwangsläufig ergeben. Nachdem der Stand der Technik zur Emissionsminderung erreicht ist, lassen sich bei unverändertem Fortgang der Produktion weitere Emissionsverringerungen nur noch durch Fortentwicklung des Standes der Technik erzielen, die in der Regel mit erheblichem Aufwand verbunden ist. Aus: Luftreinhalteplan 1979-1984 Ludwigshafen/Frankenthal (Hrsg.: Rheinland/Pfalz/MSGU), S. 74 . 
Die Entwicklung der Gesamtemissionen im industriellen Bereich zeigt, daß bei einzelnen Quellen oder Anlagen durchaus noch beträchtliche Emissionsminderungen zu erwarten sind, während die Reduzierung der Gesamtemissionen zunehmend an ihre Grenzen stößt, wo der Aufwand im Verhältnis zur erzielten Wirkung exponentiell steigt.

Diese Tendenz mußte sich zwangsläufig ergeben. Nachdem der Stand der Technik zur Emissionsminderung erreicht ist, lassen sich bei unverändertem Fortgang der Produktion weitere Emissionsverringerungen nur noch durch Fortentwicklung des Standes der Technik erzielen, die in der Regel mit erheblichem Aufwand verbunden ist.

Die Kompetenzen der Länder erstrecken sich nicht nur auf die Bestimmung von Belastungsgebieten, 67)68) sondern auch auf die Festlegung von besonders schutzbedürftigen Gebieten $(\rightarrow \S 49$ Abs. 1 BImSchG) und Smoggebieten $\left(\rightarrow \S 49\right.$ Abs. 2 BImSchG $\left.{ }^{69}\right)$. - Für Schutzgebiete, die sowohl Schon- als auch Belastungsgebiete darstellen können, dürfen Produktionsverbote oder auch Errichtungsbzw. Betriebsbeschränkungen für Anlagen veranlaßt werden. Die Erfahrung zeigt, daß die Länder diese Reglementierung bislang noch nicht auf schongebiete angewendet haben.

67) Im Zusammenhang mit den jeweiligen Landesimmissionsschutzgesetzen wurden Rechtsverordnungen in Kraft gesetzt, die neben der Festlegung von Belastungsgebieten auch die Bestimmung von Smoggebieten regeln. Vgl. dzu die Übersicht bei Knoepfel/Weidner $(1980, \mathrm{~S} .138 \mathrm{ff})$.

68) Es bleibt zu ergänzen, daß Betreiber von genehmigungspflichtigen Anlagen, die sich in Belastungsgebieten befinden, auf jeden Fall zu Emissionserklärungen verpflichtet sind $(\rightarrow \S 27$ BImSchG); diese Erklärungspflicht ist jedoch nicht notwendigerweise auf Belastungsgebiete beschränkt) und einen Immissionsschutzbeauftragten einzusetzen haben $(\rightarrow \S \S 53 \mathrm{ff}$. BImSchG).

69) In Smoggebieten treten zur Uberwindung eines Immissionsnotstandes einschränkende Bestimmungen bezüglich Dauer und Intensität des Betriebes von emissionsträchtigen Anlagen, des Kfz-Verkehrs und z.B. des Schwefelgehaltes von Brennstoffen in Kraft. Auf Landesebene wurden durch Smogverordnungen die im einzelnen zu treffenden Maßnahmen näher festgelegt. 
(19) Die Möglichkeiten der Länder, umweltpolitisch Einfluß zu nehmen, sind auch dadurch von beträchtlichem Umfang, weil das BImSchG lediglich allgemeine Zielformulierungen enthält, also z.B. keine operablen Ziele in Form von bestimmten Emissions - und Immissionswerten vorgibt. In regionalen Luftreinhalteplänen werden jedoch konkrete Ziele ausgewiesen: z.B. werden im Plan 19791984 für die Region Ludwigshafen/Frankenthal Emissionsreduktionen nach dem Stand der Technik, die Immissionsminderung in übermäßig belasteten Gebieten ${ }^{70}$ ) und eine deutliche Unterschreitung von Immissionshöchstwerten in den verbleibenden Gebieten gefordert (Rheinland-Pfalz/MSGU (1980), S. 58).

In ihrem Erneuerungsentwurf zur Immissionsschutzgesetzgebung strebt die Bundesregierung eine präzisere Formulierung luftreinhaltepolitischer Ziele an. Vorgeschlagen werden - an dem Vorsorgegebot orientierte - Regelungen, nach denen prinzipiell für alle Regionen des Bundesgebietes Immissionsgrenzwerte zu gelten haben, die auch einen umfassenden Naturschutz sicherstellen, 71) wobei insbesondere Gebiete mit überwiegend land- und forstwirtschaftlicher Nutzung und/oder Erholungsfunktion zu berücksichtigen sind. Den Ländern soll es dabei einerseits möglich sein, in sog. Reinluftgebieten Ausnahmen vom Vorsorgegebot zuzulassen, falls an der Erschließung von Industriestandorten - z.B. aus beschäftigungspolitischen Gründen - ein dominierendes öffentliches Interesse besteht; andererseits könnten aber auch in Belastungsgebieten vorsorgestrategische Maßnahmen veranlaßt werden, um zumindest ein weiteres Absinken der Luftqualität $z u$ verhindern.

Der Bundesrat lehnt jedoch eine gesamtflächenabdeckende Regionalisierung von Immissionsgrenzwerten ab und will dagegen die Landesregierungen mit der Befugnis ausgestattet sehen, "Gebiete be-

70) Gemeint sind Gebiete, in denen die Immissionshöchstwerte bereits überschritten sind.

71) Nach dem Ergebnis einer Sachverständigenanhörung vom Februar 1978 in Berlin gewährleisten die menschliche Gesundheit schützende Schadstoffkonzentrationswerte nicht zwangsläufig den Schutz von Tieren, Pflanzen sowie bestimmten Sachgütern. 
sonderer Luftreinhaltung" zu bestimmen. Eine solche Lösung impliziert allerdings die Legalisierung eines bundesweit einheitlichen Immissionsniveaus, das einen auf die menschliche Gesundheit bezogenen, also relativ hohen Grenzwert darstellt ( $\rightarrow$ Anmerkung 16). 72) Die Bundesregierung plädiert im Gegensatz dazu für regional differenzierte Strategien, die unterschiedliche ökonomische und ökologische Gegebenheiten ausreichend würdigen. ${ }^{73)}$ Diese Politik einer Emissionskontrolle bei regional differenzierten Immissionszielen ist im übrigen eher mit den Resultaten regionaler Umweltallokationsmodelle zu vereinbaren. 74)

(20) Gebietsbezogene Immissionsnormen - wie z.B. in der TA Luft festgelegt - können zu einer regionalen Faktorimmobilität beitragen und somit suboptimale Faktorallokationen zementieren. ${ }^{75}$ ) Das kann beispielsweise bedeuten, daß umweltfreundichere produktionsanlagen deshalb nicht errichtet werden dürfen, weil in dem fraglichen Gebiet die Verschmutzung bereits die Höchstgrenze erreicht hat - obwohl vergleichbare Anlagen emissionsintensiver sind.

In ihrem BImSchG - Erneuerungsentwurf präsentiert die Bundesregierung Regelungen, die solche Schwierigkeiten bei der Genehmigung neuer Anlagen in den Zentren von Belastungsgebieten vermeiden sollen:

- gemäß Sanierungsklausel wäre ein Betreiber neuer Anlagen in Gebieten mit bereits überschrittenen Immissionshöchstwerten

72) So die Feststellung der Bundesregierung. Vgl. z.B. Umwelt (BMI) 69 (1979), S. 14 .

73) Zur BImSchG-Novellierungsdebatte vgl, auch BT-Drucksache (8/2751).

74) Vgl. Siebert, H. (1979), der ausführt, daß die umweltpolitische Allokationseffizienz einer ausreichenden Würdigung regionaler Diskrepanzen in Bezug auf Assimilationskapazitäten, Konsumentenpräferenzen, Bevölkerungsdichte sowie Industriebesatz bedarf.

75) Vgl. Bonus, H. (1980), Siebert, H. (1978a). 
verpflichtet, durch eigene Leistungen zu einer verringerten Luftbelastung beizutragen, und die von seinem alten und neu zu genehmigenden Anlagen ausgehenden Emissionen insgesamt um wenigstens 50 \& $\mathrm{zu}$ reduzieren; ${ }^{76)}$

- die Luftreinhalteplanklausel verlangt von der betreffenden Landesbehörde, im Rahmen eines Luftreinhalteplanes darauf hinzuwirken, daß in mit Luftschadstoffen überlasteten Gebieten diese ubberschreitungen unter Einschluß auch der Emissionen der neu zu genehmigenden Anlagen um mindestens ein Drittel innerhalb von fünf Jahren reduziert werden. ${ }^{77)}$

(21) Diese Regelungen bedeuten ein Mehr an Flexibilität und können - bei geeignetem Vollzug durch untergeordnete Gebietskörperschaften und deren Behörden - allokative Ineffizienzen aufgrund von strikt einzuhaltenden ${ }^{78)}$ Immissionsnormen aufheben. Das zu beobachtende Vollzugsdefizit im Umweltschutz (Mayntz (1978)) läßt jedoch auch bei diesem Vorschlag Skepsis angebracht erscheinen; denn die umweltpolitische Effektivität bundesweit geltender Umweltschutzbestimmungen hängt letztendlich von den wahrgenommenen Ermessensspielräumen der unteren administrativen Einheiten ab, die unmittelbar mit den Anträgen potentieller Emittenten befaßt sind. Von daher ist es angebracht, nach flexibleren Regelungen auf marktwirtschaftlicher Basis zu suchen, die die Rolle von Behörden begrenzen, so daß Vollzugsschwierigkeiten ( $\rightarrow$ Meß- und Kontrollprobleme, Kooperationsdefizite horizontaler und vertikaler Art) weniger oft als Engpaßfaktoren für den Erfolg der Umweltpolitik auftreten.

76) "Die Regelung soll in Belastungsgebieten, in denen bereits die Immissionswerte überschritten sind, den Neubau von Anlagen und deren Anpassung an neue Technologien, Produktionsund Marktverhältnisse erleichtern." (BT-Drucksache 8/2751, S. 8 ).

77) Vgl. z.B. Umwelt (BMI) 69 (1979), S. 14.

78) Bei diesem Punkt befindet sich die Bundesregierung im Gegensatz zur Position der EG-Mehrheit: letztere tritt für generell geltende Immissionsgrenzwerte ein, während die Bundesregierung die Ausnahme von Normüberschreitungen bei gleichzeitig wirksam werdenden Luftreinhalteplänen zulassen möchte. 
(22) Folgende Einzelregelungen seien bisher zusätzlich aufgeführt, um die Nachteile von ins Detail gehenden umweltrechtlichen Bestimmungen im Vergleich zu Konzeptionen herauszustellen, die bei industriellen bzw. gewerblichen Emittenten die Nutzung ihres Innovationspotentials anregen.

(i) In der TA Luft sind z.B. für bestimmte Anlagetypen Höchstwerte für Schwefeldioxidemissionen vorgegeben. Dieser Bestimmung fehlt das dynamische Element, da die Zunahme solcher Emissionsquellen nicht erfaßt wird. Umweltmindestqualitäten in einer Region sind dadurch nicht zwangsläufig auf mittlere bzw. längere sicht gewährleistet. Im Hinblick auf die Umweltrestriktion fehlen selbstregulative Mechanismen innerhalb der industriellen/gewerblichen Emittentengruppe. Wird infolgedessen ein kritisches Immissionsniveau erreicht (oder für die nächste Zukunft erwartet), haben die Behörden gemäß ihrer diskretionären umweltpolitischen Strategie zu intervenieren - z.B. durch Einführung verschärfter Emissionsstandards ${ }^{79)}$ für bestimmte Anlagearten.

(ii) Der "Stand der Technik", von dem die Auflagen ( $\rightarrow$ anlagebezogene Emissionsstandards, (inputbezogene) Produktstandars, Normen für Prouktionsverfahren) abgeleitet werden, beschreibt oft nur Minimalanforderungen. Höhere Anforderungen sind nicht nur technisch realisierbar, sondern können auch von den Vollzugsbehörden verordnet werden, was jedoch selten praktiziert wird (Mayntz (1978)) . - Unabhängig davon fehlt den Emittenten jeder Anreiz, zu einem erhöhten Niveau der Umweltschutztechnologie beizutragen, wenn erst einmal den gesetzten Auflagen genüge geleistet wird. Allenfalls eigene Expansionspläne mögen den einzelnen emittierenden Produzenten veranlassen, nach umweltfreundlicheren Produktionsmethoden zu suchen ( $\rightarrow$ Textziffer 23).

79) So geschehen z.B. in Nordrhein-Westfalen. Vgl. Knoepfel/Weidner $(1980)$, S. 187 . 
(iii) Zusätzliche Kooperationserfordernisse zwischen Behörden, die jeweils für andere Regionen zuständig sind, können aus Ermessensspielräumen erwachsen, über die oberste Landesbehörden im Hinblick auf die Festsetzung von regionalen Immissionskurzzeitwerten bzw. -langzeitwerten verfügen; für bestimmte Gebiete (Belastungsgebiete, Schongebiete) können Ausnahmewerte verfügt werden, die - z.B. mittels größerer Schornsteinhöhen ${ }^{80)}$ - Chancen für den Emissionsexport über die jeweiligen politisch-administrativen Grenzen hinaus eröffnen.

(iv) Die umweltpolitische Effizienz ist ferner durch das Unvermögen der Administration gefährdet, schnell und vorsorgend auf sich abzeichnende Fehlentwicklungen zu reagieren. Zwar ist die Möglichkeit von nachträglichen Änderungsanordnungen für bereits in Betrieb genommene Anlagen gegeben ( $\rightarrow \S 17$ BImSchG), dürfen jedoch nur bei wirtschaftlicher Vertretbarkeit durchgesetzt werden. 81) Die Praktizierbarkeit dieses Konzepts ist davon abhängig, ob es gelingt, den Begriff der wirtschaftlichen Vertretbarkeit operabel auszugestalten. Auf jeden Fall bringt diese Regelung ein beträchtliches Mehr an Verwaltungsaufwand mit sich. Der Zeitaufwand zwischen dem Erkennen der Änderungsnotwendigkeit und dem Vollzug dürfe ebenfalls erheblich sein, was das Risiko von irreversiblen Umweltschäden in sich birgt. - Die Praxis zeigt, daß zwischen Behörden und

80) Die TA Luft enthält Formeln zur Berechnung von Schornsteinmindesthöhen: die Standardformel setzt ideale Diffusionsbedingungen voraus, wobei ein Korrekturfaktor zur Anwendung kommen kann (z.B. bei entsprechender - tatsächlicher oder geplanter - Bebauung); die Mindesthöhe wird auf die Maximalkapazität sowie die relativ umweltschädlichsten Brennstoffinputs und Emissionen bezogen (d.h. daß jeweils die größte aller ermittelten Schornsteinhöhen, die aufgrund von Prognosen für die Einhaltung gebietsbezogener Immissionsgrenzwerte als notwenig erachtet werden, maßgeblich ist.) Neben der damit etablierten Neigung zum Emissionsexport wird an dieser Bestimmung auch kritisiert, daß die maximale Emissionskapazität selbst bei ungünstigen Immissionsverhältnissen (unterhalb der Smogalarm-Schwelle) legal genutzt werden darf.

81) Zu dem Begriff der wirtschaftlichen Vertretbarkeit vgl. Soell $(1980)$. 
Emittenten oft nachträgliche Änderungen kooperativ vereinbart werden. Die Tatsache jedoch, daß es sich dabei zumeist eher um echte Sanierungsfälle als um vorsorgende Strategien handelt (Mayntz et.al.), läßt effektivere Lösungen notwendig erscheinen, worauf im folgenden näher eingegangen werden soll.

(23) Bei der Erstellung von Vorschlägen zur Reduktion von umweltpolitischen Ineffizienzen ist auf Basis der bundesrepublikanischen Verhältnisse davon auszugehen, daß die konturen des bestehenden Umweltrechts bis auf weiteres erhalten bleiben und allenfalls marginale Änderungen $z u$ erwarten sind bzw. möglich erscheinen. Im Zuge einer umfassenden Reform, die "ideale" Emissionszertifikatelösung in der Bundesrepublik etablieren zu wollen, muß - zumindest auf kurze Sicht - als illusorisch bezeichnet werden. Dieses Idealkonzept enthält jedoch einige Komponenten, die auf Grundlage der institutionellen Gegebenheiten in der Bundesrepublik durchaus in die Umweltschutzbestimmungen aufgenommen werden könnten: gemeint sind damit

(i) die zeitliche Begrenzung von Betriebsgenehmigungen für emissionsträchtige Anlagen

und

(ii) die Einführung der Transferierbarkeit dieser Genehmigungen zwischen industriellen/gewerblichen Emittenten. ${ }^{82}$ )

Diese Änderungsvorschläge sind mit Versuchen zu kontrastieren, die festgestellten Vollzugsdefizite über eine Verdichtung des Systems von Rechtsverordnungen und Verwaltungsvorschriften beseitigen $z u$ wollen; denn auflagenorientierte Strategien begünstigen - wie bereits an anderen Stellen dargelegt ${ }^{83)}$ - das Ent-

82) Diese Thesen befinden sich in Ubereinstimmung mit den Empfehlungen von $H$. Bonus (1980).

83) Vgl. z.B. Textziffer 22. 
stehen von überhöhten gesamtwirtschaftlichen Anpassungskosten der Umweltpolitik ( $\rightarrow$ administrative Kosten der Implementierung, Durchführung und Kontrolle; Kosten aufgrund von eingeschränkten Manöver- und Innovationsspielräumen der Produzenten, verursacht durch behördliche Informationsdefizite). Die vorgeschlagenen Regelungen sind ansatzweise bereits im geltenden bzw. geplanten Immissionsschutzrecht enthalten und versprechen von daher Praktikabilität:

Nach $\S 18$ BImSchG kann eine befristet erteilte Genehmigung erlöschen, wenn bestimmte Auflagen zur Emissionsreduktion nicht befolgt werden; auch ein wettbewerbspolitisch unerwünschtes Horten von Genehmigungen soll dadurch ausgeschlossen werden. - Die Möglichkeit, bereits erteilte Genehmigungen nachträglich ändern zu können ( $\rightarrow$ Textziffer 18), kann als eine potentiell zeitlich limitierte Betriebserlaubnis aufgefaßt werden. - Das Zeitmoment ist ferner in $\S 16 \mathrm{BImSChG}$ enthalten; danach besteht für die Betreiber genehmigter Anlagen die Pflicht, sich im Abstand von zwei Jahren zu melden und Bericht über etwaige Abweichungen von den Angaben des Genehmigungsantrages zu erstatten.

Die von der Bundesregierung in ihrem Novellierungsentwurf zum BImSchG vorgeschlagene Sanierungsklausel läßt sich durch geringfügige Modifikationen in eine Regelung zur überbetrieblichen Transferierbarkeit von anlagenbezogenen Emissionsrechten umwandeln. Nach der Sanierungsklausel kann der Betreiber mehrerer Anlagen die Emissionen der alten und geplanten neuen Anlagen einander aufrechnen und unter zeitlichen Auflagen zu die Gesamtemissionen reduzierenden Maßnahmen verpflichtet werden. Indem man dieser Klausel eine überbetriebliche Relevanz zuweist, hat man unter sonst konstanten Bedingungen eine Transferierbarkeit von Emissionsbefugnissen institutionalisiert, die der Dynamik des Wirtschaftsprozesses eher gerecht wird und auch die wirtschaftliche Entwicklung in bestimmten Zonen begünstigen kann.

Beispielsweise können Unternehmen, die sich neu in einer Region mit bereits (fest) realisierten Immissionshöchstwerten ansiedeln 
wollen, einen Teil der Verschmutzungsrechte von ansässigen Produzenten erwerben. Der Zuzug von neuen Industrien mit evtl. umweltfreundlicheren Herstellungsverfahren muß dabei z.B. nicht zwangsläufig an der mangelnden Verhandlungsbereitschaft der Alteingesessenen und/oder an dem sicheren überschreiten der Immissionsgrenzwerte im Fall der Produktionsaufnahme scheitern. In Anlehnung an die vorgesehene Luftreinhalteplanklausel können alte und neue Emittenten einer Region gemeinsam behördlichen Auflagen unterliegen, die auf eine Reduktion der Gesamtemissionen innerhalb eines bestimmten Zeitraumes abzielen. Die betriebs- und anlagenindividuellen Emissionshöchstwerte ergeben sich dann als das Resultat von Verhandlungsprozessen innerhalb der industriellen Emittengruppe. Die Rolle der jeweiligen obersten Landesbehörden kann sich dabei durchaus auf die eines Schiedsgerichtes beschränken. - Von einer solchen Regelung könnten Impulse zur Bildung von regionalen "Umweltkartellen" ausgehen. ${ }^{84)}$ Angesichts der hohen Kosten zur Weiterentwicklung der Umweltschutztechnologie $(\rightarrow$ Textziffer 18) sind Unternehmenskooperationen dieser Art als umweltpolitisch wünschenswert zu beurteilen. ${ }^{85}$ ) Restriktiv wirkende Immissionsorientierungswerte brauchen demnach nicht notwendigerweise auf Dauer gänzlich blockierend auf die Expansion des wirtschaftlichen Aktivitätsniveaus und den strukturellen Wandel in bestimmten Gebieten zu wirken, wenn das Anpassungspotential der emittierenden Produzenten weitgehend erhalten bleibt.

Eine Reform in dem hier vorgeschlagenen Sinn könnte - von evtl. politischen Durchsetzungsschwierigkeiten abgesehen- in der Bundes-

84) Für die USA liegen Beispiele dafür vor, wie Unternehmen untereinander Emissionsrechte handeln ( $\rightarrow$ Ballungsraum L.A.). Vgl. Siebert, H. (1980).

85) Wettbewerbspolitische Einwände mögen darauf hinauslaufen, daß sich die Absprachen zwischen den Unternehmen auf Gebiete ausdehnen können, die nicht unmittelbar den Umweltschutz betreffen. Auf diesen umfangreichen Problemkreis kann hier nicht näher eingegangen werden. Vgl. z.B. Jarée (1978). 
republik auf der Basis des geltenden Rechts ohne nennenswert zusätzlichen Verwaltungsaufwand ${ }^{86)}$ relativ kurzfristig wirksam werden. Die Reglementierungsmöglichkeiten der Umweltbehörden wären im wesentlichen, neben Kontroll- und Sanktionsfunktionen, auf die Vergabe von gebietsspezifischen Immissionsgrenzwerten und die Genehmigungsdauer für den Betrieb der Anlagen beschränkt, würden also nicht mehr auf den Anlagentyp bezogene Emissionswerte umfassen, was merklich reduzierte Informationserfordernisse mit sich bringt. Darüber hinaus sei betont, daß die vorgeschlagenen Regelungen einen Anreiz zur Erhöhung des umweltschutztechnologischen Niveaus ausüben können; denn die Transferierbarkeit von Emissionsrechten begünstigt entscheidend die Vermarktung von technischen Umweltschutzinnovationen - im Gegensatz $z u$ den bestehenden Regelungen, nach denen die Konservierung des herrschenden technischen Niveaus einen Schutz vor schärferen Auflagen darstellt.

86) $\mathrm{Zu}$ erwarten sind dagegen eher niedrigere Kosten.

87) Vgl. dazu auch die Ausführungen im vorstehenden SiebertBeitrag (S. 13-15, $27 \mathrm{f}$ ). 
Literaturverzeichnis :

Adlung, R., Thoroe, C.S. (1980), Neue Wege in der Regionalpolitik, Kieler Diskussionsbeiträge 68 .

BFA/Bundesforschungsanstalt für Landeskunde und Raumordnung (1976), Abwasserabgabe - Instrument der Raumordnung? Informationen zur Raumentwicklung, Heft 8 (1976).

BMBau/Der Bundesminister für Raumordnung, Bauwesen und städteBau (Hrsg.) (1980), Innovationsorientierte Regionalpolitik, Schriftenreihe "Raumordnung", Heft 06.042.

BMBau/Der Bundesminister für Raumordnung, Bauwesen und Städtebau (1973), Raumordnung und Umweltschutz. Entschließung der Ministerkonferenz für Raumordnung von 1972, Bonn.

BMI/Bundesministerium des Innern. (Hrsg.), Umwelt. Informationen des BMI zur Umweltplanung und zum Umweltschutz.

BMI/Bundesministerium des Innern (1980), Was Sie schon immer über Umweltschutz wissen sollten, Stuttgart, Berlin, Köln, Mainz.

Bonus, H. (1980), Ein ökologischer Rahmen für die Soziale Marktwirtschaft, Wirtschaftsdienst III, 141-146.

Bullinger, M. (1974), Umweltrechtliches Verursacherprinzip und Raumordnung, in: Festschrift für Werner Weber, Berlin.

Council on Environmental Quality (1975), Environmental Quality, the sixth annual report, Washington D.C..

Deutscher Bundestag (1971), Gesetz über die Gemeinschaftsaufgabe "Verbesserung der regionalen Wirtschaftsstruktur" vom 6. Oktober 1969, zuletzt geändert durch das Gesetz zur Änderung der Gesetze über die Gemeinschaftsaufgabe vom 23.12.1971, Bonn.

Deutscher Bundestag (1978), Bundestags-Drucksache 8/2006: 1. Immissionsschutzbericht der Bundesregierung, Bonn.

Deutscher Bundestag (1978), Bundestagsdrucksache 8/2014: Siebenter Rahmenplan der Gemeinschaftsaufgabe "Verbesserung der regionalen Wirtschaftsstruktur", Bonn.

Deutscher Bundestag (1979), Bundestagsdrucksache 8/2751: Entwurf eines Zweiten Gesetzes zur Änderung des Bundes-Immissionsschutzgesetzes, Bonn.

Fishelson, G. (1979), Political and Jurisdictional Systems for Regional Environmental Policy (Discussion), in: Siebert/Walter/Zimmermann (eds.).

Freistaat Bayern (1978), Umweltpolitik in Bayern.

Fürst, D., Klemmer, P., Zimmermann, K. (1976), Regionale Wirtschaftspolitik, Tübingen, Düsseldorf.

IfU/Institut für Umweltschutz (Hrsg.) (1978), Möglichkeiten und Grenzen kommunalen Umweltschutzes, Berlin.

Jarée, J. (1978), ökonomische Interdependenzen zwischen Umweltschutz und Wettbewerb, in: Zeitschrift für Umweltpolitik, 1. Jg., $71-94$.

Kabelitz, K.R. (1980), Umweltpolitik als Beschäftigungspolitik? In: Wirtschaftsdienst $I, 36-43$.

Knoepfel, P., Weidner, H. (1980), Handbuch der $\mathrm{SO}_{2}$-Luftreinhaltepolitik. Teil II: Länderberichte (Teil I: Vergleichende Analyse), Berlin.

Kuhl, G. (1977), Umweltschutz im materiellen Raumordnungsrecht, Münster. 
Land Hessen, Landesentwicklungsplan "Hessen'80" .

Maier-Rigaud, G. (1980), Umweltpolitik in der Marktwirtschaft, Wirtschaftsdienst VII, 341-345.

Mayntz, R. et.al. (1978), Vollzugsprobleme der Umweltpolitik

(hrsg. vom Rat von Sachverständigen für Umweltfragen), Stuttgart.

Meißner, W. (1980), Umweltpolitik und Beschäftigung. Bemerkungen zum Aufsatz von Kabelitz. In: Wirtschaftsdienst I, 44-45.

Meißner, W. (1979), Umweltpolitik und Beschäftigung, in: Wirtschaftsdienst VII, 330-335.

Meißner, W., Hödl, E. (1976), Positive ökonomische Aspekte des Umweltschutzes. Gutachten für den Bundesminister des Innern.

Meyer-Dohm, P., Tokarski, W. (1980), Regionale Innovations- und Transferpolitik. Möglichkeiten einer innovationsorientierten Regionalpolitik für das Ruhrgebiet, in: Wirtschaftsdienst XI, 558-565.

OECD-Observer $\mathrm{Nr} .97 / 1979$.

Rheinland-Pfalz/Ministerium für Soziales, Gesundheit und Umwelt(MSGU) (1980), Luftreinhalteplan 1979-1984 Ludwigshafen-Frankenthal. Mainz.

Rheinland-Pfalz/Ministerium für Soziales, Gesundheit und Sport (1978), Luftreinhaltung in Rheinland-Pfalz, Mainz.

Siebert, H. (1979), The Regional Dimensions of Environmental Policy, in: Siebert/Walter/Zimmermann, 1-12.

Siebert, H. (1978), Ökonomische Theorie der Umwelt, Tübingen.

Siebert, H. (1978a), Voerde und eine neue Umweltpolitik, Wirtschaftsdienst, 85. Jg., 36-40.

Siebert, H., Walter, I., Zimmermann, K. (eds.) (1979), Regional Environmental Policy, New York, London.

Siebert, H. (unter Mitarbeit von W. Vogt) (1976), Analyse der Instrumente der Umweltpolitik, Göttingen.

Soell, H. (1980), Der Grundsatz der wirtschaftlichen Vertretbarkeit im Bundesimmissionsschutzgesetz, Tübingen.

SPD-Thesen zur Umweltpolitik, in: Umwelt 1975, Heft 2 .

Sprenger, R.U., Britschkat, G. (1980), Umweltschutz und sektoraler Strukturwandel, München.

Sprenger, R.U., Britschkat, G. (1979), Beschäftigungseffekte der Umweltpolitik, Berlin-München.

SRU/Der Rat von Sachverständigen für Umweltfragen (1978), Umweltgutachten 1978 .

Stamer, P. (1976), Niveau-u.strukturorient.Umw.politik, Göttingen UBA/Umwel tbundesamt (1977), Materialien z.Imm.schutzbericht 1977 . Ullmann, A.A., Zimmermann, K. (1980), Strukturelle Aspekte des Umweltschutzes: Die Umweltschutzindustrie, in: Wirtschaftsdienst $\mathrm{X}$, 508-515.

Umlauf, J. (1972), Zum Verhältnis von Umweltschutz und Raumordnung, in: Raumforschung und Raumordnung, 195-199.

Walter, I. (1973), Environmental Control and the Pattern of International Trade and Investment: An Emerging Policy Issue. In: Baldwin, R.E., Richardson, J.D. (eds.), Selected Topics in International Trade and Finance, Boston.

WZB/Wissenschaftszentrum Berlin (1980), WZB-Mitteilungen Nr. 12 . 
II. Bestimmung der anzustrebenden Umweltqualität

Horst Siebert - 978-3-631-75607-2

Downloaded from PubFactory at 01/11/2019 03:09:22AM

via free access 
Horst Siebert - 978-3-631-75607-2

Downloaded from PubFactory at 01/11/2019 03:09:22AM

via free access 
The value of environmental quality

\author{
by \\ Pierre Dehez \\ University of Mannheim \\ and \\ CORE, University of Louvain
}

\title{
1. Introduction
}

The present paper is concerned with the following question: How much should society spend to improve the quality of the environment? Clearly, the environment is a public good ${ }^{1}$ and therefore, the natural framework in which to answer the question raised above is the "Pure Theory of Public Expenditures" as developed by Samuelson (1954). This theory describes the conditions for an optimal - or efficient - provision of public goods and, when applied to environmental quality, it implicitely defines the value that society places upon the environment.

According to this theory, efficiency requires that a public good should be produced up to the point where the cost of producing an extra unit, measured in terms of some numéraire commodity, coincides with the total amount that the agents of the

1) A (pure) public good is a commodity such that the consumption by any agent of a given unit of it does not preclude the simultaneous consumption of the same unit by other agents. Formally, a public good is therefore defined by $x^{i} \leq y$ for all $i$, where $x^{i}$ denotes the consumption of agent $i$ and $y$ is the available quantity. It is opposed to a private good which is defined by $\Sigma x^{i} \leq y$. 
economy, taken together, would just be willing to sacrifice for an extra unit of the public good. In other words, the marginal cost of producing the public good should be equal to the sum over all the agents of the marginal rates of substitution between the public good and the numéraire, i.e. the marginal willingnesses to pay for the public good. This is the basic criterion for guiding an efficient production of public goods. ${ }^{2}$ The quality of the environment has all the properties of a public good and therefore, as far as efficiency is concerned, the management of the environment should be based on this criterion.

In order to illustrate how this theory can be used to determine the optimal quality of the environment we assume that the level of quality can be measured by some scalar q. This assumption is made to simplify the analysis. Indeed, we could have taken $q$ to be a vector. What is important, is that $q$ must be directly relevant for the agents concerned. ${ }^{3}$ Let $\mathrm{N}$ denote the set of agents, each agent $i \in N$ being characterized by his marginal willingness to pay $\varphi^{i}(q)$ as a function of environmental quality. Let us define by $c(q)$ the marginal cost of main-

2) On the other hand, a private good should be produced up to the point where the cost of producing an extra unit coincide with the amount that each agent would just be willing to sacrifice for an extra unit of the private good. Hence, if $\varphi^{i}$ denotes the marginal willingness to pay and $c$ the marginal cost, we have $\varphi^{i}=c \forall i$ for a private good and $\Sigma \varphi^{i}=c$ for a public good.

3) Hence, we do not explicitely discuss the problem of the optimal level of pollution which is related to $q$ via some damage function, a concept defined in Mäler (1975) and in Mäler and Wyzga (1976). 
taining environmental quality at a level $\mathrm{q}$. The theory tells us that an optimal level of environmental quality must satisfy the following equation

$$
\sum_{i \in N} \varphi^{i}(q)=c(q)
$$

where the left-hand side can be interpreted as the value - or the price - that society places upon the environment.

The aim of the present paper is precisely to investigate the problem of estimating this value. This will be done in Section 3 where alternative methods will be discussed. Section 2 is devoted to the review of the model of consumer behaviour within which the concept of marginal willingness to pay is defined.

2. The marginal willingness to pay: a formalization

2. 1 The model

Let us consider a particular agent. There are $\ell$ (private) goods and he has preferences defined over vectors $(x, q) \in R_{+}^{\ell+1}$ These preferences are assumed to be represented by a utility function $u$ defined over $R_{+}^{\ell+1}$, strictly increasing in its $\ell+1$ arguments, strictly quasi-concave and twice continuously differentiable. Hence, we have $\partial u / \partial x_{h}>0$ for all $h=1, \ldots, l$ and $\partial u / \partial q>0$. The price vector is denoted by $p$ and we take the first commodity as numéraire. Hence, $p_{1}=1$ and we assume that $p_{h}>0, h=2, \ldots, l$. 
For a given level of environmental quality $q$, the agent chooses the vector $x$ which maximizes his utility $u(x, q)$ subject to the budget constraint $\sum p_{h} x_{h} \leq w$, where $w$ denotes $h$ is wealth. Assuming an interior solution, the equations

$$
\frac{\partial u}{\partial x_{h}}=p_{h} \frac{\partial u}{\partial x_{1}} \quad h=2, \ldots l l
$$

$$
\sum_{h=1}^{\ell} p_{h} x_{h}=w
$$

define the (usual) demand functions 4

$$
x_{h}=f_{h}(w, q) \quad h=1, \ldots, l
$$

The corresponding level of utility is given by

$$
v(w, q)=u(f(w, q), q)
$$

This defines the indirect function which represents the preferences of the agent between wealth and environmental quality. Given the assumptions made on the (direct) utility function, the demand functions are continuously differentiable and the indirect utility function is twice continuously differentiable. Furthermore, $v$ is a strictly increasing function in both its arguments. Indeed, using the equations (2), we have successively

$$
\frac{\partial v}{\partial w}=\sum_{h=1}^{\ell} \frac{\partial u}{\partial x_{h}} \cdot \frac{\partial f_{h}}{\partial w}=\frac{\partial u}{\partial x_{1}} \cdot \sum_{h=1}^{\ell} p_{h} \frac{\partial f_{h}}{\partial w}
$$

and

$$
\frac{\partial v}{\partial q}=\sum_{h=1}^{\ell} \frac{\partial u}{\partial x_{h}} \cdot \frac{\partial f_{h}}{\partial q}+\frac{\partial u}{\partial q}=\frac{\partial u}{\partial x_{1}} \sum_{h=1}^{\ell} p_{h} \frac{\partial f_{h}}{\partial q}+\frac{\partial u}{\partial q}
$$

But, from equation (3), we also have

4) The price system being fixed, we omit it from the arguments of the functions. 
(4)

$$
\sum_{h=1}^{\ell} p_{h} \frac{\partial f_{h}}{\partial w}=1
$$

and

$$
\sum_{h=1}^{\ell} p_{h} \frac{\partial f_{h}}{\partial q}=0
$$

Hence the derivatives of $\mathrm{v}$ with respect to $\mathrm{w}$ and to $\mathrm{q}$ are given by

$$
\begin{aligned}
& \frac{\partial v}{\partial w}=\frac{\partial u}{\partial x_{1}} \\
& \frac{\partial v}{\partial q}=\frac{\partial u}{\partial q}
\end{aligned}
$$

From the equation (5) we see that, following a change in environmental quality, a shift in the demand for one good has to be compensated by a shift in the demand for other goods going in the opposite direction. Furthermore, the effect of a change in environmental quality is related to the substitution effect of price changes. Indeed, as it is shown in Barten (1977), we have

$$
\frac{\partial f_{h}}{\partial q}=\frac{-1}{\lambda} \sum_{j=1}^{\ell} k_{h j} \frac{\partial^{2} u}{\partial x_{j} \partial q}
$$

where $\lambda$ is the marginal utility of money defined in equation (6) and $k_{h j}$ is the substitution effect between the goods $h$ and j.

The dual problem consists in minimizing the expenditures $\Sigma p_{h} x_{h}$ subject to the utility constraint $u(x, q) \geq \bar{u}$. The equation (2) together with the equation

$$
u(x, q)=\bar{u}
$$


define the compensated demand functions

$$
x_{h}=\tilde{f}_{h}(\bar{u}, q) \quad h=1, \ldots, l
$$

The minimum wealth necessary to achieve a level $\bar{u}$ of utility is therefore given by

$$
m(\bar{u}, q)=\sum_{h=1}^{\ell} p_{h} \tilde{f}_{h}(\bar{u}, q)
$$

which defines the expenditure function. Alternatively, this function can be obtained as the solution of the equation $v(w, q)=\bar{u}$ in terms of $w, i$.e. we have $v(m(\bar{u}, q), q)=\bar{u}$. Given the assumptions made on $u$, the functions $\tilde{f}_{h}$ and $m$ are continuously differentiable in $\bar{u}$ and $q$.

2.2 The benefit from an improvement in environmental quality

Let us consider an improvement in the quality of the environment from $q_{0}$ to $q_{1}$. There are two different measures of the benefit for an agent from such an improvement, the compensating variation and the equivalent variation. ${ }^{5}$ The compensating variation (CV) is the amount that the agent is willing to forfeit while staying on the same indifference surface. The equivalent variation (EV) is the amount by which the agent's wealth must be adjusted so as to make him as badly off without the change as he would be with it.

Formally, let $\bar{u}_{0}=v\left(w, q_{0}\right)$ and $\bar{u}_{1}=v\left(w, q_{1}\right)$ denote the levels of utility before and after the change in environmental

5) See for example Mishan (1971). 
quality. Then, we have

$$
C V=m\left(\bar{u}_{0}, q_{0}\right)-m\left(\bar{u}_{0}, q_{1}\right)=w-m\left(\bar{u}_{0}, q_{1}\right)
$$

and

$$
E V=m\left(\bar{u}_{1}, q_{0}\right)-m\left(\bar{u}_{0}, q_{0}\right)=m\left(\bar{u}_{1}, q_{0}\right)-w
$$

Hence, in the $(w, q)$ plane we have

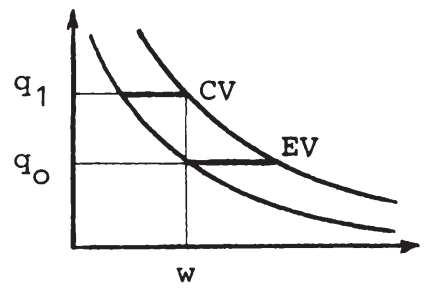

These measures are different approximations of the gain in utility from an improvement in environmental quality. They are used in cost-benefit analysis, and the problem to decide which measure to use is discussed by Mäler (1971) and by Mäler and Wyzga (1976). These measures coincide for infinitesimal change in $q$ and they define the marginal willingness to pay which can be interpreted as the value that the agent places upon the environmental quality. It is given by

$$
\varphi(w, q)=-\frac{\partial m}{\partial q}
$$

and corresponds to the slope of the indifference curve $\mathrm{v}(\mathrm{w}, \mathrm{q})=\overline{\mathrm{u}}$.

This quality can be characterized alternatively in terms of the (direct) utility function or in terms of the indirect utility function. Indeed, since $v(m(\bar{u}, q), q)=\bar{u}$, we have

$$
\frac{\partial v}{\partial w} \cdot \frac{\partial m}{\partial q}+\frac{\partial v}{\partial q}=0
$$


and therefore, the marginal willingness to pay defined in (9) can be written as

$$
\varphi(w, q)=\frac{\partial v / \partial q}{\partial v / \partial w}
$$

i.e. as the marginal rate of substitution between wealth and environmental quality. Using (6) and (7), it can also be written as

$$
\varphi(w, q)=\frac{\partial u / \partial q}{\partial u / \partial x_{1}}
$$

i.e. as the marginal rate of substitution between the numeraire and environmental quality. By the assumption of strict quasiconcavity of $u, \varphi$ is a strictly decreasing function of $q$. Hence, using the definition of $\varphi$ given in (10), we can conclude that the indirect utility function $v$ is also strictly quasi-concave and that $\varphi$ is strictly increasing in $w$. Therefore, the value an agent places upon the environment is an increasing function of his wealth and a decreasing function of the quality of the environment.

We now turn to the problem of estimating this value.

3. The marginal willingness to pay: its empirical measure

\subsection{Position of the problem}

We are interested in estimating the value that society places upon the quality of the environment as defined by the sum of the marginal willingnesses to pay $\Sigma \varphi^{i} \cdot\left(w^{i}, q\right)$. Two types of approach are available. On the one hand, a direct approach 
which consists in asking directly to the agents concerned (or to a sample of them) how much they are willing to pay for a small improvement in the environmental quality. On the other hand, an indirect approach which consists to exploit the relations existing between the quality of the environment and the consumption of some private goods.

The direct approach comes up against the well known "freerider" problem. This is the problem of the incentives for a correct revelation of preferences. Indeed, either the revealed information is used to assess the contribution of each agent in the financing of the improvement in environmental quality, in which case there is an incentive for under-reporting the willingness to pay; or the revealed information is not directly related to a financial contribution, in which case there is an incentive for over-reporting the willingness to pay. We will not deal here with the direct approach. Let us just mention the work of Kurz (1974). Under the assumption that "In the absence of any reward or loss due to the revelation of true preferenes, individuals have the intrinsic desire to tell the truth ..." (p. 333), he proposes different schemes allowing to a control of the incentives for incorrect revelation of preferences. Either the agents are placed in a setting which lead them to reveal the truth, or they are asked to provide further information allowing to an estimation of the bias they have introduced in their answers. Although the assumption of "goodwill" on which this analysis is based is rather strong, the experimental re- 
sults obtained by Bohm (1972) seem to suggest that it is not such an unreasonable assumption.

The indirect approach relies on the assumption that there exists a subset of private goods the consumption of which is related to the environment. More specifically, it is assumed that the preferences of an agent can be defined over some private goods and a "final" consumption good, called "recreation", which is "produced" by combining the other private goods with the quality of the environment. It is an application of the concept of "technology of consumption" introduced by Lancaster (1966) and Muth (1966) and extended by Sandmo (1973) to cover the case where there are public goods. It is this extension which is followed here.

3.2 The indirect approach

Let us assume that there exists a commodity, called "recreation", which is "produced" by combining the quality of the environment with some private goods. ${ }^{6}$ Let $H \subset\{1, \ldots, l\}$ denote this subset of private goods. We assume furthermore that the preferences of the agent can be represented by the following weakly separable utility function

$$
u(x, q)=\tilde{u}\left((x)_{1}, \psi\left((x)_{2}, q\right)\right)
$$

6) A typical final good is the quantity of recreation consumed by the agents. For example, Nemerow (1970) uses the "recreationday" as a unit of measure. The use of a scalar is a simplifying assumption but we could as well have used a vector with recreation, health, and so on ... as components. 
where $\mathrm{x}=\left((\mathrm{x})_{1},(\mathrm{x})_{2}\right)$ and $(\mathrm{x})_{2}$ is a vector with $|\mathrm{H}|$ components. Hence, the preferences of the agents are defined over the private goods $h \in\{1 \ldots l\} / H$ and a final good. The functions $\tilde{u}$ and $\psi$ are assumed to be twice continuously differentiable, strictly increasing and strictly quasi-concave. Here the function $\psi$ can be interpreted as a production function in the sense of the neoclassical theory of the firm, i.e. $y=\psi\left((x)_{2}, q\right)$ where $\left((x)_{2}, q\right)$ is the vector of inputs and $y$ is the output.

With this formulation, the marginal utility of environmental quality can be written as

$$
\frac{\partial u}{\partial q}=\frac{\partial \tilde{u}}{\partial y} \cdot \frac{\partial \psi}{\partial q}
$$

while the marginal utility of a good $h \in H$ is given by

$$
\frac{\partial u}{\partial x_{h}}=\frac{\partial \tilde{u}}{\partial y} \cdot \frac{\partial \psi}{\partial x_{h}}
$$

Using equation (2) and the definition of the marginal willingness to pay given in (11), we get

$$
\varphi(w, q)=p_{h} \frac{\partial \psi / \partial q}{\partial \psi / \partial x_{h}}
$$

for some $h \in H$. Hence, $\varphi$ appears as the value of an "objective" marginal rate of substitution and, in order to estimate it, it is then necessary to estimate the technology of recreation as described by the production function $\psi$.

We will now give a few examples to illustrate how this concept can be used to obtain an estimate of the marginal willingness to pay for an improvement in environmental quality. 
Example 1 Let us assume that the technology of recreation is described by the following Cobb-Douglas production function

$$
y=q^{\alpha} \prod_{h \in H} x_{h}^{\beta h}
$$

with $0<\alpha<1,0<\beta_{h}<1$ for all $h \in H$ and $\alpha+\sum \beta_{h} \leq 1$. We have successively

$$
\frac{\partial \psi}{\partial q}=\alpha \frac{y}{q}
$$

and

$$
\frac{\partial \psi}{\partial x_{h}}=\beta_{h} \frac{y}{x_{h}}
$$

Furthermore, for $h \in H$, the equation (2) can be written as

$$
\frac{\partial \tilde{u}}{\partial y} \cdot \frac{\partial \psi}{\partial x_{h}}=p_{h} \frac{\partial u}{\partial x_{1}}
$$

or, alternatively,

$$
\beta_{h} y \frac{\partial \tilde{u}}{\partial y}=\frac{\partial u}{\partial x_{1}} p_{h} x_{h}
$$

for $h \in H$. Summing over all $h \in H$, we obtain the following expression

$$
y \frac{\partial \tilde{u}}{\partial y} \sum_{h \in H} \beta_{h}=\frac{\partial u}{\partial x_{1}} \bar{w}
$$

where

$$
\bar{w}=\sum_{h \in H} p_{h} x_{h}
$$

defines the total expenditures related to the environment and its quality. From the definition of the marginal willingness to pay given in (11), we have

$$
\varphi(w, q)=\frac{\partial \tilde{u}}{\partial y} \cdot \frac{\partial \psi / \partial q}{\partial u / \partial x_{1}}
$$


Therefore, using the equation (14), we finally get

$$
\varphi(w, q)=\gamma \frac{\bar{w}}{q}
$$

where

$$
\gamma=\frac{\alpha}{h \in H}
$$

is a coefficient which measures the relative weight of the environment with respect to the private goods in the production of recreation. Given that $\varphi$ can be interpreted as a price, we have the following equation

$$
\varphi(w, q) \cdot q=\gamma \bar{w}
$$

which says that the monetary value of the environment is proportional to the expenditures on recreation.

In the particular case where $\gamma=1$, the equation (15) gives a theoretical justification to the measurement methods which consider the expenditures of the agents on recreation as a good approximation of the monetary value of the environment. 7

Example 2 Let us assume that the technology of recreation is described by the following production function

$$
y=\prod_{h \in H}\left(x_{h}-\bar{x}_{h}\right)^{\beta} h \quad\left(x_{h} \geq \bar{x}_{h}\right)
$$

where $0<\beta_{h}<1$ and $\sum_{h \in H} \beta_{h} \leq 1$. Here, $\bar{x}_{h} \geq 0$ denotes the minimum quantity of private good $h$ which is necessary to produce the final good. We assume that these quantities are linear

7) Such a measure is used by Nemerow (1970) to estimate tine monetary value of the Lake Onondaga (USA). 
functions of the environmental quality, i.e.

$$
\bar{x}_{h}=\max \left(0, \alpha_{0}-\alpha_{h} q\right)
$$

For simplicity, let us assume that $\alpha_{0}-\alpha_{h} q \geq 0$ for all $h \in H$. It is easily verified that the following relation holds ${ }^{8}$

$$
\frac{\partial \psi}{\partial q}=\sum_{h \in H} \alpha_{h} \frac{\partial \psi}{\partial x_{h}}
$$

On the other hand, multiplying both sides of (13) by $\alpha_{h}$ and summing over all $\mathrm{h} \in \mathrm{H}$, we get

$$
\frac{\partial \tilde{u}}{\partial y} \sum_{h \in H} \alpha_{h} \frac{\partial \psi}{\partial x_{h}}=\frac{\partial u}{\partial x_{1}} \cdot \sum_{h \in H} \alpha_{h} p_{h}
$$

Combining the last two relations, we obtain the following expression

$$
\frac{\partial \tilde{u}}{\partial y} \cdot \frac{\partial \psi}{\partial q}=\frac{\partial u}{\partial x_{1}} \sum_{h \in H} \alpha_{h} p_{h}
$$

whose the left hand side is precisely equal to $\partial \mathrm{u} / \partial \mathrm{q}$. Hence, using the definition of $\varphi$ given in (11), we finally get

$$
\varphi(w, q)=\sum_{h \in H} \alpha_{h} p_{h}
$$

i.e. the marginal willingness to pay is a linear combination of the prices of the private goods and is independent of the level of environmental quality and of wealth (see footnote 8 ).

Using the equilibrium conditions (2) and (3), it is easily verified that the demand functions are given by

$$
\mathrm{x}_{\mathrm{h}}=\overline{\mathrm{x}}_{\mathrm{h}}+\frac{\gamma_{\mathrm{h}}}{\mathrm{p}_{\mathrm{h}}}\left(\overline{\mathrm{w}}-\sum_{\mathrm{k} \in \mathrm{H}} \mathrm{p}_{\mathrm{k}} \overline{\mathrm{x}}_{\mathrm{k}}\right)
$$

8) Hence, the function $\psi$ defined here is only strictly quasi-concave in $x_{2}$ for given $q$ and not jointly in $\left(x_{2}, q\right)$. This explains why we obtain a constant marginal willingness to pay in which case the indifference curves in the $(w, q)$ plane are straight lines. 
where

$$
\gamma_{h}=\frac{\beta_{h}}{\sum_{k \in H} \beta_{k}}
$$

The demand functions can also be written in terms of the expenditures, i.e.

$$
p_{h} x_{h}=\eta_{o h}+\eta_{1 h} q+\gamma_{h} \bar{w} \quad(h \in H)
$$

where

$$
n_{\mathrm{oh}}=\alpha_{\mathrm{o}}\left(\mathrm{p}_{\mathrm{h}}-\gamma_{\mathrm{h}} \sum_{\mathrm{k} H} \mathrm{p}_{\mathrm{k}}\right)
$$

and

$$
\eta_{1 h}=\gamma_{h} \sum_{k \in H} \alpha_{k} p_{k}-\alpha_{h} p_{h}
$$

Let us assume that we have sufficient data to estimate the system of equations (16) and, let $\hat{n}_{\mathrm{oh}}, \hat{n}_{1 \mathrm{~h}}$ and $\hat{\gamma}_{\mathrm{h}}$ denote the estimators so obtained. It is immediately seen that the following system of equations

$$
\hat{n}_{1 h}=\hat{p}_{h} \varphi-\alpha_{h} p_{h}
$$

has no solution in $\varphi$ whenever we impose the adding-up condition

$$
\sum_{h \in H} \hat{\gamma}_{h}=1
$$

It is nevertheless possible to get an estimate of $\varphi$ if there exists one private good $k \in H$ such that $\alpha_{k}=0$, i.e. such that the minimum quantity $\bar{x}_{k}$ necessary to produce the final good does not depend on the environmental quality. ${ }^{9}$ Indeed, in that

9) The equipment used for fishing is an example of a commodity whose minimum quantity is independent of the water quality. But, as quality decreases, more equipment is needed. This has been shown by Stevens (1966) in a study where the quality of the water is measured by the "angling success per unit of effort." 
case, it is only necessary to estimate the demand function for that commodity and an estimate of the marginal willingness to pay is given by $\hat{\varphi}=\hat{n}_{1 \mathrm{k}} / \hat{\gamma}_{\mathrm{k}}$.

Before going to the third example, notice that the weak separability of the utility function allows us to consider the following dual problems

$$
\text { Max } y \quad \text { subject to } \sum_{h \in H} p_{h} x_{h} \leq \vec{w}
$$

and

$$
\text { Min } \sum_{h \in H} p_{h} x_{h} \quad \text { subject to } \quad y \geq \bar{y}
$$

for given $\bar{w}$ and $\bar{y}$. Let us denote by $g_{h}(\bar{w}, \bar{p}, q)$ and $\tilde{g}_{h}(\bar{y}, \bar{p}, q)$ the respective solutions of these problems, where $\bar{p}=\left(p_{h} \mid h \in H\right)$. Using the same arguments as in Section 2, the marginal willingness to pay is given by $-\partial \bar{m} / \partial q$ where

$$
\bar{m}=\sum_{h \in H} p_{h} \tilde{g}_{h}(\bar{y}, \bar{p}, q)
$$

is the minimum wealth needed to produce a quantity $\bar{y}$ of final good.

Example 3

Let us assume that there exists a private good $k \in H$ such that the agent is indifferent about environ'ntal quality whenever he does not consume of that good, i.e. hat

$$
\frac{\partial \psi}{\partial q} \equiv 0 \quad \text { whenever } \quad x_{k}=0
$$

Then, if one has an estimated demand function $\hat{g}_{k}(\bar{w}, \bar{p}, q)$ for that good, such that $\partial \hat{g}_{\mathrm{k}} / \partial \mathrm{q} \neq 0$, Mäler shows that (17) is a 
sufficient condition to derive an estimate of the expenditure function $\bar{m}$ and thus, of the marginal willingness to pay.

The condition (17) is rather strong and one can imagine just a few cases where it would be fulfilled. The case of fishing equipment is an example as far as water quality is concerned. The same remark applies to the condition used in the Example 2. The (second) best approach seems to be given by Example 1 where the sum over all the agents of their expenditures on the final good can be used to estimate the monetary value of environmental quality, i.e. $q \sum p^{i}=\Sigma \bar{w}^{i}$. Hence, one has to observe by how much the expenditures on recreation change when the quality of the environment changes ${ }^{10}$ and an estimate of $\sum \varphi^{i}$ is then given by $\Delta \bar{w} / \Delta q$, with $\bar{w}=\Sigma \bar{w}^{i}$.

10) One can also compare two situations differing only in the quality of the environment. This is the way Nemerow (1970) proceeds to estimate the value of Lake Onondaga. 
References:

Barten, A.P. (1977), The Systems of Consumer Demand Functions Approach: A Review, Econometrica, 45, 23-51.

Bohm, P. (1972), Estimating Demand for Public Goods: An Experiment, European Economic Review, 3, 111-130.

Kurz, M. (1974), Experimental Approach to the Determination of the Demand for Public Goods, Journal of Public Economics, 3, $329-348$.

Lancaster, K.J. (1966), A New Approach to Consumer Theory, Journal of Political Economy, 74, 132-157.

Mäler, K.G. (1971), A Method of Estimating Social Benefits from Water Pollution Control, Swedish Journal of Economics, 73, $106-118$.

Mäler, K.G. (1975), Damages Functions and their Estimation: a Theoretical Survey, in Environmental Damage Costs, OCDE, Paris.

Mäler, K.G. and Wyzga, R. (1976), Economic Measurement of Environmental Damage, OCDE, Paris.

Mishan, E.J. (1971), Cost-Benefit Analysis, Allen and Unwin, London.

Muth, R.F. (1966), Household Production and Consumer Demand Functions, Econometrica, 34, 699-708.

Nemerow, N.E. (1970), Benefits from Water Quality Enhencement, Environmental Protection Agency, Washington.

Samuelson, P.A. (1954), The Pure Theory of Public Expenditures, Review of Economics and Statistics, 36, 387-389.

Sandmo, A. (1973), Public Goods and the Technology of Consumption, Review of Economic Studies, 40, 517-528.

Stevens, J.B. (1966), Recreation Benefits from water Pollution Control, Water Resources Research, 2, 167-182. 
Die Allokation lokaler öffentlicher Güter

bei Konsumentenmobilität.

- Einige Anmerkungen zum Tiebout-Theorem -

Ferdi Dudenhöffer *

Helga Gebauer

1. Einleitung

Vor mehr als zwei Jahrzehnten hat C.M. Tiebout (1956) die Behauptung aufgestellt, er habe einen institutionellen Rahmen gefunden, in dem lokale öffentliche Güter optimal angeboten werden. Dieses sogenannte "Tiebout-Theorem" wird seither viel diskutiert und in seiner Allgemeingültigkeit bestritten. In der vorliegenden Arbeit wird diese Diskussion aufgegriffen und anhand von Modellen Kritik an Tiebouts Hypothese geübt. Im folgenden wird noch einmal das ursprüngliche Tiebout-Modell kurz dargestellt. Im 2. Teil beschäftigen wir uns dann mit dem problem der Charakterisierung lokaler öffentlicher Güter und im 3. Teil wird die Effizienz des Tiebout'schen "voting with one's feet"Konzepts bei alternativen Informationsausstattungen der Konsumenten untersucht. Ein Vergleich der Finanzierung von lokalen öffentlichen Gütern durch Kopfsteuer mit der Einkommensteuerfinanzierung zeigt Allokationsvorteile der Kopfsteuer.

* Für kritische Hinweise und Anregungen danken wir Prof. Dr. Horst Siebert 
1. 1 Das Tiebout-Mode11

Tiebout greift in seinem Artikel "A Pure Theory of Local Expenditures" (Tiebout 1956)) eine Aussage von Musgrave (1939, 1955) und Samuelson (1954, 1955) auf, die behaupten, daß keine "Marktlösung" für Ausgaben für öffentliche Güter existiere. Tiebout bestreitet dies für lokale öffentliche Güter.

Bei seinen tberlegungen geht Tiebout (1956) von einer Modellökonomie mit privaten und lokalen öffentlichen Gütern aus. Die verschiedenen Gemeinden der ökonomie offerieren den einzelnen Konsumenten Bündel an öffentlichen Gütern, die nur dann konsumiert werden können, wenn der Konsument in der entsprechenden Gemeinde wohnt. Werden die Angebote an öffentlichen Gütern in jeder Gemeinde ausschließlich aus Steuern der Bewohner dieser Gemeinde. finanziert, steht, in Analogie zu privaten Gütermärkten, der "Tiebout'sche" Konsument verschiedenen Anbietern (Gemeinden) 10kaler öffentlicher Güter gegenüber. Ein rational handelnder Konsument wählt daher aus der "Angebotspalette" öffentlicher Güterund steuerkombinationen über eine "voting with one's feet"-Entscheidung diejenige Gemeinde, die seine präferenzen maximiert. Ein Gleichgewichtszustand ist dann erreicht, wenn kein konsument mehr von einer Gemeinde in eine andere wandert. Dieser Gleichgewichtszustand ist Pareto-optimal, da jeder Konsument in die Gemeinde wandert, deren Güter- und Steuerpaket seine Präferenzen maximiert und er damit seine tatsächliche Zahlungsbereitschaft für die lokalen öffentlichen Güter enthüllt. Diesem Ansatz liegen folgende, explizit formulierte Annahmen zugrunde:

(I) Vollkommene Mobilität der Konsumenten.

(II) Vollkommene Information der Konsumenten über die Einnahme und Ausgabe-Strukturen der Gemeinden.

(III) Große Anzahl von Gemeinden.

(IV) Alle Individuen leben von Dividendeneinkommen.

(V) Keine externen Effekte der öffentlichen Leistungen zwischen Gemeinden.

(VI) Für jede struktur der öffentlichen Leistungen und für jede Präferenz-Struktur exisitert eine optimale Gemeindengröße. 
(VII) Jede Gemeinde strebt ihre optimale Gemeindegröße an. Damit hat Tiebout für ökonomien mit lokalen öffentlichern Gütern ein dezentrales, nicht-diktatorielles Allokationsverfahren beschrieben, das paretooptimale Allokationen erzeugt.

1.2 Einige ausgewählte Kritikpunkte ${ }^{1)}$

Die Allgemeinheit der Annahmen des Tiebout-Konzepts bestimmt seinen Anwendungsbereich', deshalb soll hier eine kurze prämissenkritik geübt werden.

Die zentralen Annahmen des Tiebout-Mechanismusses sind sicherlich die Forderung nach vollkommener Mobilität der Konsumenten und die alleinige Abhängigkeit der Wohnortentscheidung vom Angebot an lokalen öffentlichen Gütern (Annahme IV). Dabei ist das empische Problem zu lösen, ob öffentliche Güter, wie z.B. die Umweltqualität, tatsächlich relevant für die Wanderungsentscheidung von Individuen sind. Es gibt Anzeichen dafür, daß z.B. die Umweltqualität Grundstücks- und Hauspreise beeinflußt. In Los Angeles etwa sind die Grundstückspreise in Gegenden gesunken, in denen die Luftverschmutzung besonders stark ist, während in Regionen mit besserer Luftqualität die Preise gestiegen sind. Mobilität aufgrund unterschiedlicher. Angebote an lokalen öffentlichen Gütern scheint damit in der Realität beobachtbar zu sein. 2)

Mit Annahme III wird verlangt, daB ein Konsument aus einer sehr großen Anzahl von Gemeinden sein Steuer- und Güterpaket wählen kann. Daher können alle Konsumenten in Gemeinden ziehen, die das öffentliche Gut genau nach ihren Präferenzen anbieten, so daß im Gleichgewicht Pareto-Optimalität herrscht [ Vgl. Sandler, Tschirhart (1980, 1506-1508)]. Um eine realistischere Gemeindestruktur anzustreben, werden in der Litatur Modelle mit kleiner

1) Vgl. u.a. Pestieau $(1979,13-15)$

2) Die empirischen Aspekte, hier vor allem das eben angeschnittene Problem der Kapitalisierung, werden im folgenden nicht behandelt. Einen úberblick über die Literatur gibt Pestieau $(1979,3-11)$. 
Gemeindezahl diskutiert [Vgl.z.B. Stiglitz (1977), Flatters et al. (1974)].

Die Annahme, daß alle Konsumenten nur von Divideneneinkommen leben, liefert ein Mittel, das im Grunde genommen die Eigenheiten von Gemeinden aus dem Anpassungsmodell eliminiert. Wenn alle Einkommen aus Dividenden stammen, hängt die standortwahl eines Individuums nicht von der Allokation der Ressourcen im privaten Sektor der ökonomie ab. (Buchanan, Götz (1972)). Jedoch hängt die Wohnortentscheidung auch, und bestimmt in groBem Ausmaß, von der Allokation der Ressourcen im privaten Sektor ab. Aus diesen Gründen können die Annahmen (III) und (IV) nur im Rahmen der Clubtheorie aufrechterhalten werden (Vgl. Buchanan (1965)). Die Annahme (V), daß keine externen Effekte beim Angebot öffentlicher Güter entstehen, ist eine strenge Forderung. In der Literatur (Vgl. z.B. Breton (1970), Loehr (1974), Sandler/Shelton (1972), Sandler (1975)) wurde deshalb vielfach die Frage diskutiert, welche Auswirkungen "spill-overs" und "spill-ins" auf das Angebot öffentlicher Güter haben. Auf dieses Problem wird im folgenden nicht weiter eingegangen.

Durch die Annahmen (VI) und (VII) zusammen mit der Annahme (IV), ist praktisch das Phänomen des "crowding" als gelöst vorgegeben. Da dies aber für die Bestimmung der optimalen Gemeindegröße von ausschlaggebener Bedeutung ist, können diese Annahmen bei der Bestimmung des optimalen Angebots öffentlicher Güter nicht aufrecht erhalten werden (Vgl. Teil 2 und z.B. Flatters et al. (1974), Stiglitz (1977)).

Eine allgemeine Kritik an dem Tiebout-Konzept "voting with one's feet" soll hier noch kurz angesprochen werden. "3) Individuen können ihre präferenzen grundsätzlich auf zwei Arten ausdrücken, und zwar einmal über Eintritts- und Austrittsentscheidungen (z.B. beim Markt privater Güter) und das andere Mal über schriftliche oder verbale Kommunikation oder Stimmabgabe. Durch das TieboutKonzept wird jetzt praktisch der politische Willensbildungspro-

3) Vgl. Hirschmann (1970) oder Mueller (1979, Chapter 7). 
zeß durch die Entscheidung Emigration oder nicht ersetzt. Die Einwohner einer Gemeinde sind dann also nicht mehr bereit, ihre Forderungen an die Gemeinderegierung in einem langwierigen Prozeß durchzusetzen, sondern emigrieren einfach.

\section{Zur Charakterisierung von lokalen öffentlichen Gütern}

2. 1 Unterschiede zwischen reinen und lokalen öffentlichen Gütern

Die auf Samuelson (1954, 1969) zurückgehende Klassifizierung von Gütern in Private und Öffentliche bedient sich der Kriterien der "Ausschließbarkeit" und der "Rivalität des Konsums". Danach liegt ein öffentliches Gut vor, wenn es bei einer gegebenen Menge dieses Gutes nicht Inöglich ist, einzelne Individuen vom Konsum dieses Gutes auszuschließen, und bei steigender Zahl von Konsumenten die individuelle Bedürfnisbefriedigung irgend eines Individuums unbeeinträchtigt bleibt. Das Kriterium der "Rivalität des Konsums" spiegelt daher die in der angelsächsischen Literatur auch mit "CROWDING" oder "CONGESTION" bezeichneten Phänomene wider. Während das Ausschlußprinzip als "technologische" Eigenschaft eines Gutes unabhängig von der Anzahl der Konsumenten, deren Präferenzstruktur und der Produktionstechnologie einer ökonomie beschrieben werden kann, ergeben sich die als crowding bezeichneten Effekte entweder in Verbindung mit der Präferenzstruktur - im folgenden auch crowding im Konsumbereich genannt - oder in Verbindung mit der Produktionstechnologie - im folgenden mit crowding im Produktionsbereich bezeichnet. Lokale öffentliche Güter lassen sich als öffentliche Güter definieren, für welche eine Ausschlußtechnologie existiert. Ob von der Ausschlußtechnologie Gebrauch gemacht wird, also verschiedene Bezirke oder Gemeinden unterschiedliche lokale öffẹtliche Güterbündel anbiéten, ist von den jeweils vorliegenden, noch näher zu beschreibenden crowding-Effekten abhängig. Für den Fall, daß den lokalen öffentlichen Gütern keine crowding-Effekte zugeordnet sind, ist eine notwendige Bedingung für eine Pareteo-optimale Allokation die Nichtanwendung des Ausschlußprinzips. Um diese Behauptung zu 
überprüfen, sei von einer effizienten Zuordnung der Konsumenten auf verschiedene Bezirke oder Kommunen ausgegangen. Bezeichnet $y^{k}$ die Menge des öffentlichen Gutes, welches in Bezirk k produziert wird, so kann bei einer Ansiedlung aller Konsumenten in einem Bezirk neben der Menge privater Güter, die von einem Konsumenten in jedem beliebigen Bezirk konsumiert wird, zusätzlich der Betrag $\sum_{k=1}^{K} y^{k}$ an öffentlichen Gütern konsumiert werden. Da der Nutzenindex jedes Konsumenten bei gegebenem Konsum des privaten Gutes vom Konsum des öffentlichen Gutes abhängt und nicht von der Anzahl der "Mitkonsumenten", steigt durch ZusammenschluB aller Konsumenten zu einem Bezirk der Nutzenindex jedes Konsumenten.

Unter Beachtung der crowding-Effekte ergibt sich daher die Entscheidung über eine effiziente Anwendung der Ausschlußtechnologie oder die Bestimmung der optimalen Gemeindegröße als Lösung eines Optimierungsproblems. Bei diesem Optimierungsproblem werden die "Kosten des nicht angewandten Ausschlusses" in Form des crowding den "Kosten des Ausschlusses" gegenübergestellt. Im folgenden wird anhand eines einfachen 3-Gütermodelis das Problem der optimalen Gemeindegröße beschrieben.

Wir gehen von einer ökonomie aus, in der es zwei private Güter $Q$ und $w$ (Gut 2 und Gut 3) und ein lokales öffentliches Gut $y$ (Gut 1) gibt. Gut $3(w)$ ist ein Produktionsfaktor und stiftet beim Einsatz in Konsumprozessen keinerlei "Nutzen". Durch Einsatz des Gutes 3 wird das Gut 2 (Q) produziert, welches entweder direkt als Konsumgut $x$ nutzbar ist oder als einziger produktionsfaktor $z$ zur Herstellung des Gutes $1(y)$ dient. Die eben genannte Technologie der ökonomie wird beschrieben durch
(1) $Q=f(w)$
mit $f^{\prime}>0, f^{\prime \prime}=0, f(0)=0$
(2) $y=g(z, n)$
mit $g_{1}>0, g_{11} \leq 0, g_{2} \leqq 0$, $g(0, n)=0$ für beliebiges $n \in R_{+}$und $g(z, 0)>0$ für $z \in R_{+}$


Über das Produktionsverfahren $f$ kann mit der Inputmenge $w$ des Gutes 3 die Menge $Q$ des Gutes 2 produziert werden. Analog wird bei $\mathrm{n}$ Konsumenten mit $\mathrm{z}$, Einheiten des Gutes 2 über die Technologie g die Outputmenge $y$ an Gut 1 produziert. Die Abhängigkeit des Outputs an Gut 1 von der Zahl der Konsumenten wird CROWDING im Produktionsbereich genannt. Dieser crowding-Effekt läßt sich wie folgt interpretieren. Sei der Inputkombination $\left(z^{\prime}, n^{\prime}\right)$ der Output $Y^{\prime}$ zugeordnet. Um bei der konsumentenzahl $n^{\prime \prime}>n^{\prime}$ die Menge $y^{\prime} z u$ produzieren, ist der Faktoreinsatz $z^{\prime \prime}>z^{\prime}$ erforderlich. Dieser crowding-Effekt kann daher auch als die "Kosten des nicht angewandten Ausschlusses" interpretiert werden. Der crowding-Effekt im Produktionsbereich kann durch folgendes Beispiel verdeutlicht werden. Sei das öffentliche Gut ein Park, der von allen Einwohnern einer Gemeinde besucht werden kann. Sei y die Anzahl der Blumenbeete in diesem Park. Je nach Besucheranzahl $\mathrm{n}$ muß eine bestimmte Anzahl an Gärtnern dazu abgestellt werden, aufzupassen, damit niemand der Besucher die Blumenbeete zerstört. D.h. mit steigender Besucherzahl müssen immer mehr Gärtner als Aufpasser eingesetzt werden, so daß immer weniger Blumenbeete "produziert" werden können.

Die Präferenzen jedes Konsumenten $i$ seien durch folgende Nutzenfunktionen abbildbar

$$
\mathrm{u}^{\mathrm{i}}\left(\mathrm{x}^{\mathrm{i}}, \mathrm{y}^{\mathrm{i}}, \mathrm{n}\right) \text { mit } \mathrm{u}_{1}^{\mathrm{i}}, \mathrm{u}_{2}^{\mathrm{i}}>\mathrm{o}, \mathrm{u}_{3}^{\mathrm{i}} \leqq \mathrm{o}
$$

wobei $x^{i}\left(y^{i}\right)$ den Konsum des Individuums $i$ an Gut 2 (1) beschreibt. Der in (3) formulierte Einfluß der Bevölkerungsgröße (Gemeindegröße) auf den Nutzenindex des i wird CROWDING im Konsumbereich genannt. Ähnlich wie im produktionsbereich kann im Falle lokaler öffentlicher Güter ein derartiger EinfluB der Gemeindegröße à priori nicht ausgeschlossen werden. Im Unterschied zum crowding des produktionsbereiches kann bei der durch Beziehung (3) vorgenommenen Formulierung die Ursache des crowdingEffektes nicht eindeutig der Existenz des lokalen öffentlichen Gutes (Gut 1) zugeschrieben werden. Ein Beispiel für einen crowding-Effekt im Konsumbereich liefert Buchanan's (1965) Beispiel der Bildung eines Schwimmclubs. Der crowding-Effekt besteht hier- 
bei darin, daß die individuelle "Bedürfnisbefriedigung" bei steigender Zahl der Benutzer sinkt.

Geht man davon aus, daß alle Konsumenten über identische Anfangsausstattungen $w^{i}=1$ an Gut 3 verfügen, liegen erreichbare Allokationen bei der Bevölkerungszahl n vor, wenn die Beziehungen (1), (2) und

(4a) $\quad w=n$

(4b) $\quad Q=\sum_{i=1}^{n} x^{i}+z$

$$
y=y^{i}=y^{j} \text { für } i \neq j \text { und } i, j=1, \ldots, n
$$

erfüllt werden. Aus (5) ist erkennbar, daß zunächst von einem globalen öffentlichen Gut ausgegangen wird. Der lokale Charakter des öffentlichen Gutes wird an späterer Stelle präziser gefaßt.

Unterstellt man ferner, die produzenten der ökonomie verhalten sich als Mengenanpasser, die Gewinne werden über Gewinnanteilsrechte $\theta_{i}$ mit $\theta_{i}=\theta_{j}$ für $i \neq j$ vollständig auf die Konsumenten verteilt, die Kostenanteile zur Finanzierung des öffentlichen Gutes sind für alle Konsumenten gleich und alle Konsumenten verfügen über identische Nutzenfunktionen, können die gesamtwirtschaftlichen Implikationen alternativer Gemeindegrößen durch die nachstehenden Fallunterscheidungen verdeutlicht werden.

\section{2 Die gesamtwirtschaftlichen Implikationen} unterschiedlicher Gemeindegrößen

2.2.1 Optimale Gemeindegrößen bei crowding im Produktionsbereich Wir abstrahieren an dieser Stelle von crowding-Effekten im Konsumbereich, womit $u\left(x^{i}, y^{i}, n\right)=u\left(x^{i}, y^{i}, n^{\prime}\right)$ für beliebiges $n \neq n^{\prime}$ gilt. Da identische konsumenten vorliegen und der Finanzierungsanteil jedes Konsumenten am öffentlichen Gut $1 / n$ beträgt, folgt $x^{i}=x^{j}$ für $i \neq j$ und $i, j=1, \ldots, n$. Daher kann (4b) unter Berücksichtigung von $(4 a)^{\circ}$ und (1) geschrieben werden als $f(n)=n \cdot x^{i}+z$ bzw.

$$
x^{i}+\frac{1}{n} z=\frac{f(n)}{n}
$$


Die Beziehung (6) kann als Budgetrestriktion des Konsumenten i interpretiert werden, wobei $z^{i}=\frac{1}{n} z$ die "Opportunitäts"- Kosten des $i$ für das öffentliche Gut $y=g(z, n)$ bei der Bevölkerungsgröße $\mathrm{n}$ sind.

Fall A:

Crowding-Effekte bei der Produktion öffentlicher Güter

Sei $f(n)=n$ unterstellt. Unter dieser zusätzlichen Voraussetzung kann die Menge erreichbarer Allokationen für Konsument i durch das nachstehende Schaubild 1 dargestellt werden. Die im II. Quadranten des Schaubildes 1 eingezeichnete Gerade gibt die alternative Verwendung der Anfangsausstattung des Konsumenten i wider. Danach kann die Anfangsausstattung einerseits als Gut 2 konsumiert werden oder als Input $\mathrm{z}^{i}$ zur Produktion des Gutes 1 eingesetzt werden. $\mathrm{Da} f(n)=n$ unterstellt ist, ändert sich, bei Änderung

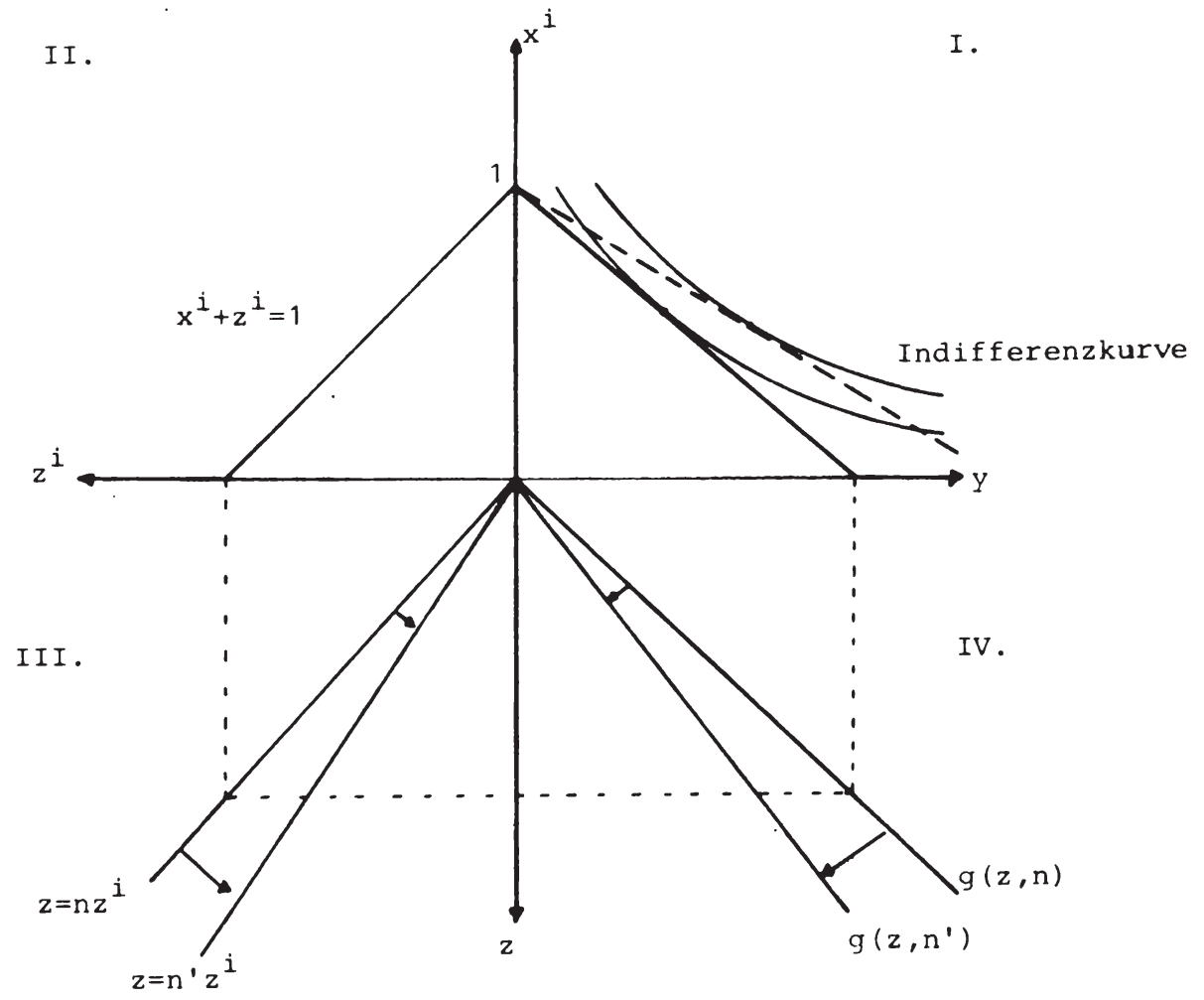

Schaubild 1 
der Bevölkerung $n$, die im II. Quadranten eingezeichnete Gerade nicht. Andererseits steigt bei steigendem $\mathrm{n}$ und konstantem $\mathrm{z}^{\mathrm{i}}$ der "gesamtwirtschaftliche" Input $z$ oder anders ausgedrückt der Finanzierungsanteil des $i$-ten Konsumenten $\frac{1}{n}$ sinkt bei steigendem $\mathrm{n}$. Dies ist in Schaubild 1 für $\mathrm{n}^{\prime}>\mathrm{n}$ durch Drehung der im III. Quadranten eingezeichneten Ursprungsgeradenverdeutlicht. Dieser Effekt kann als die Kosten der Anwendung des Ausschlußprinzips interpretiert werden. Diesem Effekt entgegengesetzt ist der im IV. Quadranten eingezeichnete crowding-Effekt bei der produktion des öffentlichen Gutes. Je nachdem, welcher der beiden Effekte dominiert, erhält man im I. Quadranten des Schaubildes 1 eine Drehung der dort eingezeichneten "individuellen Transformationskurve" um den Punkt $\mathrm{x}^{i}=1$.

Die eben genannten Implikationen steigender Konsumentenzahlen lassen sich bei crowding im produktionsbereich wie folgt algebraisch darstellen. Wegen der oben vorgenommenen Spezifikation gilt zunächst die oben deduzierte Gleichung (6), womit folgt

(6') $\quad z=\left(1-x^{i}\right) \cdot n$

Setzt man (6') in die Beziehung (2) ein, ergibt sich

$$
y=g\left(\left(1-x^{i}\right) \cdot n, n\right)
$$

und totale Differentiation von (7) nach $\mathrm{n}$ gibt

$$
\frac{d y}{d n} \mid \frac{d x^{i}}{d n}=0=g_{1} \frac{d z}{d n}+g_{2}=\underbrace{\left(1-x^{i}\right) g_{1}}_{1 . \text { Effekt }}+\underbrace{g_{2}}_{\text {2.Effekt }} \geqq 0
$$

Die Implikationen steigender Bevölkerung können im Fall des crowding im produktionsbereich durch die beiden in (8) formalisierten Effekte beschrieben werden. Steigt $n$ um eine Einheit, wandert also ein zusätzlicher konsument in unsere Region ein, dann steigt bei Konstanz des Inputs $z^{i}$ jedes Konsumenten der Gesamtinput um $\left(1-x^{i}\right)$ und ohne crowding im produktionsbereich ergäbe 
sich eine Verbesserung der Versorgung mit öffentlichen Gütern von $\left(1-x^{i}\right) g_{1}$. Dieser Effekt ist durch die bereits genannte Drehung der Kurve im III. Quadranten in Schaubild 1 skizziert. Diesem "reinen" öffentlichen Gutseffekt entgegengesetzt ist der "reine" crowding-Effekt $g_{2}<0$. Man erkennt, daß für $g_{2}=0-a l-$ so im Falle des reinen öffentlichen Guts - die im I. Quadranten des Schaubildes 1 eingezeichnete "individuelle Transformationskurve" für großes $n$ nahezu parallel zur $y$-Achse verläuft. Unter einer derartigen Konstellation konvergiert die optimale Gemeindegröße gegen unendlich. Geht man davon aus, daß bei kleinem $n$ der öffentliche Gutseffekt (Effekt 1) den crowding-Effekt dominiert und bei großem $n$ der crowding-Effekt dominiert, kann dies in Schaubild 1 verdeutlicht werden, indem zunächst für steigendes $n$ (und relativ kleines $n$ ) die im $I$. Quadranten gezeichnete "individuelle Transformationskurve" nach "außen" dreht und ab der optimalen Clubgröße für wachsendes $\mathrm{n}$ eine Drehung in umgekehrter Richtung stattfindet.

Ordnet man den im I. Quadranten des Schaubildes 1 eingezeichneten Tangentialpunkten von Indifferenzkurven und Budgetgeraden die jeweiligen Werte $u(x(n), y(n))=v(n) z u$, ist eine indirekte Nutzenfunktion $v$ definiert. Die indirekte Nutzenfunktion gibt den maximal erreichbaren Nutzenindex in Abhängigkeit von der Bevölkerungszahl n für einen Konsumenten an. Daher gilt

$$
v(n)=\max _{x, y} u(x, y) \text { u.d.B. } x+\frac{1}{n} z=\frac{f(n)}{n} \text { und } y=g(z, n)
$$

womit sich Schaubild 2 zeichnen läßt.

Fall B:

Crowding-Effekte bei der Produktion privater Güter

Im Gegensatz zum vorstehenden Abschnitt sei jetzt davon ausgegangen, daß $g(z, n)=z$ und $f^{\prime \prime}<0$ gilt. Dann kann die Beziehung (6) auch geschrieben werden als 


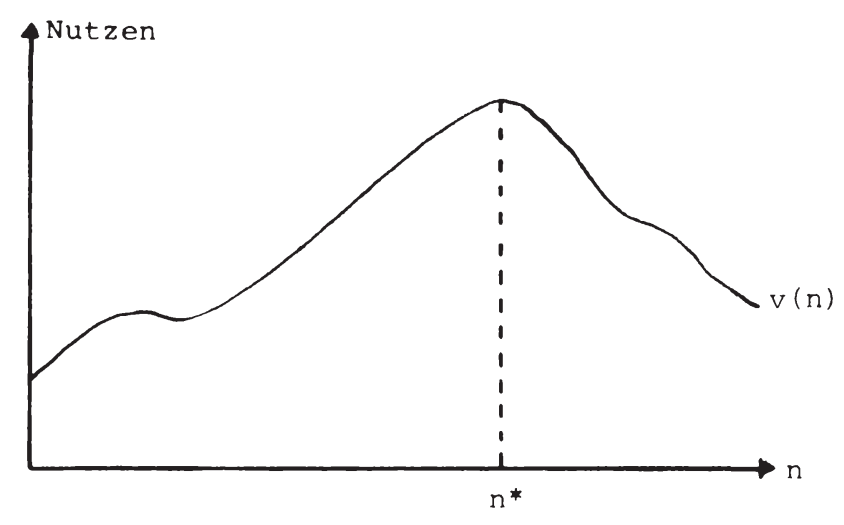

Schaubild 2

(6") $\quad x^{i}+\frac{1}{n} y=\frac{f(n)}{n}$

Analog zu Schaubild 1 können die jetzt auftretenden Effekte steigender Bevölkerungszahl durch das nachstehende Schaubild 3 verdeutlicht werden.

Da in diesem Falle sinkende Skalenerträge bei der produktion des Gutes 2 unterstellt sind, verschiebt sich bei steigendem $n$ die im II. Quadranten des Schaubildes 3 eingezeichnete Gerade parallel in Richtung Ursprung. Die Idee dieser Modellvariante geht auf Buchanan/Wagner (1970) zurück und diente Flatters et.al. (1974) und Stiglitz (1977) als Basismodell ihrer Analysen. Interpretiert wurde diese Modellvariante als "Ricardianisches Modell", indem die o.a. Autoren von einem lokal immobilen Produktionsfaktor "Land" ausgegangen sind und bei gegebener Landgrösse pro Gemeinde die Hypothese sinkender Grenzprodukte des Gutes 3 (Arbeit) bei steigender "Einwanderung" zugrunde gelegt wurde. Der im III. Quadranten des Schaubildes 3 verdeutlichte Effekt ist der bereits in Schaubild 1 angesprochene Kosteneffekt der Anwendung des Ausschlußprinzips. Das Zusammenwirken der beiden Effekte ist im I. Quadranten des Schaubildes 3 skizziert. Im Ge- 


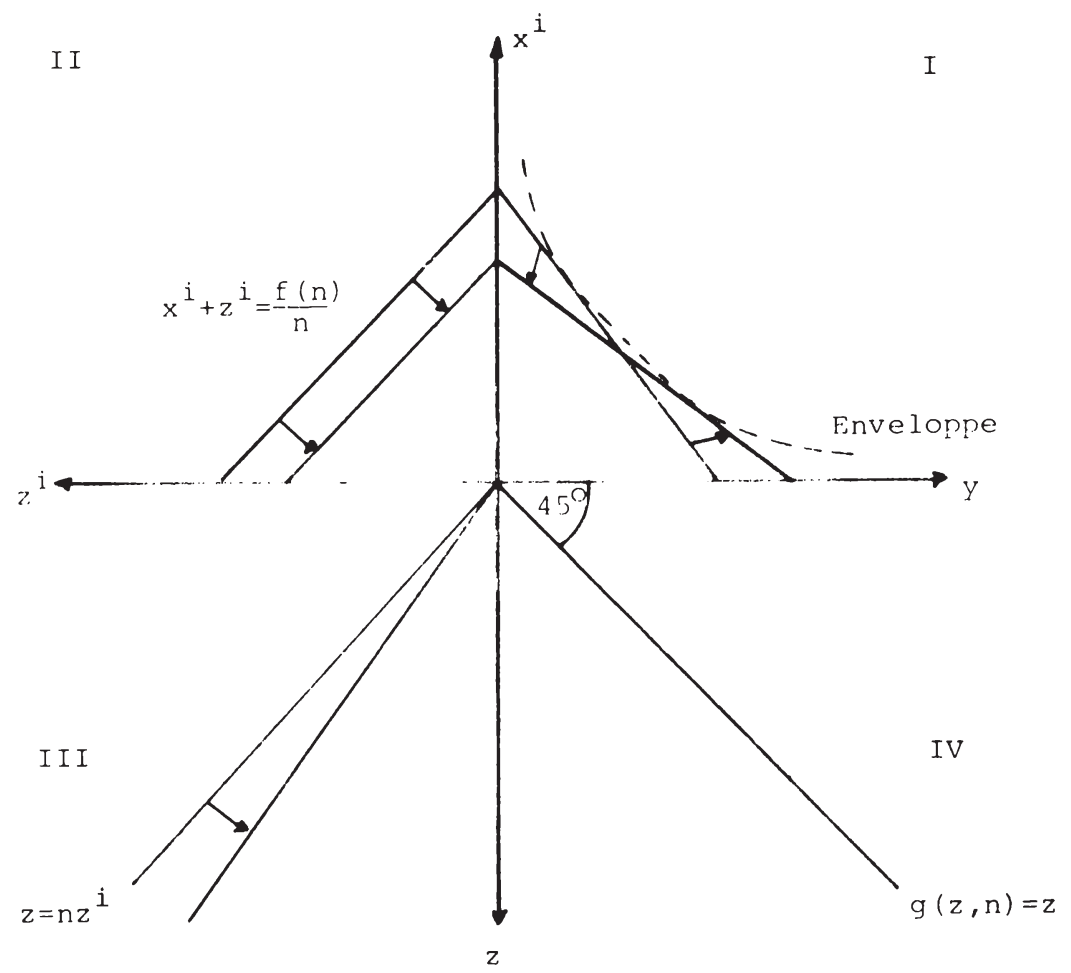

Schaubild 3

gensatz zu Schaubild 1 verschiebt sich bei variierendem $\mathrm{n}$ im obigen Fall zusätzlich der Ordinatenabschnitt der jeweils im I. Quadranten eingezeichneten Geraden. Zeichnet man im I. Quadranten des Schaubildes 3 für jedes $n \in R_{+}$die entsprechenden individuellen Transformationskurven ein, kann die Menge aller $\left(x^{i}, y\right)$-Kombinationen, die für unseren Einheitskonsumenten bei unterschiedlichen Gemeindegrößen erreichbar ist, als Fläche unter der gestrichelt gezeichneten Enveloppe in Schaubild 3 kenntlich gemacht werden. Damit wird besonders eindrucksvoll ein erstes Problem bei der Bestimmung der optimalen Gemeindegröße, nämlich die Nicht-Konvexität der Menge erreichbarer Allokationen 
bei alternativen Gruppengrößen, deutlich. ${ }^{4)}$ Aufgrund der NichtKonvexität der Menge erreichbarer Allokationen bei alternativen Gruppengrößen kann als Lösung der Optimierung einer Nutzenfunktion über der eben genannten Menge sowohl ein Maximum als auch ein Minimum resultieren. Dies sei anhand der nachstehenden Graphik verdeutlicht. ${ }^{5)}$

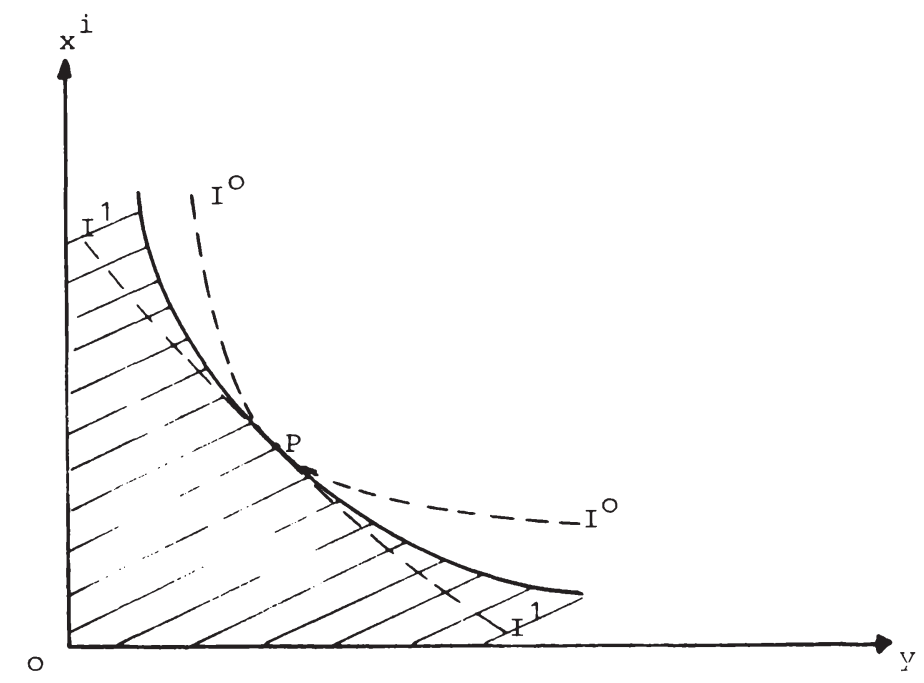

Schaubild 4

Die nicht-konvexe Menge erreichbarer Allokationen bei alternativen Gruppengrößen ist in Schaubild 4 schraffiert eingezeichnet. Die gestrichelt gezeichneten Kurven $\mathrm{I}^{\circ} \mathrm{PI}^{\circ}$ und $I^{1} \mathrm{PI}{ }^{1}$ geben jeweils eine Indifferenzkurve aus zwei verschiedenen Präferenzsystemen wider. Die Präferenzsysteme werden dabei so gewählt, daß sich die Indifferenzkurven im Punkt $P$ tangieren. Die Präferenzstrukturen unterscheiden sich dadurch voneinander, daß einmal

4) Vgl. hierzu Stiglitz (1977) und besonders die von Milleron (1977) bei der Diskussion des Stiglitz-Beitrags dargelegten Argumente.

5) Vgl. hierzu auch Stiglitz (1977, 277 ff.). 
eine relativ große Komplementarität zwischen privatem und öffentlichem Gut unterstellt wurde, $\mathrm{I}^{\circ} \mathrm{PI}^{\circ}$, und im zweiten Beispiel eine relativ große substitutionselastizität zwischen privatem und öffentlichem Gut vorliegt. Der Punkt $P$ ist weiterhin ein Punkt auf einer nicht eingezeichneten individuellen Transformationskurve bei einer Bevölkerungszahl n* (analog zu Schaubild 3). Aus Schaubild 4 wird deutlich, daß die indirekte Nutzenfunktion $v(n)$ im Falle der durch $I^{\circ} I^{\circ}$ gekennzeichneten Indifferenzkurve bei der Bevölkerungszahl $n^{*}$ ihr Maximum annimmt. Ist $I^{1} P I^{1}$ eine Indifferenzkurve unseres Einheitskonsumenten, ist bei der Bevölkerungszahl $n^{*}$ ein Minimum der indirekten Nutzenfunktion gegeben. Zahlenbeispiele für jeden der beiden Fälle werden im Anhang diskutiert.

\subsubsection{Optimale Gemeindegrößen bei crowding im Konsumbereich}

Um die "reinen" crowding-Effekte im Konsumbereich isolieren zu können, abstrahieren wir an dieser Stelle von den crowding-Effekten im Produktionsbereich. Deshalb wird $f(n)=n$ und $g(z, n)=z$ unterstellt. Die Implikationen steigender Konsumentenzahlen lassen sich dann folgendermaßen algebraisch darstellen. Da $f(n)=n$ unterstellt wird, kann die Gleichung (6) geschrieben werden als

$$
\text { (6"') } \quad x^{i}=1-\frac{1}{n} z
$$

Setzt man (6"') in (3) und beachtet $y^{i}=g(z, n)$ für $i=1, \ldots, n$ und $g(z, n)=z$ erhält man $u\left(1-\frac{1}{n} z, z, n\right)$. Der Einfluß eines Einwanderers auf das Nutzenniveau des $i$ ergibt sich dann als

$$
\frac{\mathrm{du}}{\mathrm{dn}}=\mathrm{u}_{1} \frac{\mathrm{dx}}{\mathrm{dn}}+\mathrm{u}_{2} \frac{\mathrm{dz}}{\mathrm{dn}}+\mathrm{u}_{3}
$$

mit

$$
\begin{aligned}
& \frac{d x^{i}}{d n}=\frac{z}{n} 2-\frac{1}{n} \frac{d z}{d n} \quad \text { Daher gilt } \\
& \frac{d u}{d n}=u_{1} \frac{z}{n} 2+u_{3}+\left(u_{2}-\frac{1}{n} u_{1}\right) \frac{d z}{d n}
\end{aligned}
$$

Geht man davon aus, daß ein optimaler "Angebots-Mix" zwischen öffentlichem und privatem Gut angeboten wird, dann gilt die Samuelson-Bedingung, daß die Grenzrate der Transformation gleich 
der Summe der Grenzraten der Substitution zwischen öffentlichem und privatem Gut ist (d.h. $\mathrm{n} \frac{\mathrm{u}_{2}}{\mathrm{u}_{1}}=1$ ), so daß

$$
\frac{\mathrm{du}}{\mathrm{dn}}=\mathrm{u}_{1} \frac{\mathrm{z}}{\mathrm{n}} 2+\mathrm{u}_{3}=\mathrm{u}_{2} \frac{\mathrm{z}}{\mathrm{n}}+\mathrm{u}_{3} \geqq 0
$$

Erneut liegen zwei Effekte vor, die einander entgegengesetzte Wirkung haben, nämlich

1. $u_{1} \frac{z}{n^{2}}\left(u_{2} \frac{z}{n}\right)>0:$ Nutzenzuwachs durch vermehrte Produktion bei steigendem $n$

2. $u_{3} \leqq 0:$ Nutzenminderung durch crowding im Konsumbereich.

Bei der graphischen Verdeutlichung der crowding-Effekte im Konsumbereich kann wie folgt argumentiert werden. Zunächst liegt von der Produktionsseite des Modells der jeweils im III. Quadranten der Schaubilder 1 und 3 angedeutete Kosteneffekt der Anwendung des Ausschlusses vor. Die individuelle Transformationskurve wird deshalb für alternatives $n$ durch die im II. Quadranten des nachstehenden Schaubildes 5 eingezeichneten Geraden verdeutlicht.

Um die crowding-Effekte im Konsumbereich auf einfache Weise in Schaubild 5 integrieren zu können, sei von einer separablen Nutzenindexfunktion $\operatorname{der}$ Form $U=\min \left(x^{i}, y\right)-n$ ausgegangen. Das einer Güterkombination $\left(x^{i}, y\right)$ zuordenbare Nutzenniveau läßt sich dann ohne Berücksichtigung der crowding-Effekte im Konsumbereich anhand des im II. Quadranten des Schaubildes 5 eingezeichneten Expansionspfades verdeutlichen. Da die Güter $\left(x^{i}, y\right)$ streng komplementär im Konsum sind - angedeutet über die gestrichelt gezeichneten Indifferenzkurven - impliziert die, über die Erhöhung der Konsumentenzahl ausgelöste, "Preissenkung" des öffentlichen Gutes lediglich einen Einkommenseffekt (der Substitutionseffekt ist null). Die "gleichgewichtige" Allokation zwischen privatem und öffentlichem Gut ist daher für alternatives $n$ durch die Schnittpunkte von "individueller Transformationskurve" und Expansionspfad gegeben. Ohne Berücksichtigung des crowdingEffekts können daher die erreichbaren individuellen Nutzenniveaus bei alternativer Bevölkerungszahl über die im I. Quadranten des 


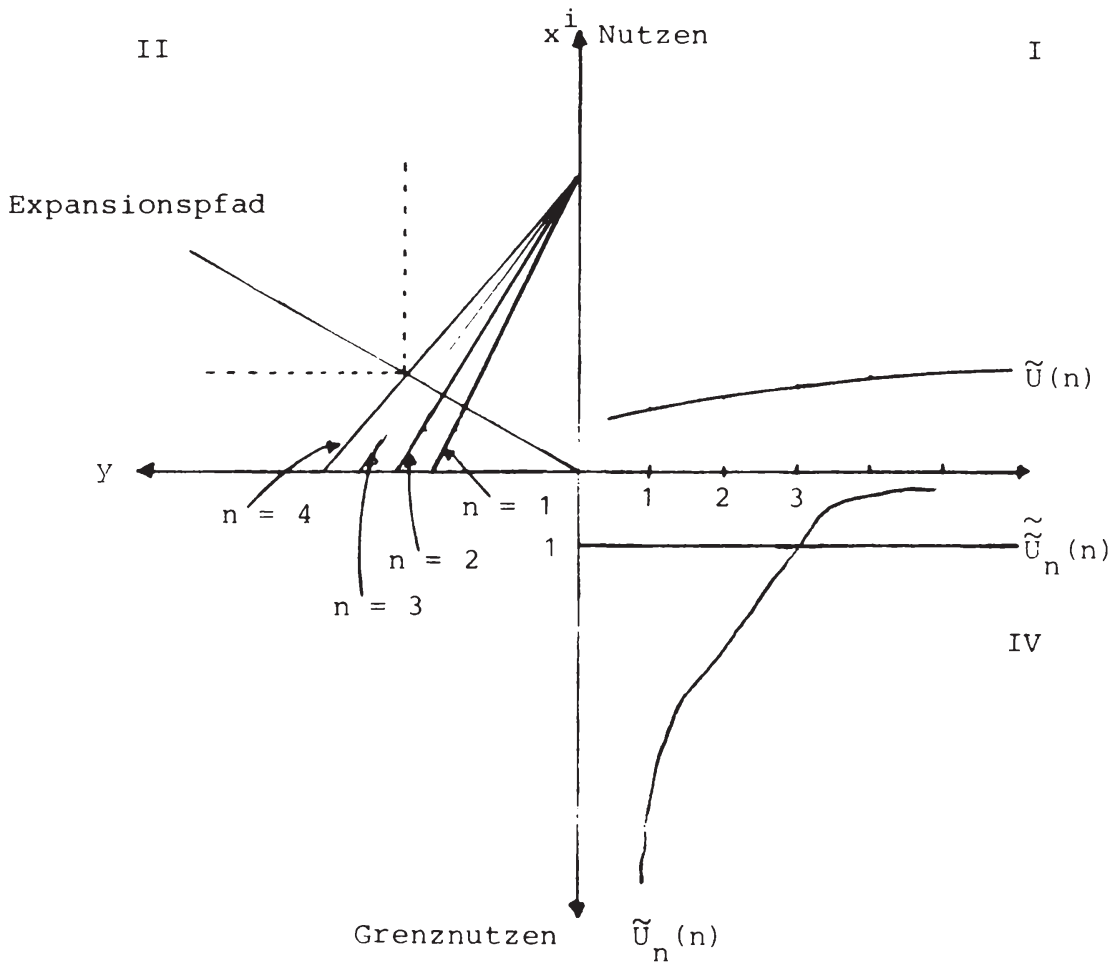

Schaubild 5

Schaubildes 5 "hergeleitete" Nutzenfunktion $\widetilde{U}(n)=\min \left(x^{i}(n)\right.$, $y(n))$ veranschaulicht werden. Der "Grenzschaden des Ausschlusses eines zusätzlichen Konsumenten" ist im IV. Quadranten als Ableitung der konkaven Funktion $\widetilde{U}$ nach $n$ eingezeichnet. Die im vorliegenden Beispiel unterstellten crowding-Effekte erhält man als $\tilde{U}(n)=n$, womit der "Grenznutzen des Ausschlusses eines zusätzlichen Konsumenten" $\tilde{U}_{n}(n)=1$ ist. Demnach ist aus dem IV. Quadranten des Schaubildes 5 die optimale Gemeindegröße als Schnittpunkt der beiden "Grenznutzenfunktionen" erkennbar. 
3. Dezentrale Allokationskonzepte und das Problem der optimalen Gemeindegröße

Ein speziell mit lokalen öffentlichen Gütern zusammenhängendes Problem ist das problem der optimalen Gemeindegröße. Die Effizienz eines dezentralen Allokationsmechanismus wie etwa des "voting with one's feet" Mechanismus ist davon abhängig, inwieweit sich ein derartiger Mechanismus zur Lösung des Problems der optimalen Gemeindegröße eignet. Im folgenden wird der Ausdruck "voting with one's feet" Mechanismus präzisiert, indem eine Palette möglicher Tiebout-Konzepte vorgestellt wird, wobei die einzelnen Gleichgewichtskonzepte differenziert werden können

(a) nach dem Informationsgrad der dezentralen Entscheidungseinheiten

- Konzept $1,2 \mathrm{a}, 2 \mathrm{~b}$ gegenüber 3 -

(b) nach dem social choice Mechanismus innerhalb einzelner Bezirke

- Bezirke als Produktionsunternehmen versus Mehrheitswahl als innerregionales Koordinationsinstrument -

(c) nach der Finanzierungsart der lokalen öffentlichen

Güter innerhalb der Bezirke

- Kopfsteuer, Einkommensteuer -.

Untersucht werden.Eigenschaften - speziell die Optimalität dieser Konzepte oder anders formuliert, die Frage, inwieweit durch Konsumentenmobilität ein "marktmässiges" Äquivalent für eine ökonomie mit lokalen öffentlichen Gütern konstruierbar ist. Zum Zwecke einer anschaulicheren Darstellung wird dabei das bisher verwendete Modell durch die nachstehenden Prämissen modifiziert. Wir gehen von einer ökonomie mit $\mathrm{N}$ Konsumenten aus. Diese $\mathrm{N}$ Konsumenten lassen sich in unterschiedliche Typen einteilen, wobei Konsumenten des gleichen Typs über gleiche Nutzenfunktionen und gleiche Anfangsausstattungen verfügen. Weiterhin sei unterstellt, daß es Konsumenten möglich ist, sich zu Gemeinden oder Bezirken zusammenzuschließen. Eine Zerlegung der Menge aller Konsumenten 
der ökonomie in $\mathrm{k}=1, \ldots, \mathrm{K}$ Bezirke oder Gemeinden wird auch eine Gemeinde- oder Bezirksstruktur genannt und durch $J(K) \equiv\left\{J_{1}, \ldots, J_{K}\right\}$ beschrieben, wobei $\left|J_{K}\right|=n_{k}$ die Anzahl der in einem Bezirk lebenden Konsumenten angibt. 6) 7) ${ }^{\mathrm{k}}$ In der Ökonomie gibt es zwei Arten von Gütern, und zwar lokale öffentliche und private. Bezüglich der Produktionsfunktionen $f, g$ wird unterstellt

(a) $f(w)=w$, womit konstante skalenerträge vorliegen und die gleichgewichtigen Preise zwischen den beiden privaten Gütern gleich sind. Im folgenden wird daher nicht mehr zwischen diesen Gütern differenziert, womit unser Modell auf ein 2-Gütermodell "schrumpft".

(b) $g(z, n)=h(n) \cdot z$, d.h. bei der Produktion des lokalen öffentlichen Gutes liegen bei gegebener Gruppengröße $n$ ebenfalls konstante Skalenerträge vor. Diesem "Verlust" an Allgemeinheit steht eine Verringerung des formalen Modellaufbaues in Form der hierdurch möglichen nicht expliziten Berücksichtigung von Gewinnanteilsrechten entgegen.

Eine Allokation der Ökonomie ist ein Tripel $\left(J(K),\left(y^{k}, z^{k}\right)\right.$, $\left.\left(x^{i}, y^{i}\right)\right)$, wobei $J(K)$ eine Bezirksstruktur, $\left(y^{k}, z^{k}\right)$ eine Produktion für $k=1, \ldots, k$ ist und $\left(x^{i}, y^{i}\right)$ ein Konsum für $i=1, \ldots, N$ ist.

Definition 1:

Eine Allokation $\left(\mathrm{J}(\mathrm{K}),\left(\mathrm{y}^{\mathrm{k}}, \mathrm{z}^{\mathrm{k}}\right),\left(\mathrm{x}^{\mathrm{i}}, \mathrm{y}^{\mathrm{i}}\right)\right)$ ist erreichbar, wenn gilt

$$
y^{k}=h_{k}\left(n_{k}\right) \cdot z^{k} \quad \text { für } k=1, \ldots, k
$$

(ii) $\quad\left(x^{i}, y^{i}\right) \in R_{+}^{2}$ für $i=1, \ldots, N$

$$
\sum_{i=1}^{N} x^{i}+\sum_{k \in J_{k}} z^{k}=\sum_{i=1}^{N} w^{i}
$$

(iv) $\quad i, j \in J_{k}$ und $i \neq j$ impliziert $y^{i}=y^{j}=y^{k}$ für $k=1, \ldots, k$

6) Im folgenden werden die Ausdrücke Gemeinde, Bezirk, Region aber auch Wortzusammensetzungen wie etwa Bezirksstruktur, Gemeindestruktur jeweils synonym gebraucht.

7) Da eine Bezirksstruktur $J(K)$ eine Zerlegung der Menge der Konsumenten ist, ist per Definition ausgeschlossen, daß einzelne Konsumenten"Wohnsitze" in mehreren Gemeinden haben. Diese Forderung wird in Buchanans "Club-Theorie" nicht erhoben. 
Durch Bedingung (i) der Definition 1 kommt zum Ausdruck, daß die Produktion in Bezirk $k$ unter der Produktionstechnologie dieses Bezirkes "herstellbar" ist. Gemäß Bedingung (iv) der Definition 1 wird ein lokales öffentliches Gut dadurch definiert, daß für dieses Gut eine Ausschlußtechnologie derart existiert, daß alle Konsumenten, die nicht in einem bestimmten Bezirk wohnen, vom Konsum des innerhalb dieses Bezirks angebotenen öffentlichen Gutes ausgeschlossen werden.

Nachdem gesagt wurde, was unter erreichbaren Allokationen in der vorstehenden ökonomie mit lokalen öffentlichen Gütern zu verstehen ist, sollen alternative Gleichgewichtskonzepte einer derartigen ökonomie definiert und diskutiert werden. Die Gemeinsamkeiten dieser Gleichgewichtskonzepte liegen darin, daß private Güter über Wettbewerbsmärkte allokiert werden und die Allokation der lokalen öffentlichen Güter in jedem Bezirk eine Bezirksregierung koordiniert. $\mathrm{Zu}$ diesem Zwecke erhebt die Regierung jedes Bezirks eine steuer. Das Steueraufkommen einer Region wird ausschließlich zur Finanzierung der lokalen öffentlichen Güter in dieser Region verwendet. Durch seine Mobilität hat daher ein Konsument die Möglichkeit, zwischen $\mathrm{k}=1, \ldots, \mathrm{k}$ Angeboten an öffentlichen Gütern und den zugeordneten Steuer zu wählen.

\section{1 Gemeinden als gewinnmaximierende produzenten}

Definition 2:

Eine Allokation $\left(J(K),\left(y^{k}, z^{k}\right),\left(x^{i}, y^{i}\right)\right)$ und ein Preissystem $p \equiv\left(p^{0}, p^{1}, p^{2}, \ldots, p^{K}\right)$ ist ein Gleichgewicht nach Konzept (1), wenn gilt

(i) die Allokation ist erreichbar

(ii) Für $\left(\mathrm{y}^{\mathrm{k}}, \mathrm{z}^{\mathrm{k}}\right)$ mit $\mathrm{k}=1, \ldots, \mathrm{k}$ gilt.

$$
p^{k}{ }^{k}-p^{o} z^{k} \geqq p^{k} \tilde{y}^{k}-p^{\sigma}{ }^{k} \text { mit } \tilde{y}^{k}=h_{k}\left(n_{k}\right) \sim_{z}^{k}
$$

(iii) Für $i=1, \ldots, N$ gilt, daß $i \in J_{k}$ impliziert

$$
\begin{aligned}
& p^{o} x^{i}+\frac{p^{k}}{n_{k}} y^{k}=e^{i} \text { und } u^{i}\left(x^{i}, y^{k}, n_{k}\right) \geqq u^{i}\left(\tilde{x}^{i}, y^{k}, n_{k}\right)
\end{aligned}
$$

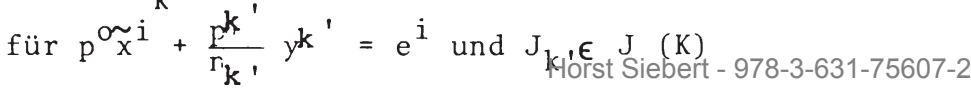


Mit $\mathrm{p}^{\mathrm{O}}$ wir der Preis des Gutes 1 bezeichnet und $\mathrm{p}^{\mathrm{k}}$ bezeichnet den Preis des öffentlichen Gutes in Region $k=1, \ldots, \mathrm{K}$. Die Bedungung (ii) der Definition 2 besagt, daß beim preissystem $p$ der Produktionsplan "jedes Bezirks" gewinnmaximal bezüglich der Technologie dieses Bezirkes ist. Nach dem oben Gesagten kann diese Bedingung auch dahingehend interpretiert werden, da $\beta$ die Bezirksregierung bei diesem Konzept lediglich aus einer "technischen" Abteilung besteht, deren einzige Aufgabe darin besteht, bei Mengenanpassung eine gewinnmaximale produktion anzubieten. Über die Bedingung (iii) der obigen Definition wird gefordert, daß beim Preissystem $p$ für einen Konsumenten der Gemeinde k das dort vorliegende Angebot $y^{k}$ und damit die dem Einkommen $e^{i}=p^{0} w^{i}$ zugeordente Menge an privatem Gut $x^{i}$ "besser" ist, als das entsprechende Angebot $y^{k^{\prime}}$ einer Gemeinde $k^{\prime}$; Dabei ergäbe sich unter Berücksichtigung der steuerzahlung $\frac{\mathrm{p}^{\mathrm{k}^{\prime}}}{\mathrm{n}_{\mathrm{k}^{\prime}}} \mathrm{y}^{\mathrm{k}^{\prime}}$ in Bezirk $\mathrm{k}^{\prime}$ für diesen Konsumenten ein privater Güterkonsum in Höhe von $\widetilde{x}^{i}$. Daher impliziert diese Bedingung, daß in einem Gleichgewicht nach Konzept (1) kein Konsument mehr wandert. Interessant an der Bedingung (iii) ist das Ausma $\beta$ an Informationen oder die Informationsmenge, die einem Konsument bei einer Emigrationsentscheidung zur Verfügung steht. Der Konsument kennt bei diesem Konzept die steuersätze $\frac{\mathrm{pk}^{\prime}}{\mathrm{n}_{\mathrm{k}^{\prime}}}$ und Angebote $\mathrm{y}^{\mathrm{k}^{\prime}}$ in der bestehenden Bezirksstruktur, also direkte oder "am Markt" beobachtbare Informationen. Diese Informationen gehen als Daten in sein Emigrationskalkül ein, womit eventuell entstehende externe Effekte eines Regionenwechsels nicht in Erwägung gezogen werden. Die Schwächen eines derartigen, auf ein institutionelles und informationsmäßiges Minimum beschränktes Tiebout-Konzept, lassen sich anhand des nachstehenden Beispiels erkennen. Wir spezifizieren die vorstehende ökonomie dadurch, daß identische Konsumenten, kein crowding im Konsumbereich und die Funktion $h_{k}\left(n_{k}\right)=\frac{1}{n_{k}}$ angenommen wird. Da - wie bereits betont die einzige Aufgabe der "technischen Abteilung" der Bezirksregierung darin besteht, eine gewinnmaximale produktion anzubieten, bezeichnet der Punkt $P$ in Schaubild 6 eine gleichgewichtige Allokation gemäB Konzept 1, für den Fall identischer Produktionspläne in allen Bezirken. 


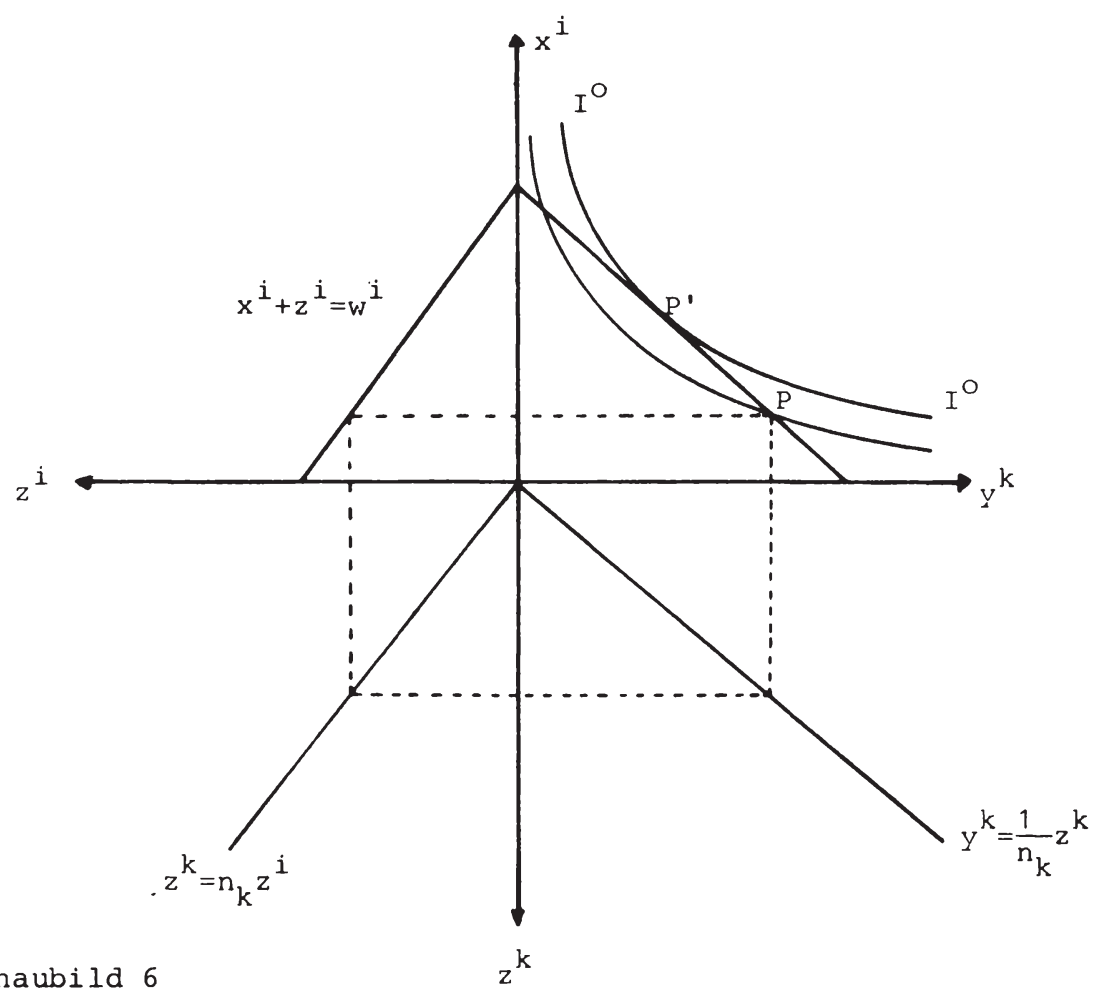

Schaubild 6

Aufgrund der eben spezifizierten Produktionsfunktion kann wegen Beziehung (8) die Budgetgerade jedes Konsumenten bei beliebiger Bezirksstruktur durch die Gerade mit den Punkten $P$ und $P^{\prime}$ gezeichnet werden. Bietet dann jeder Bezirk die im Punkt $P$ zugeordnete Menge an öffentlichen Gütern an, wird die entscheidende Schwäche des Konzepts (1), nämlich die mangelhafte Rückkopplung zwischen der "technischen Abteilung" der Bezirksregierung und dem Konsumenten deutlich.

\subsection{Innerregionale Koordination durch Mehrheitswahl}

Das eben beschriebene Defizit an innerregionaler Koordination kann bei gleicher Informationsmenge der dezentralen Entscheidungseinheiten dadurch abgebaut werden, daß ein zusätzlicher innerregionaler Koordinationsmechanismus in unser Modell eingebaut 
wird. Ein in der Literatur der lokalen öffentlichen Güter häufig verwendetes innerregionales Koordinationsinstrument ist die Mehrheitswahl. 8) Im folgenden wird die Erweiterung des Gleichgewichtskonzepts (1) durch Einbindung eines Mehrheitswahlprozesses unter Berücksichtigung alternativer Finanzierungsformen des öffentlichen Gutes - nämlich der Kopfsteuer in Definition 3a und der proportionalen Einkommensteuer in Definition 3b - dargestellt.

Definition 3a:

Eine Allokation $\left(J(K),\left(y^{k}, z^{k}\right),\left(x^{i}, y^{i}\right)\right)$ und ein Preissystem $p$ ist ein Gleichgewicht nach Konzept(2.a) der vorstehenden ökonomie, wenn neben den Bedingungen(i),(ii) und (iii) der Definition 2 zusätzlich gilt

(iv) $y^{\mathrm{k}}$ ist ein "Mehrheitswahl-Gemeinde-Gleichgewicht" für jedes $J_{k} \in J(K)$.

Über die Bedingung (iv) wird gefordert, daß in jeder Region das Angebot $\mathrm{y}^{\mathrm{k}}$ an Gut 2 von jeweils mindestens $50 \%$ aller Konsumenten dieser Region im direkten Vergleich mit jeder anderen Alternative der Vorzug gegeben wird. Das Entscheidungskalkül eines Konsumenten bei der Wahl in der Gemeinde läßt sich dabei durch die folgende Überlegung beschreiben. Beim Preissystem p stellt sich für einen Konsumenten innerhalb einer Region das Optimierungsproblem

$\max _{x^{i}, y^{i}} u^{i}\left(x^{i}, y^{i}, n_{k}\right)$ u.d.B. $p^{\circ} x^{i}+\frac{1}{n_{k}} p^{k} y^{i}=e^{i}$ für i $\varepsilon J_{k}$

Gemäß diesem Kalkül ist es einem Konsumenten möglich, beim Preissystem $p$ und der Bezirksgröße $n_{k}$ jeder Menge $y^{i}$ einen wert (Nutzen) $\mathrm{v}^{i}\left(\mathrm{y}^{i}\right)$ zuzuordnen. Dies läßt sich anhand des nachstehenden Schaubildes 7 verdeutlichen.

Für die in Definition 3a beschriebene gleichgewichtige Menge $y^{k}$ des lokalen öffentlichen Gutes wird dann gefordert, daß für

8) Vgl. hierzu u.a. Stiglitz (1977), Westhoff (1977). 


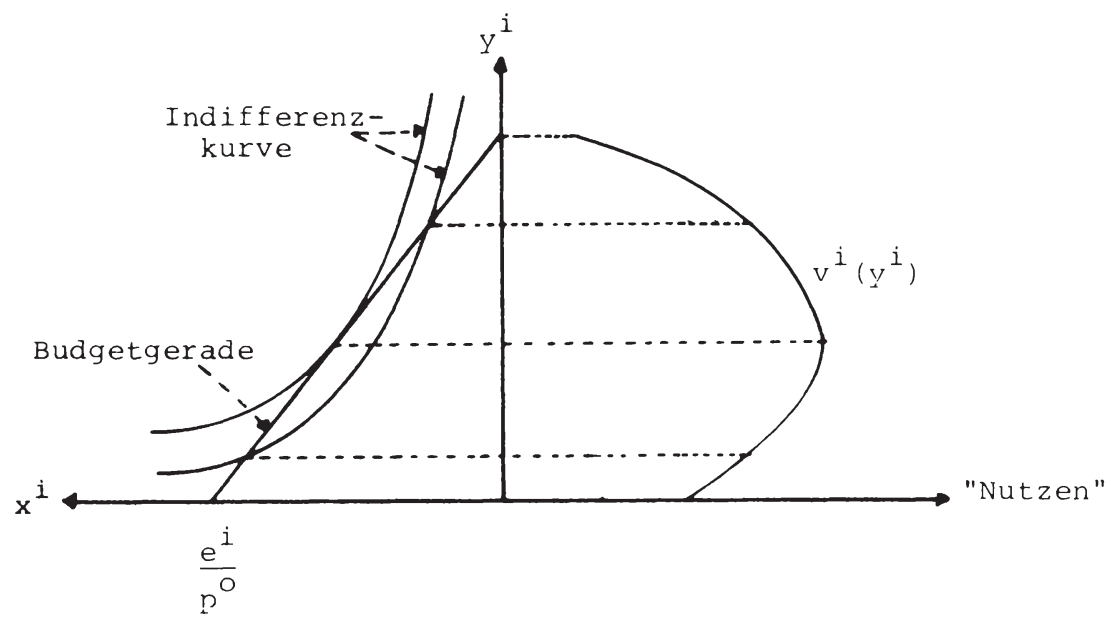

Schaubild 7

beliebiges Angebot $y^{i}$ gilt

$$
\left|\left\{\mathrm{i} \in J_{K}: v^{i}\left(y^{k}\right) \geqq v^{i}\left(y^{i}\right)\right\}\right| \geqq \frac{n_{k}}{2}
$$

wobei |A| die Mächtigkeit einer Menge A bezeichnet. Zur besseren Veranschaulichung des Gleichgewichtskonzepts (2a) sei ein zweistufiger Tâtonnement-Prozeß an ein derartiges Gleichgewicht skizziert.

Auf Stufe 1 des Tâtonnement-Prozesses kommuniziert bei gegebener Gemeindegröße (keine Mobilität) ein Auktionator mit der "technischen Abteilung" der Bezirksregierung und dem Mediankonsument so lange, bis ein preissystem $p$ gefunden ist, bei welchem die Nachfrage des Mediankonsumenten mit dem Angebot der "technischen Abteilung", bei ausgeglichenem Budget der einzelnen Regionen, ubereinstimmt. Ist ein derartiger zustand auf stufe 1 des prozesses erreicht, beseitigt der Auktionator sämtliche Mobilitätsbeschränkungen zwischen den $\mathrm{k}=1, \ldots, \mathrm{K}$ Bezirken. Unter dieser Voraussetzung überprüft dann auf stufe 2 des Prozesses jeder Konsument die Gültigkeit des in Bedingung (iii) der Definition 2 defi- 
nierten Kalküls. Stellen dann einige Konsumenten durch Vergleich der Steuern und der öffentlichen Güterbündel der verschiedenen Regionen fest, daß sie durch Emigration einen höheren Nutzenindex realisieren können, wandern sie in die entsprechenden Gemeinden. Kommt es dabei zu "Nettowanderungen" zwischen den Regionen, werden die auf Stufe 1 etablierten Gemeindestrukturen gestört. Daher verordnet der Auktionator erneut Mobilitätsbeschränkungen und der Proze $\beta$ setzt sich wie zuvor beschrieben fort.

Bei näherer Inspektion des Gleichgewichtskonzept (2a) stellt man fest, daß die im Beispiel des Schaubildes 6 skizzierten Probleme der innerregionalen Koordination durch die Einbettung der Mehrheitswahl gelöst wurden. Da die Anteile jedes Konsumenten einer Region an der Finanzierung des lokalen öffentlichen Gutes gleich sind, gilt $T^{i}=T^{j}=\frac{1}{n_{k}} p^{k} y^{k}$ für $i, j \in J_{k}$, womit eine Kopfsteuer gegeben ist. Diese Gleichheit der Finanzierungsanteile für alle Konsumenten in einem Bezirk ist bei einer proportionalen Einkommensteuer in der Regel nicht mehr gewährleistet. Da in der Realität ein Großteil der öffentlichen Ausgaben über Einkommensbesteuerung finanziert werden, scheint es nicht uninteressant, die Implikationen regionalisierter Einkommensteuern zu untersuchen. In der nachstehenden Definition $3 b$ wird daher ein Tiebout-Konzept definiert, welches sich vom Konzept (2a) nur dadurch unterscheidet, daB die Kopfsteuer durch eine proportionale Einkommensteuer ersetzt wird.

Definition $3 \mathrm{~b}$ :

Eine Allokation $\left(J(K),\left(y^{k}, z^{k}\right),\left(x^{i}, y^{i}\right)\right)$ und ein Preissystem $p$ ist ein Gleichgewicht nach Konzept (2b), wenn die Bedingungen (i), (ii) der Definition $3 a$ erfüllt werden und die den Bedingungen (iii) und (iv) zugrundeliegenden Entscheidungskalküle dadurch modifiziert werden, daß in den Budgetrestriktionen

$$
\frac{1}{n_{k}} \operatorname{durch~} e^{i}\left(\sum_{i \in J_{k}} e^{i}\right)^{-1} \quad \text { ersetzt wird. }
$$

Setzt man dann $p^{k} y^{k}\left(\sum_{i \in J_{k}} e^{i}\right)^{-1}=t^{k}$, kann die Budgetrestriktion 
auch geschrieben werden als $\mathrm{p}^{\circ} \mathrm{x}^{i}+t^{k} e^{i}=e^{i}$ und $t^{k}$ läßt sich als der proportionale Einkommensteuersatz der Region k erklären. Die Kalküle der dezentralen Entscheidungseinheiten können dann bei der Einkommensbesteuerung analog zu denen der Kopfsteuer - bei den Konsumenten also durch Schaubild 7 - beschrieben werden. Bei der Wahl in jeder Region bestimmt dann jeder Konsument einer Region, bei gegebenem Einkommen, gegebenem Preissystem und gegebener Einkommensteuer seine beste Menge an dem öffentlichen Gut. 9) 10)

9) Eine andere Variante der Definition der Entscheidungskalküle der Konsumenten bei Einkommensbesteuerung schlägt Westhoff (1977) vor. Bei Westhoff liegt im Gegensatz zum obigen Kalkül bei der Mehrheitswahl eine zwei-dimensionale Alternativenselektion vor. Jeder Konsument einer Region schreibt gemäß dem Westhoff'schen Kalkül beim Abstimmungsvorgang ein Tupel $(t, y)$ aus Einkommensteuersatz und öffentlichem Gut auf seinen Stimmzettel. $\mathrm{Zu}$ diesem Zwecke definiert Westhoff (1977) eine indirekte Nutzenfunktion über dem $(t, y)$-Raum. Unter Beachtung der Budgetrestriktion des Bezirkes gibt dann jeder Konsument bei der Wahl seine beste $(t, y)$-Kombination an. Dies kann graphisch veranschaulicht werden durch folgendes Diagramm (vgl.Westhoff, 1977 , S. 87 f.)

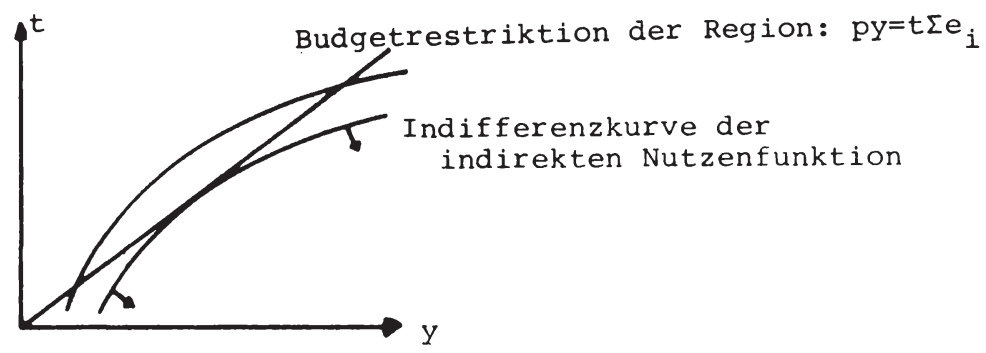

Die Präferenzrichtung der im Schaubild eingezeichneten Indifferenzkurve wird durch Pfeile angegeben. Die Alternativenmenge, aus welcher bei der Wahl Elemente ausgesucht werden könner, ist die im Schaubild eingezeichnete Gerade.

10) Die in einem Modell mit Einkommensteuern möglichen instabilen Situationen - "Arme" wandern "Reichen" nach - werden in $\mathrm{Ab}$ schnitt 5 dargestellt. 
Aus dem Vergleich der Definiton 2, 3a, 3b wird ersichtlich, daß bei seinem Emigrationskalkül ein Konsument die durch einen Regionenwechsel entstehenden externen Effekte nicht in Erwägung zieht. Auf diesen Mangel des "voting with one's feet" Konzepts machten insbesondere Buchanan und Wagner (1970), Buchanan und Goetz (1972) und Flatters et.al. (1974) aufmerksam. Aus diesem Grunde läßt sich die Ineffizienz (gemäß Paretokriterium) der bisher vorgestellten Tiebout-Konzepte (2), (3a), (3b) folgern. Diese Behauptung wird durch die folgenden Erläuterungen gezeigt. Wir spezifizieren die vorliegende ökonomie dadurch, daß identische Konsumenten und identische Produktionsfunktionen für Regionen mit gleicher Konsumentenzahl unterstellt werden. Das Beispiel sei so gewählt, daß die optimale Gemeindegröße $N / 2$ ist, die indirekte Nutzenfunktion $v_{i}\left(n_{k}\right)$ konkav ist und $v_{i}\left(n_{k}=0\right)<v_{i}\left(n_{k}=N\right)$ für $i=1, \ldots, N$ gilt. Mit dieser Information kann folgendes Schaubild gezeichnet werden. ${ }^{11)}$

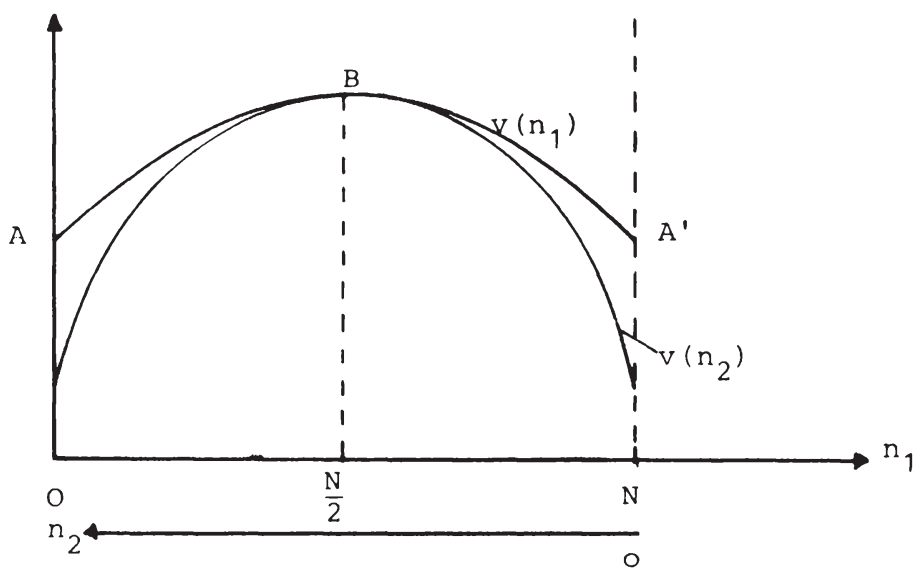

Schaubild 8

$v\left(n_{1}\right)$ bzw. $v\left(n_{2}\right)$ gibt den bei der Einwohnerzahl $n_{1} b z w \cdot n_{2}$ erzielten Nutzen. eines Konsumenten der Region 1 bzw. 2 wider.

11) Vgl. dazu etwa Flatters et.al.(1974). 
Dabei gibt ein Punkt auf der $n_{1}$-Achse mit $0 \leqq n_{1} \leqq N$ die Aufteilung der Bevölkerung auf Region 1 (mit $n_{1}$-Konsumenten) und Region 2 (mit $\mathrm{n}_{2}=\left(\mathrm{N}-\mathrm{n}_{1}\right)$-Konsumenten) an. Da die Regionen identisch sind, schneiden sich die $v\left(n_{1}\right)$ und $v\left(n_{2}\right)$-Kurven im punkt $\mathrm{N} / 2$.

Da die Konsumenten identisch sind, liegt ein dezentrales Gleichgewicht nach Konzept (2.a) sowie (2.b) genau dann vor, wenn gilt:

$$
\begin{aligned}
& v\left(n_{1}\right)=v\left(n_{2}\right) \text { oder } \\
& v\left(n_{i}=N\right) \geqq v\left(n_{j}=0\right) \text { mit } i \neq j ; i, j=1,2
\end{aligned}
$$

Damit sind die oben beschriebenen Punkte A,B,A' Gleichgewichte nach Konzept (2.a) sowie (2.b) und man erkennt unmittelbar die Suboptimalität der Gleichgewichtspunkte A und A'. Daher kann gefolgert werden, daß die in den Tiebout-Konzepten (2.a) und (2.b) den dezentralen Entscheidungseinheiten zur Verfügung gesteliten Informationen nicht hinreichend dafür sind, daß Konsumentenmobilität in einen Paretozustand führt. Das Informationsdefizit dieser Konzepte läßt sich anhand eines dem Schaubild 8 "aufgepropften" Tâtonnementprozesses interpretativ verdeutlichen. Betrachten wir eine Anfangssituation, in der Bezirk 1 über $\mathrm{N} / 2<\mathrm{n}_{1}<\mathrm{N}$ Konsumenten verfügt und die Bedingung (iv) der Definition $3 \mathrm{a} \mathrm{bzw}$. 3b erfüllt ist. In dieser situation erkennt ein Konsument der Region 2 durch Vergleich der steuersätze und der Angebote an lokalen öffentlichen Gütern in beiden Regionen, daß er in Region 1 den Nutzenindex $v\left(n_{1}\right)>v\left(n_{2}\right)$ realisieren kann. Folglich emigriert dieser Konsument in Gemeinde 1. Dieser Prozeß setzt sich so lange fort, bis der punkt A' in Schaubild 8 erreicht ist. Das Informationsdefizit bzw. die Informationsignoranz eines Konsumenten besteht dann darin, daß lediglich der Vergleich $v\left(n_{1}\right)$ mit $v\left(N-n_{1}\right)=v\left(n_{2}\right)$ und nicht das verhalten der anderen mit als Grundlage der Wanderungsentscheidung herangezogen wird. Als Konsequenz derartigen Verhaltens ergibt sich die Instabilität des Gleichgewichtspunktes $B$ und die bereits genannte suboptimalität der lokal stabilen Gleichgewichtspunkte A bzw. A'. 
3.3 Ein Dezentralisationskonzept mit Quasigemeinden

Zur Beseitigung der genannten Suboptimalitätseigenschaften des Tiebout-Mechanismus bieten sich, in Analogie zur Theorie der externen Effekte, interregionale Steuer- und Transfereingriffe einer Zentralregierung (sozialer Planer) an. Für den in Schaubild 8 diskutierten Fall ergäbe etwa eine Besteuerung $T_{i}$ eines Konsumenten der Region $i=1,2$ in Höhe von

$$
\mathrm{T}_{1}=v\left(\mathrm{n}_{1}\right)-\mathrm{v}\left(\mathrm{n}_{2}\right) \text { und } \mathrm{T}_{2}=-\frac{\mathrm{n}_{1}}{\mathrm{n}_{2}}\left[\mathrm{v}\left(\mathrm{n}_{1}\right)-\mathrm{v}\left(\mathrm{n}_{2}\right)\right]
$$

die Effizienz eines "Wanłerungsgleichgewichts". Denn $\mathrm{n}_{1}>\mathrm{n}_{2}$ impliziert $\mathrm{v}\left(\mathrm{n}_{1}\right)-\mathrm{T}_{1}<\mathrm{v}\left(\mathrm{n}_{2}\right)-\mathrm{T}_{2}$ und $\mathrm{n}_{2}>\mathrm{n}_{1}$ impliziert $\mathrm{v}\left(\mathrm{n}_{2}\right)-$ $\mathrm{T}_{2}<\mathrm{v}\left(\mathrm{n}_{1}\right)-\mathrm{T}_{1}$, womit ein Konsument dann bessergestellt wird, wenn er aus der "überbevölkerten" Region in die "unterbevölkerte" wandert.

Eine weitere Möglichkeit, die Effizienzeigenschaft der o.a. Gleichgewichts-Allokationen zu verbessern, besteht darin, die Informationsmenge der dezentralen Entscheidungseinheiten zu vergrößern. Dies kann dadurch geschehen, daß einem Konsumenten neben der bisher "beobachtbaren" Information quasi bisher "nicht-beobachtbare" Informationen zur Verfügung gestellt werden. Dezentrale Gleichgewichts-Konzepte, die eine erweiterte Informationsbasis fordern, liegen mit dem Konzept des "Equilibria with land developers" von Stiglitz (1977) und dem damit - in Bezug auf die Informationsbasis dezentraler Entscheidungseinheiten - eng verwandten Konzept des "competitive local public equilibrium" von Wooders (1978) vor. Bevor in der Definition 4 das Wooders Konzept beschrieben wird, soll folgende zusätzliche Forderung an unsere Modellökonomie gestellt werden.

Annahme c: Die Produktionsfunktion für das lokale öffentliche Gut wird für jeden Bezirk spezifiziert durch $\mathrm{y}^{\mathrm{k}}=\mathrm{h}\left(\mathrm{n}_{\mathrm{k}}\right) \mathrm{z}^{\mathrm{k}}$.

Damit wird gefordert, daß Regionen mit gleicher Bevölkerung über identische Produktionstechnologien verfügen.

Definition 4 (WOODERS) :

Eine Allokation $\left(J(K),\left(y^{k}, z^{k}\right),\left(x^{i}, y^{i}\right)\right)$ und ein Preissystem $p$ 
ist ein Gleichgewicht nach Konzept (3), wenn die Bedingungen (i), (ii), (iii) der Definition 2 erfült werden und zusätzlich gilt

(v) Für $i=1, \ldots, N$ gilt, daß $i \in J_{k}$ impliziert:

$p^{o} x^{i}+\frac{p^{k}}{n_{k}} y^{k}=e^{i}$ und $u^{i}\left(x^{i}, y^{k}, n_{k}\right) \geqq u^{i}\left(\widetilde{x}^{i}, y^{k \prime}, n_{k^{\prime}}\right)$
mit $p^{O} \tilde{x}^{i}+\frac{p^{o}}{n_{k^{\prime}}} z^{k^{\prime}}=e^{i}$ und $y^{k^{\prime}}=h\left(n_{k^{\prime}}\right) \cdot z^{k^{\prime}}$

für $J_{k}, \in J(K)$ und $1 \leqq 1 J_{k}, 1 \leqq N$.

Die Bedingung ( $v$ ) besagt, daß in einem gleichgewichtigen Zustand kein Konsument durch Bildung oder Gründung eines neuen Bezirkes seine Position verbessern kann. Die Bedingung (v) ist daher äquivalent zur Forderung, daß die unter (i), (ii), (iii) genannten Kriterien nicht nur für die vorliegende Bezirksstruktur $J(K)$ Gültigkeit besitzen, sondern auch "Quasi-Bezirke" mit einschließen. Somit steht einem Konsumenten bei einer Emigrationsentscheidung neben den bisherigen Informationen, nämlich den Steuersätzen und öffentlichen Gutsangeboten der existierenden Bezirke, zusätzliche Information über steuersätze, Preise und öffentliche Gutsangebote von "fiktiven" Bezirken zur Verfügung. Gefordert wird dann für eine gleichgewichtige situation, daß der jetzige standort des Konsumenten allen übrigen, also auch den "fiktiven", überlegen ist. Man erkennt, daß alle Gleichgewichte nach Konzept (3) der in Schaubild 8 dargestellten Spezifikation unserer Modellökonomie effizient sind. Dies ist so, da bei einer Bevölkerungsauftellung $\mathrm{n}_{1}=\mathrm{N}, \mathrm{n}_{2}=0$ jeder Konsument der Region 1 erkennt, daß ein "fiktiver" Bezirk, dem eine Bevölkerungszahl N/2 zugeordnet ist, ein besserer standort für ihn wäre. Folglich beschreibt der Punkt B in Schaubild 8 das einzige Gleichgewicht nach Konzept (3) dieses Beispiels.

Es läßt sich zeigen, daß bei Gültigkeit der Annahme c jedes Gleichgewicht nach Konzept (3) im Kern liegt und somit Pareto- 
optimal ist (Wooders (1978), Theorem 1). 12) Eine hinreichende Bedingung für die Existenz eines Gleichgewichts nach Konzept (3) ist gegeben, wenn Annahme c erfüllt ist und die Voraussetzungen des sogenannten "distinguished number Theorems" erfüllt werden (Wooders (1978), Theorem 2). 13) Anhand des folgenden Beispiels

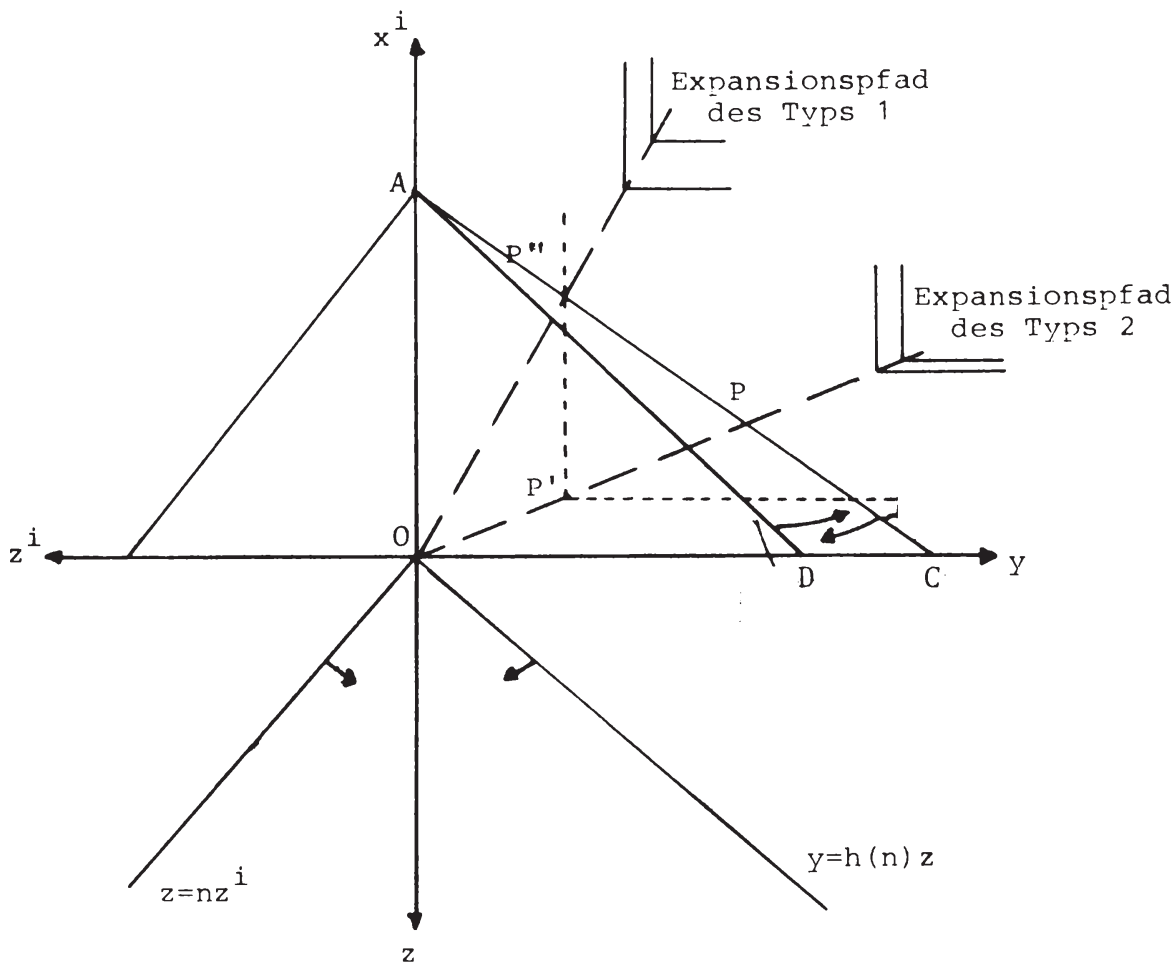

Schaubild 9

12) Der Kern ist die Menge erreichbarer Allokationen ( $(K)$, $\left.\left(y^{k}, z^{k}\right),\left(x^{i}, y^{i}\right)\right)$, bei denen für keine Gruppe $S$ c N von Konsumenten eine erreichbare Allokation $\left(\left(y^{S}, z^{S}\right),\left(x^{i}, y^{i}\right)\right)$ exisitert, so daß $u^{i}\left(x_{s}^{i}, y^{s}, n_{s}\right)>u^{i}\left(x^{i}, y^{k}, n_{k}\right)$ für alle $i \in s$ mit $|s|=n_{s}$ und $y^{s}=h\left(n_{s}\right)\left(\Sigma w^{i}-\Sigma x^{i}\right)$ gilt.

Der Kern ist somit die Menge der erreichbaren Allokationen, die keine Gruppe im Alleingang verbessern kann.

13) Seien $\mathrm{m}^{\prime}, \mathrm{m}^{\prime}$ und $\mathrm{N}$ ganze Zahlen. Das "distinguished number Theorem" gibt dann Bedingungen an, unter denen die ganze $\mathrm{Zahl}$ $\mathrm{N}$ in ganzzahlige Vielfache von $\mathrm{m}^{\prime}$ und $\mathrm{m}$ " zerlegbar ist. 
sollen informal Bedingungen erläutert werden, unter denen das "distinguished number Theorem" gilt und die gleichgewichtige Bedürnisstruktur aus homogenen Bezirken besteht. Unter homogenen Bezirken werden Bezirke verstanden, in denen jeweils nur Konsumenten des gleichen Typs wohnen.

Wir gehen davon aus, daß in der ökonomie zwei Typen von Konsumenten existieren. Beide Typen verfügen über gleiches Einkommen und unterscheiden sich damit lediglich durch ihre präferenzen. Bezüglich der präferenzen wird unterstellt, daß die beiden Güter streng komplementär sind und keine crowding-Effekte im Konsumbereich existieren. Im produktionsbereich liegt ein crowding-Effekt vor, wobei gilt, daß bei den Gruppengrößen $n^{*}$ und $n^{*}+1$ die Budgetmengen $B_{i}(n)$ eines Konsumenten identisch sind und die Fläche unter der Budgetgeraden ihr Maximum erreicht. Dann ist $B_{i}\left(n^{*}\right)$ und ${ }_{i}\left(n^{*}+1\right)$ im vorstehenden Schaubild durch das Dreieck OAC darstellbar und Dreieck OAD beschreibt $B_{i}(\bar{n})$ für $\bar{n} \neq n^{*}$ und $\bar{n} \neq n *+1$.

Da für beliebiges $n=1, \ldots, N$ gilt $B_{i}(n) \subset B_{i}\left(n^{*}\right) \equiv\left\{\left(x^{i}, y\right) \epsilon\right.$ $\epsilon R_{+}^{2}: x^{i}+\frac{1}{n^{*}} z=w^{i}$ und $\left.y=h\left(n^{*}\right) \cdot z\right\}$ und $B_{i}\left(n^{*}+1\right)=B_{i}\left(n^{*}\right)$, sind $n^{*}$ und $\left(n^{*}+1\right)$ die optimalen Bezirksgrößen für Typ 1 und Typ 2. Wird mit $\mathrm{N}_{i}$ die Anzahl an Konsumenten des Typs $i=1,2$ in unserer ökonomie bezeichnet und ist $N_{i}>\left(n^{*}-1\right) n^{*}$ für $i=1,2$ erfüllt, ist das "distinguished number Theorem" erfüllt. Unter dieser Voraussetzung existiert ein Gleichgewicht nach Konzept (3) undim Gleichgewicht liegen homogene Bezirke vor. Aus obigem Schaubild ist erkennbar, daß der Punkt $P$ " die gleichgewichtige Allokation im Bezirk des Typs 1 beschreibt. Würde ein Konsument des Typs 2 in den Bezirk des Typs 1 emigrieren, ergäbe sich für ihn das dem Punkt $\mathrm{P}^{\prime}$ zugeordnete Nutzenniveau. Analog impliziert eine Emigration eines Konsumenten des Typs 1 in einen Bezirk des Typs 2 eine Verschlechterung seiner Position. Anhand des Schaubildes 9 ist weiterhin zu erkennen, daß für dieses Beispiel kein Konsument durch Freifahrerverhalten ein Vorteil erringen kann. Dies ist so, da alle individuellerreichbaren Konsumkombinationen in einem Gleichgewicht für einen Konsumenten in der Menge $B_{i}(n)$ liegen und der Punkt $\mathrm{P}$ bzw. P" die jeweils beste Konsumkombina- 
tion für einen Konsumenten des Typs 1 bzw. Typs 2 ist. Unterstellt $\operatorname{man} N_{i}>\left(n^{*}-1\right) n^{*}$, so kann gezeigt werden, daß ganze Zahlen $m_{i}^{\prime}, m_{i}^{\prime \prime}$ derart existieren, daß $m_{i}^{\prime} n^{*}+m_{i}^{\prime \prime}\left(n^{*}+1\right)=N_{i}$ für $i=1,2$ gilt. Daher kann $N_{i}$, die Anzahl der Konsumenten jedes 'i'yps in $m_{i}^{\prime}$ und $m_{i}^{\prime}$ Gruppen der optimalen Größe $n^{*}$ und $(n *+1)$ zerlegt werden. Die gleichgewichtige Bezirksstruktur $J(K)$ ist daher so strukturiert, $d a \beta$ es $m_{i}^{\prime}$ Bezirke mit der Bevölkerung $n^{*}$ unà mi. Bezirke mit der Bevölkerung $(n *+1)$ gibt.

Die Nichtexistenz eines Gleichgewichtes gemäß Konzept (3) bei Nichterfüllung der Bedingung $N_{i}>\left(n^{*}-1\right) n^{*} l a ̈ ß t ~ s i c h$ an folgendem Beispiel zeigen. Wir gehen von einer ökonomie aus, in der genau 3 Konsumenten eines Typs leben und die optimale Gruppengrösse mit $n^{*}=2$ gegeben ist. Für eine derartige ökonomie existiert kein Gleichgewicht gemäß Konzept (3), denn bei näherer Analyse cier möglichen Bezirksstrukturen wird deutlich, daß keine dieser Strukturen in einem Gleichgewichtszustand vorliegen kann. Schliessen sich alle drei Konsumenten zu einem Bezirk zusammen, wird die Bedingung $(v)$ der Definition 4 nicht erfüllt, denn ein Konsument der Dreiergruppe wäre in einem Quasi-Bezirk mit 2 Konsumenten bessergestellt. Analog gilt für die Fälle, daß zwei Konsumenten einen Bezirk bilden und jeweils ein konsument ein Bezirk bildet. weht man davon aus, daß in großen ökonomien mit lokalen öffentlichen Gütern die optimale Gruppengröße in bezug auf die Gesamtzahl der Konsumenten eines Typs klein ist, kann erwartet werden, daß für große Ökonomien Gleichgewichte nach Konzept (3) existieren.

Ein weiteres Beispiel der Nichtexistenz eines Gleichgewichtes rach Konzept (3) - und damit eine Einschätzung der Qualität des Tiebout'schen "voting with one's feet" Konzepts bei hinreichender Informationsbasis dezentraler Entscheidungseinheiten liefert die Verallgemeinerung der Annahme c. Geht man davon aus, daß sich Bezirke mit gleicher Bezirksgröße durch unterschiedliche Proauktionstechniken unterscheiden, läßt sich folgender Fall konstruieren. Wir gehen von einer ökonomie mit einem Konsumententyp 
aus. Weiterhin sei eine Region 1 mit einer Produktionstechnik $y^{1}=h^{1}\left(n_{1}\right) z^{1}$ gegeben. Alle übrigen Regionen verfügen über die Produktionstechnik $\mathrm{y}^{\mathrm{k}}=\mathrm{h}\left(\mathrm{n}_{\mathrm{k}}\right) \mathrm{z}^{\mathrm{k}}$ mit $\mathrm{h}^{1} \neq \mathrm{h}$. Das Beispiel sei so gewählt, daß die indirekte Nutzenfunktion $v\left(n_{1}\right)$ bzw. $v\left(n_{k}\right)$ unseres "Einheitskonsumenten" den im nachstehenden Schaubild eingezeichneten Verlauf aufweist.

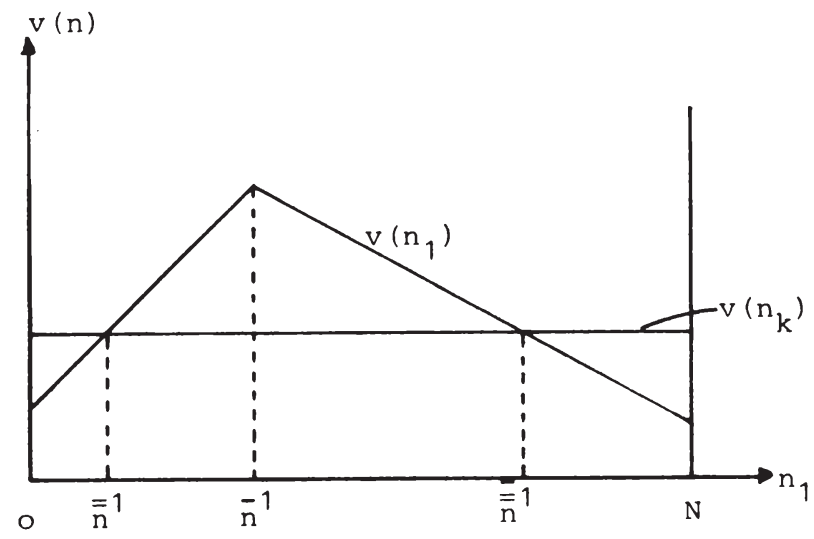

Schaubild 10

Da der Gesamtbevölkerung lediglich ein Bezirk mit der Technologie $h^{1}$ zur Verfügung steht, können höchstens $\bar{n}_{1}$ Konsumenten den Nutzenindex $v\left(\bar{n}_{1}\right)$ realisieren und $\left(N-\bar{n}_{1}\right)=\bar{n}_{k}$ den Nutzenindex $v\left(\bar{n}_{k}\right)$. Da $v\left(\bar{n}_{1}\right)>v\left(\bar{n}_{k}\right)$ gilt, kann sich ein konsument nicht dadurch verbessern, daß er einen Quasi-Bezirk mit Technologie ${ }^{1}$ gründet, sondern nur dadurch, daß er in Region 1 emigriert. Daher ist die Bezirksstruktur $\left|J_{1}\right|=\bar{n}_{1}$ und $\left|J_{k}\right|=N-\bar{n}_{1}$ keine gleichgewichtige Bezirksstruktur. Analoges gilt für jede andere Aufteilung der Konsumenten.

\subsection{Tiebout-Gleichgewicht mit Kopfsteuern}

Im folgenden wird untersucht, ob in einem Gleichgewicht gemä $\beta$ Definition $3 \mathrm{a} \mathrm{bzw}$. 3 homogene oder heterogene Gemeindestrukturen vorliegen. Um die Analyse einfach zu halten, wird davon ausgegangen, daß alle Konsumenten die gleiche Nutzenfunktion haben, während sie sich in ihren Einkommen unterscheiden können. Die 
Wanderungsentscheidung eines Konsumenten hängt dann davon $a b$, ob er ein höheres Nutzenniveau erreichen kann, wenn er in eine Gemeinde zieht, deren Bewohner über ein höheres (bzw. niedrigeres) Einkommen verfügen. ${ }^{14)}$

Betrachten wir den Fall, daß alle Ķonsumenten einer Gemeinde $k=1, \ldots, k$ das gleiche Einkommen haben, d.h. eine homogene Gemeindestruktur vorliegt. In der Ausgangslage befindet sich ein Konsumente $i$ aus Gemeinde $k$ in seinem Nutzenmaximum bei gegebenem Einkommen $e^{i}$, (Punkt $P_{i}$ in Schaubild 11). Wechselt er in eine Gemeinde $k^{\prime}$, in der die Einwohner das Einkommen $e^{j}>e^{i}$ haben, $p^{k}=$ $\mathrm{p}^{\mathrm{k}^{\prime}}$ gilt und $\mathrm{y}$ ein normales Gut ${ }^{15)}$ ist, dann folgt bei einer Kopfsteuer und identischen Präferenzstrukturen $y^{j}>y^{i}$. Dabei bezeichnen $\mathrm{y}^{i}$ und $\mathrm{y}^{j}$ die jeweils beste Allokation des öffentlichen Gutes in der homogenen Gemeinde k bzw. k'.

Durch Wanderung in eine Gemeinde $\mathrm{k}^{\prime}$, in der die Einwohner ein höheres Einkommen als in $\mathrm{k}$ haben, befindet sich Konsument $i$ nicht in seinem Nutzenmaximum $\mathrm{P}_{i}$, sondern im Punkt $\hat{\mathrm{P}}_{i}$. Dies ist so, da

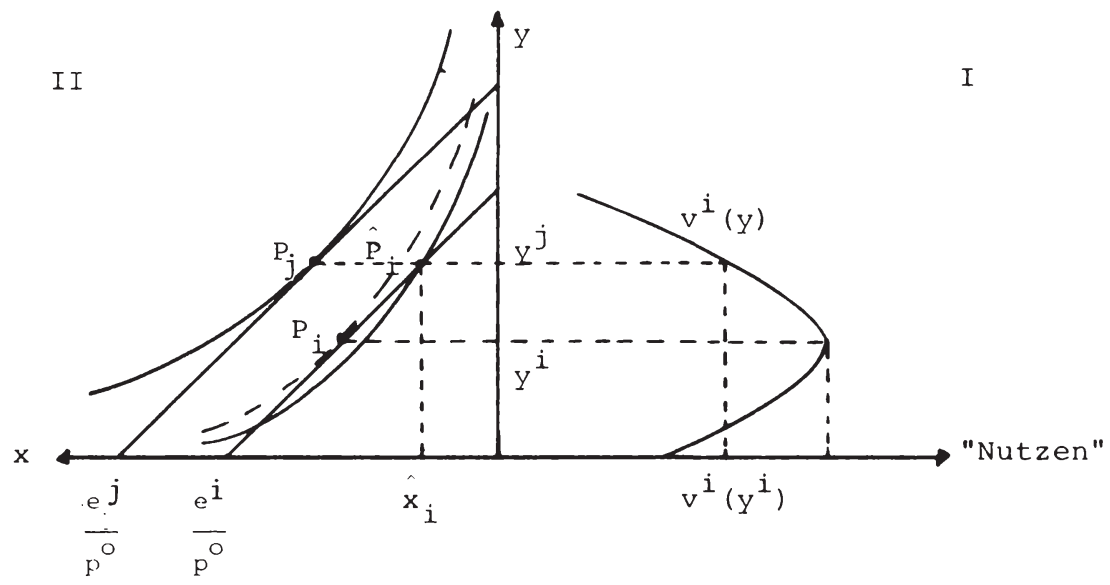

Schaubild 11

14) Im folgenden wird nur für ein höheres Einkommen argumentiert. Für ein niedrigeres ergibt sich die analoge Argumentation.

15) Ein normales Gut liegt dann vor, wenn steigendes Einkommen steigenden Konsum des Gutes impliziert. 
er für ein höheres y einen größeren Teil seines Einkommens zur Finanzierung des öffentlichen Gutes aufwenden muß, als für ihn nutzenmaximal ist. Der Nachteil des Emigrierens aus einer homogenen Gemeinde wird in Schaubild 11 durch den im I. Quadranten eingezeichneten Verlauf der indirekten Nutzenfunktion $v^{i}(y)$ deutlich.

Daraus ersieht man, daß es für i nur einen wert von y gibt, der seinen Nutzen maximiert bei gegebenem preissystem $p$ und Einkommen $e^{i}$. Das bedeutet aber, daß kein konsument seine Gemeinde verläßt, so daß das durch Definition 3a bzw. 4 beschriebene Gleichgewicht eine homogene Gemeindestruktur erfordert.

Für die Stabilität eines solchen Gleichgewichtes gilt: Wir gehen davon aus, $d a \beta$ in der Ausgangslage heterogene Gemeinden vorliegen. Anhand des Schaubildes 11 kann gezeigt werden, daß sich homogene Gemeinden bilden. Sei $y^{j}$ die von der Gemeinde $k$ angebotene Menge des öffentlichen Gutes. 16) Die reichere Bevölkerungsgruppe befindet sich dann im optimum $\mathrm{P}_{j}$, während sich die ärmere außerhalb ihres optimums $P_{i}$ befindet, nämlich in $\hat{P}_{i}$. Für ein Individuum $i$ lohnt es sich dann, in eine Gemeinde zu wandern, in der ein niedrigeres $y$ als $y^{i}$ angeboten wird, d.h. in eine Gemeinde zu wandern, in der die ärmere Bevölkerung in der Mehrheit ist. Deshalb wird Individuum i so lange wandern, bis es eine Gemeinde gefunden hat, in der seine Einkommensklasse die Mehrheit stellt, da es dann in seinem Nutzenmaximum liegt. Die gleiche Argumentation gilt für alle konsumenten, so daß sich die Konsumenten zu homogenen Gemeinden zusammenschließen. Für die Finanzierung durch Kopfsteuer ist also das Gleichgewicht nach Konzept (2a) bzw. (3) stabil und hat darüber hinaus noch die Eigenschaft, daß sich die Konsumenten in homogene Gruppen aufteilen.

16) Für die Zeichnung kann angenommen werden, daß in $\mathrm{k}$ zwei Arten von Konsumenten wohnen, die reichere Gruppe größer ist als die ärmere und $y^{j}$ über Mehrheitswahl bestimmt wurde. 


\subsection{Tiebout-Gleichgewicht mit Einkommensteuern}

Nachdem die Implikationen von Kopfsteuern erörtert wurden, wird nachstehend die Problematik von Einkommensteuern zur Finanzierung lokaler öffentlicher Güter aufgegriffen. Dabei wird das Problem der optimalen Gemeindegröße als gelöst vorgegeben.

\subsubsection{Kurzsichtige Nutzenmaximierung}

Wir gehen davon aus, daß alle Konsumenten ihr gesamtes Einkommen für den Konsum des privaten Gutes $x$ und die Steuerzahlung zur Finanzierung des öffentlichen Gutes $y$ ausgeben. Um die Analyse einfach zu halten, wird wiederum unterstellt, daß alle Konsumenten die gleiche Nutzenfunktion haben, während sie sich in ihren Einkommen unterscheiden können. Die Anzahl der gegebenen Gemeinden ist sehr groß. Sei $k$ eine Gemeinde, in der $n_{k}$ Konsumenten leben. Wir betrachten jetzt das Problem, wie sich der Nutzen eines Individuums 1 ändert. Diese Vorgehensweise ist äquivalent zu der Frage, wie ein Individuum ohne Berücksichtigung der Auswirkungen auf andere Individuen daraus Nutzen ziehen kann, wenn es in eine andere Gemeinde wandert, die sich nur marginal von seiner jetzigen unterscheidet. (Vgl. Wheaton (1975)). Die Nutzenänderung des $i$ bei einer Einkommensänderung des 1 ist:

$$
\frac{d u^{i}}{d e^{1}}=u_{x}^{i} \frac{d x^{i}}{d e}+u_{y}^{i} \frac{d y}{d e} 1
$$

und daher vom Vorzeichen der Ausdrücke $\frac{\mathrm{dx}}{\mathrm{de}}{ }^{1}$ und $\frac{\mathrm{dy}}{\mathrm{de}} 1$ abhängig.

[)a für die Budgetgerade des i gilt:

$e^{i}=\frac{e^{i} p}{\sum e^{i}} y+p^{o} x^{i}$

und totale Differentiation nach $\mathrm{e}^{1}$

$0=e^{i} p\left(\frac{\frac{d y}{d e} 1 \sum e^{i}-y}{\left(\Sigma e^{i}\right)^{2}}+p^{o} \frac{d x^{i}}{d e^{T}}\right)$

ergibt, folgt

$\frac{d x^{i}}{d e^{1}}=\frac{e^{i} p y}{p^{0}\left(\sum e^{i}\right)^{2}}-\frac{e^{i} p}{p^{0} \sum e^{i}} \frac{d y}{d e^{1}}$ 
Durch Einsetzen in (12) erhält man

$$
\frac{d u^{i}}{d e^{1}}=u_{x}^{i} \frac{e^{i} p y}{p^{0}\left(\Sigma e^{i}\right)^{2}}+\left(u_{y}^{i}-\frac{e^{i} p}{p^{0} \Sigma e^{i}}\right) \frac{d y}{d e^{1}}
$$

Berücksichtigt man, daß der Einkommensteuersatz $t=\frac{p y}{\sum e^{i}} i s t$ und setzt man $\tilde{\mathrm{p}}^{\mathrm{i}}=\frac{\mathrm{e}^{\mathrm{i}} \mathrm{p}}{\varepsilon \mathrm{e}^{i}}$, kann man schreiben

(13') $\frac{d u^{i}}{d e^{1}}=u_{x}^{i}\left[\frac{t}{p^{0}} \frac{e^{i}}{\Sigma e^{i}}+\left(\frac{u_{y}^{i}}{u_{x}^{i}}-\frac{\tilde{p}^{i}}{p^{0}}\right) \frac{d y}{d e^{1}}\right]$

Im folgenden wird von normalen Gütern ausgegangen, d.h. für $e^{j}>e^{i}$ gilt $\left(x^{i}, y^{i}\right)<\left(x^{j}, y^{j}\right)$, wobei $\left(x^{i}, y^{i}\right)$ und $\left(x^{j *}, y^{j}\right)$ nutzenmaximale Güterbündel beim Einkommen $e^{i} b z w . e^{j}$ sind. Die Richtung der Nutzenänderung des $i$ hängt einerseits vom Vorzeichen von $\left(\mathrm{dy} / \mathrm{de}^{1}\right)$ und andererseits davon $\mathrm{ab}, \mathrm{ob}$ $\left(u_{y}^{i} / u_{x}^{i}\right) \geqslant\left(\tilde{p}^{i} / p^{0}\right)$ ist. Betrachten wir zuerst $\left(d y / d e^{1}\right):$ Durch die steigerung des Einkommens von Individuum 1 wird für Individuum $i$ das öffentliche Gut y billiger, da die Summe der Einkommen steigt. D.h. der Preis $\mathrm{p}^{i}$ sinkt und damit dreht sich die Budgetgerade im punkt $\left(e^{i} / p^{0}\right)$ nach außen. (Vgl. Schaubild 12). Da von normalen Gütern ausgegangen wird, steigt die nutzen-

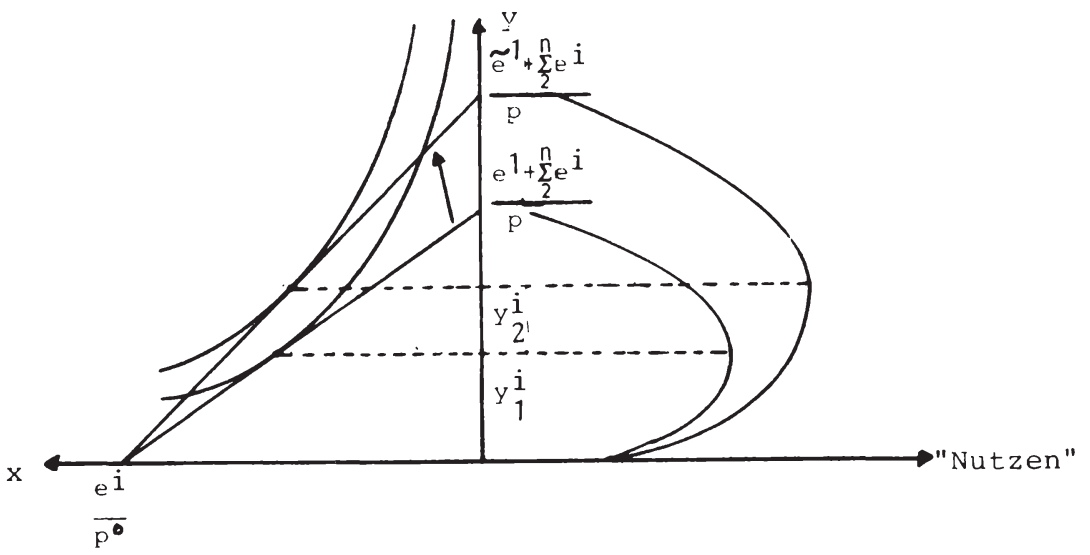

Schaubild 12 
maximale Menge des öffentlichen Gutes für Individuum i. Da das in Region $\mathrm{k}$ produzierte $\mathrm{y}^{\mathrm{k}}$ über Mehrheitswahl bestimmt wird, und obige überlegungen für alle i gilt, steigt die produktion des öffentlichen Gutes in Region $k$, so daß dy/de ${ }^{1}>0$ gilt.

Jetzt werden die Implikationen von $\left(u_{y}^{i} / u_{x}^{i}\right) \geqq\left(\tilde{p}^{i} / p^{0}\right)$ untersucht. Im Optimalpunkt des Konsumenten $i,\left(x^{i}, y^{i}\right)$, gilt: $\left(u_{y^{*}}^{i} / u_{x^{*}}^{i}\right)=\left(\tilde{p}^{i} / p^{0}\right) d \cdot h$.

$$
\frac{u_{y}^{i}}{u_{x}^{i}} \geqq \frac{u_{y^{*}}^{i}}{u_{x^{*}}^{i}}
$$

Daraus folgt, daß die steigung der Indifferenzkurzve in $\left(x^{i}, y\right)$ steiler ist als die Budgetgerade. Für welche Einkomrnensklassen gilt dies? Da wir annahmegemäB davon ausgehen, daß Y ein normales Gut ist und alle die gleiche Präferenzstruktur haben, gilt, daß reichere Einwohner ein höheres optimales y haben als ärmere. Aber dann muß für die ärmere Bevölkerung $\left(u_{y}^{i} / u_{x}^{i}\right) \geqq\left(u_{y^{*}}^{i} / u_{x^{*}}^{i}\right)$ gelten. Greifen wir uns z.B. zwei Einkommensklassen $e_{I}$ und $e_{I I}$ mit $e_{I}>e_{I I}$ aus einer Gemeinde mit mehreren Einkommensklassen heraus, wobei weder $Y_{I}^{*}$ noch $Y_{I I}^{*}$ das Mehrheitswahlgleichgewicht sein soll, sondern dieses zwischen $y_{I}^{*}$ und Y* liegt. Dies ist in Schaubild 13 dargestellt. Dann schneidet die Indifferenzkurve die Budgetgerade eines Individuums der Einkommensklasse I (II) unterhalb (oberhalb) des Optimalpunktes P* (P* ${ }_{I}^{*}$ ). (Vgl. Schaubild 13). Da die Indifferenzkurven konvex sind, gilt für die reichere (ärmere) Gruppe I (II), daß $\frac{u_{y}^{I}}{u_{x}^{I}} \leqq \frac{u_{y^{*}}^{I}}{u_{x^{*}}^{I}} \quad\left(\frac{u_{y}^{I I}}{u_{x}^{I I}} \geqq \frac{u_{y^{*}}^{I I}}{u_{x^{*}}^{I I}}\right)$.

Bei einer Einkommensteuer gilt also, daß die ärmere Bevölkerung in Gemeinden wandert, in denen reichere die Mehrheit stellen, da sie dadurch ein höheres Nutzenniveau erreichen können. Deshalb kann ein Gleichgewicht nach Konzept (2b), auch wenn das Problem der optimalen Gemeindegröße in der Ausgangslage gelöst wäre, nicht erreicht werden. Die ärmere Bevölkerung drängt in die 
Gemeindenmit reicherer Bevölkerung, wenn alle Einwohner ein extrem kurzsichtiges Nutzenmaximierungskalkül haben.

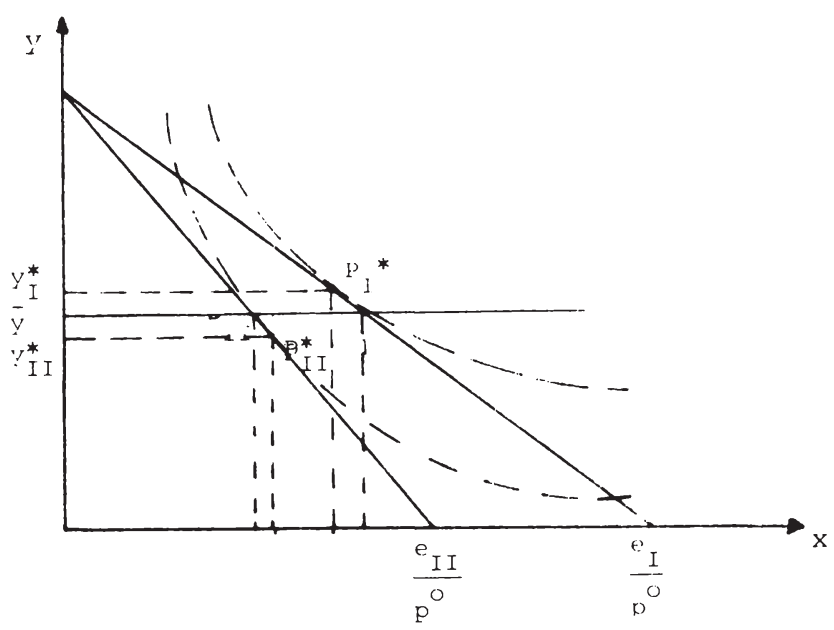

Schaubild 13

\subsubsection{Erweiterte Informationsbasis}

Die Ausführungen des vorigen Abschnittes zeigen, daß durch die in Konzept ( $2 b$ ) spezifizierten Entscheidungsregeln keine gleichgewichtige Bezirksstruktur zustande kommen kann. Im folgenden wird nun diskutiert, ob durch eine Erweiterung der Informationsbasis für zumindest einen Teil der Bevölkerung gleichgewichtige, homogene Bezirksstrukturen auftreten können. Dazu betrachten wir eine ökonomie, in der es zwei Arten von Konsumenten gibt, die sich nur im Einkommen unterscheiden. Die Gesamtzahl der Bevölkerung läßt sich so in zwei Regionen aufteilen, daß beide Regionen ihre optimale Einwohnerzahl $\mathrm{n}_{\mathrm{A}}$ bzw. $\mathrm{n}_{\mathrm{R}}$ haben, wobei die Einwohner der Region $A$ zu der Klasse mit niedrigem Einkommen gehören, während in Region $R$ die Konsumenten mit hohem Einkommen wohnen.

\section{Fall A: Kurzsichtiges Verhalten der Armen}

Unterstellen wir den einzelnen armen Konsumenten kurzsichtiges Verhalten, d.h. sie betrachten nur ihren eigenen Nutzenzuwachs 
durch die Veränderung in Region $R$, während sie die Nutzeneinbußen der reichen Konsumenten nicht in ihr Kalkül mit einbeziehen. Die reichen Konsumenten dagegen sollen mehr Information haben, und zwar sollen sie das Entscheidungskalkül des Armen genau kennen. In Schaubild $14 \mathrm{a}$ ist das Entscheidungskalkül eines Individuums aus Region A dargestellt. Aus der Analyse des vorigen Abschnittes folgt, daß sich der Arme durch Wanderung in die reiche Gemeinde besserstellt. Dies ist durch Drehung der Budgetgeraden des Armen um den punkt $\bar{x}_{A}$ in Schaubild 14a dargestellt. In Schaubild $14 \mathrm{~b}$ sind die Auswirkungen der Emigration eines Bewohners der Region $\mathrm{A}$ in die Region $\mathrm{R}$ skizziert. Die Budgetgerade $\bar{x}_{R} \bar{y}_{R}$ aller Reichen dreht sich nach unten, da durch einen zusätzlichen Einwohner die optimale Gemeindegröße überschritten wird. D.h. die Bewohner der Region $\mathrm{R}$ würden durch einen zusätzlichen Mitbewohner Nutzeneinbußen erleiden.
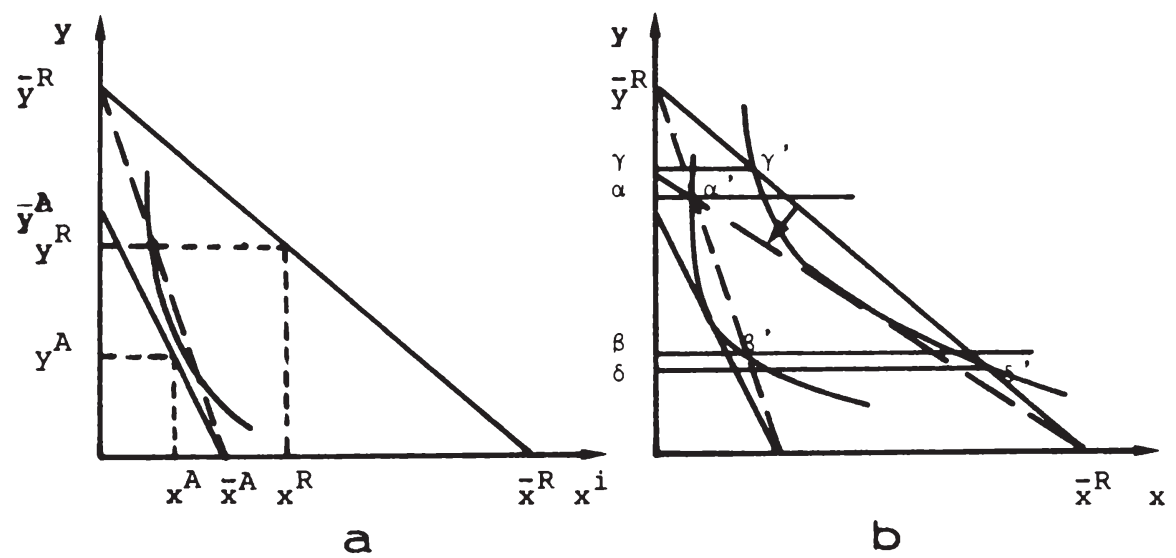

Schaubild 14

17) Vgl. die Analyse in Abschnitt 2.2, S. $102 \mathrm{ff}$. 
Da die Einwohner der Region $\mathrm{R}$ die Charakteristika der Armen kennen, besitzen sie die Möglichkeit, durch entsprechende Festlegung eines öffentlichen Güterangebotes eine Immigration eines Individuums von Region $A$ nach $R z u$ verhindern. Dies ist in Schaubild 14b durch die dort eingezeichneten Ausschlußpunkte kenntlich gemacht.

Betrachten wir den Punkt $\alpha$ : Für Individuum i lohnt es sich, gemä $\beta$ seinem Kalkül, nach Region $\mathrm{R}$ zu ziehen, wenn das in $\mathrm{R}$ bereitgestellte $y$ zwischen $\alpha$ und $\beta$ liegt. Bietet Region $R$ ein geringfügig nöheres $y$ als $\alpha$ an, bedeutet dies für $i$ eine Nutzeneinbuße im Vergleich zu seinem Optimalpunkt in Region A, d.h. es wird in seiner Region bleiben. Andererseits hat ein Individuum aus $\mathrm{R}$ eine geringere Nutzeneinbuße, wenn y zwischen $\alpha$ und $\gamma$ liegt, als wenn ein zusätzlicher armer Konsument in Region $R$ einwandert. D.h. daß in diesem Fall eine gleichgewichtige, homogene Bezirksstruktur vorliegt und dieses Gleichgewicht Pareto-optimal ist. Für den Punkt $\beta$ gilt eine analoge Argumentation. Gilt $y<\beta$, haben die Reichen das öffentliche Gut teilweise privatisiert, d.h. sie substituieren es durch $\mathrm{x}$. Dies ist bei vielen lokalen öffentlichen Gütern vorstellbar. Nehmen wir z.B. die Schulausbildung von Kindern. Es wird nur noch ein Grundkurs öffentlich angeboten, während die weitere Ausbildung durch Privatlehrer erteilt wird. Geht dann ein Armer in die Region $R$, ist er gezwungen, einen Teil seiner Ausgaben für private Güter ( $x$ wird hier als Güterbündel interpretiert) für die Ausbildung auszugeben, um gemäß seiner Nutzenfunktion zu handeln. Er ist jedoch nicht in der Lage, sein iNutzenniveau aufrecht $z u$ erhalten, wenn $y<\beta i s t$.

Fall B: Kurzsichtiges Verhalten der Reichen

Gehen wir jetzt davon aus, daß die Armen über die größere Information verfügen, während die Reichen nur kurzsichtig ihre eigene Nutzenmaximierung anstreben. Dann kann folgendes Diagramm gezeichnet werden. (Schaubild 15).

Ist $y$ ein normales Gut, kann der Fall eintreten, daß der neue Optimalpunkt $\mathrm{P}$ der Region $\mathrm{R}$ oberhalb von $\alpha$ liegt und damit der Arme, aufgrund seiner Information, nicht in die Region $R$ wandert, 


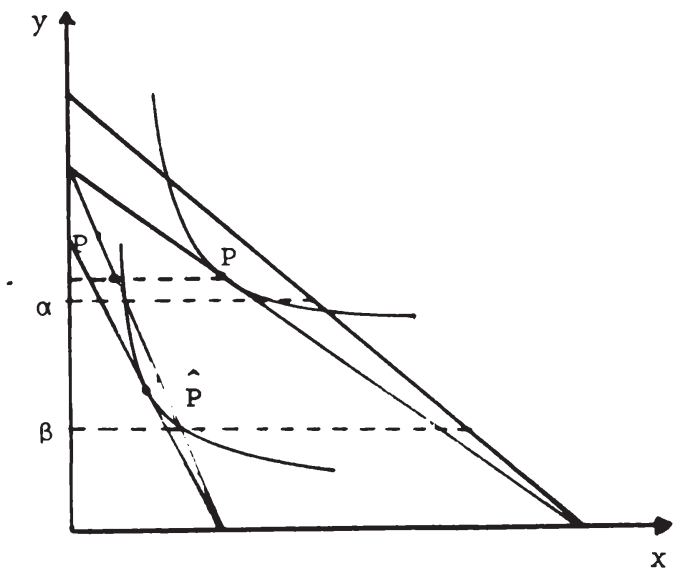

Schaubild 15

da er sich in Region A besserstellt. Aus Schaubild 15 ist ersichtlich, daß der Punkt $\mathrm{P}^{\prime}$, der dem Güterbündel des Armen zugeordnet ist, wenn er in der Region $\mathrm{R}$ leben würde, auf einer niedrigeren Indifferenzkurve liegen würde als der Optimalpunkt $\hat{\mathrm{P}}$ der Region A. Analog zum "kurzsichtigen Verhalten der Armen" liegt auch hier ein indirekter Ausschlußmechanismus vor. Eine analoge Argumentation kann gelten, wenn $y$ ein absolut inferiores Gut ist, d.h. der neue Optimalpunkt eines Individuums aus $R$ liegt unterhalb von $\beta$.

\subsubsection{Andere Möglichkeiten der Abgrenzung}

Durch die eben beschriebenen Arten der Abgrenzung können sich einzelne Gruppen $z u$ ihrem Vorteil in homogenen Gemeindenzusammenschließen. Diese Abgrenzung ist von indirekter Art, d.h. sie wird nur über die lokalen öffentlichen Güter und der damit verbundenen Steuerzahlung betrieben. In der Literatur wird auch eine direkte Art von Abgrenzung diskutiert, das sogenannte "zoning". 18) Dabei 18) Vgl. z.B. Stiglitz (1977, 320-324), Fischel (1980). 
wird von der armen Bevölkerung verlangt, wenn sie in eine reiche Region ziehen will, daß sie eine bestimmte Menge eines privaten Gutes kaufen muß, um dort ansässig $\mathrm{zu}$ werden. Das Standardbeispiel ist hier der Wohnraum, der gekauft bzw. gemietet werden muß, wenn man in ein bestimmtes Wohngebiet zieht. Auch hier ist die Abgrenzung dann erfolgreich, wenn von dem Armen soviel seines Einkommens für Wohnraum abverlangt wird, daß sein verbleibendes Einkommen nicht ausreicht, die entsprechenden öffentlichen und privaten Güterausgaben zu finanzieren, um sein Nutzenniveau zu halten.

\section{Schlußbetrachtung}

Der im ersten Abschnitt dieses Beitrags dargestellte Vorschlag Tiebout's zur Allokation lokaler öffentlicher Güter über Konsumentenwanderungen stellt einen Lösungsansatz des Allokationsproblems dar, bei dem die Rolle staatlicher Eingriffe reduziert werden auf die Schaffung geeigneter Rahmenbedingungen, etwa durch eine Politik, die zur Mobilitätsförderung beiträgt. Auf die Frage, inwieweit sich ein derart dezentrales Allokationsverfahren eignet, eine allokationstheoretisch befriedigende Antwort zum Problem 1okaler öffentlicher Güter zu geben - also die Frage der Allgemeinheit der Anwendungsbedingungen - wurde versucht, in einem einfachen Zwei-Gütermodell eine Teilantwort zu geben. Da Tiebout selbst keine eindeutige Definition eines gleichgewichtigen Zustands liefert, wurden mit den Konzepten 1,2,3 mögliche dezentrale Gleichgewichte diskutiert. Dabei wurde anhand der von Konzept 1 bis 3 ansteigenden Informationsvoraussetzungen der dezentralen Entscheidungseinheiten deutlich, daß die Analogie der Wahl eines SteuerGüterpakets mit dem "Markt-Test" eines Gutes nicht aufrechterhalten werden kann. Externe Effekte individueller Wanderungsentscheidungen machen es erforderlich, daß der konsument über nicht-existierende "Quasi-Gemeinden" informiert wird, damit dezentrale Gleichgewichte paretooptimal sind (Konzept 3 in Abschnitt 3.3). Wird darüber hinaus gefordert, daß die öffentlichen Güterbündel verschiedener Gemeinden nicht über Kopf-, sondern Einkommensteuern finanziert werden, treten zusätzlich erhebliche Einschränkungen 
der Allgemeinheit des Tiebout-Resultats auf. Da, wie bereits betont, in einem einfachen Modellzusammenhang argumentiert wurde, blieben eine Vielzahl von Faktoren, wie etwa Finanzausgleich, Mobilitätsbereitschaft, Mobilitätsunterschiede bei Arbeit, Kapital und technischem Wissen sowie "institutionelle Aspekte" unberücksichtigt. Mit den Worten von Sandler, Tschirhart (1980, 1507) läßt sich resumieren: "With all of these requirements pareto optimality is an unlikely real-world solution to the Tiebout model, although it is a theoretical possibility". 
Anhang:

Das repräsentative Individuum habe folgende Nutzenfunktion:

(A. 1) $u(x, y, n)=\left[\frac{1}{257 x^{4}}+\frac{256}{257 y}\right]^{-\frac{1}{4}}$

d.h. $u_{3}=0$, crowding im Konsumbereich ist ausgeschlossen.

19) Die Nutzenfunktion hat ein relativ kompliziertes Aussehen, da die $v(n)$-Funktion nur dann ein Maximum hat, wenn das öffentliche und das private Gut wenig substituierbar sind. In unserem Beispiel ist die Substitutionselastizität $\sigma=0,2$. $\sigma$ errechnet sich bei unserer CES-Nutzenfunktion folgendermaßen, vgl. Henderson/Quandt [(1977,s. 78)].

$$
\sigma=\frac{1}{1+4}=\frac{1}{5}=0,2 \text {. }
$$

Ist $z \cdot B$. die Nutzenfunktion gegeben durch

$u(x, y, n)=x^{\frac{1}{2}}+\frac{1}{2} y^{\frac{1}{2}}$ und $f(n)=n^{\frac{1}{2}}$

dann ist $\sigma=2$ und die $v(n)$-Funktion

$v(n)=2^{-\frac{1}{2}}\left(n^{-\frac{1}{4}}+\frac{1}{2} n^{\frac{1}{4}}\right)$

hat bei $n=4$ ein Minimum, so daß sie folgende Form hat:

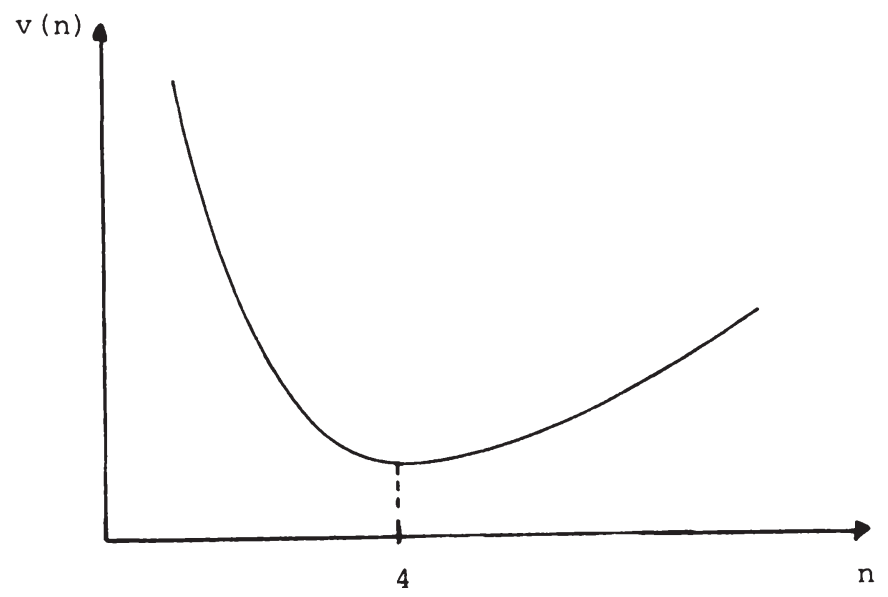


Die Produktionsfunktion sei gegeben durch
(A. 2) $\quad f(n)=8 n \frac{1}{2} \quad b z w$.

d.h. die Budgetrestriktion lautet

(A. 3) $n x+y=8 n^{\frac{1}{2}}$

Der Nutzen des repräsentativen Individuums wird maximiert unter Berücksichtigung der Budgetrestriktion:
(A. 4) $L=u(x, y)+\lambda(f(n)-n x-y)$

woraus man folgende Bedingung erhält:
(A. 5) $n \frac{\mathrm{u}_{2}}{\mathrm{u}_{1}}=1$
(Samuelson-Bedingung)

sowie die Nebenbedingung

(A. 6) $\mathrm{nx}+\mathrm{y}=\mathrm{f}(\mathrm{n})$

Aus diesen beiden Gleichungen kann $x$ und $y$ in Abhängigkeit von $n$ errechnet werden, so daß man die indirekte Nutzenfunktion $v(n)$ erhält:

(A. 7) $\quad v(n)=u(x(n), y(n))$.

$\mathrm{V}$ (n) wird maximiert.

Für obiges Beispiel erhält man

$x=8 n^{\frac{3}{10}}\left(n^{\frac{4}{5}}+2^{\frac{8}{5}}\right)^{-1}$ und $y=2^{\frac{23}{5}} n^{\frac{1}{2}}\left(n^{\frac{4}{5}}+2^{\frac{8}{5}}\right)^{-1}$

Für die $v(n)$-Funktion gilt dann

(A. 8) $v(n)=257^{\frac{1}{4}} \cdot 2^{\frac{13}{5}} n^{\frac{1}{2}}\left(n^{\frac{4}{5}}+2^{\frac{8}{5}}\right)^{-\frac{5}{4}}$

$\mathrm{v}(\mathrm{n})$ hat ein Maximum, wenn gilt $\mathrm{v}^{\prime}(\mathrm{n})=0$ und $\left.\mathrm{v}^{\prime \prime}(\mathrm{n})\right|_{\mathrm{V}^{\prime}(\mathrm{n})=0}<$. 
(A. 9) $v^{\prime}(n)=257^{\frac{1}{4}} \cdot 2^{\frac{13}{5}}\left(n^{\frac{4}{5}}+2^{\frac{8}{5}}\right)^{-\frac{9}{4}}\left(2^{\frac{7}{5}} n^{-\frac{1}{2}}-\frac{1}{2} n^{\frac{3}{10}}\right) \stackrel{!}{=} 0$ Die Gleichung $V^{\prime}(n)=0$ ist für $n=4$ erfüllt.

$\left.v^{\prime \prime}(n)\right|_{v^{\prime}(n)=0}=257^{\frac{1}{4}} 2^{\frac{13}{5}}\left(n^{\frac{4}{5}}+2^{\frac{8}{5}}\right)^{-\frac{9}{4}}\left(-2^{\frac{6}{5}} n^{-\frac{3}{2}}-\frac{3}{20} n^{-\frac{7}{10}}\right)<0$

Daraus folgt, $v(n)$ hat bei $n=4$ ein Maximum, wobei $x=2$ und $y=8$ ist. $v(n)$ hat dann folgende Form

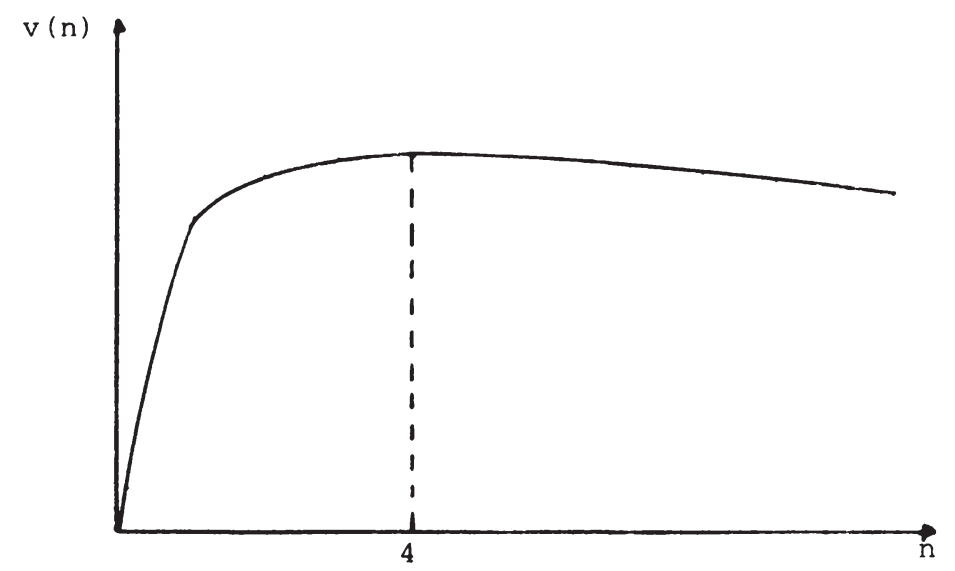

Schaubild A.1

Eine andere Darstellungsform ist diejenige im $(x, y)$-Diagramm.

Dazu muß zuerst die Transformationskurve zwischen $x$ und $y$ ausgerechnet werden. Es gilt (vgl. Stiglitz (1977)).

$f(n)=x n+y$, womit $x=\frac{f(n)-y}{n}$

Für beliebiges $y$ wird $x$ über $n$ maximiert, so daß

$\frac{d x}{d n}=\frac{f^{\prime} n-(f-y)}{n^{2}}=0^{\circ}$

und

$\frac{d^{2} x}{d n^{2}}=\frac{\left(f^{\prime \prime} n+f^{\prime}-f^{\prime}\right) n^{2}-\left(f^{\prime} n-(f-y)\right) 2 n}{n^{4}}$ 
$\left.\frac{\mathrm{d}^{2} \mathrm{x}}{\mathrm{dn} 2}\right|_{\frac{\mathrm{dx}}{\mathrm{dn}}=0}=\frac{\mathrm{f}^{\prime \prime}}{\mathrm{n}}<0 \quad \Rightarrow \operatorname{Max}$

Man erhält dann

$$
\begin{aligned}
& y=f-f^{\prime} n \\
& x=f^{\prime}
\end{aligned}
$$

Aus diesen beiden Gleichungen erhält man die konvexe Transformationskurve zwischen $x$ und $y$, wobei jeder Punkt auf dieser Kurve ein unterschiedliches $n$ symbolisiert. Für unser Beispiel ergibt sich also

$$
x=4 n^{-\frac{1}{2}}, y=4 n^{\frac{1}{2}}
$$

unci damit

$$
x=\frac{16}{y}
$$

Die Indifferenzkurven aus unserer CES-Nutzenfunktion sind dann

$$
x=\left[\frac{257}{\bar{u}^{4}}-\frac{256}{y^{4}}\right]^{-\frac{1}{4} \cdot}
$$

Wo sich die beiden Kurven tangieren, liegt der Optimalpunkt. D.h. es muß gelten.

$$
\left.\frac{d x}{d y}\right|_{T K}=\left.\frac{d x}{d y}\right|_{I K}
$$

Durch Ausführung dieser Rechenoperation und Einsetzen erhält 
man $x=2$ und $y=8$ sowie $u=2056^{\frac{1}{4}}$ und damit auch $n=4$. 20) Dieser Sachverhalt ist in Schaubild A.2 dargestellt.

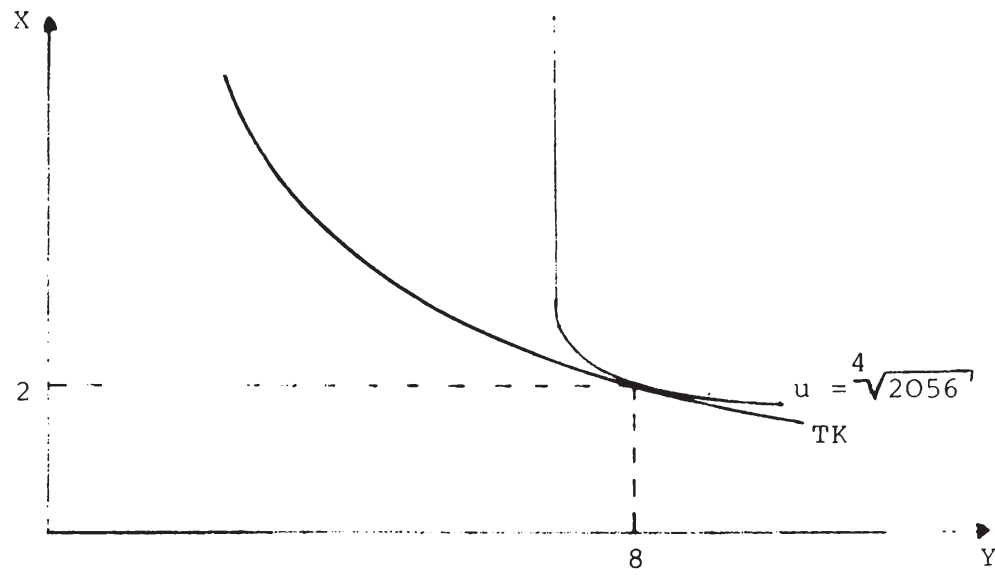

Schaubild A. 2

20) Für das Beispiel aus Fußnote 19, S.140, erhält man für die Transformationskurve $\mathrm{x}=\frac{1}{4 y}$ und für die Indifferenzkurve $x=\left(\bar{u}-\frac{1}{2} y^{\frac{1}{2}}\right)^{2}$ folgt, daß für $x=\frac{1}{4}$ und $y=1 \quad(d \cdot h . n=4)$ der Nutzen maximal ist.

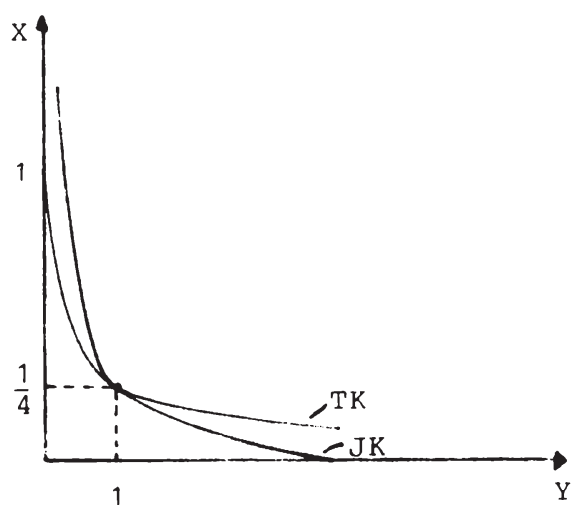


Literaturverzeichnis:

Breton, A. 1970, "Public Goods and the Stability of Federalism", in: Kyklos, Vol. 23, 882-902.

Buchanan, J.M. 1965, "An Economic Theory of Clubs", in: Economica, Vol. 32, 1-14.

Buchanan, J.M. und Goetz, C. 1972, "Efficiency Limits of Fiscal Mobility: An Assessment of the Tiebout Model", in: Journal of Public Economics, I, 25-45.

Buchanan, J.M. und Wagner, R.E. 1970, "An Efficiency Basis for Federal Fiscal Equalization", in: Margolis, J. (Ed.) 1970, The Analysis of Public Output, Baltimore, Resources for the Future, Inc., The John Hopkins Press.

Fischel, W.A. 1980, "Externalities and Zoning", in: Public Choice, Vol. 35, 37-43.

Flatters, F., Henderson, V., Mieszkowski, P. 1974, "Public Goods, Efficiency, and Regional Fiscal Equalization", in: Journal of Public Economics, Vol. 3, 99-112.

Henderson, J.M. und Quandt, R.E. 1977, Mikroökonomische Theorie, München: Vahlen.

IIirschmann, A.O. 1970, Exit, Voice and Loyalty, Cambridge: Harvard University Press.

Loehr, W. 1974, "Fiscal Federalism, Spillovers and the Export of Taxes: An Extension", in: Kyklos, Vol. 27. 606-609.

Milleron, J.C. 1977, Diskussion des Aufsatzes von Stiglitz, J.E., in: Feldstein, M.S. und Inman, R.P. (Ed.), The Economics of Public Service, London, The MacMillan Press Ltd., 493-501.

Nueller, D.C. 1979, Public Choice, Cambridge u.a., Cambridge University Press.

Musgrave, R.A. 1939, "The Voluntary Exchange Theory of Public Economy", in: Quarterly Journal of Economics, 213-217.

Musgrave, R.A. 1955, "A Multiple Theory of the Budget", paper read at the Econometric Society annual meeting.

Pestieau, P. 1979, "Fiscal Mobility and Local Public Goods: A Survey of the Empirical and Theoretical studies of the Tiebout Model", unveröffentlicht.

samuelson, P.A. 1954, "The Pure Theory of Public Expenditures", Review of Economics and Statistics, Vol. 36, 387-389.

Samuelson, P.A. 1969, "Pure Theory of Public Expenditure Theories and Taxation", in: Public Economics, Proceedings of a I.E.A. conference (eds. Margolis, J. and Guitton, H.), MacMillan, London, 98-123.

Sandler, T. 1975, "Pareto Optimality, Pure Public Goods, Impure Public Goods and Multiregional Spillovers", in: Scottish Journal of Political Economy, Vol. 22, 25-38.

Sandler, T. und Shelton, R.B. 1972, "Fiscal Federalism, Spillovers and the Export of Taxes", in: Kyklos, Vol. 25, 736-753.

Sandler, T. und Tschirhart J.T. 1980," The Economic Theory of Clubs. An Evaluative Survey", Journal of Economic Literature, Vol. 18, 1481-1521. 
Stiglitz, J.E. 1977, "The Theory of Local Public Goods", in: Feldstein, M.S. und Inman, R.P. (Ed.), The Economics of Public Services, London, The MacMillan Press Ltd., 274-333.

Tiebout, C.M. 1956, "A Pure Theory of Local Public Goods", in: Journal of Political Economy, Vol. 64, 416-424.

Westhoff, F. 1977, "Existence of Equilibria in Economics with a Local Public Good", in: Journal of Economic Theory, Vol. 14, 84-112.

Wheaton, W.C. 1975, "Consumer Mobility and Community Tax Bases", in: Journal of Public Economics, Vol. 4, 377-384.

Wooders, M. 1978, "Equilibria, the Core, and Jurisdiction Structures in Economics with a Local Public Good", in: Journal of Economic Theory, Vol. 18, 328-348.

Wooders, M. 1980, "The Tiebout Hypotheses: Near Optimality in Local Public Good Economies", in: Econometrica, Vol. 48, 1467-1485. 
Mehrheitswahl als Instrument regionalisierter Umweltpolitik

- Eine allgemeine Gleichgewichtsanalyse -

\author{
F. Dudenhöffer
}

\title{
1. Einleitung
}

Bei Konsum- und Produktionsaktivitäten entstehen Kuppelprodukte, die bei gegebenem stand der Technik keine weitere Verwendung finden. Diese Abfallprodukte - oder Emissionen - verursachen bei ihrer Abgabe an Umweltmedien Umweltqualitätsbeeinträchtigungen. Unterstellt man, daß sich kein Individuum vom "Konsum" vorhandener Umweltbeeinträchtigungen ausschließen kann, liegt ein Problem der Allokation eines öffentlichen Gutes vor. Eine regionale Dimension eines derartigen Umweltallokationsproblems entsteht in einem Mehrregionensystem durch die Existenz von Umweltinterdependenzen zwischen den einzelnen Regionen, so daß Kuppelprodukte, die bei ökonomischen Aktivitäten in einer Region anfallen und dort an die Umwelt abgegeben werden über Diffusionsvorgänge zu Umweltbelastungen in anderen Regionen führen.

Dieser Beitrag wurde im Rahmen des Sonderforschungsbereiches 5 "Staatliche Allokationspolitik im marktwirtschaftlichen System" erstellt. Für sehr wertvolle Hinweise und Anregungen danke ich Herrn Prof. Dr. R. Pethig (Oldenburg) und Herrn Prof. Dr. H. Siebert (Mannheim). Weiteren Dank schulde ich meinen Kollegen am Lehrstuhl für Volkswirtschaftslehre und AuBenwirtschaft der Universität Mannheim. 
Etabliert man eine zentrale Umweltbehörde, kann gezeigt werden, daß über eine Besteuerung der Emissionen gemäß Pigou'scher Vorschrift eine Lösung des regionalisierten Umweltallokationsproblem gefunden werden kann, die das Pareto-Kriterium erfüllt. 1) Dabei wird vorausgesetzt, daß die Umweltbehörde vollständige Information über die Charakteristiken der Wirtschaftssubjekte besitzt und das ökologische system der ökonomie kennt. Neben diesen sehr strengen Voraussetzungen bezüglich des Informationsbeschaffungs- und verarbeitungssystems, gibt die Pigou'sche Steuerlösung das bei der Allokation öffentlicher Güter auftretende Freifahrerproblem als gelöst vor und vernachlässigt den "Ressourcenverzehr" der Umweltbehörde bei Informationsbeschaffungs- und verarbeitungsaktivitäten.

Baumol/Oates (1971) schlugen deshalb als Lösungskonzept der eben skizzierten Probleme die sogenannten preis- und Standardsysteme vor. Bei diesen systemen wird die anzustrebende Umweltqualität als "fixed-target" vorgegeben und die diesem "fixedtarget" zugeordneten preise als Emissionssteuer interpretiert. ${ }^{2}$ ) Die Bestimmung dieser standards erfolgt dabei weitgehend in einem institutionellen Vakuum, womit keine Rückkopplung zwischen ökonomischen Variablen und standardbestimmung stattfindet.

Der im folgenden zu beschreibende Mehrheitswahlmechanismus zur Allokation des öffentlichen Gutes "Umweltqualität" kann als eine Art modellendogenes Preis-Standardsystem interpretiert werden. Argumentiert wird in einem Zwei Regionenmodell, wobei zwischen beiden Regionen einseitige interregionale Schadstoffdiffusionsprozesse unterstellt wurden. Die standardbestimmung für eine Region erfolgt dabei über eine Entscheidung durch Mehrheitswahl,

1) Vgl. dazu u.a. Siebert (1975), Siebert (1978a), Siebert (1978b), Siebert (1981), Tietenberg (1974).

2) Eine andere Variante des Preis-Standard-Ansatzes interpretiert die einem Standard zugeordneten Preise als Marktpreise aus dem Verkauf der in der Höhe des Standards angebotenen Emissionszertifikate. 
indem jedes stimmberechtigte Individuum dieser Region - jeder Konsument - den von ihm angestrebten Umweltqualitätsstandard bzw. die "gewünschte" Immissionsmenge - in geheimer Wahl einem Wahlleiter bekanntgibt und dieser aus der Menge mitgeteilter "Immissionswünsche" das "Mehrheitsumweltbelastungsniveau" ermittelt. Damit ist ein dezentraler, nicht-diktatorieller, anonymer Allokationsmechanismus definiert. Analog zum Markt, der dezentrale Informationen einzelner Teilnehmer gewissermaßen über Preisregulationen "zentriert", erfolgt beim Mehrheitswahlmechanismus eine Verarbeitung der einzelnen dezentralen Informationen, indem die Information des Medianwählers selektiert wird. Es liegt ein "positiver" Politikansatz vor, der sich bezüglich der Informationsbeschaffung und Informationsverarbeitung durch seine "bestechende" Einfachheit gegenüber den o.a. "normativen" Ansätzen auszeichnet. ${ }^{3)}$

Die Analyse von Mehrheitswahlentscheidungen im Rahmen der Ressourcenallokation kann bis Bowen (1943) zurückverfolgt werden. A. Klevorick und G. Kramer (1972) untersuchten in ihrem Artikel "Social Choice on Pollution Management: The Genossenschaften" in einem Partialmodell die Frage der Existenz von Abstimmungsgleichgewichten. Der Abstimmungsgegenstand bildet dabei der Emissionsteuersatz der ökonomie, wobei ein direkter zusammenhang zwischen Steuersatz und Immissionsniveau unterstellt wird. Interessant am Ansatz von Klevorick und Kramer ist die Einbeziehung von Firmen in den EntscheidungsfindungsprozeB zur Emissionsteuerermittlung. Im Klevorick-Kramer-Modell liegt keine feste stimmausstattung eines wählers vor, sondern das "Stimmgewicht" der einzelnen Wähler wird modellendogen, in Abhängigkeit von den Emissionen der Individuen bestimmt. Kurioserweise erhalten damit die größten "Umweltsünder" die meisten Stimmen zur Bestimmung der Umweltqualität. Wie der Titel der Arbeit von Klevorick und Kramer erkennen läßt, stellt ihre Analyse die theoretische Aufarbei-

3) Die "Positivität" des Ansatzes beinhaltet allerdings das Werturteil, daß jedes Individuum mit einem à priori gegebenen Anteil der Gesamtstimmen bzw. jedes Individuum mit genau einer Stimme ausgestattet ist. 
tung der Funktionsweise der in der BRD betriebenen Wassergenossenschaften an der Ruhr dar.

Slutsky (1977) integrierte den Mehrheitswahlmechanismus zur Allokation öffentlicher Güter in ein allgemeines Gleichgewichtsmodell mit öffentlichen und privaten Gütern und zeigte die Existenz eines "General Voting Equilibrium".

Im folgenden Abschnitt 2 dieses Beitrages wird zunächst eine allgemeine Charakterisierung von Wahl-oder Abstimmungsgleichgewichten gegeben. Daran anschließend wird in Abschnitt 3 die Modellökonomie spezifiziert und die Beschränktheit der Menge erreichbarer Allokationen der Modellökonomie gezeigt. In Abschnitt 4 wird der Allokationsmechanismus der Modellökonomie spezifiziert und die Existenz eines kombinierten Markt- und Abstimmungsgleichgewichts in einem Anhang zu Abschnitt 4 gezeigt.

\section{Das Mehrheitswahlgleichgewicht}

Wir gehen von einer Anzahl von $\mathrm{n}$ Individuen aus, die aus einer ihr vorgegebenen Menge von Alternativen $\mathrm{Z}$ - dem Alternativenoder Politikraum - eine Auswahl treffen soll. Eine Mehrheitswahlregel ist eine ganze $\mathrm{Zahl} d$, welche die kleinste $\mathrm{zahl}$ von Individuen angibt, die die Auswahl einer bestimmten Alternative $z \in z$ verhindern kann. Eine Alternative $\hat{z} \in Z$ ist ein Mehrheitswahlgleichgewicht unter einer Mehrheitswahlregel d - im folgenden auch d-Mehrheitswahlgleichgewicht genannt -, wenn es keine Alternative $z \in \mathrm{z}$ gibt, die von mindestens d-Individuen gegenüber der Alternative $\hat{z}$ präferiert wird. Definiert man $N=\{1, \ldots, n\}$ als die Indexmenge der Individuen und geht man davon aus, da $\beta$ für jedes Individuum $i \in N$ eine Nutzenfunktion $u_{i}$ über der Alternativenmenge definiert ist, so präferiert $i \in N$ genau dann $z \in z$ gegenüber $z^{\prime} \in z$, wenn $u_{i}(z)>u_{i}\left(z^{\prime}\right)$ gilt. Bezeichnet $p\left(z^{\prime}, z\right)=$ $\#\left\{i \in N \mid u_{i}(z)>u_{i}\left(z^{\prime}\right)\right\}$ die Anzahl der Individuen, die Alternative $z$ gegenüber Alternative z' präferieren kann ein d-Mehrheitswahlgleichgewicht formal beschrieben werden durch ${ }^{4}$ )

4) Sel A eine Menge, so wird mit\#A die Mächtigkeit der Menge A bezeichnet. 
Definition 1 :

Eine Alternative $\hat{z} \in \mathrm{z}$ heiBt d-Mehrheitswahlgleichgewicht, wenn. gilt $\{\mathrm{z} \in \mathrm{z} \mid \mathrm{p}(\hat{\mathrm{z}}, \mathrm{z}) \geqq \mathrm{d}\}=\phi$.

Die Definition 1 ist äquivalent zur Aussage, daß ein d-Mehrheitswahlgleichgewicht genau dann vorliegt, wenn beim paarweisen Abstimmungsvergleich mit jeder anderen Alternative des Politikraums, jede andere Alternative von weniger als d-Individuen präferiert wird. Daher erfüllt ein d-Mehrheitswahlgleichgewicht $\hat{z}$ für jedes $z \in Z$ die Bedingung $p(\hat{z}, z)<d$. Wählt man die ganze Zahl $d$ als $d>n / 2 \geqq(d-1)$, liegt die einfache Mehrheitswahlregel vor, womit eine Alternative $\hat{z} \in Z$ genau dann ein "einfaches" Mehrheitswahlgleichgewicht - oder kurz Mehrheitswahlgleichgewicht - ist, wenn $p(\hat{z}, z) \leqq n / 2$ für jedes $z \in z$ erfüllt ist. Für den Fall der einfachen Mehrheitswahlregel kann die Existenz multipler Mehrheitswahlgleichgewichte als auch die Nichtexistenz von Mehrheitswahlgleichgewichten anhand folgender Beispiele gezeigt werden. In dem durch Schaubild 1 illustrierten Fall wird von $n=4$ Wählern und dem Politikraum $z=\{z \in R$ $\mathrm{a} \leqq \mathrm{z} \leqq \mathrm{g}\}$ ausgegangen.

$u_{i}(z)$

Schaubild 1

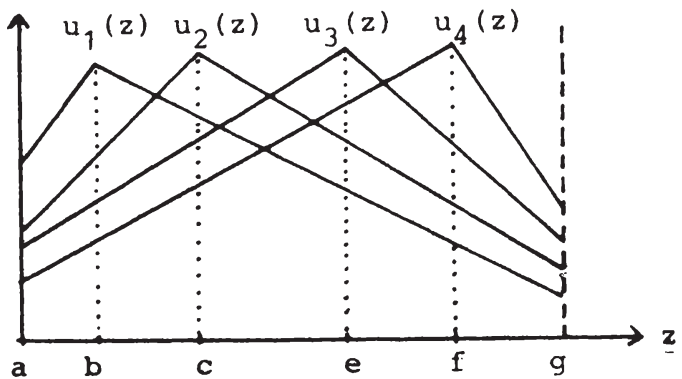

Offensichtlich ist für jedes $c \leqq \hat{z} \leqq e$ die Bedingung $\{z \in z \mid p(\hat{z}, z)$ $\geqq 3\}=\phi$ erfüllt, d.h. für jedes $z \in z$ ist $p(\hat{z}, z) \leqq n / 2=2$ erfüllt, wenn $c \leqq \hat{z}=e$ gilt. Da für jedes $z \in z$, das nicht im geschlossenen Intervall [c,e] liegt, die eben genannte Bedingung nicht erfüllt ist, ist die Menge der einfachen Mehrheitswahlgleichgewichte in diesem Beispiel das Interval [c,e]. Betrachten wir jetzt die Menge aller $d=4$-Mehrheitswahlgleichge- 
wichte des Beispiels des Schaubildes 1. Diese Menge fällt mit dem Intervall $[b, f]$ zusammen. Offensichtlich ist daher jedes dMehrheitswahlgleichgewicht ein $(d+1)$-Mehrheitswahlgleichgewicht. Im obigen Beispiel kann also bei einfacher oder d=3-Mehrheitswahlregel die Auswahl einer bestimmten Alternative aus dem Politikraum verhindert werden, wenn mindestens 3 wähler gegen diese Alternative stimmen. Bei einer $d=4$-Mehrheitsregel wäre dies nur dann der Fall, wenn mindestens 4 wähler gegen diese Alternative stimmen.

In dem in Schaubild 2 dargestellten Fall wird von einem politikraum, der aus genau 3 Alternativen, nämlich a,b und c besteht, ausgegangen.

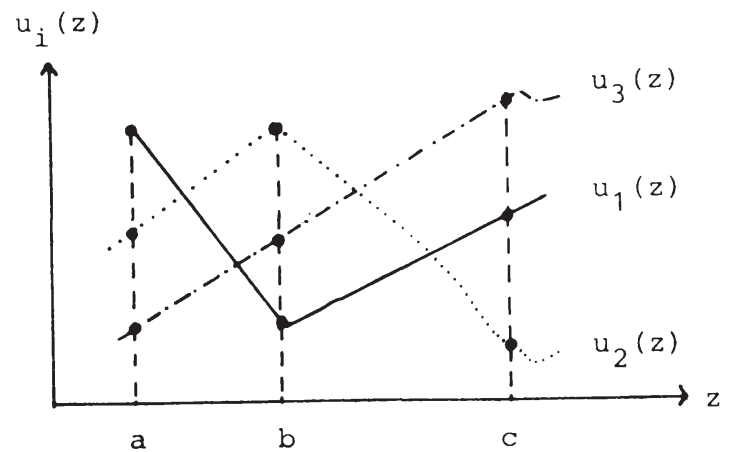

Schaubild 2

Man erkennt hierbei, $d a \beta p(a, b)=2, p(b, c)=2$ und $p(c, a)=2$ gilt, womit für jede Alternative $z^{\prime} \in\{a, b, c\}$ gilt $\left\{z \in z \mid p\left(z^{\prime}, z\right)\right.$ $\geqq 2\} \neq \phi$ und gemäß Definition 1 kein Mehrheitswahlgleichgewicht existiert. In dem durch Schaubild 3 illustrierten Fall ist ein zweidimensionaler Politikraum $\mathrm{z} \subset \mathrm{R}^{2}$ unterstellt. ${ }^{5)} \mathrm{z}$ wird dabei gewählt als die Menge aller punkte im Inneren und auf dem Rand des gleichseitigen Dreiecks ABC.

Desweiteren wird davon ausgegangen, daß $\mathrm{n}=3$ Wähler vorhanden sind, wobei die präferenzen des wähler 1 durch Kreise um punkt A,

5) Ein dem nachstehenden Beispiel ähnlicher Fall diskutiert Greenberg (1980), S. 633 . 


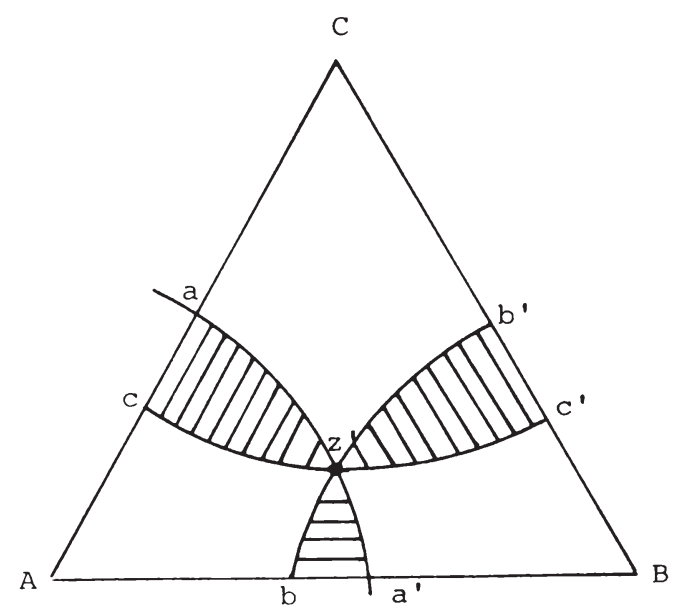

Schaubild 3

diejenigen des Wählers 2 durch Kreise um Punkt B und diejenigen des Wählers 3 durch Kreise um Punkt $C$ abgebildet werden. So ist Wähler 1 gegenüber allen Alternativen des Poltikraumes, die auf dem Kreissegment $a z^{\prime} a^{\prime}$ liegen indifferent und präferiert einen Punkt $z \in Z$ gegenüber $z^{\prime}$, wenn der Abstand des Punktes $z$ zum Punkt A kleiner ist als der Abstand zwischen $z^{\prime}$ und A. Entsprechendes gilt für die wähler 2 und 3. Aus Schaubild 3 wird deutlich, daß für jeden punkt $z$, der im Inneren einer der schraffiert ge- . zeichneten Mengen liegt, die Bedingung gilt: $p\left(z^{\prime}, z\right)=2$. Daher ist Punkt $z^{\prime}$ kein Mehrheitswahlgleichgewicht. Da man für jedes $z \in Z$ derartige "schraffierte" Mengen finden kann, existiert für dieses Beispiel kein einfaches oder, in Worten der Definition 1, kein $\mathrm{d}=2$-Mehrheitswahlgleichgewicht. Andererseits wird aus den eben gemachten Ausführungen deutlich, daß ein $d=3-$ Mehrheitswahlgleichgewicht für dieses Beispiel existiert. Da für jeden Punkt $\hat{z} \in z$ gilt, $\operatorname{da} \beta \mathrm{p}(\hat{z}, z) \leq 2$ für jedes $z \in z$ erfüllt wird, erhalten wir die Menge aller $d=3$-Mehrheitswahlgleichgewichte im vorliegenden Beispiel als die Menge $Z$.

Die vorgestellten Fälle lassen vermuten, daß einfache Mehrheits- 
gleichgewichte bei quasi-konkaven Nutzenfunktionen und eindimensionalen Politikräumen existieren, wohingegen im Falle mehrdimensionaler Politikräume bei geeigneter Wahl einer ganzen $\mathrm{Zahl} d$ entsprechende d-Mehrheitswahlgleichgewichte existieren. Diese Vermutungen werden präzisiert und bestätigt durch

Lemma 1 ( $\mathrm{J}$. Greenberg) :

Sei der Politikraum $\mathrm{z}$ eine nichtleere, kompakte und konvexe Teilmenge des $R^{m}$ mit der Dimension $m$ und die Präferenzen jedes Individuums $i \in N$ über dem Politikraum durch stetige quasikonkave Nutzenfunktionen abbildbar. Unter diesen Voraussetzungen existiert für $d>(m /(m+1))$ n ein d-Mehrheitswahlgleichgewicht. Wenn $\mathrm{d} \leqq(\mathrm{m} /(\mathrm{m}+1)) \mathrm{n}$ gilt, gibt es $\mathrm{n}$ stetige, streng quasi konkave Nutzenfunktionen derart, daß kein d-Mehrheitswahlgleichgewicht existiert.

\section{Beweis :}

Greenberg (1980), Theorem 1 und Theorem 2 .

Der obige Hilfssatz verallgemeinert das Ergebnis von Black (1958), wonach bei eingipfligen Präferenzen über kompakten, konvexen eindimensionalen Politikräumen einfache Mehrheitswahlgleichgewichte existieren. Interessant am obigen Hilfssatz ist die Einbeziehung mehrdimensionaler Politikräume. Dabei gilt, daß bei steigender Dimension m des Politikraums nur noch Gleichgewichte mit größerer Zahl d existieren. Im Extremfall unendlich dimensionaler Poltikräume existieren dann lediglich n-Mehrheitswahlgleichgewichte, d.h. eine Alternative des Politikraums kann hierbei nur dann verworfen werden, wenn alle Individuen gegen diese Alternative stimmen. Das Verfahren zur Ermittlung von d-Mehrheitswahlgleichgewichten ist der paarweise Vergleich aller möglichen Alternativen des Politikraumes. Stände ein Wahlleiter vor dem Problem, unter Gültigkeit der Voraussetzungen des obigen Hilfssatzes ein entsprechendes d-Mehrheitswahlgleichgewicht zu ermitteln, wären bei dieser Wahl unendlich viele Abstimmungsgänge notwendig. Dieses komplizierte Verfahren der Ermittlung 
von Wahlsiegern kann wesentlich vereinfacht werden, wenn eindimensionale Politikräume vorliegen. Unter dieser Voraussetzung ist die Menge der einfachen Mehrheitswahlgleichgewichte gleich der Medianmenge der: individuell-besten Alternativen. Daher kann in diesen Fällen der Wahlleiter bei der Ermittlung von Mehrheitswahlgleichgewichten derart verfahren, daß jedem Individuum ein "Stimmzettel" zugeteilt wird, auf dem es die individuell-beste Alternative vermerkt und der Wahlleiter das Wahlergebnis über die Berechnung des Medians der abgegebenen Vorschläge bestimmt. Sei eine Indexinenge $N=\{1, \ldots, n\}$ mit $n=\# N$ und für jedes $i \in N$ ein Element $\hat{z}_{i} \in \mathrm{Z} \subset \mathrm{R}$ definiert, so wird im folgenden die Medianmenge definiert durch

$$
\begin{gathered}
\operatorname{median} \hat{z}_{i}=\left\{z \in z | \# \{ i \in N | \hat { z } _ { i } \geqq z \} \geqq n / 2 \text { und } \# \left\{i \in N \mid \hat{z}_{i}\right.\right. \\
i \in N\} \geqq n / 2\}
\end{gathered}
$$

Zusammenfassend läßt sich dann sagen

Lemma 2 :

Sei $\mathrm{Z}$ eine nichtleere, kompakte und konvexe Teilmenge des $\mathrm{R}$ und für jedes $i \in N$ die Funktion $u_{i}: z \rightarrow R$ streng quasi-konkav. Unter diesen Voraussetzungen ist $\hat{z} \in \mathrm{Z}$ genau dann ein Mehrheitswahlgleichgewicht, wenn gilt

$$
\hat{z} \in \operatorname{median} \hat{z}_{i}
$$

$i \in N$

wobei für jede!s $i \in N$ gilt: $u_{i}\left(\hat{z}_{i}\right)=\max u_{i}(z)$

$$
z \in \mathrm{Z}
$$

\section{Beweis :}

(i) Für jedes $i \in N$ ist die Menge $P_{i}(\hat{z}) \equiv\left\{z \in z \mid u_{i}(z)>u_{i}(\hat{z})\right\}$ konvex: Sei $z^{\prime}, z^{\prime \prime} \in P_{i}(\hat{z})$ und $0<\lambda<\hat{\imath}$. Da die Funktion $u_{i}$ streng quasi-konkav j.st, gilt $u_{i}\left(\lambda z^{\prime}+(1-\lambda) z^{\prime \prime}\right)>\min \left[u_{i}\left(z^{\prime}\right), u_{i}\left(z^{\prime \prime}\right)\right]$ und wegen der Definition von $P_{i}(\hat{z})$ folgt $\left(\lambda z^{\prime}+(1-\lambda) z^{\prime \prime}\right) \in P_{i}(\hat{z})$.

(ii) Da $z$ konvex, kompakt und nichtleer ist, $u_{i}$ eine streng quasi-konkave Funktion ist, existiert für jedes $i \in N$ genau ein $\hat{z}_{i}$, womit median $\hat{z}_{i \in N}$ eine nichtleere Menge ist. Da 
$\#\left\{i \in N \mid \hat{z}_{i} \geqq \hat{z}\right\}+\#\left\{i \in N \mid \hat{z}_{i}<\hat{z}\right\}=n$ gilt, ist wegen der Definition der Medianmenge die Aussage $\hat{z} \in \underset{i \in N}{\operatorname{median}} \hat{z}_{i}$ äquivalent zu

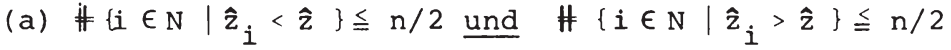

Da $P_{i}(\hat{z})$ konvex ist, gilt $f$ ür jedes $i \in N$ entweder $P_{i}(\hat{z}) \subset A$ oder $\mathrm{P}_{i}(\hat{z}) \subset \mathrm{B}$ oder $\mathrm{P}_{i}(\hat{z})=\phi$, wobei $\mathrm{A} \equiv\{z \in \mathrm{z} \mid \mathrm{z}<\hat{z}\}$ und $\mathrm{B} \equiv$ $\{z \in z \mid z>\hat{z}$ \}. Die Aussage (a) ist deshalb äquivalent zur Aussage

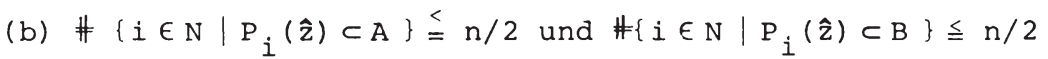

Da definitionsgemäß $\hat{z} \notin P_{i}(\hat{z})$ gilt, impliziert die Aussage (b)

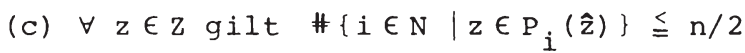

und wegen der Konvexität der $\mathrm{P}_{i}(\hat{z})$ wird (b) von (c) impliziert. Q.E.D.

\section{Die Modellökonomie}

Nach diesen vorbereitenden Ausführungen zum Mehrheitswahlmechanismus kann nunmehr das Grundmodell der ökonomie beschrieben werden, welches den Rahmen für die Analyse des Allokationsinstrumentes der Mehrheitswahl absteckt. Es wird eine ökonomie unterstellt, die aus zwei Regionen besteht und interregionale Verflechtungen im Güter- und Umweltbereich aufweist. In der Ökonomie gibt es $(n+4)$ Güter, die vorwiegend durch $1=1, \ldots$, $n+4$ indiziert werden. Die Güter $1=1, \ldots, n+2$ stellen private Güter dar, wobei die ersten $n$ Gütertypen interregional vollständig mobil sind. Das $(n+1)$-te bzw. $(n+2)$-te Gut ist ein Kuppelprodukt, das bei produktionsaktivitäten in Region 1 bzw. 2 anfällt. Da die einzige Verwendung dieser Kuppelprodukte ihre Abgabe an die Umwelt ist, werden sie als Emissionen bezeichnet. Die Güter $(n+3)$ und $(n+4)$ sind die Umweltbelastungsindikatoren der Region 1 bzw. 2 und werden auch regionale Immissionsniveaus genannt. Es gibt $(\mathrm{K}+1)$ Produzenten, wovon $\mathrm{K}_{1}$ in Region 1 und $\left(\mathrm{K}-\mathrm{K}_{1}\right)$ in Region 2 angesiedelt sind. Ein Produzent $\mathrm{j}$ wird vollständig durch seinen produktionsraum $\mathrm{Y}^{\mathrm{j}}$ beschrieben. Für $\mathrm{j}=1, \ldots, \mathrm{K}$ gilt dabei 


$$
\begin{aligned}
& y^{j} \equiv\left\{y^{j} \mid y^{j} \equiv\left(y_{1}^{j}, \ldots, y_{n+2}^{j}\right),\left(y_{n+1}^{j}, y_{n+2}^{j}\right) \in R_{+^{\prime}}^{2}, y_{n+1}^{j}=o\right. \text { für } \\
& \left.\quad\left[1=2 \text { and } j=1, \ldots, K_{1}\right] \text { oder }\left[1=1 \text { und } j=k_{1}+1, \ldots, k\right]\right\} \subset R^{n+2}
\end{aligned}
$$

Für den Fall, daß Gut 1 bei der Produktion $y^{j} \in Y^{j}$ ein Inputgut ist, wird dies durch $\mathrm{Y}_{l}^{j}<0$ angezeigt. Wie aus der Definition des Produktionsraums erkennbar, ist unterstellt, daß ein produzent die bei Produktionsprozessen anfallenden Kuppelprodukte nur in der Region an die Umwelt abgeben kann, in welcher sich seine produktionsstätten befinden. Da jede produktion nur Kombinationen der ersten $(n+2)$-Güter umfaßt, ist von Rückwirkungen von Umweltzuständen auf die Produktionssektoren abstrahiert. Emissionen fallen bei der produktion privater Güter als "variable Kuppelprodukte" an. 6) Damit ist es für einen Produzenten möglich, über Inputvariationen die Entstehung der Kuppelproduktion "einzuschränken", ohne die Ausbringung der anderen "Outputgüter" zu verändern. Für den Fall, daß $y^{j}$ eine geplante produktion des j-ten Produzenten ist, wird der Vektor $\left(y_{n+1}, y_{n+2}\right)$ mit $y_{n+1}=$ $\stackrel{K}{N}=1_{\mathrm{K}}^{\mathrm{K}} \mathrm{Y}_{\mathrm{n}+1}^{j}$ für $1=1,2$ als gesamtwirtschaftliches Emissionsangebot bezeichnet. Die Produktionsmengen $Y^{j}$ der Produzenten $j=1, \ldots, k$ sollen die folgenden Standardannahmen der allgemeinen Gleichgewichtstheorie erfüllen.

Annahme (a)

(a.1) Möglichkeit der Nullproduktion $o \in Y^{j}$ für $j=1, \ldots, k$

(a.2) Stetigkeit und Konvexität $\mathrm{Y}^{j}$ ist abgeschlossen und konvex für $\mathrm{j}=1, \ldots, \mathrm{K}$

(a.3) Unmöglichkeit freier produktion $Y \cap R_{+}^{n+2} \subset\{0\} \quad$ mit $Y \equiv \sum_{j=1}^{K} Y^{j}$

(a.4) Irreversibilität $\mathrm{Y} \cap(-\mathrm{Y}) \subset\{\mathrm{O}\}$

6) Der Begriff "variable Kuppelprodukte" wird in Anlehnung an R.Pethig (1979), S.19 f gebraucht. 
Der Produzent $\mathrm{K}+1$ heißt Umweltproduzent und die ihm zugeordnete Produktionsmenge $\mathrm{Y}^{\mathrm{K}+1}$ wird Umwelttechnologie genannt. ${ }^{7}$ ) Uber die Umwelttechnologie erfolgt die Beschreibung des ökosystems der ökonomie, womit durch diese "Technologie" ein Zusammenhang zwischen den an die Umwelt abgegebenen Emissionen und den Umweltbelastungs- oder Immissionsniveaus der Regionen definiert wird. Dabei gilt

Annahme (b)

$$
\begin{aligned}
\mathrm{Y}^{\mathrm{K}+1} \equiv & \left\{(e, z) \in \mathrm{R}_{+}^{\mathrm{n}+4} \mid \mathrm{e}_{1}=\ldots=\mathrm{e}_{\mathrm{n}}=0, \mathrm{z}_{1}=\mathrm{z}_{1}\left(\mathrm{e}_{\mathrm{n}+1}, \mathrm{e}_{\mathrm{n}+2}\right),\right. \\
& \mathrm{z}_{2}=\mathrm{z}_{2}\left(\mathrm{e}_{\mathrm{n}+2}\right), \mathrm{o}=\mathrm{z}_{1}(\mathrm{o}) \text { und } \mathrm{z}_{1} \text { ist eine stetige, monoton } \\
& \text { steigende, konvexe Funktion mit } 1=1,2\}
\end{aligned}
$$

Ist $(e, z)$ eine geplante Produktion des Umweltproduzenten, so wird e die gesamtwirtschaftliche Emissionsnachfrage und $z$ das gesamtwirtschaftliche Immissions- oder Umweltbelastungsangebot genannt. Aus Annahme (b) ist ersichtlich, daß einseitige interregionale Schadstoffdiffusionen unterstellt werden. Damit beeinträchtigt eine in der Region 2 emittierte Schadstoffeinheit über die "Diffusionsfunktion" $z_{1}$ und $z_{2}$ die Umwelt der Region 1 und 2, während eine in Region 1 emittierte Schadstoffeinheit nur zu Umweltbeeinträchtigungen in derselben Region führt. ${ }^{8}$ ) Ferner wird mit Annahme (b) unterstellt, daß es dem Umweltproduzenten nicht möglich ist, durch Einsatz von Faktoren Entsorgungsleistungen zu produzieren, und somit zu Umweltqualitätsverbesserungen beizutragen. Da über die Diffusionsfunktionen $\mathrm{z}_{1}, \mathrm{z}_{2}$ eine eindeutige Zuordnung zwischen Emissionen und Umweltqualitätsbeeinträchtigungen definiert ist, wird über die Umwelttechnologie das Problem der verursachungsgerechten zuordnung von Umweltqualitätsbeeinträchtigungen als gelöst vorgegeben, wenn diese technolo-

7) Vgl. dazu auch die Verwendung der Ausdrücke "Umwelttechnologie" und "Umweltproduzent" bei Pethig (1979) in einem ähnlichen Zusammenhang.

8) Vgl. dazu auch H. Siebert (1978a)S. 8 ff und insbesondere den in Kapitel 7 diskutierten "regionalen Allokationsaspekt". 
gischen Zusammenhänge hinreichend bekannt sind. Weiterhin ist aus Annahme ( $b$ ) erkennbar, daß wegen der Definition der Diffusionsfunktionen die Umwelttechnologie eine nicht konvexe Menge ist. Wären die: Emissions-Immissionszusammenhänge definiert durch $z_{1} \geqq z_{1}\left(e_{n+1}, e_{n+2}\right)$ würde gegen das Gesetz der Erhaltung der Masse verstoßen, da für jeden vorgegebenen Betrag an Emissionen "unendliche" Umweltbelastungen möglich sind oder aber für $z_{1} \leqq z_{1}\left(e_{r+1}, e_{n+2}\right)$ jedes Umweltproblem gegenstandlos.

In der ökonomie gibt es $\mathrm{N}$ Konsumenten, für die in der Regel der Index $i=1, \ldots, N$ verwendet wird. Von diesen $N$ Konsumenten sind $\mathrm{N}_{1}$ der Region 1 und $\left(\mathrm{N}-\mathrm{N}_{1}\right)$ der Region 2 zugeordnet. Ein Konsument wirç vollständig charakterisiert durch seine Konsummenge $x^{i}$, die seinen Präferenzen zuordenbare Nutzenfunktion $u_{i}$, seine Anfangsausstattung $\omega^{i}$ und seine Anteile $\Theta_{i j} \geqq 0$ an den Gewinnen aller froduzenten $j=1, \ldots, K+1$, wobe $\sum_{i=1}^{N} \Theta_{i j}=1$ für alle $j=1, \ldots, k+1$ gil.t. Die Charakteristik eines Konsumenten $\left[x^{i}, u_{i}, \omega^{i}\right.$, $\left.\left[{ }^{\Theta}{ }_{i j}\right]_{j=1}^{K+1}\right]$ soll. folgenden Kriterien genügen

Annahme (c)

(c. 1$) x^{i} \equiv\left\{\left(x^{j}, s^{i}\right) \in R_{+}^{n+4} \mid x_{n+1}^{i}=x_{n+2}^{i}=0\right\}$

(c.2) Die Nutzenfunktion $u_{i}$ ist eine über $x^{i}$ definierte, reellwertige, stetige, streng quasi-konkave Funktion mit der Eigenschaft: $u_{i}\left(\bar{x}^{i}, \bar{s}^{i}\right)>u_{i}\left(x^{i}, \bar{s}^{i}\right)$ für $\bar{x}^{i} \geq x^{i}$ und $u_{i}\left(\bar{x}^{i}, \bar{s}^{j}\right)>u\left(\frac{i}{x}, s^{i}\right)$ für $\left.\bar{s}^{i} \leq s^{i} \cdot 9\right)$

(c.3) $\omega^{i} \in R_{++}^{n}$

Für den Fall, daß $\left(\mathrm{x}^{i}, \mathrm{~s}^{i}\right) \in \mathrm{x}^{i}$ ein geplanter Konsum von Konsument $i$ ist, wird $s^{i} \in R_{+}^{2}$ seine Umweltbelastungsnachfrage genannt, wobei $s_{1}^{i}$ mit $\left(s_{1}^{i}, s_{2}^{i}\right) \equiv s^{i}$ die Nachfrage des $i$ nach Umweltbelastungen für Region 1 bezeichnet. Aus Annahme (c.1) ist erkennbar, daß wegen $x_{n+1}^{i}=x_{n+2}^{i}=0$ der Konsument die bei Produktions-

9) Im folgenden wird mit $x \geq x^{\prime}$ die Vektorrelation $x \stackrel{\geq}{=} x^{\prime}$ und $x \neq x^{\prime}$ bezeichnet, wobei $x \geqq x^{\prime}$ äquivalent $z u x_{I} \geqq x_{I^{\prime}}$ für
$l=1, \ldots, L$ ist. 
prozessen anfallenden und an die Umwelt abgegebenen Kuppelprodukte nicht direkt konsumieren kann. Desweiteren impliziert Annahme (c.1), daß bei Konsumvorgängen keine die Umwelt beeinträchtigenden Kuppelprodukte anfallen. Der zentrale Unterschied zwischen den Güter $1=1, \ldots, n+2$ und den Umweltbelastungsniveaus der ökonomie wird durch die nachstehende Definition erreichbarer Allokationen verdeutlicht.

Definition 2:

Eine Allokation $\left[\left(x^{i}, s^{i}\right),\left(y^{j}\right),(e, z)\right]$ ist in ökonomie $E \equiv\left\{\left[x^{i}, u_{i}, \omega^{i}\right]\right.$, $\left.\left[Y^{j}\right],\left[\Theta_{i j}\right]\right\}$ erreichbar, wenn die nachstehenden Bedingungen erfüllt werden: ${ }^{10)}$

$\left(x^{i}, s^{i}\right) \in x^{i} \quad$ für jedes $i=1, \ldots, N$

(b) $\quad y^{j} \in Y^{j}$ für jedes $j=1, \ldots, K$ und $(e, z) \in Y^{K+1}$

(c.1) $\quad \Sigma \mathrm{x}^{i}+\mathrm{e}=\Sigma \mathrm{y}^{j}+\left(\Sigma \omega^{\mathrm{i}}, 0,0\right)$

(c.2) $s^{i}=z$ für alle $i=1, \ldots, N$

In der Definition 2 kommt durch (c.2) zum Ausdruck, daß Umweltbelastungen in ökonomie E öffentliche "bads" sind. M.a.W. kein Konsument der ökonomie kann sich der vom Umweltproduzenten "bereitgestellten" Umweltbelastung entziehen, d.h. das sogenannte NichtAusschlußprinzip besitzt im Umweltbereich Gültigkeit. Die Güter $1=1, \ldots, n+2$ hingegen sind reine private Güter, womit das Ausschlußprinzip Gültigkeit besitzt. Daher ist eine notwendige Bedingung einer erreichbaren Allokation, die in (c.1) der obigen Definition zum Ausdruck gebrachte Gleichheit zwischen der Summe der individuellen Nachfrage nach diesen Gütern und der summe der individuellen Angebote dieser Güter. Uber die Teile (a) und (b) der Definition wird gefordert, daß bei einer erreichbaren

10) Wird der Summierungsoperator $\Sigma$ nicht spezifiziert, gilt in allen weiteren Ausführungen bei "Summation über $i$ " $i=1, \ldots, N$ und bei "Summation über $j " j=1, \ldots, k$. Somit ist $z \cdot B \cdot \Sigma x^{i}$ $\operatorname{als} \sum_{i=1}^{N} x^{i} z u$ lesen. 
Allokation dej Konsumplan jedes Konsumenten und der Produktionsplan jedes produzenten für diesen erreichbar ist, d.h. in dessen Konsum- bzw. Produktionsmenge liegt. Die Gleichheit zwischen der tatsächlich vorliegenden Umweltbelastung und der vom Umweltproduzenten bereitgestellten Umweltbelastung in einem erreichbaren Zustand wird iiber (b) und (c.1) der vorstehenden Definition impliziert.

Lemma 3:

Sind die Annalumen (a.1), (a.2), (a.3), (a.4), (b), (c.1) erfüllt, ist die Menge der erreichbaren Allokationen der ökonomie $\mathrm{E}$ beschränkt.

Beweis :

Sei $x_{L}^{i} \equiv\left\{x^{i} \mid\left(x^{i}, s^{i}\right) \in x^{i}\right\}$ und $y_{e} \equiv\left\{e \mid(e, z) \in Y^{K+1}\right\}$. Zunächst gilt wegen der Annahme (a.1), (a.2), (b), (c.1), daß $X_{L}^{i}, Y^{j}, Y_{e}$ abgeschlossene, konvexe Mengen sind, die jeweils Null als Element enthalten, wobei $x_{L}^{i}, Y_{e} \subset R_{+}^{n+2}$. Da $\circ \in x_{L}^{i} \subset R_{+}^{n+2}$ folgt $\left[\mathrm{x}^{i} \in \mathrm{X}_{\mathrm{L}}^{i}\right.$ für jedes $i$ und $\Sigma \mathrm{x}^{i} \leqq$ o] impliziert [ $\mathrm{x}^{i}=$ o für alle $\left.i\right]$. $\circ \in Y^{j}$ für jedes $j$ impliziert $\circ \in Y \equiv \sum_{\Sigma}^{K} Y^{j}$, womit wegen Annahme (a.3) $Y \cap R_{+}^{n+2}=\{0\}$ gilt.

Dies garantiejt wegen $\circ \in \Sigma \mathrm{X}_{\mathrm{L}}^{\mathrm{i}} \subset \mathrm{R}_{+}^{\mathrm{n}+2}$ und $\circ \in \mathrm{Y}_{\mathrm{e}} \subset \mathrm{R}_{+}^{\mathrm{n}+2}$ aber $\Sigma \mathrm{X}_{\mathrm{L}}^{\mathrm{i}} \cap\left(\mathrm{Y}-\mathrm{Y}_{\mathrm{e}}\right)=\{\mathrm{O}\}$.

Da $\mathrm{Y} \cap \mathrm{R}_{+}^{\mathrm{n}+2^{\mathrm{e}}}=$ : o\} gilt: $\Sigma \mathrm{Y}^{\mathrm{j}} \geqq 0$ impliziert $\Sigma \mathrm{y}^{\mathrm{j}}=0$. Daher kann wegen $\circ \in Y^{j}, Y \cap(-Y)=\{\circ\}$ über eine $z u$ Debreu (1976), S. 52 analoge Überlegung, die Gültigkeit der Aussage " $\left[y^{j} \in Y^{j}\right.$ für alle $j=1, \ldots, k$ und $\left.\Sigma y^{j} \geqq 0\right]$ impliziert $\left[y^{j}=\right.$ o für alle $\left.j=1, \ldots, k\right] "$ bewiesen werden. Damit zeigt man durch Anwendung derselben Argumente wie Pethig (1979), S. 49 f die Beschränktheit der Menge der erreichbaren Allokationen der ökonomie E.

$$
\text { O.E.D. }
$$

4. Der Allokarionsmechanismus der Modellökonomie

Wie bereits in der zuvor gegebenen Beschreibung der ökonomie E angedeutet, wird aufgrund der zuordnung von Gewinnanteilen und Anfangsausstattungen auf einzelne Konsumenten, von einer ökono- 
mie mit Privateigentum im Sinne Debreus (1976) ausgegangen. Allokationsentscheidungen im privaten Güterbereich werden in der ökonomie über den Markt koordiniert. Da alle privaten Güter marktfähig sind, gibt es für jedes dieser Güter einen einheitlichen Preis, wobei $p_{1} \in R_{+}$den Preis des Gutes $1=1, \ldots, n+2$ und $\left(\mathrm{p}_{\mathrm{x}}, \mathrm{p}_{\mathrm{e}}\right) \in \mathrm{R}_{+}^{\mathrm{n}+2}$ mit $\mathrm{p}_{\mathrm{x}} \equiv\left(\mathrm{p}_{1}, \ldots, \mathrm{p}_{\mathrm{n}}\right), \mathrm{p}_{\mathrm{e}} \equiv\left(\mathrm{p}_{\mathrm{n}+1}, \mathrm{p}_{\mathrm{n}+2}\right)$ das Preissystem privater Güter bezeichnet. Die Besonderheit der Güter $1=n+1, n+2$ liegt darin, daß es aufgrund der Konstruktion der ökonomie genau einen Nachfrager für diese Güter, nämlich den Umweltproduzenten, gibt. Umweltbelastungen oder Immissionen sind wegen des NichtAusschlußprinzips nicht marktfähig. Dennoch werden diesen gesellschaftliche Bewertungskennziffern $p_{n+3}, p_{n+4} \in R_{+}$zugeordnet und $p_{s} \equiv\left(p_{n+3}, p_{n+4}\right)$ als Umweltbelastungspreissystem bezeichnet. Das von den Konsumenten einer Region angestrebte Qualitätsniveau der Umwelt dieser Region wird in der ökonomie über eine Mehrheitsabstimmung ermittelt. Jeder Konsument ist deshalb mit einem "Stimmzettel" ausgestattet, auf welchem er bei der Wahl das von ihm angestrebte Immissionsniveau bzw. seine individuelle Umweltbelastungsnachfrage vermerkt. Ein Wahlleiter ermittelt dann das Wahlergebnis, indem er aus allen ihm mitgeteilten Umweltbelastungsnachfragen die "Medianumweltbelastung" bestimmt. Im folgenden werden zunächst die Entscheidungskalküle eines Konsumenten zur Bestimmung seiner individuellen Umweltbelastungsnachfrage beschrieben. Daran anschließend wird gezeigt, daß die den Präferenzen des Konsumenten über dem Politikraum zuordenbare indirekte Nutzenfunktion streng quasi-konkav ist.

Bei der Bestimmung seiner individuellen Umweltbelastungsnachfrage steht ein Konsument folgendem Entscheidungsproblem gegenüber. Für gegebene Preiseprivater Güter setzt sich das Einkommen eines Konsumenten aus dem Wert seiner Anfangsausstattung und den an ihn fließenden Gewinnzahlungen der $(\mathrm{K}+1)$ produzenten zusammen. Das Einkommen wird dazu verwendet, private Güter und Umweltbelastungen nachzufragen. Dabei geht der Konsument davon aus, daß er pro nachgefragter Umweltbelastungseinheit eine dem Umweltbelastungspreis $p_{s}$ proportionale Entschädigung $\beta_{i} p_{s}$ erhält. De- 
finiert man die Indexfunktion $\mathrm{k}$ als

$$
k(i)=\left\{\begin{array}{l}
1 \text { für } i=N_{1}+1, \ldots, N \\
2 \text { für } i=1, \ldots, N_{1}
\end{array}\right.
$$

besteht das optimierungskalkuil des Konsumenten i darin, bei gegebenem Preissystem $\mathrm{p} \equiv\left(\mathrm{p}_{\mathrm{x}}, \mathrm{p}_{\mathrm{e}}, \mathrm{p}_{\mathrm{s}}\right)$, gegebener Umweltbelastung derjenigen Region, in welcher er nicht lebt $s_{k(i)}^{i}=s_{k}(i)$ und gegebenem Einkommen, diejenige Güterkombination $\left(x^{i}, s^{i}\right)$ seiner Konsummenge auszusuchen, welche unter Beachtung seiner Budgetrestriktion die seinen Präferenzen zugeordnete Nutzenfunktion maximiert. Di.e Projektion dieser "besten" $(n+4)-T u p e l\left(x^{i}, s^{i}\right)$ in ihre $(n+3)$-te oder $(n+4)$-te Koordinate ist dann die individuelle Nachfrage des Konsumenten nach Umweltbelastungen in seiner Region. Dies kann wie folgt formalisiert werden. 11)

$$
\begin{aligned}
& \sigma_{i}\left(p, s_{k, i}\right) \equiv \operatorname{proj}\left[1, h_{i}\left(p, s_{k(i)}\right)\right] \text { mit } 1=n+3, n+4,1 \neq k(i)+n+2 \\
& \text { und } i=1, \ldots, N \text { wobei } h_{i}\left(p, s_{k}(i)\right) \equiv\left\{\left(x^{i}, s^{i}\right) \in B_{i}\left(p, s_{k(i)}\right) \mid\right. \\
& \left.u_{i}\left(x^{i}, s^{i}\right) \geqq u_{i}(x, s) \text { für alle }(x, s) \in B_{i}\left(p, s_{k(i)}\right)\right\}
\end{aligned}
$$

Die Budgetkorrespondenz $\mathrm{B}_{i}$ ist dabei definiert als

$$
\begin{aligned}
& B_{i}\left(p, s_{k(i)}\right) \equiv\left\{\left(x^{i}, s^{i}\right) \in x^{i} \mid s_{k(i)}^{i}=s_{k(i)} \text { und }\left(p_{x}, p_{e}\right) x^{i}-\right. \\
& \left.\beta_{i} p_{s} s^{i} \leqq I_{i}(p)\right\} \text { wobei } I_{i}(p) \equiv p_{x} \omega^{i}+\sum_{j=1}^{K+1} \theta_{i j} \pi_{j}(p) .
\end{aligned}
$$

$\sigma_{i}\left(p, s_{k(i)}\right)$ ist die Projektion der Nachfrage $h_{i}\left(p, s_{k}(i)\right.$ des $i-t e n$ Konsumenten in deren $(n+3)$-te oder $(n+4)$-te Koordinate und gibt damit für gegebenes Preissystem $p$ und gegebene Umweltbelastung $s_{k(i)}$ die "nitzenmaximale" Umweltbelastungsnachfrage des Konsumenten für die ihm zugeordnete Region an. Die Budgetkorrespondenz $B_{i}$ beschreibt die Menge aller Konsummöglichkeiten, die der Budgetrestriktion des $i$ genügen und in dessen Konsummenge $x^{i}$ liegen.

11) Sei $A \subset R^{m}$, so wird im folgenden proj Projektionsfunktion genannt und es gilt proj $[i, A] \equiv\left\{a_{i} \in R \mid a_{i}\right.$ ist die $i-t e$ Komponente eines Elementes $\left.a \in A \subset R^{m} m \frac{1}{i} t \leqq{ }_{m}\right\}$. 
$\sum_{j=1}^{K+1} \Theta_{i j}{ }^{\pi} j(p)$ sind die beim Preissystem p dem Konsumenten zufließenden Gewinnzahlungen und $\beta_{i} p_{s} s^{i}$ kann als die, dem Konsumenten zugeordnete "Umweltbelastungszahlung" interpretiert werden. Der Vektor $\beta_{i} \in \mathrm{R}_{+}^{2}$ ist ein exogen gegebener Verteilungsparameter, wobei $\sum_{i=1}^{\pi} \beta_{i}=(1,1)$ erfüllt ist. Damit ist $\beta_{i} p_{s}$ als "personalisierter Preis" der Umweltbelastungen interpretierbar. Der Gegensatz zu den sogenannten "personalisierten Preisen" im Sinne Lindahls bildet in diesem Modellzusammenhang die Exogenität des vektor $B \equiv\left(\beta_{1}, \ldots, \beta_{N}\right)$.

Nachdem die individuellen Entscheidungskalküle bei einer Abstimmung über das Ausmaß der Bereitstellung an Umweltqualität beschrieben sind, interessiert der spezifische Abstimmungsmodus zur Ermittlung der Umweltqualitätswünsche des Konsumsektors der Ökonomie. Zunächst wird davon ausgegangen, daB ein Konsument nur in derjenigen Region über das Abstimmungsverfahren ein Mitspracherecht bei der Umweltqualitätsfestlegung hat, der er durch die vorgenommene Indizierung fest zugeordnet ist. Damit entfallen die im Abschnitt über die Mehrheitswahl skizzierten Probleme der Bestimmung von Wahlsiegern über mehrdimensionalen Politikräumen. Aus diesem Grunde und wegen der durch (1) gegebenen Definition der individuellen Umweltbelastungsnachfragen wird der Politikraum der Region $1=1,2$ durch $S_{1}=R_{+}$beschrieben. ${ }^{12)}$ wie durch die Beziehungen (1),(2) beschrieben, ermittelt ein Konsument aus Region 1 bei gegebenem Preissystem, gegebenem Einkommen und gegebener Umweltbelastung der Region, in welcher er nicht lebt,

12) Nach Annahme (c) ist $\left(x^{i}, s\right) \in x^{i}$ nur dann erfüllt, wenn $s \in R_{+}^{2}$. Da $\beta_{i p s} \in R_{1}^{2}$ gilt, liegen für jedes $s \in S_{1} \times S_{2}$ semipositive Umweltbelastungszahlungen vor, womit bei $I_{i}(p) \geqq 0$ für jeden Konsumenten ein Überlebenskonsum garantiert ist. Wäre hingegen $\beta_{i} p_{S} \in R$ und $s$ eine durch Abstimmung festgelegte Umweltbelastung, könnte à priori $I_{i}(p)+\beta_{i} p_{S} s<0$ für einige Konsumenten nicht ausgeschlossen werden. In diesem Falle müßte zur Vermeidung des Bankrotts einzelner Konsumenten der Politikraum analog zu Slutzky (1977), S.308 definiert werden. 
über den Politikraum $S_{1}$ seine individuelle Umweltbelastungsnachfrage, um sie einem Wahlleiter mitzuteilen. Offensichtlich können daher bei g.egebenem preissystem $p \in R_{+}^{n+4}$ und gegebenem Einkommen $I_{i}(p)>0$ die Präferenzen eines Konsumenten über der Menge $\mathrm{S} \equiv \mathrm{S}_{1} \times \mathrm{S}_{2}$ durch eine indirekte Nutzenfunktion $\mathrm{v}_{i}: \mathrm{S} \rightarrow \mathrm{R}$ abgebildet werden. ${ }^{13)}$ Die durch die Funktion $v_{i}$ abgebildeten präferenzen erhält man daher über die Lösung eines Maximierungsproblems. Dabei ist $v_{i}$ für $i=1, \ldots, N$ definiert als

$$
v_{i}(s)=\max \left\{u_{i}(x, s) \mid x \in B_{i}^{b}(p, s)\right\}
$$

wobei $B_{i}^{b}(p, s) \equiv\left\{x \in x_{L}^{i} \mid\left(p_{x}, p_{e}\right) x \leqq I_{i}(p)+\beta_{i} p_{s} s\right\}$ und $x_{L}^{i} \equiv$ $\left\{x \in R_{+}^{n+2} \mid(x, s) \in x^{i}\right\}$ gilt. $\mathrm{B}_{i}^{\mathrm{b}}(\mathrm{p}, \mathrm{s})$ wird die bedingte Budgetmenge des Konsumenten $i$ genannt und gibt für gegebenes Preissystem und gegebene Umweltbelastungsnachfrage die Menge erreichbarer Konsummöglichkeiten privater Güter des $i$ an. Bezeichnet $H_{i}$, definiert als $H_{i}(p, s)=$ $\left\{x \in B_{i}^{b}(p, s) \mid u_{i}(x, s)=v_{i}(s)\right\}$ die bedingte Nachfragefunktion des Konsumenten $i$ nach privaten Gütern und wird für festes $p \in R_{+}^{n+4}$ geschrieben $H_{i}(p, s)=\tilde{H}_{i}(s)$, gilt offensichtlich

$$
v_{i}(s)=u_{i}\left(\widetilde{H}_{i}(s), s\right) \quad \text { mit } \tilde{H}_{i}(s)=H_{i}(p, s) \text { und p konstant. }
$$

Für den Fall eines privaten Gutes und eines Umweltbelastungsindikators $s \in R_{+}$:äßt sich die Konstruktion der indirekten Nutzenfunktion $v_{i}$ durch das nachstehende Schaubild 4 verdeutlichen. Im ersten Quadranten dieses Schaubilds ist die indirekte Nutzenfunktion einess Konsumenten i für gegebene Preise und gegebenes Einkommen $I_{i}(p)$ eingezeichnet. Diese indirekte Nutzenfunktion wird konstruiert, indem die im II. Quadranten des Schaubildes 4 vorliegenden Wertes der Schnitt- und Tangentialpunkte der Indifferenzkurven des Konsumenten $i$ mit der Budgetgeraden dieses Konsumenten in Quadrant I übertragen werden. Schaubild 4 legt die

13) Der Ausdruck "indirekte" Nutzenfunktion ist hier nicht im Sinne Debreus (1976) als Abhängigkeit des Nutzenindexes von Preisen und Vermögen gebraucht, sondern gibt für gegebene Preise, gegebenes Vermögen, gegebenes $s_{k}(i)$ den Nutzenindex alternativer umweltbelastungsniveaus an. 


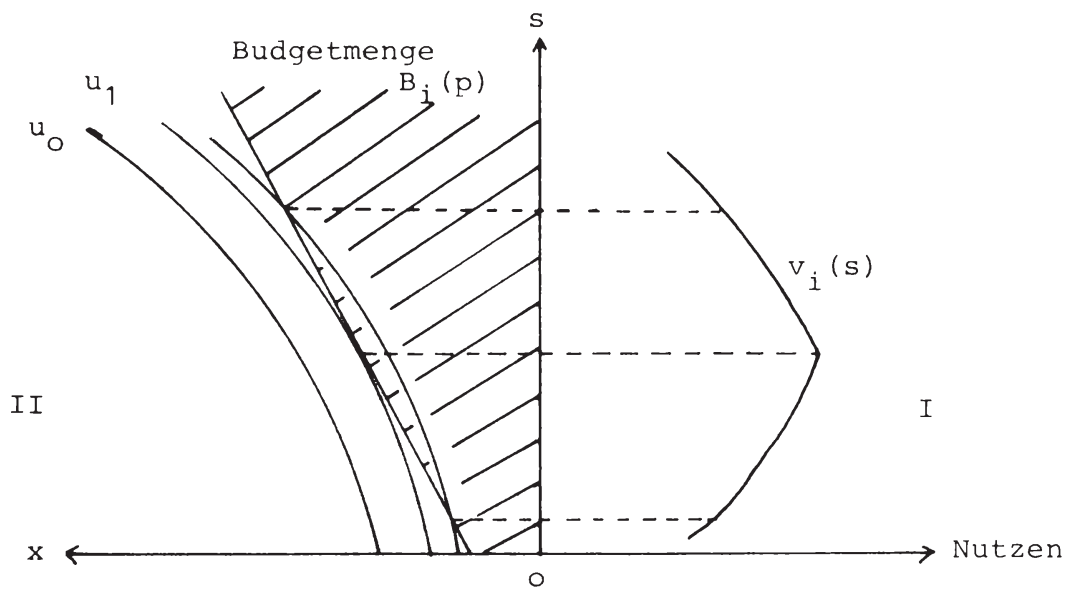

Schaubild 4

Vermutung nahe, daß stetigkeit und strenge Quasi-Konkavität der Nutzenfunktion $u_{i}$ die strenge Quasi-Konkavität der indirekten Nutzenfunktion $\mathrm{v}_{i}$ impliziert. Diese Vermutung wird durch den nachstehenden Hilfssatz präzisiert und bewiesen.

Lemma $4:$

Seien die Annahmen (c.1), (c.2) erfüllt, die Konsummengen abgeschlossen und beschränkt und $I_{i}(p)>0$. Unter diesen Voraussetzungen ist die indirekte Nutzenfunktion jedes Konsumenten bei gegebenen semi-positiven Preisen streng quasi-konkav.

Beweis:

Sei $\bar{x}_{L}^{i} \equiv\left\{x \mid(x, s) \in \bar{x}^{i}\right\}$ und $\bar{s}_{i} \equiv\left\{s \mid(x, s) \in \bar{x}^{i}\right\}$ und $\bar{x}^{i} \subset x^{i}$ konvex und kompakt. Da im folgenden $p$ und $i$ fixiert ist, wird der Index $i$ und die Argumentvariable $p$ vernachlässigt. Zunächst zeigt man uiber Standardargumente die Stetigkeit der Budgetkorrespondenz $B^{b}: \bar{S} \rightarrow \bar{X}_{L}$ definiert als $B^{b}(s)=\left\{x \in \bar{x}_{L} \mid\left(p_{x}, p_{e}\right) x \leq I+\right.$ $\beta \mathrm{p}_{\mathrm{s}} \mathrm{s}^{\mathrm{s}} \cdot \mathrm{Da} \overline{\mathrm{x}}_{\mathrm{L}}$ kompakt, $\mathrm{B}^{\mathrm{b}}$ stetig und die Nutzenfunktion u stetig ist, folgt nach dem Satz von Berge die stetigkeit der indirekten Nutzenfunktion $\mathrm{v}: \overline{\mathrm{s}} \rightarrow \mathrm{R}$, definiert als $\mathrm{v}(\mathrm{s})=\max \{\mathrm{u}(\mathrm{x}, \mathrm{s}) \mid \mathrm{x} \in \mathrm{B}(\mathrm{s})\}$ 
und die Oberhalb-Semistetigkeit der Abbildung $\widetilde{\mathrm{H}}: \overline{\mathrm{S}} \rightarrow \overline{\mathrm{X}}_{\mathrm{L}}$ ciefiniert als $\widetilde{H}(s)=\left\{x \in B^{b}(s) \mid u(x, s)=v(s)\right\}$. Da u streng quasi-konkav ist, enthält $\widetilde{\mathrm{H}}(\mathrm{s})$ genau ein Element, womit aufgrund der Oberhalb-Semistetigkeit $\tilde{\mathrm{H}}$ stetig ist.

Strenge Quasi-Konkavität der indirekten Nutzenfunktion v verlangt, daß für beliebige $\overline{\mathrm{s}}, \overline{\overline{\mathrm{s}}} \in \overline{\mathrm{s}} \operatorname{mit} \overline{\mathrm{s}} \neq \overline{\overline{\mathrm{s}}}, \mathrm{v}(\overline{\mathrm{s}}) \geqq \mathrm{v}(\overline{\bar{s}}), \overline{\mathrm{x}}=\widetilde{\mathrm{H}}(\overline{\mathrm{s}})$, $\overline{\overline{\mathrm{x}}}=\widetilde{\mathrm{H}}(\overline{\overline{\mathrm{s}}})$ die Bedingung $\mathrm{v}\left(\mathrm{s}^{\lambda}\right)>\mathrm{v}(\overline{\overline{\mathrm{s}}})$ für $0<\lambda<1$ und $\mathrm{s}^{\lambda} \equiv \lambda \overline{\mathrm{s}}+(1-\lambda) \overline{\overline{\mathrm{s}}}$ erfüllt ist. Die Konvexität der Budgetmenge $B(p) \equiv\{(x, s) \in \bar{X}$ $\left.\left(p_{x}, p_{e}\right) x-\beta p_{s} s \stackrel{I}{=}(p)\right\}$ und $(\widetilde{H}(\bar{s}), \bar{s}),(\widetilde{H}(\overline{\bar{s}}), \overline{\bar{s}}) \in B(p)$ impliziert $\left(\lambda \tilde{\mathrm{H}}(\overline{\mathrm{s}})+(1-\lambda) \widetilde{\mathrm{H}}(\overline{\overline{\mathrm{s}}}), \mathrm{s}^{\lambda}\right) \in \mathrm{B}(\mathrm{p})$, und per Definition gilt $\left(\lambda \widetilde{\mathrm{H}}(\overline{\mathrm{s}})+(1-\lambda) \widetilde{\mathrm{H}}(\overline{\bar{s}}), \mathrm{s}^{\lambda}\right) \in \mathrm{B}^{\mathrm{b}}\left(\mathrm{s}^{\lambda}\right)$, womit $\mathrm{u}\left(\lambda \widetilde{\mathrm{H}}(\overline{\mathrm{s}})+(1-\lambda) \widetilde{\mathrm{H}}(\overline{\bar{s}}), \mathrm{s}^{\lambda}\right)$ ein erreichbarer Nutzenindex ist. Da $v\left(s^{\lambda}\right)=\max \left\{u\left(x, s^{\lambda}\right) \mid x \in B^{b}\left(s^{\lambda}\right)\right\}$ $=u\left(\widetilde{H}\left(s^{\lambda}\right), s^{\lambda}\right)$ ist aber auch $u\left(\widetilde{H}\left(s^{\lambda}\right), s^{\lambda}\right) \geqq u(\lambda \widetilde{H}(\bar{s})+(1-\lambda)$ $\tilde{\mathrm{H}}(\overline{\overline{\mathrm{S}}}), \mathrm{s} \lambda)$ erfüllt, was wegen der strengen Quasi-Konkavität der. Nutzenfunktion $u\left(\lambda \widetilde{\mathrm{H}}(\overline{\mathrm{s}})+(1-\lambda) \widetilde{\mathrm{H}}(\overline{\overline{\mathrm{s}}}), \mathrm{s}^{\lambda}\right)>\mathrm{u}(\widetilde{\mathrm{H}}(\overline{\overline{\mathrm{s}}}), \overline{\overline{\mathbf{s}}})=\mathrm{v}(\overline{\overline{\mathrm{s}}})$ impliziert.

$$
\text { Q.E.D. }
$$

Da die Funktion $\mathrm{v}_{i}$ streng quasi-konkav über der Menge $\mathrm{S}=\mathrm{S}_{1} \times \mathrm{S}_{2}$ ist, ist $v_{i}$ auch streng quasi-konkav über der Menge $R_{+} \times\left\{s_{k(i)}\right\} \subset S$. Daher existiert für gegebenes $p \in R_{+}^{n+4}$ und gegebenes $s_{k(i)}^{i}=s_{k(i)}$ nach dem im A.bschnitt über die Mehrheitswahl Gesagten ein Mehrheitswahlgleichgewicht. Weiterhin sind die Voraussetzungen des Lemma 2 erfüllt und das Mehrheitswahlgleichgewicht kann über das Medianmaß errechnet werden. Die Abstimmung zur Ermittlung der regionalen Umweltbelastungsnachfragen wird deshalb derart durchgeführt, daß jeder Konsument bei gegebenen Preisen und gegebenem $s_{k(i)}^{i}=s_{k(i)}$ einem wahlleiter anonym seine individuelle Umweltbelastungsnachfrage mitteilt. Der Wahlleiter bestimmt aus der Menge der mitgeteilten individuellen Umweltbelastungsnachfragen durch Medianbildung über dieser Menge die jeweiligen regionalen Umweltbelastungsnachfragen, womit für die Umweltbelastungsnachfrage der ökonomie gilt:

$$
\zeta(p, s)=\operatorname{median}_{i=1, \ldots, N_{1}} \sigma_{i}\left(p, s_{k(1)}\right) \times \operatorname{median}_{i=N_{1}+1, \ldots, N^{1}}\left(p, s_{k(1)}\right)
$$


Wie aus (3) erkennbar, wird davon ausgegangen, daß kein Wahlberechtigter die Möglichkeit der "Stimmenthaltung" nutzt ${ }^{14)}$. Die folgenden Uberlegungen sollen zeigen, daß "Stimmenthaltung" für jeden wähler nicht individuell rational ist. Individuelle Rationalität ist dabei nicht im Hurwicz'schen Sinne gebraucht, wonach die mangelnde Vorteilhaftigkeit der Teilnahme an dem Spiel über den gesamten Prozeßverlauf ausgeschlossen wird, sondern individuelle Rationalität stellt hier auf die Vorteilhaftigkeit der Teilnahme am Spiel auf einer Stufe des Anpassungsprozesses ab. 15) Setzt man voraus, daß die Abstimmungen keinen "Ressourcenverzehr" verursachen, also die "Wahlkosten" für den Einzelnen und die gesamte ökonomie - etwa die Bestimmung der individuellen Umweltbelastungsnachfragen oder die Ermittlung der Medianwerte - gleich null sind, ist es nach dem eben vereinbarten Begriffsinhalt individuell rational, an der Abstimmung teilzunehmen. Denn durch die Teilnahme an der Abstimmung besitzt ein Konsument die Möglichkeit, die "Mediannachfrage" seiner Region $\mathrm{zu}$ seinen Gunsten $\mathrm{zu}$ beeinflussen. Damit gibt es keine Konstellation individueller Umweltbelastungsnachfragen, welche die Position eines Wahlberechtigten bei stimmenthaltung verbessern. Da andererseits Konstellationen der individuellen Umweltbelastungsnachfragen existieren, welche die Verschlechterung der position eines Wahlberechtigten bei stimmenthaltung implizieren, folgt aus dem "Nutzenoptimierungskalkül" jedes Konsumencen dessen Wahlbeteiligung. Das Argument, einzelne Konsumenten zeigen Stimmenthaltung, da ihrer Meinung nach das Umweltproblem der ökonomie in einem nicht näher präzisierten Sinne "aufgebauscht" ist, trifft damit auf die hier beschriebene ökonomie nicht zu.

14) Bei temporärer Stimmenthaltung einzelner Wahlberechtigter kann die später zu zeigende Oberhalb-Semistetigkeit der regionalen Umweltbelastungsnachfragekorrespondenzen nicht behauptet werden. Die Oberhalb-Semistetigkeit der eben genannten Korrespondenzen wird für ein Fixpunktargument beim Existenzbeweis des noch zu definierenden kombinierten Marktund Abstimmungsgleichgewichts benötigt.

15) Die fehlende individuelle Rationalität einer stimmenthaltung gemäß dem Hurwicz'schen Kriterium kann für den Fall eines Umweltindikators und eines Konsumgutes analog zu Dudenhöffer (1980) gezeigt werden. 
Die Entscheidungskalküle der Produzenten der ökonomie E lassen sich durch die folgenden Ausführungen charakterisieren. Die Produzenten $j=1, \ldots, K$ stehen bei gegebenem Preissystem $p \in R_{+}^{n+4}$ dem Problem gegenüber, die gewinnmaximalen Produktionspläne aus ihren Produktionsmengen $\mathrm{Y}^{j}$ auszuwählen. Daher lassen sich die Angebotskorrespondenzen der $j=1, \ldots, K$ Produzenten definieren als

$$
\begin{aligned}
& g_{j}(p)=\left\{y^{j} \in Y^{j} \mid \sum_{l=1}^{n} p_{1} y_{1}^{j}-\sum_{l=n+1}^{n+2} p_{1} y_{1}^{j} \geqq \sum_{l=1}^{n} p_{1} y_{1}-\right. \\
& \left.\sum_{l=n+1}^{n+2} p_{1} y_{1} \quad \text { für alle } y \in Y^{j}\right\} \quad \text { für } j=1, \ldots, k
\end{aligned}
$$

Die Projektion der Menge $g_{j}(p)$ in ihre $(n+1)$ und $(n+2)$-te Koordinate ist das beim Preissystem $p$ vorliegende Emissionsangebot des Produzenten $j$. Der Ausdruck $\sum_{l=n+1}^{n+2} p_{1} y j$ wird die Emissionssteuerzahlung des $j$ genannt und $p_{e} \equiv\left(p_{n+1}, p_{n+2}\right)$ heißt Emissionssteuervektor. Durch Aggregation über alle $j=1, \ldots, k$ erhält man die gesamtwirtschaftliche Angebotskorrespondenz g für ökonomie E als

$$
g(p)=\Sigma g_{j}(p)
$$

Analog zu der einzelwirtschaftlichen Angebotskorrespondenzen bezeichnet hier proj $[n+1, y] \times \operatorname{proj}[n+2, y]$ mit $y \in g(p)$ ein gesamtwirtschaftliches Emissionsangebot beim Preissystem $p$.

Neben den Konsumenten und $\mathrm{K}$ Produzenten ist in ökonomie $\mathrm{E}$ eine sogenannte Umweltbehörde etabliert. Nach dem Sprachgebrauch Pethig's (1979), S.71 besteht diese Behörde lediglich aus einer "technischen" Abteilung, womit die Funktion der Behörde auf die eines Umweltproduzenten reduziert ist. Die Aufgabe der Umweltbehörde oder des Umweltproduzenten besteht deshalb darin, bei gegebenem Preissystem $\mathrm{p} \in \mathrm{R}_{+}^{\mathrm{n}+4}$ unter Beachtung der Umwelttechnologie diejenigen Umweltbelastungen $z \equiv\left(z_{1}, z_{2}\right)$ anzubieten und diejenigen Emissionsmengen nachzufragen, welche die Gewinne der Be- 
hörde aus der "Umweltproduktion" maximieren. 16) Dabei ist vorausgesetzt, daß der Umweltproduzent die in Annahme (b) beschriebene Umwelttechnologie kennt. Daher läßt sich die Umweltbelastungsangebots- und Emissionsnachfragekorrespondenz des Produzenten $\mathrm{K}+1$ formulieren als

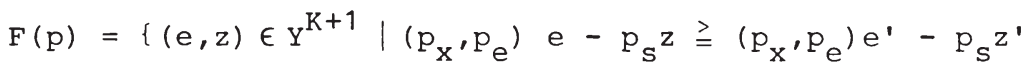

$$
\begin{aligned}
& \text { für alle } \left.\left(e^{\prime}, z^{\prime}\right) \in Y^{K+1}\right\}
\end{aligned}
$$

Die Erlöse des Umweltproduzenten setzen sich wegen $(e, z) \notin Y^{K+1}$ für $e_{l} \neq 0$ mit $l=1, \ldots, n$ aus dem Emissionssteueraufkommen $p_{e}\left(e_{n+1}\right.$ ' $e_{n+2}$ ) zusammen und seine Kosten ergeben sich als Summe der regionalen Umweltbelastungszahlungen $\mathrm{p}_{\mathrm{s}} \mathrm{z}$, wobei wegen o $\in \mathrm{Y}^{\mathrm{K}+1}$ auch $\pi_{\mathrm{K}+1} \geqq 0 \mathrm{gilt}$.

Damit sind für ökonomie E die Kalküle zur Ermittlung der Umweltbelastungsangebote und -nachfragen sowie der Emissionsangebote und -nachfragen vollständig beschrieben. Obwohl im Umweltbereich der ökonomie das Ausschlußprinzip in seiner strengen Form Gültigkeit besitzt, ist es aufgrund des "eingebetteten"

16) Eine andere Variante des "Verhaltens" der Behörde ist die Forderung nach ausgeglichenem Budget der Behörde. Unter dieser Variante ergäben sich die Umweltbelastungsangebote und Emissionsnachfragen der Behörde als die Menge der Tupel $(e, z)$ welche bei gegebenem Preissystem p die Emissionssteueraufkommen und Umweltbelastungszahlungen der Behörde zu null summieren. Der Vorteil einer derart konstruierten Behörde ist darin zu sehen, daß die Konsumentennachfragen nach Umweltbelastungen nicht dadurch "verzerrt" werden, daß "Umweltproduktionsgewinnzahlungen $\Theta_{i K+1} \pi K+1$ dem Einkommen der Konsumenten zugeführt werden. Der Nachteil der genannten Variante besteht darin, daß die Bedingungen erster ordnung für ein Gewinnmaximum $p_{n+3}\left(\partial z_{1} / \partial e_{n+1}\right)-p_{n+1}=0$ und $p_{n+3}\left(\partial z_{1} / \partial e_{n+2}\right)+p_{n+4}$ $\left(\partial \mathrm{z}_{2} / \partial \mathrm{e}_{n+2}\right)-\mathrm{p}_{\mathrm{n}+2}=0$ nicht erfüllt werden, womit die bewertete Utmweltbeflastung einer zusätzlichen Emissionseinheit nicht dem Emissionssteuersatz für diese Einheit entspricht. Ausgeglichenes Budget wie oben beschrieben impliziert daher paretoinferiore Zustände der ökonomie. 
Mehrheitswahlmechanismus möglich, durch Übertragen des traditionellen Angebots- und Nachfrageschemas, einen "Umweltmarkt" zu simulieren. Akteure auf dem Umweltmarkt sind dabei die $j=1, \ldots, k+1$ Produzenten und der "Mediankonsument". Die Zusammenhänge auf dem Umweltmarkt können anhand der nachstehenden Darstellung interpretiert werden. Schaubild 5 vermittelt eine partialanalytische Darstellung des umweltökonomischen Allokationsaspektes der ökonomie E. Für gegebenes Preissystem privater Güter sind den Konsumenten der ökonomie bei den Bewertungskennziffern $\mathrm{p}_{\mathrm{S}}$ individuelle Umweltbelastungsnachfragen $\mathrm{s}^{1}, \ldots, \mathrm{s}^{\mathrm{N}}$ zugeordnet. Gemäß dem beschriebenen Abstimmungsverfahren werden hieraus die regionalen Umweltbelastungsnachfragen $s_{1}$ und $s_{2}$ ermittelt. Diesen Umweltbelastungsnachfragen stehen beim preissystem $p \in R_{+}^{n+4}$ die Umweltbelastungsangebote $z \equiv\left(z_{1}, z_{2}\right)$ gegenüber. Die Koordination der Umweltbelastungsangebote und -nachfragen erfolgt dann durch die Variation der Umweltbewertungskennziffern $\mathrm{p}_{\mathbf{S}}$. Der Anpassungsprozeß ist als Tâtonnement interpretierbar, d.h. überschreitet (unterschreitet) die jeweilige Umweltbelastungsnachfrage bei gegebenen preisen $p$ das jeweilige Angebot, senkt (erhöht) ein Auktionator die Preis $p_{s}$ bis ein Ausgleich beider Größen erreicht ist. Weichen bei diesen "neu" festgesetzten Preisen und weiterhin konstant gebliebenen Emissionssteuersätzen $\mathrm{p}_{e}$ die jeweiligen Emissionsangebote von den Nachfragen nach Emissionen der Behörde $a b$, greift erneut ein Auktionator ein und ändert bei gegebenen Preisen der übrigen Gütern die Steuersätze $\mathrm{p}_{e}$ so lange, bis ein "Emissionsmarkträumungspreis" vorliegt. Bei diesen "neuen" Emissionssteuern überdenkt der Umweltproduzent seine Umweltbelastungsangebote und der Median-Konsument jeder Region seine Umweltbelastungsnachfrage. Ergibt sich dabei, daß die regionalen Umweltbelastungsnachfragen den jeweiligen Angeboten entsprechen, ist der Proze $\beta$ in einem (partiellen) Gleichgewichtszustand angekommen. Liegt keine Utbereinstimmung vor, setzt sich der Prozeß wie oben beschrieben fort.

Um das vorgestellte Modell zu vervollständigen, ist es notwendig, die Entscheidungskalküle der Konsumenten bei ihrer Nachfra- 


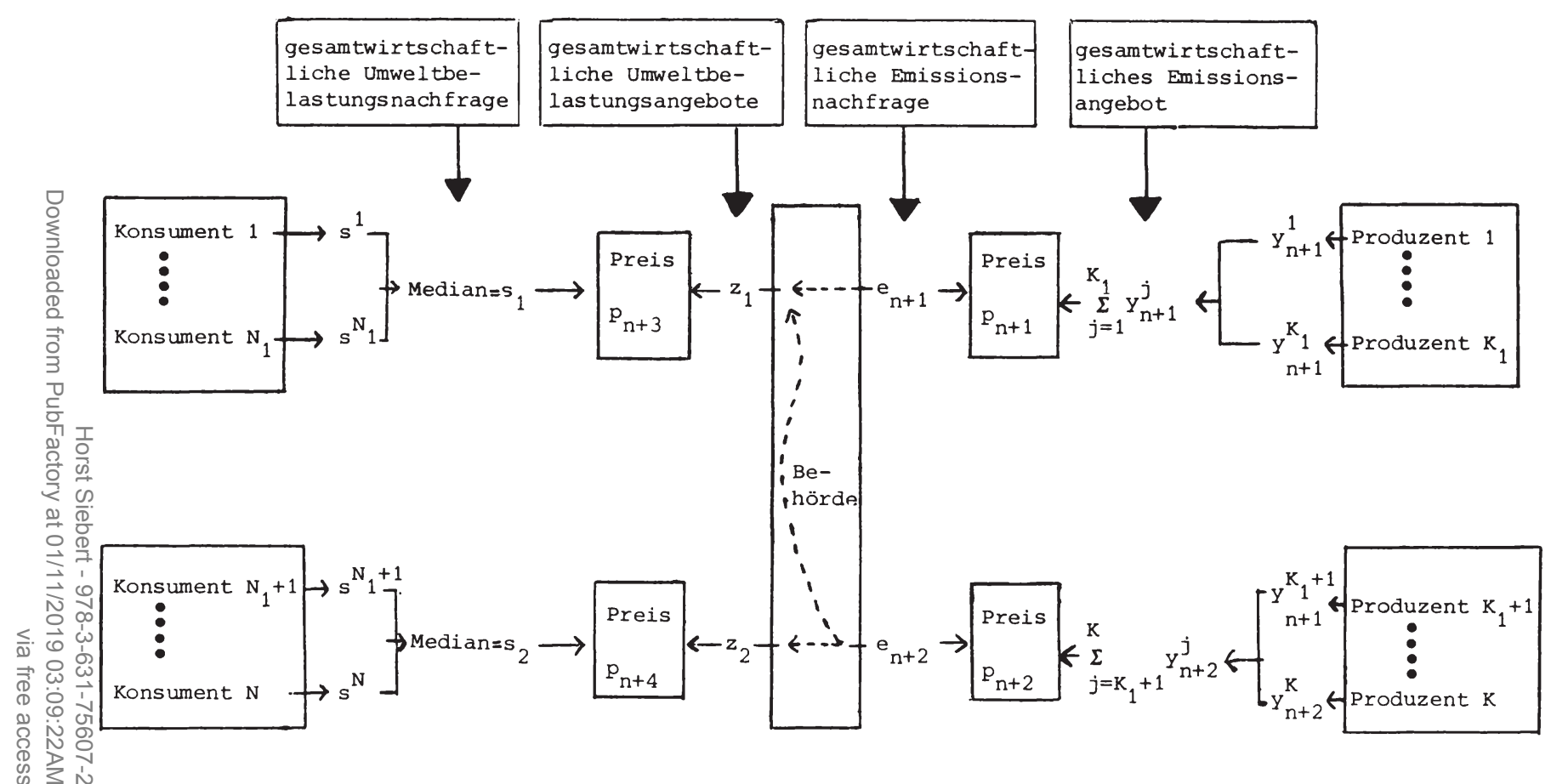

Schaubild 4 
ge und privaten Gütern offenzulegen. Für gegebene gesamtwirtschaftliche Um'weltbelastungsnachfrage s und gegebenes Preissystem $p \in R_{+}^{n+4}$, setzt sich das Einkommen des Konsumenten $i$ aus dem Wert seiner Anfangsausstattung, den an ihn fließenden Gewinnzahlungen der $\mathrm{K}+1$ Produzenten und der ihm zustehenden Umweltbelastungszahlung $B^{i} p_{s} s$ zusammen. Bei gegebenem Einkommen bestimmt dann ein Konsument unter Beachtung der für gegebene gesamtwirtschaftliche Umweltbelastungsnachfrage gültigen Budgetrestriktionen seinen nutzenmaximalen Konsum privater Güter. Dies wird formalisiert durch

$$
\begin{aligned}
& \mathrm{H}_{i}(p, s)=\left\{x^{i} \in B_{i}^{b}(p, s) \mid u_{i}\left(x^{i}, s\right) \geqq u_{i}(x, s)\right. \\
& \text { für alle } \left.x \in B_{i}^{b}(p, s)\right\}
\end{aligned}
$$

wobei die "bedingte" Budgetmenge $\mathrm{B}_{i}^{\mathrm{b}}(\mathrm{p}, \mathrm{s})$ definiert ist als

$$
\begin{aligned}
& B_{i}^{b}(p, s)=\left\{x \in x_{L}^{i} \mid\left(p_{x}, p_{e}\right) x \leqq I_{i}(p)+B_{i} p_{s} s\right\} \text { und } \\
& x_{L}^{i} \equiv\left\{x \in R_{+}^{n+2} \mid(x, s) \in x^{i}\right\}
\end{aligned}
$$

Damit ist eine Zweistufigkeit des Konsumentenkalkül im Modell unterstellt. Auf stufe 1 stehen die einzelnen Konsumenten dem durch die Beziehungen (1), (2) charakterisierten Problem der Bestimmung individueller Umweltbelastungsnachfragen gegenüber. Nachdem jeder Konsument diese optimierungsaufgabe gelöst hat und über die beschriebene Abstimmung die Mediannachfrage nach Umweltbelastungen errechnet ist, stellt diese Umweltbelastungsnachfrage ein Datum für die einzelnen Konsumenten dar. Daher sieht sich jeder Konsument - mit Ausnahme des Medianwähler geänderten einzelwirtschaftlichen Bedingungen gegenüber, womit sich das in den Beziehungen (7), (7a) geschilderte Maximierungsproblem der Stufe 2 stellt.

Damit ist der Allokationsmechanismus der ökonomie E hinreichend beschrieben und es interessieren in diesem Zusammenhang Bedingungen, welche die Existenz eines kombinierten Markt- und 
Abstimmungsgleichgewichts der ökonomie garantieren. ${ }^{17)}$ was unter einem kombinierten Markt- und Abstimmungsgleichgewicht verstanden wird, ist in der nachstehenden Definition gesagt.

Definition 3 :

Ein Markt- und Abstimmungsgleichgewicht der Ökonomie $E$ ist ein $(\mathrm{N}+\mathrm{K}+1+1)-\mathrm{Tupel}\left[\left(\hat{\mathrm{x}}^{\mathrm{i}}, \hat{\mathrm{s}}\right),\left(\hat{\mathrm{Y}}^{j}\right),(\hat{\mathrm{e}}, \hat{\mathrm{z}}), \hat{\mathrm{p}}\right]$ mit $\hat{\mathrm{p}} \geq 0 \operatorname{derart}, \mathrm{da} \beta$

(a) $\quad \hat{x}^{i} \in H_{i}(\hat{p}, \hat{s})$ für jedes $i=1, \ldots, N$

(b) $\quad \hat{y}^{j} \in g_{j}(\hat{p}) \quad f u ̈ r$ jedes $j=1, \ldots, K$ und $(\hat{e}, \hat{z}) \in F(\hat{p})$

(c.1) $\quad \Sigma \hat{\mathrm{x}}^{i}+\hat{\mathrm{e}}=\Sigma \hat{\mathrm{Y}}^{j}+\left(\Sigma(1,)^{i}, 0,0\right)$

(c.2) $\quad \hat{\mathrm{s}}=\hat{\mathrm{z}}$

(d) $\quad \hat{s} \in \zeta(\hat{p}, \hat{s})$

Die Bedingung (b) der Definition 3 besagt, daß der realisierte Produktionsplan jedes Produzenten - einschließlich des Umweltproduzenten - bei Gleichgewichtspreissystem $\hat{p}$ ein "gewinnmaximaler" Produktionsplan ist. Bedingung (a) fordert, daß beim Preissvstem $\hat{p}$ und beim Umweltbelastungsniveau $\hat{\mathbf{s}}$ jeder Konsument die für ihn nutzenmaximale Menge privater Güter $\hat{x}^{i}$ nachfragt. Das nutzenmaximale Umweltbelastungsniveau eines Konsumenten, also $\sigma_{i}$ ( $\hat{p}$, $\hat{s}_{k(i)}$, muß dabei nicht mit dem Umweltbelastungsniveau $\hat{s}$ übereinstimmen. Uber die Bedingung (d) der Definition 3 wird gefordert, daß im Gleichgewicht die tatsächlich vorliegenden Umweltbelastungen der beiden Regionen den "Mediannachfragen" nach Umweltbelastungen entsprechen. Oder m.a.W., daß die beim preissystem $\hat{p}$ über Mehrheitswahlen in den jeweiligen Regionen ermittelten Umweltbelastungen mit den tatsächlich vorliegenden Umweltbelastungen übereinstimmen. Bei der Interpretation der Bedingung (c.1), (c.2) kann analog zur Interpretation von (c.1) der Definition 2 argumentiert werden.

17) Slutzky (1977), S. 309, nennt ein derartiges Gleichgewicht "General Bolitical Equilibrium". 
Satz (Existenz):

Unter den Annahmen (a), (b), (c) sowie der Annahme (c') $\quad B_{i}^{1} \neq 0$ für $i=1, \ldots, N_{1}$ und $\beta_{i}^{2} \neq 0$ für $i=N_{1}+1, \ldots, N$ mit $\beta_{i} \equiv\left(\beta_{i}^{1}, \beta_{i}^{2}\right)$

existiert ein Markt- und Abstimmungsgleichgewicht für ökonomie E.

Anhang:

Beweis des Satzes über die Existenz eines Marktund Abstimmungsgleichgewichtes

1. Teil: Kompaktifizierung der ökonomie

Zunächst gilt, daß die Menge der erreichbaren Zustände der ökonomie E nach Hilfssatz 3 beschränkt ist. Damit ist die Menge der erreichbaren Konsumpläne (Produktionspläne) jedes Individuums in ökonomie E', also die für ihn erreichbare Konsum- bzw. Produktionsmenge beschränkt. Sei nun L ein abgeschlossener würfel im $\mathrm{R}^{\mathrm{n}+4}$ mit Mittelpunkt null, der diese $\mathrm{N}+\mathrm{K}+1$ Mengen in seinem Inneren enthëlt. Es wird für $i=1, \ldots, N$ und $j=1, \ldots, k$ definiert

$$
\hat{X}^{i}=X^{i} \cap I, \hat{Y}^{j} \times 0 \times 0=\left[Y^{j} \times 0 \times \cdot j \cap L \text {, und } \hat{Y}^{K+1}=Y^{K+1} \cap L\right.
$$

Zunächst gilt: für $j=1, \ldots, K$, daß $\hat{\mathrm{Y}}^{j}$ als schnittmenge abgeschlossener, konvexer Mengen, die null als Element enthalten ebenfalls diese Eigenschaften aufweist. Da L beschränkt ist, ist auch $\hat{\mathrm{Y}}^{j}$ beschränkt. Analog ergibt sich die Kompaktheit und Konvexität der $\hat{\mathrm{X}}^{\mathrm{i}}$ und die Kompaktheit von $\hat{\mathrm{Y}}^{\mathrm{K}+1}$.

2. Teil: Eigenschaften der Angebots - und Nachfragekorrespondenzen

Sei $c_{i} \equiv\left\{(p, s) \in R_{+}^{n+6}\right.$ es gibt ein $x^{i} \in B_{i}^{b}(p, s)$ derart, daß 
$u_{i}\left(x^{i}, s\right) \geqq u_{i}(x, s)$ für alle $\left.x \in B_{i}^{b}(p, s)\right\}$. Da die Konsumenten nach Annahme (c.2) in bezug auf die Güter $1=1, \ldots, n$ unersättlich sind, folgt $(p, s) \notin c_{i}$ für $p \equiv\left(p_{x}, p_{e}, p_{s}\right)$ und $p_{x}=0$, womit die Menge $\mathrm{H}_{i}(\mathrm{p}, \mathrm{s})$ für den genannten Punkt nicht definiert ist. Da die Korrespondenzen $\zeta, g, F, H$ homogen vom Grade null in den Preisen sind, $p \in R_{+}^{n+4}$ gilt und $(p, s) \notin C_{i}$ für $p_{x}=0$ gilt, kann ohne Beschränkung der Allgemeinheit angenommen werden, daß das Preissystem in $P \equiv\left\{p \in R_{+}^{n+4} \mid \sum_{l=1}^{n+4} p_{1}=1\right\}$ liegt.

Im folgenden werden die ursprünglich unbeschränkten Mengen durch ihre Kompakte, durch den Bogen " $\wedge$ " gekennzeichneten Teilmengen ersetzt. Auf diese Weise erhält man die kompakte ökonomie $\hat{E}$. Bezüglich der Eigenschaften der Gewinnfunktion $\hat{\pi}_{j}$ definiert als $\hat{\pi}_{j}(p)=\max \left\{\sum_{1=1}^{n} \underline{p}_{1} y_{1}-{ }_{1=n+1}^{n+2} p_{1} y_{1} \mid y \in \hat{Y}^{j}\right\}$ für $j=1, \ldots, k$ und $\hat{\pi}_{K+1}(p)=\max \left\{\left(p_{x}, p_{e}\right) e-p_{S} z \mid(e, z) \in \hat{Y}^{K+1}\right\}$ und der Angebotskorrespondenzen $\hat{g}, \hat{F}$ erhält man unmittelbar

Lemma 5:

Die Gewinnfunktionen $\hat{\pi}_{j}$ sind stetig auf $P$ für alle $j=1, \ldots, K+1$ mit $\hat{\pi}_{j}(p) \geqq 0$ und die Angebotskorrespondenzen $\hat{g}$ und $\hat{F}$ sind oberhalb-semistetig auf $\mathrm{P}$ mit jeweils nichtleeren konvexen Bildmengen $\hat{g}(p), \hat{F}(p)$.

Da jedes $\widehat{Y}^{j}$ kompakt ist und $\left[\sum_{l=1}^{n} p_{1} y_{1}^{j}-\sum_{l=n+1}^{n+2} p_{1} y_{1}^{j}\right]$ sowie $\left[\left(p_{x}, p_{e}\right) e-p_{s} z\right]$ eine stetige Funktion auf $\mathrm{p}_{\times} \widehat{Y}^{j}$ definiert, zeigt man über die selben Argumente wie Debreu (1976), S. 59 f. die Behauptung des obigen Hilfssatzes.

$$
\text { Q.E.D. }
$$

In Analogie zu Nikaido (1968), S. 274 f. wird definiert $\mathrm{P}_{i} \equiv$ $\left\{p \in P \mid I_{i}(p)>0\right.$ und/oder $p_{n+l}>0$ mit $1=3$ für $i=1, \ldots, N_{1}$ und $l=4$ für $\left.i=N_{1}+1, \ldots, N\right\}$. Da proj $\left[1, \hat{x}^{i}\right]=\operatorname{proj}\left[1, \hat{x}^{j}\right]$ für $1=1, \ldots, n+4$ mit $i, j=1, \ldots, N$ gilt, können die Vereinbarungen getroffen werden:

$\hat{\mathrm{s}}_{1} \equiv \operatorname{proj}\left[\mathrm{n}+2+1, \hat{\mathrm{x}}^{\mathrm{i}}\right]$ mit $\mathrm{l}=1,2$ und $\mathrm{i}=1, \ldots, \mathrm{N}$ 


$$
\begin{aligned}
& \hat{S} \equiv \hat{s}_{1} \times \hat{s}_{2} \\
& \hat{x}_{L}^{i} \equiv \operatorname{proj}_{1}\left[1, \hat{x}^{i}\right] \times \ldots \times \operatorname{proj}\left[n+2, \hat{x}^{i}\right]
\end{aligned}
$$

Die individuellen Umweltbelastungsnachfragefunktionen $\widetilde{c}_{i}$ werden dadurch definiert, daß für $i=1, \ldots, N_{1}$ die Urbildbereiche der auf S. definierten Funktionen $\sigma_{i}$ durch $P_{i} \times \hat{S}_{2}$ und deren Bildbereiche durch $\hat{S}_{1}$ ersetzt werden. Analog wird $P_{i} \times \hat{S}_{1}$ als Urbildbereich und $\widehat{i}_{2}$ als Bildbereich der Funktionen $\widetilde{\sigma}_{i}$ mit $i=N_{1}+1, \ldots, N$ gewählt.

Lemma 6:

Die Funktionen $\tilde{o}_{i}: \mathrm{P}_{i} \times \hat{\mathrm{S}}_{2} \rightarrow \hat{\mathrm{S}}_{1}$ mit $\mathrm{i}=1, \ldots, \mathrm{N}_{1}$ und $\tilde{\sigma}_{i}: \mathrm{P}_{i} \times \hat{\mathrm{S}}_{1} \rightarrow \hat{\mathrm{S}}_{2}$ mit $i=N_{1}+1, \ldots, N$ sind stetig.

\section{Beweis :}

Im folgenden wird die stetigkeit der $i=1, \ldots, N_{1}$ Funktionen $\tilde{\sigma}_{i}$ gezeigt. Die Stetigkeit von $\tilde{\sigma}_{i}$ mit $i=N_{1}+1, \ldots, N$ zeigt man analog. Für die nachstehenden Ausführungen dieses Beweises gilt immer $i=1, \ldots, N_{1}$.

Zuerst wird die Stetigkeit der Budgetkorrespondenz $\widetilde{\mathrm{B}}_{i}: \mathrm{P}_{i} \times \widehat{\mathrm{S}}_{2} \rightarrow$ $\hat{s}_{1}$, definiert als $\widetilde{\mathrm{B}}_{i}\left(\mathrm{p}, \mathrm{s}_{2}\right)=\left\{\left(\mathrm{x}^{i}, \mathrm{~s}^{i}\right) \in \hat{\mathrm{x}}^{i} \mid \mathrm{s}_{2}^{i}=\mathrm{s}_{2}\right.$ und $\left(\mathrm{p}_{\mathrm{x}}, \mathrm{p}_{\mathrm{e}}\right)$ $\left.x^{i}-\beta_{i} p_{s} s^{i} \leqq I_{i}(p)\right\}$ gezeigt. Für $\left(p_{1}, s_{2}\right) \in P_{i} \times \widehat{S}_{2}$ ist die Existenz eines Punktes $\left(x^{i}, s^{i}\right) \in \hat{x}^{i}$ mit $\left(p_{x}, p_{e}\right) x^{i}-\beta_{i} p_{s} s^{i}<I_{i}(p)$ gesichert. Dies ist so, da für $p \in P_{i}$ und $I_{i}(p)>0$ der punkt $o \in \hat{x}^{i}$ die obige Ungleichung erfüllt. Für $I_{i}(p)=0$ und $p_{n+3}>0, s_{2} \in \hat{s}_{2}$ erfüllt der Punkt $\left(x^{i}, s^{i}\right) \in \hat{x}^{i}$ mit $x^{i}=0, s_{1}^{i}>0$ die Ungleichung. M.a.W. die Existenz eines "cheaper points" ist gewährleistet, womit für jedes $\left(p, s_{2}\right) \in P_{i} \times \hat{S}_{2}$ die Mengen $\widetilde{B}_{i}\left(p, s_{2}\right)$ ein nichtleeres Inneres haben.

Um die Oberhalb-Semistetigkeit von $\widetilde{B}_{i}$ zu zeigen, sei $\left(x^{n}, s^{n}\right) \in \widetilde{B}_{i}\left(p^{n}\right.$, $\left.s_{2}^{n}\right),\left(p^{n}, s_{2}^{n}\right) \rightarrow\left(p, s_{2}\right)$ und $\left(x^{n}, s^{n}\right) \rightarrow(x, s)$ mit $(x, s) \in \hat{x}^{i},\left(p, s_{2}\right) \in P_{i} \times \hat{S}_{2}$ vorausgesetzt. $(x, s) \notin \widetilde{B}_{i}\left(p, s_{2}\right)$ impliziert dann $\left(p_{x}, p_{e}\right) x-\beta_{i} p_{s} s>$ $I_{i}(p)$. Da $I_{i}(p),\left(p_{x}, p_{e}\right) x$ und $\beta_{i} p_{s} s$ stetige Funktionen sind, erhält man für genügend großes $n$ aber $\left(p_{x}^{n}, p_{e}^{n}\right) x^{n}-\beta_{i} p_{s}^{n} s^{n}>I_{i}\left(p^{n}\right)$ und 
$\left(x^{n}, s^{n}\right) \notin \widetilde{B}_{i}\left(p^{n}, s_{2}^{n}\right)$, womit offensichtlich $(x, s) \in \widetilde{B}_{i}\left(p, s_{2}\right)$ gilt.

Die Unterhalb-Semistetigkeit von $\widetilde{B}_{i}\left(p, s_{2}\right)$ ist gezeigt, wenn für $\left(p^{n}, s_{2}^{n}\right) \rightarrow\left(p, s_{2}\right),(x, s) \in \widetilde{B}_{i}\left(p, s_{2}\right)$ eine Folge $\left(x^{n}, s^{n}\right) \in \hat{x}^{i}$ derart gefunden werden kann, da $\left(x^{n}, s^{n}\right) \in \widetilde{B}_{i}\left(p^{n}, s_{2}^{n}\right)$ und $\left(x^{n}, s^{n}\right) \rightarrow(x, s)$ gilt. Da für jedes $\left(p, s_{2}\right) \in P_{i} \times \hat{s}_{2}$ ein cheaper point existiert, können analog zu Debreu (1979), S. 79 f. Folgen definiert werden, welche die eben genannte Eigenschaft besitzen.

Da die Nutzenfunktionen $u_{i}$ stetige, reellwertige Funktionen sind, $\hat{\mathrm{x}}^{i}$ kompakt ist und $\widetilde{\mathrm{B}}_{i}$ stetig ist, findet der Maximumsatz von Berge Anwendung, d.h. die individuellen Nachfragekorrespondenzen $\tilde{h}_{i}: P_{i} \times \widehat{S}_{2} \rightarrow \hat{X}^{i}$ sind oberhalb-semistetig. Da die Nutzenfunktionen $u_{i}$ streng quasi-konkav sind, besitzen sie über der konvexen, kompakten Menge $\widetilde{B}_{i}\left(p, s_{2}\right)$ genau ein Maximum, womit $\widetilde{h}_{i}\left(p, s_{2}\right)$ für alle $\left(p, s_{2}\right) \in P_{i} \times \widehat{s}_{2}$ genau ein Element enthält und im folgenden durch die Funktion. $\widetilde{h}_{i}$, definiert als $\tilde{h}_{i}\left(p, s_{2}\right)=\left\{\widetilde{h}_{i}\left(p, s_{2}\right)\right\}$ ersetzt wird. Da $\tilde{h}_{i}$ oberhalb-semistetig ist, folgt die stetigkeit der Funktionen $\tilde{h}_{i}$ und $\tilde{\sigma}_{i}$, definiert als $\tilde{\sigma}_{i}\left(p, s_{2}\right)=\operatorname{proj}[n+3$, $\left.\tilde{\mathrm{h}}_{i}\left(\mathrm{p}, \mathrm{s}_{2}\right)\right]$.

$$
\text { Q.E.D. }
$$

Sei $P^{\square} \equiv\{p \in P \mid p>0\}$ und $\bar{P}^{\square}$ die abgeschlossene Hülle von $P^{D}$. Da nach Annahme (c.3) $\omega^{i} \in R_{++}^{n}$ für jedes $i=1, \ldots, N$ gilt und nach Lemma $5 \hat{\pi}_{i}(p) \geqq 0$ für jedes $p \in P$, ist für $p>0$ auch $I_{i}(p)>0$ erfüllt, was $P \square \subset P_{i}$ impliziert. Aus $P D \subset P_{i} \subset P$ und $\bar{P} D=P$ folgt $\bar{P}_{i}=P$, wobei $\bar{P}_{i}$ die abgeschlossene Hüllle der Menge $P_{i}$ bezeichnet. Daher sind die Voraussetzungen der Theoreme 4.7 und 4.8 in Nikaido (1968), s. 72 erfüllt, womit für $i=1, \ldots, N_{1}$ eine Fortsetzung $\bar{\sigma}_{i}$ der Funktion $\tilde{\sigma}_{i}$ auf $\mathrm{P} \times \hat{\mathrm{S}}_{2}$ derart konstruiert werden kann, daB $\bar{\sigma}_{i}\left(p, s_{2}\right)=\tilde{\sigma}_{i}\left(p, s_{2}\right)$ für alle $\left(p, s_{2}\right) \in P_{i} \times \widehat{S}_{2}$ gilt und $\bar{\sigma}_{i}$ ein stetige Funktion ist mit $\bar{\sigma}_{i}\left(p, s_{2}\right) \in$ proj $\left[n+3, \hat{B}_{i}\left(p, s_{2}\right)\right]$. Analog zum eben Gesagten werden die $i=\mathrm{N}_{1}+1, \ldots, \mathrm{N}$ "modifizierten" individuellen Umweltbelastungsnachfragefunktionen $\bar{\sigma}_{i}\left(p, s_{1}\right)$ definiert. 
Lemma 7 :

Die Korrespondenz $\bar{\zeta}: \mathrm{P} \times \hat{\mathrm{S}}+\hat{\mathrm{S}}$, definiert als $\bar{\zeta}(\mathrm{p}, \mathrm{s})=$ $\underset{i=1, \ldots, N_{1}}{\operatorname{median}} \bar{\sigma}_{i}\left(p, s_{2}\right) \times \operatorname{median}_{i=N_{1}+1, \ldots, N} \bar{\sigma}_{i}\left(p, s_{1}\right)$ ist oberhalb-semistetig mit nichtleeren, konvexen Bildmengen $\bar{\zeta}(p, s)$ für jedes $(p, s) \in P \times \hat{S}$.

\section{Beweis :}

Aus Lemma 6 folgt wegen des oben Gesagten für jedes $\left(p_{1}, s_{2}\right) \epsilon$ $P \times \hat{S}_{2}$ und $i=1, \ldots, N_{1}$ auch $\bar{\sigma}_{i}\left(p, s_{2}\right) \neq \varnothing$. Analog ist für $i=N_{1}+1, \ldots, N$, $\left(p, s_{2}\right) \in P \times \hat{S}_{2}$ auch $\bar{\sigma}_{i}\left(p, s_{1}\right) \neq \varnothing$. Daher ist median $\bar{\sigma}_{i}\left(p, s_{2}\right)$ und $\operatorname{median}_{i=N_{1}+1, \ldots, N} \frac{2}{\sigma}_{i}\left(p, s_{1}\right)$ nichtleer.

Die Menge $\bar{\zeta}(p, s)$ ist als kartesisches produkt der Mengen $\underset{i=1, \ldots, N_{1}}{\operatorname{median}} \bar{\sigma}_{i}\left(p, s_{2}\right)$ und median $\bar{\sigma}_{i=N_{1}+1, \ldots, N}\left(p, s_{1}\right)$ konvex, wenn die genannten "Medianmengen" konvex sind. Sei $s_{1}^{\prime}, s_{1}^{\prime \prime} \in \underset{i=N_{1}+1, \ldots, N}{\operatorname{median}} \bar{\sigma}_{i}\left(p, s_{2}\right)$, $\mathbf{s}_{1}^{\prime}<\mathbf{s}_{1}^{\prime \prime}, \mathbf{s}_{1}^{\lambda}=\lambda \mathrm{s}_{1}^{\prime}+(1-\lambda) \mathrm{s}_{1}^{\prime \prime}$ und $0<\lambda<1$. Da $\mathbf{s}_{1}^{\prime}<\mathbf{s}_{1}^{\lambda}$ folgt

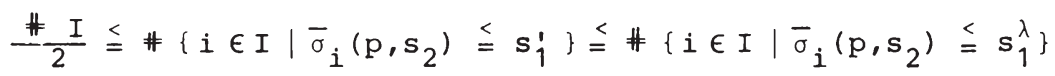

wobei $I \equiv\left\{1, \ldots, N_{1}\right\}$. Wegen $s_{1}^{\lambda}<s_{1}^{\prime \prime}$ folgt

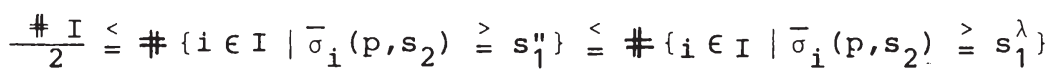

Daher ist $s_{1}^{\lambda} \in \underset{i=1, \ldots, N_{1}}{\operatorname{median}} \bar{\sigma}_{i}\left(p, s_{2}\right)$. Analog zeigt man die Konvexität der Menge mecian ${ }_{i=N_{1}+1, \ldots, N} \bar{\sigma}_{i}\left(p, s_{1}\right)$.

Da $\hat{S}_{1}, \hat{S}_{2}$ kompakte Mengen sind, ist die Korrespondenz $\bar{\zeta}$ als kartesisches produkt der Korrespondenzen median $\underset{i=1, \ldots, N_{1}}{\bar{\sigma}_{i}}$ und median $\underset{i=N_{1}+1, \ldots, N}{ } \vec{\sigma}_{i}$ oberhalb-semistetig, wenn die genannten "Median-Korrespondenzen" oberhalb-semistetig sind. Es wird die oberhalb-Semistetigkeit der Korresporidenz median $\bar{\sigma}_{i}$ gezeigt. Sei $\left(p^{n}, s_{2}^{n}\right) \rightarrow\left(\bar{p}_{1}, \bar{s}_{2}\right)$, $s_{1}^{n} \rightarrow \vec{s}_{1}, s_{1}^{n} \in \underset{j=1, \ldots, N_{1}}{\operatorname{median}} \bar{\sigma}_{i}\left(p^{n}, s_{2}^{n}\right)$ und $\bar{s}_{1} \notin \underset{i=1, \ldots, N_{1}}{\notin \operatorname{median}} \bar{\sigma}_{i}\left(\bar{p}_{1} \bar{s}_{2}\right)$ unter- 


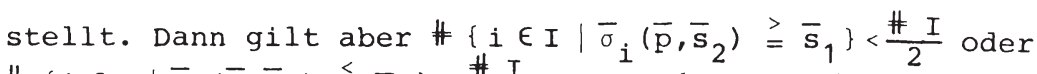
$\#\left\{i \in I \mid \bar{\sigma}_{i}\left(\bar{p}_{1}, \bar{s}_{2}\right) \leqq \bar{s}_{1}\right\}<\# \frac{I}{2} \operatorname{mit} I \equiv\left\{1, \ldots, N_{1}\right\}$.

Sei \# $\left\{i \in I \mid \bar{\sigma}_{i}\left(\frac{2}{p}, \bar{s}_{2}\right) \geqq \bar{s}_{1}\right\}<\frac{\# I}{2}$ unterstellt. Da die Funktionen $\bar{\sigma}_{i}$ stetig sind, folgt bei großem $n$ für jedes $i \in I$, welches

$$
\bar{\sigma}_{i}\left(p^{n}, s_{2}^{n}\right) \geqq s_{1}^{n} \text { erfüllt, auch } i \in\left\{i \in I \mid \bar{\sigma}_{i}\left(\bar{p}, \bar{s}_{2}\right) \geqq \bar{s}_{1}\right\} \text {. }
$$

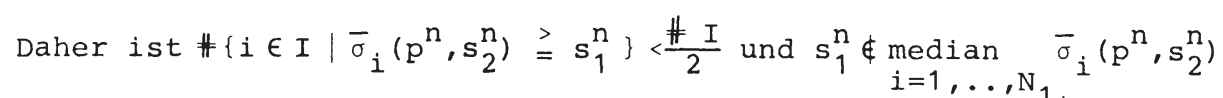

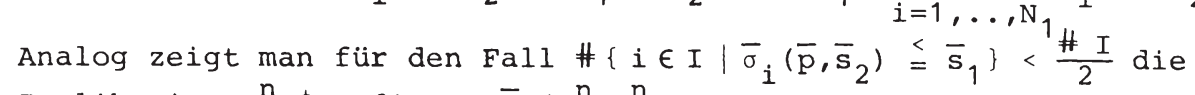
Implikation $s_{1}^{n} \notin$ median $\bar{\sigma}_{i=1, \ldots, N}\left(p^{n}, s_{2}^{n}\right)$, womit die Oberhalb-Semistetigkeit der Korrespondenz $\underset{i=1, \ldots, N_{1}}{ } \bar{\sigma}_{i}$ gezeigt ist.

$$
\text { Q.E.D. }
$$

Die $i=1, \ldots, N$ Nachfragekorrespondenzen nach privaten Gütern $\widehat{H}_{i}$ werden dadurch definiert, daß die Urbildbereiche der, durch Beziehung (7), (7a), S. . definierten Korrespondenzen $\mathrm{H}_{i}$ durch $\mathrm{P} \times \hat{\mathrm{S}}$ und deren Bildbereichedurch $\hat{\mathrm{X}}_{\mathrm{L}}^{\mathrm{i}}$ ersetzt werden. Dabei kann für $p \in P_{i}$ mit $p_{x}=0, p_{s}>0$ und $s \in \hat{S}$ mit $s=0$ für einige $i$ die bedingte Budgetkorrespondenz $\hat{B}_{i}^{b}: P \times \widehat{S} \rightarrow \widehat{X}_{L}^{i}$ im genannten Punkt $(p, s)$ unstetig sein. Dies läßt sich für den Fall eines privaten Gutes und eines Umweltbelastungsindikators anhand des nachstehenden Diagramms verdeutlichen.

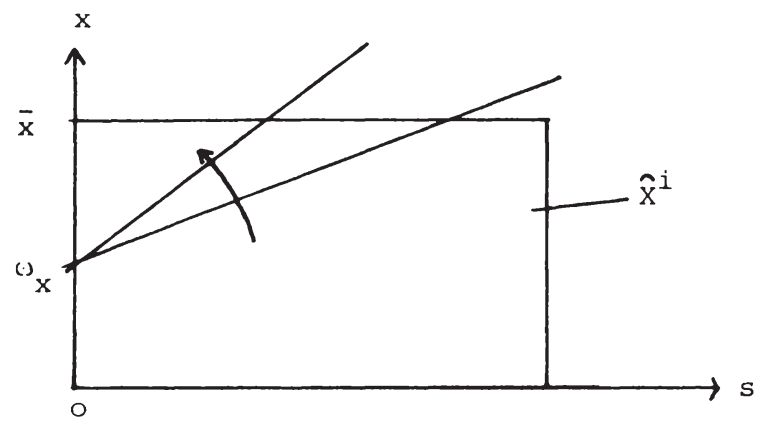

Schaubild 6 
Für $\mathrm{p}^{\mathrm{n}} \rightarrow \mathrm{p}, \mathrm{s}^{\mathrm{n}} \rightarrow \mathrm{s}$ mit $\mathrm{p} \equiv\left(\mathrm{p}_{\mathrm{x}}, \mathrm{p}_{\mathrm{s}}\right) \neq \mathrm{p}^{\mathrm{n}}, \mathrm{p}_{\mathrm{x}}=0, \mathrm{p}_{\mathrm{s}}=1$ und $\mathrm{s}^{\mathrm{n}}=\mathrm{s}$ $=$ o gilt in Schaubild $6\left[0, \omega_{x}\right]=\hat{B}_{i}^{b}\left(p^{n}, s^{n}\right)$ und $[0, \bar{x}]=\hat{B}_{i}^{b}(p, s)$, womit die bedingte Budgetkorrespondenz $\hat{B}_{1}^{b}$ im genannten Punkt $(p, s)$ unstetig ist. Aus diesem Grunde wird definiert

$$
\dot{\mathrm{P}}_{i} \equiv\left\{p \in \mathrm{P} \mid \hat{\mathrm{H}}_{i}(\mathrm{p}, \mathrm{s}) \text { ist stetig für } s \in \hat{\zeta}(p, s)\right\}
$$

Lemma 8:

$\mathrm{P}^{\mathrm{D}} \subset \stackrel{\circ}{\mathrm{P}}_{i}$ für jedes $i=1, \ldots, N$ und $\mathrm{P}_{i} \subset \stackrel{\circ}{\mathrm{P}}_{i}$ für ein $i \in\left\{1, \ldots, \mathrm{N}_{1}\right\}$ und ein $i \in\left\{N_{1}+1, \ldots, N\right\}$.

\section{Beweis :}

Da für jedes $(p, s) \in P \times \hat{S}$ die Menge $\hat{B}_{i}^{b}(p, s)$ kompakt ist und $u_{i}$ stetige Funktionen sind, folgt nach dem Weierstrass-Satz $\widehat{H}_{i}(p, s) \neq \varnothing$ für jedes $i$. Wegen der strengen Quasi-Konkavität der $i=1, \ldots, N$ Nutzenfunktionen $u_{i}$ besitzen diese über der konvexen, kompakten Menge $\hat{B}_{i}^{b}(p, s) \times\{s\}$ mit $s \in \hat{s}, \hat{B}_{i}^{b}(p, s) \subset \hat{X}_{L}^{i}$ genau ein Maximum, womit $\hat{H}_{i}(p, s)$ für alle $(p, s) \in P \times \widehat{S}$ genau ein Element enthält und $i m$ folgenden durch die Funktion $\widehat{\hat{H}}_{1}$, definiert als $\widehat{H}_{i}(p, s)=\left\{\widehat{H}_{i}(p, s)\right\}$ ersetzt wird.

Jetzt wird $P{ }^{{ }^{\prime}} \subset \stackrel{\circ}{P}_{i}$ für jedes $1=1, \ldots, N$ gezeigt. Sel $p \in P$ mit $p \equiv\left(p_{x}, p_{e}, p_{s}\right)$ und $p_{x} \geq 0$ unterstellt. Da wegen Annahme (c.3) für jedes $i=1, \ldots, N \quad \omega^{i} \in R_{++}^{n}$ gilt, ist $I_{1}(p)>0$ für jedes $1=1, \ldots, N$. Da $\hat{S} \subset R_{+}^{2}$ folgt $I_{i}(p)+\beta_{i} p_{s} s>0$, womit der punkt $x \in \hat{x}_{L}^{1}$ mit $x=0$ die Bedingung $\left(p_{x}, p_{e}\right) x<I_{i}(p)+\beta_{i} p_{s} s$ erfüllt. Daher zeigt man analog zum Beweis des Lemma 6 die stetigkeit der Funktion $\widehat{\hat{H}}_{i}$ für alle $(\mathrm{p}, \mathrm{s}) \in \stackrel{\mathrm{c}}{\mathrm{P}} \times \hat{\mathrm{S}}$, wobei $\stackrel{\infty}{\mathrm{P}} \equiv\left\{\mathrm{p} \in \mathrm{P} \mid \mathrm{p}_{\mathrm{X}} \geq 0\right\}$. Da $\mathrm{P}^{\mathrm{a}} \subset \mathbb{P}$ und $\stackrel{\infty}{\mathrm{P}} \subset \mathrm{P}_{i}$ ist, folgt die Behauptung $P^{{ }^{a} \subset \stackrel{P}{P}_{i}}$.

$P_{i} \subset \stackrel{\circ}{P}_{i}$ ist bewiesen, wenn für jedes $p \in P_{i}$ und $s \in \hat{\zeta}(p, s)$ die Stetigkeit der Funktion $\widehat{\mathrm{H}}_{i}$ gezeigt ist. Im folgenden wird $\mathrm{P}_{i} \subset \stackrel{\circ}{\mathrm{P}}_{1}$ für ein $i \in\left\{1, \ldots, N_{1}\right\}$ bewiesen. Zunächst ist wegen der eben genannten Argumente füs $i=1, \ldots, N_{1}$ die Funktion $\widehat{\widehat{H}}_{1}$ in jedem Punkt $(p, s) \epsilon$ $\left\{p \in P \mid I_{i}(p)>0\right\} \times \widehat{S}$ stetig. Da $p_{x}=0$ eine notwendige Bedingung für $I_{i}(p)=0$ ist, bleib̧t die Stetigkeit der Funktion $\widehat{\hat{H}}_{i}$ für 
ein $i \in\left\{1, \ldots, N_{1}\right\}$ in jedem punkt $(p, s)$ mit $p \in \hat{P} \equiv\left\{p \in P \mid p_{x}=0\right.$ und $\left.\mathrm{p}_{\mathrm{n}+3}>0\right\}, \mathrm{s} \equiv\left(\mathrm{s}_{1}, \mathrm{~s}_{2}\right), \mathrm{s}_{2} \in \hat{\mathrm{s}}_{2}, \mathrm{~s}_{1} \in \underset{i=1, \ldots, \mathrm{N}_{1}}{\operatorname{median}} \hat{\sigma}_{i}\left(\mathrm{p}_{1}, \mathrm{~s}_{2}\right) \mathrm{zu}$ prüfen. Für $p \in \hat{\mathrm{p}}, \mathrm{s}_{2} \in \hat{\mathrm{s}}_{2}$ folgt nach Annahme (c.2) $\hat{\sigma}_{i}\left(p, s_{2}\right)=0$ für jedes $i=1, \ldots, N_{1}$. Dies ist so, da $\left(p, s_{2}\right) \in \hat{p} \times \hat{S}_{2}$ für jedes $i=1, \ldots, N_{1} \hat{B}_{i}\left(p, s_{2}\right)=\hat{x}_{s}^{i}$ mit $\hat{x}_{s}^{i} \equiv \hat{x}_{L}^{i} \times \hat{S}_{1} \times\left\{s_{2}\right\}$ impliziert, womit $(\bar{x}, \bar{s}) \in \widehat{B}_{i}\left(p, s_{2}\right)$ mit $\bar{x}=\max \hat{x}_{L}^{i}$ und $\bar{s}=\left(0, s_{2}\right)$ gilt. Aufgrund der strengen Monotonie der Nutzenfunktionen gilt $u_{i}(\bar{x}, \bar{s})>$ $u_{i}(x, s)$ für alle $(x, s) \neq(\bar{x}, \bar{s})$ und $(x, s) \in \widehat{B}_{i}\left(p, s_{2}\right)$, was $\hat{\sigma}_{i}\left(p, s_{2}\right)=o$ für jedes $i=1, \ldots, N_{1}$ impliziert. Daher ist für $\left(p, s_{2}\right)$ $\epsilon \hat{\mathrm{P}} \times \widehat{\mathrm{S}}_{2}$ auch median $\hat{\sigma}_{i=1, \ldots, \mathrm{N}_{1}}\left(p, s_{2}\right)=0$. Da $\hat{\mathrm{P}} \subset \mathrm{P}_{i}$ sind nach Lemma 6 und 7 für jedes $\left(p, s_{2}\right) \in \hat{\mathrm{P}} \times \hat{\mathrm{s}}_{2}$ die Funktionen $\hat{\sigma}_{i}$ stetig und median $\hat{\sigma}_{i=1}$ oberhalb-semistetig. Da median $\hat{\sigma}_{i=1}\left(p, s_{2}\right)$ für jedes $i=1, \ldots, N_{1} i \quad i=1, \ldots, N_{1}$ $\left(p, s_{2}\right) \in \hat{\mathrm{P}} \times \hat{\mathrm{S}}_{2}$ genau ein Element enthält, folgt die stetigkeit der Korrespondenz median $\hat{\sigma}_{i}$ im Punkte $\left(p, s_{2}\right) \in \hat{\mathrm{p}} \times \widehat{\mathrm{S}}_{2}$. Betrach$i=1, \ldots, N_{1}$

ten wir die Folge $p^{n} \rightarrow \bar{p}, s^{n} \rightarrow \bar{s} \operatorname{mit} s_{1}^{n} \in \underset{i=1, \ldots, N_{1}}{\operatorname{median}} \hat{\sigma}_{i}\left(p^{n}, s_{2}^{n}\right)$, $\mathrm{s}_{2}^{\mathrm{n}}, \overline{\mathrm{s}}_{2} \in \hat{\mathrm{s}}_{2}, \mathrm{p}^{\mathrm{n}} \in \mathrm{P}_{i}, \overline{\mathrm{p}} \in \hat{\mathrm{p}}$. Wegen des eben Gesagten gibt es für hinreichend großes $n \geq \bar{n}$ ein $d \in\left\{1, \ldots, N_{1}\right\}$ derart, daß $\hat{\sigma}_{d}\left(p^{n}, s_{2}^{n}\right)$

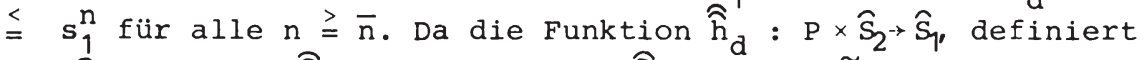
als $\widehat{h}_{d}\left(p, s_{2}\right)=\left\{\widehat{h}_{d}\left(p, s_{2}\right)\right\}$, wegen $\widehat{h}_{d}\left(p, s_{2}\right)=\widetilde{h}_{d}\left(p, s_{2}\right)$ für $\left(p, s_{2} \epsilon\right.$ $\mathrm{P}_{\mathrm{d}} \times \widehat{\mathrm{S}}_{2}$ nach Lemma 6 stetig über $\mathrm{P}_{\mathrm{d}} \times \widehat{\mathrm{S}}_{2}$ ist und $\widehat{\mathrm{H}}_{\mathrm{d}}\left(\mathrm{p}, \widehat{\sigma}_{\mathrm{d}}\left(\mathrm{p}, \mathrm{s}_{2}\right), \mathrm{s}_{2}\right)=$ $\operatorname{proj}\left[1, \widehat{h}_{d}\left(p, s_{2}\right)\right] \times \ldots \times \operatorname{proj}\left[n+2, \widehat{h}_{d}\left(p, s_{2}\right)\right]$ ist, gilt $\lim _{n \rightarrow \infty} \widehat{\widehat{H}}_{d}\left(p^{n}\right.$, $\left.\hat{\sigma}_{d}\left(p^{n}, s_{2}^{n}\right), s_{2}^{n}\right)=\widehat{H}_{d}\left(\bar{p}, \hat{\sigma}_{d}\left(\bar{p}, \bar{s}_{2}\right), \bar{s}_{2}\right)$. Wegen des oben Gesagten ist dabei $\widehat{\hat{H}}_{\mathrm{d}}\left(\overline{\mathrm{p}}, \hat{\sigma}_{\mathrm{d}}\left(\overline{\mathrm{p}}, \overline{\mathrm{s}}_{2}\right), \overline{\mathrm{s}}_{2}\right)=\max \hat{\mathrm{x}}_{\mathrm{L}}^{\mathrm{d}}$. Weiterhin impliziert $s_{1}^{n} \geq \hat{\sigma}_{d}\left(p^{n}, s_{2}^{n}\right)$ auch $I_{d}\left(p^{n}\right)+\beta_{d} p_{s}^{n} s^{n} \stackrel{>}{=} I_{d}\left(p^{n}\right)+\beta_{d} p_{s}^{n}\left[\hat{\sigma}_{d}\left(p^{n}, s_{2}^{n}\right)\right.$, $\left.s_{2}^{n}\right]$, womit $\widehat{B}_{d}^{b}\left(p^{n}, \hat{\sigma}_{d}\left(p^{n}, s_{2}^{n}\right), s_{2}^{n}\right) \subset \hat{B}_{d}^{b}\left(p^{n}, s^{n}\right)$, was $\widehat{H}_{d}\left(p^{n}, s^{n}\right) \geqslant$ $\widehat{\widehat{H}}_{d}\left(p^{n}, \widehat{\sigma}_{d}\left(p^{n}, s_{2}^{n}\right)\right.$ für jedes $n=\frac{2}{n}$ impliziert. Daher ist $\lim _{n \rightarrow \infty} \widehat{H}_{d}\left(p^{n}\right.$, $\left.s_{-}^{n}\right) \geqq \lim _{n \rightarrow \infty} \widehat{H}_{d}\left(p^{n}, \hat{\sigma}_{d}\left(p^{n}, s^{n}, s_{2}^{n}\right)=\max \widehat{x}_{L}^{d}\right.$. Da aber $\widehat{H}_{d}\left(p^{n}, s^{n}\right) \leqq \max$

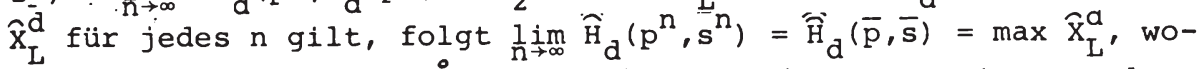
mit die Behauptung $P_{i} \subset \stackrel{\circ}{P}_{i}$ für $d \in\left\{1, \ldots, N_{1}\right\}$ bewiesen ist. Analog zeigt man die Gültigkeit der Behauptung $P_{i} \subset \stackrel{\circ}{P}_{i}$ für $d \in\left\{N_{1}+1, \ldots, N\right\}$. 
Analog zur Definition der Funktion $\widetilde{\sigma}_{i}$ wird jetzt $\widetilde{\mathrm{H}}_{i}$ dadurch definiert, daß die Urbildbereiche der auf S. Beziehung (7) definierten Korrespondenz $\mathrm{H}_{i}$ durch $\stackrel{\circ}{\mathrm{P}}_{i} \times \hat{\mathrm{S}}$ und deren Bildbereiche durch $\hat{\mathrm{x}}_{\mathrm{L}}^{i}$ ersetzt werden. Wegen des vorstehenden Hilfssatzes ist $\widetilde{\mathrm{H}}_{i}$ stetig über: $\stackrel{\circ}{\mathrm{P}}_{i} \times \hat{\mathrm{S}}$, wobei $\mathrm{P}^{\square} \subset \stackrel{\circ}{\mathrm{P}}_{i} \subset \mathrm{P}$ gilt. Daher sind die Voraussetzungen der Theoreme 4.7 und 4.8 in Nikaido (1968), S.7.2 erfüllt, womit eine Fortsetzung $\overline{\mathrm{H}}_{i}$ der Funktion $\widetilde{\mathrm{H}}_{i}$ auf $\mathrm{P} \times \hat{\mathrm{S}} \operatorname{der}-$ art konstruiert werden kann, daß $\overline{\mathrm{H}}_{i}$ stetig über $\mathrm{P} \times \widehat{\mathrm{S}}$ ist und $\bar{H}_{i}(p, s) \in \widehat{B}_{i}(p, s)$ für alle $(p, s) \in P \times \widehat{S}$ erfüllt. Da für jedes $i=1, \ldots, N \bar{H}_{i}$ über $P \times \widehat{S}$ stetig ist, ist auch $\bar{H}=\Sigma \bar{H}_{i}$ stetig über $\mathrm{P} \times \hat{\mathrm{S}}$.

Damit sind alle Voraussetzungen des zu formulierenden Fixpunktsatzes erfüllt.

\section{Teil: Fixpunktsatz}

Zunächst werden die Mengen $\hat{X}, \hat{\mathrm{Y}}^{\mathrm{K}+1}$ und $\mathrm{V}$ definiert als $\widehat{\mathrm{X}} \equiv \widehat{\mathrm{X}}_{\mathrm{L}} \times \hat{\mathrm{S}}$ mit $\widehat{\mathrm{X}}_{\mathrm{L}} \equiv \Sigma \hat{\mathrm{X}}_{\mathrm{L}}^{\mathrm{i}}, \widehat{\mathrm{Y}}^{\mathrm{K}+1} \equiv \operatorname{proj}\left[1, \widehat{\mathrm{Y}}^{\mathrm{K}+1}\right] \times \ldots \times \operatorname{proj}\left[\mathrm{n}+4, \widehat{\mathrm{Y}}^{\mathrm{K}+1}\right]$ , $\mathrm{V} \equiv\left\{\mathrm{v} \mid \mathrm{v}=\mathrm{y}^{+}\left(\Sigma \omega^{\mathrm{i}}, 0,0\right)\right.$ mit $\left.\mathrm{y} \in \hat{\mathrm{Y}} \equiv \Sigma_{\Sigma}^{\mathrm{K}} \hat{\mathrm{Y}}^{\mathrm{j}}\right\}$. Weiterhin werden Uberschußnachfragen definiert als $j=1$

$$
\begin{array}{lll}
u_{1}=\Sigma_{i} \cdot x_{1}^{i}-\Sigma_{j} y_{1}^{j}-\Sigma_{i} \omega_{1}^{i}-e_{1} & \text { für } 1=1, \ldots, n \\
u_{1}=\Sigma_{j} y_{1}^{j}-e_{1}+\Sigma_{i} x_{1}^{i} & \text { für } 1=n+1, n+2 \\
u_{1}=z_{1-n-2}-s_{1-n-2} & \text { für } 1=n+3, n+4
\end{array}
$$

Dann wird die Fixpunktkorrespondenz $\Gamma: P \times \hat{X} \times V \times \widehat{\hat{Y}}^{\mathrm{K}+1} \rightarrow \mathrm{P} \times \hat{\mathrm{X}} \times \mathrm{V} \times$ $\widehat{\widehat{Y}}^{K+1}$ definiert als das kartesische produkt der folgenden Korrespondenzen

$$
\begin{aligned}
& x^{\prime} \in \bar{H}(p, s) \\
& s^{\prime} \in \bar{\zeta}(p, s) \\
& v^{\prime} \in \hat{g}(p)+(\Sigma \omega t, 0,0) \\
& \left(e^{\prime}, z^{\prime}\right) \in \hat{F}(p) \\
& p_{i}^{\prime}=\frac{p_{1}+\max \left(u_{1}, 0\right)}{1+\sum_{l+1}^{n+4} \max \left(u_{1}, 0\right)} \quad \text { für } l=1, \ldots, n+4
\end{aligned}
$$


Lemma 9 :

Es existiert ein $\hat{a} \in \Gamma(\hat{a}) \operatorname{mit} \hat{a} \equiv(\hat{p}, \hat{x}, \hat{\mathbf{s}}, \hat{v}, \hat{e}, \hat{z})$

Beweis :

Die Menge $\mathrm{P} \times \widehat{\mathrm{X}} \times \mathrm{V} \times \widehat{\mathrm{Y}}^{\mathrm{K}+1}$ ist als kartesisches produkt nichtleerer, kompakter, konvexer Mengen ebenfalls nichtleer, kompakt und konvex. Weiterhin weist $\Gamma$ als produkt nichtleerer, oberhalb-semistetiger Korrespondenzen mit konvexen Bildmengen ebenfalls diese Eigenschaften aus. Aufgrund der Konstruktion der einzelnen "Teil"-Korrespondenzen gilt:

$x^{\prime} \in \bar{H}(p, s), s^{\prime} \in \bar{\zeta}(p, s),\left(e^{\prime}, z^{\prime}\right) \in \hat{F}(p)$ impliziert $\left(x^{\prime}, s^{\prime}\right) \in \hat{x}$, $\left(e^{\prime}, z^{\prime}\right) \in \hat{Y}^{K+1}$ und $y^{\prime} \in \hat{g}(p)$ impliziert $y^{\prime} \in \hat{Y}$, so daß auch $v^{\prime} \in V$.

Da ${ }_{\Sigma}^{n+4} p_{1}=1$ folgt ${ }_{\Sigma}^{n+4} p_{i}^{\prime}=1$ und $p^{\prime} \in P$. Damit ist für beliebiges $l=1 \quad l=1$

$\left(p^{\prime}, x^{\prime}, s^{\prime}, v^{\prime}, e^{\prime}, z^{\prime}\right)$ aus $\Gamma(p, x, s, v, e, z)$ auch $\left(p^{\prime}, x^{\prime}, s^{\prime}, v^{\prime}, e^{\prime}, z^{\prime}\right)$

$\epsilon \mathrm{P} \times \hat{\mathrm{X}} \times \mathrm{V} \times \hat{\mathrm{Y}}^{\mathrm{K}+1}$. Deshalb sind die Voraussetzungen für Kakutani's Fixpunktsatz erfüllt, $d . h$. der Punkt $(\hat{p}, \hat{x}, \hat{s}, \hat{v}, \hat{e}, \hat{z})$ existiert.

Q.E.D.

4. Teil: Der Fixpunkt ist ein Markt- und Abstimmungsgleichgewicht der ökonomie $\hat{E}$

$\operatorname{Da}(\hat{\mathrm{p}}, \hat{\mathrm{x}}, \hat{\mathrm{s}}, \hat{\mathrm{v}}, \hat{\mathrm{e}}, \hat{z})$ ein Fixpunkt ist, folgt wegen der Konstruktion $\operatorname{der} \operatorname{Korrespondenz} \Gamma$ auch $\hat{\mathbf{s}} \in \bar{\zeta}(\hat{\mathrm{p}}, \hat{\mathrm{s}}),(\hat{\mathrm{e}}, \hat{z}) \in \hat{\mathrm{F}}(\hat{\mathrm{p}}), \hat{\mathrm{x}} \in \overline{\mathrm{H}}(\hat{\mathrm{p}}, \hat{\mathrm{s}}), \hat{\mathrm{y}} \in \hat{\mathrm{g}}$ $(\hat{y})$. Wegen $\hat{y} \in \hat{g}(\hat{p})$ gibt es für jedes $j=1, \ldots, K$ ein $\hat{y}^{j} \in \hat{g}_{j}(\hat{p})$, womit die Bedingung (b) der Definition 3 für ökonomie $\hat{E}$ erfüllt ist. Analog gibt es wegen $\hat{\mathrm{x}} \in \overline{\mathrm{H}}(\hat{\mathrm{p}}, \hat{\mathrm{s}})$ für jedes $i$ ein $\hat{\mathrm{x}}^{i} \in \overline{\mathrm{H}}_{i}(\hat{\mathrm{p}}, \hat{\mathrm{s}})$. Da für jedes $i \omega^{i} \in R_{++^{\prime}}^{n}$ ist $\left\{p \in P \mid p_{x} \geq 0\right\} \subset\left\{p \in P \mid I_{i}(p)>0\right.$ für $i=1, \ldots, N\}$. Wegen der Definition der $\bar{H}_{i}$ ist daher für $\hat{p} \in\{p \in P \mid$ $\left.\mathrm{p}_{\mathrm{x}} \geq 0\right\} \cdot \overline{\mathrm{H}}_{i}(\hat{\mathrm{p}}, \hat{\mathrm{s}})=\widetilde{\mathrm{H}}_{i}(\hat{\mathrm{p}}, \hat{\mathrm{s}})$ für jedes $i$ erfüllt und es gilt $\hat{\mathrm{x}}^{\hat{i}} \in \hat{\mathrm{H}}_{i}(\hat{\mathrm{p}}, \hat{\mathrm{s}})$. Analog ist wegen der Definition der $\bar{\sigma}_{i}$ für $\hat{\mathrm{p}} \in\{\mathrm{p} \in \mathrm{P} \mid$ $\left.p_{x} \geq 0\right\}$ auch $\bar{\sigma}_{i}\left(\hat{p}, \hat{s}_{k(i)}\right)=\tilde{\sigma}_{i}\left(\hat{p}, \hat{s}_{k(i)}\right)$ erfüllt und per Definition folgt $\hat{s} \in \hat{\zeta}(\hat{p}, \hat{s})$, womit für ökonomie $\hat{E}$ die Bedingungen (a),

(d) der Definition 3 erfüllt sind. 
Jetzt wird $\hat{\mathrm{p}} \in\left\{\mathrm{p} \in \mathrm{P} \mid \mathrm{p}_{\mathrm{x}} z 0\right\}$ gezeigt. Sei $\hat{\mathrm{p}}_{\mathrm{x}}=0$ unterstellt. Da $\hat{p} \in \mathrm{P}$ gilt dann $\hat{\mathrm{p}}_{\mathrm{e}} \geq 0, \hat{\mathrm{p}}_{\mathrm{s}}=0$ oder $\hat{\mathrm{p}}_{\mathrm{e}}=0, \hat{\mathrm{p}}_{\mathrm{K}} \geq 0$ oder $\hat{\mathrm{p}}_{\mathrm{e}} \geq^{\geq 0}, \hat{\mathrm{p}}_{\mathrm{s}} \geq 0$. Für $\hat{\mathrm{p}}_{\mathrm{e}} \mathrm{Z}^{\geq}, \hat{\mathrm{p}}_{\mathrm{s}}=0$ ist aber $\hat{\pi}^{\mathrm{K}+1}>0,{ }^{K+1}$ womit $\Sigma_{i} I_{i}(\hat{\mathrm{p}})$ $>0$. Deshalb j.st für mindestens ein $d \in\{1, \ldots, N\}$ auch $I_{d}(\hat{p})>0$, womit wegen Lemma $8 \hat{x}_{d} \in \bar{H}_{d}(\hat{p}, \hat{s})$ durch $\hat{x}_{d} \in \tilde{H}_{d}(\hat{p}, \hat{s})$ ersetzt werden kann. Da nach Annahme (c.2) der Konsument d für jedes der Güter $1=1, \ldots, n$ eine strenge Präferenz besitzt, kann ein $\lambda>0$ gefunden werden, so daß

$$
\mathrm{x}^{\mathrm{d} \lambda} \equiv\left(\hat{\mathrm{x}}_{1}^{\mathrm{d}}+\lambda, \ldots, \hat{\mathrm{x}}_{\mathrm{n}}^{\mathrm{d}}+\lambda, \hat{\mathrm{x}}_{\mathrm{n}+1}^{\mathrm{d}}, \hat{\mathrm{x}}_{\mathrm{n}+2}^{\mathrm{d}}\right)>\hat{\mathrm{x}}^{\mathrm{m}} \equiv\left(\hat{\mathrm{x}}_{1}^{\mathrm{d}}, \ldots, \hat{\mathrm{x}}_{\mathrm{n}+1}^{\mathrm{d}}, \hat{\mathrm{x}}_{\mathrm{n}+2}^{\mathrm{d}}\right)
$$

und $\left(x^{d \lambda}, \hat{s}\right) \in x^{d}$ ist. Sei dann $x(t)$ der Punkt $(1-t) \hat{x}^{d}+t \hat{x}^{d \lambda}$, wobei $0<t<1$. Für jedes solches $t$ gilt zunächst $u_{d}(x(t), \hat{s})>u_{d}\left(\hat{x}^{d}\right.$, $\left.\hat{s}^{d}\right)$ und $\left(\hat{p}_{x}, \hat{p}_{\epsilon !}\right) x(t) \leqq I_{d}(\hat{p})+\beta_{d} \hat{p}_{s} \hat{s}$. Für $t$ nahe genug be $i$ null ist $(x(t), \hat{s})$ im Würfel $L$ enthalten, denn gemäß der Definition von $L, S$, ist $\left(\hat{x}^{d}, \hat{s}\right)$ ein innerer Punkt von L. Dies steht aber im Widerspruch $z u \hat{x}^{d} \in \bar{H}_{d}(\hat{p}, \hat{s})$, was $\hat{p}_{x}>0$ impliziert. Für $\hat{\mathrm{p}}_{\mathrm{e}}=0, \hat{\mathrm{p}}_{\mathrm{s}} \geq 0$ folgt wegen Lemma 8 für mindestens ein $i \in\{1, \ldots$ $\ldots, N\} \hat{x}^{i} \in \widetilde{H}_{i}(\hat{p}, \hat{s})$. Daher zeigt man analog zu den eben gemachten Ausführungen $\hat{\mathrm{p}}_{\mathrm{x}}>0$. Für $\hat{\mathrm{p}}_{\mathrm{e}} \geq 0, \hat{\mathrm{p}}_{\mathrm{s}} \geq 0$ gilt das eben Gesagte. Analog zum obigen Fall folgert man auch $\hat{p} \in\left\{\mathrm{p} \in \mathrm{P} \mid \mathrm{p}_{\mathbf{x}} \geq 0\right\}$ impliziert $\hat{\mathrm{p}}_{\mathrm{x}}>0$.

Zu zeigen bleibt, daß der Fixpunkt den Bedingungen (c.1) und (c.2) der Definition 3 für ökonomie $\widehat{E}$ genügt. Aufgrund der Konstruktion der Korrespondenz $\Gamma$ gilt

$$
\hat{\mathrm{p}}_{1}=\frac{\hat{\mathrm{p}}_{1}+\max \left(\hat{\mathrm{u}}_{1}, 0\right)}{1+\frac{\mathrm{r}}{1} \max \left(\hat{\mathrm{u}}_{1}, 0\right)} \quad \text { für } 1=1, \ldots, \mathrm{n}+4 \text { oder }
$$

umgeformt $\hat{p}_{1} \Sigma \max \left(\hat{u}_{1}, 0\right)=\max \left(\hat{u}_{1}, 0\right)$ für $1=1, \ldots, n+4$. Multipliziert man jede dieser $n+4$ Gleichungen mit $\hat{u}_{1}$ und summiert über 1 folgt

$$
\left[\sum_{1} \max \left(\hat{\mathrm{u}}_{1}, 0\right)\right] \sum_{1} \hat{\mathrm{p}}_{1} \hat{\mathrm{u}}_{1}=\sum_{1}\left[\max \left(\hat{\mathrm{u}}_{1}, 0\right)\right]^{2}
$$

Da $\hat{\mathrm{p}} \in\left\{\mathrm{p} \in \mathrm{P} \mid \mathrm{p}_{\mathrm{x}}>0\right\}$ gilt und wegen Annahme (c.2) alle Konsumenten in Bezug auf die Güter $1=1, \ldots, n$ unersättlich sind, kann das 
Ungleichheitszeichen in Beziehung (7a) Im Fixpunkt durch die Gleichheit zwischen Ausgaben und Einnahmen der Konsumenten ersetzt werden. Die Summation über die Budgetrestriktion aller Konsumenten ergibt daher

$$
\left(\hat{\mathrm{p}}_{\mathrm{x}}, \hat{\mathrm{p}}_{\mathrm{e}}\right) \hat{\mathrm{x}}=\hat{\mathrm{p}}_{\mathrm{x}} \omega+\sum_{j=1}^{K+1} \hat{\pi}_{j}(\hat{\mathrm{p}})+\hat{\mathrm{p}}_{\mathrm{s}} \hat{\mathrm{s}} \quad \text { mit } \hat{\mathrm{x}}=\Sigma \hat{\mathrm{x}}^{i}, \omega=\Sigma \omega{ }^{i}
$$

Aufgrund der Definition der Gewinnfunktionen der $j=1, \ldots, k+1$ Produzenten folgt

$$
\begin{aligned}
& \sum_{j}^{K+1} \hat{\pi}_{j}(\hat{p})=\sum_{l=1}^{n} \hat{p}_{1} \hat{y}_{1}-\sum_{l=n+1}^{n+2} \hat{p}_{1} \hat{y}_{1}+\sum_{l=1}^{n+2} \hat{p}_{1} \hat{e}_{1}-\hat{p}_{s} \hat{z} \\
& \text { mit } \hat{y}_{1}=\sum_{j=1}^{K} \hat{y}_{1}^{j}
\end{aligned}
$$

Deshalb kann (9) geschrieben werden als

$$
\begin{aligned}
& \sum_{1=1}^{n} \hat{\mathrm{p}}_{1}\left(\hat{\mathrm{x}}_{1}-\hat{\mathrm{y}}_{1}-\omega_{1}-\hat{\mathrm{e}}_{1}\right)+\sum_{1=n+1}^{\mathrm{n}+2} \hat{\mathrm{p}}_{1}\left(\hat{\mathrm{y}}_{1}-\hat{\mathrm{e}}_{1}+\hat{\mathrm{x}}_{1}\right)+\sum_{1=n+3}^{\mathrm{n}+4} \hat{\mathrm{p}}_{1} \\
& \left(\hat{\mathrm{z}}_{1-\mathrm{n}-2}-\hat{\mathrm{s}}_{1-\mathrm{n}-2}\right)=0
\end{aligned}
$$

womit wegen der Definition der $u_{1}$ folgt

$$
\sum_{1} \hat{\mathrm{p}}_{1} \hat{\mathrm{u}}_{1}=0
$$

Da per Definition $\Sigma_{1} \max \left(\hat{\mathrm{u}}_{1}, 0\right) \stackrel{0}{=}$ folgt wegen (10) aus (8) $\Sigma_{1}$ $\left[\max \left(\hat{\mathrm{u}}_{1}, 0\right)\right]^{2}=0$, womit $\hat{\mathrm{u}}_{1} \stackrel{\mathrm{s}}{=}$ für jedes $1=1, \ldots, \mathrm{n}+4$ gilt. Desweiteren ist wegen (10), $\hat{\mathrm{u}}_{1} \stackrel{\leq}{=}$ für jedes 1 und $\hat{\mathrm{p}} \in \mathrm{P}$ auch $\hat{\mathrm{p}}_{1} \hat{\mathrm{u}}_{1}=$ ○ für $1=1, \ldots, n+4$.

$\mathrm{Zu}$ zeigen bleibt $\hat{\mathrm{u}}_{1}=0$ für jedes 1 . Für $\hat{\mathrm{p}}>0$ ist dies wegen des eben Gesagten erfüllt. Da $\hat{p}_{x}>0$ ist $\hat{u}_{1}=0$ für $1=1, \ldots, n$ gezeigt. Sei $\hat{p}_{n+3}=0$ unterstellt. Da die Nutzenfunktionen $u_{i}$ streng monoton fallend in s sind, impliziert $\hat{\mathrm{p}}_{\mathrm{x}}>0, \hat{\mathrm{p}}_{\mathrm{n}+3}=0, \tilde{\sigma}_{i_{<}}\left(\hat{\mathrm{p}}_{\mathrm{s}} \hat{\mathrm{s}}_{2}\right)=0$ für $i=1, \ldots, N_{1}$, womit $\hat{s}_{1}=0$ folgt. Da $\hat{z}_{1} \in R_{+}$und $\hat{u}_{1} \leqq 0$ für jedes 1 , ist $\hat{\mathrm{z}}_{1}=0$, womit wegen $(\hat{\mathrm{e}}, \hat{\mathrm{z}}) \in \hat{\mathrm{Y}}^{\mathrm{K}+1}$ auch $\hat{\mathrm{e}}_{\mathrm{n}+1}=0$ folgt. Aus $\hat{e}_{n+1}=0, \hat{y}_{n+1} \in R_{+}, \hat{x}_{n+1}=0$ erhält man wegen $\hat{u}_{1} \leqq 0$ dann $\hat{\mathrm{u}}_{\mathrm{n}+1}=\hat{\mathrm{u}}_{\mathrm{n}+3}=0$. Analog zeigt man $\hat{\mathrm{u}}_{\mathrm{n}+2}=\hat{\mathrm{u}}_{\mathrm{n}+4}$ für $\hat{\mathrm{p}}_{\mathrm{n}+4}=0$. Für 
den Fall $\hat{\mathrm{p}}_{\mathrm{n}+1}=0, \hat{\mathrm{p}}_{\mathrm{n}+3}>0$ ist die gewinnmaximale Emissionsnachfrage des Umweltproduzenten $\hat{e}_{n+1}=0$ und wegen $\hat{u}_{n+1} \leqq 0$, $\hat{\mathrm{y}}_{\mathrm{n}+1} \in \mathrm{R}_{+}$folgt $\hat{\mathrm{u}}_{\mathrm{n}+1}=0$. Analoges gilt für $\hat{\mathrm{p}}_{\mathrm{n}+2}=0, \hat{\mathrm{p}}_{\mathrm{n}+4}>0$. womit $\hat{u}_{1}=0$ für $l=1, \ldots, n+4$ gezeigt ist.

5. Teil: Der Fixpunkt ist ein Markt- und Abstimmungsgleichgewicht der ökonomie E.

$\operatorname{Da}\left[\left(\hat{\mathrm{x}}^{\mathrm{i}}, \hat{\mathrm{s}}\right),\left(\hat{\mathrm{y}}^{\mathrm{j}}\right),(\hat{\mathrm{e}}, \hat{\mathrm{z}})\right]$, ein erreichbarer zustand der ökonomie $\hat{E}$ ist, liegt jedes $\left(\hat{x}^{i}, \hat{s}\right),\left(\hat{y}^{j}\right),(\hat{e}, \hat{z})$ im Inneren des würfels L.

(i) $\hat{y}^{j} \in g_{j}(\hat{p}), \quad(\hat{e}, \hat{z}) \in F \quad(\hat{p})$

Sei $y^{j} \in Y^{j}$ mit $\underset{l=1}{n} \hat{p}_{1} Y_{1}^{j}-\underset{l=n+1}{n+2} \hat{p}_{1} y_{1}^{j}>\sum_{l=1}^{n_{1}} \hat{p}_{1} \hat{y}_{1}^{j}-\sum_{l=n+1}^{n+2} \hat{p}_{1} \hat{y}_{1}^{j}$

unterstellt. Dann gilt

$\sum_{1=1}^{n} \hat{\mathrm{p}}_{1} \tilde{y}_{1}^{j}-\sum_{1==\mathrm{n}+1}^{\mathrm{n}+2} \hat{\mathrm{p}}_{1} \tilde{y}_{1}^{j}>\sum_{1=1}^{\mathrm{n}} \hat{\mathrm{p}}_{1} \hat{\mathrm{y}}_{1}^{j}-\sum_{1=\mathrm{n}+1}^{\mathrm{n}+2} \hat{\mathrm{p}}_{1} \hat{\mathrm{Y}}_{1}^{j} \quad$ für jedes $\tilde{y}^{j}=\lambda \hat{y}^{j}+$ $(1-\lambda) y^{j}$ mit $0<\lambda<1$. Wegen der Konvexität der $\mathrm{Y}^{j}$ ist dabei $\tilde{Y}^{j} \epsilon$ $\mathrm{Y}^{j}$. Außerdem ist für $\lambda$ nahe genug an null der Punkt $\tilde{y}^{j}$ in würfel L enthalten, denn $\hat{y}^{j}$ ist ein innerer Punkt von L. Folglich ist $\tilde{y}^{j} \in Y^{j} \cap L$, wass im Widerspruch $z u \hat{y}^{j} \in \hat{g}_{j}(\hat{p})$ steht. Analog zeigt $\operatorname{man}(\hat{\mathrm{e}}, \hat{\mathrm{z}}) \in \mathrm{F}(\hat{\mathrm{p}})$.

(ii) $\hat{\mathrm{x}}^{\mathrm{i}} \in \mathrm{H}_{\mathrm{i}}(\hat{\mathrm{p}}, \hat{\mathrm{s}})$

Sei $\left(x^{i}, \hat{s}\right) \in x^{i}$ derart, daß $\left(\hat{p}_{x}, \hat{p}_{e}\right) x^{i} \leqq I_{i}(\hat{p})+\beta_{i} \hat{p}_{s} \hat{s}$ und $u_{i}\left(x^{i}, \hat{s}\right)>u_{i}\left(\hat{x}^{i}, \hat{s}\right)$ gilt. Wegen Annahme (c.2) gilt dann $u_{i}\left(\widetilde{x}^{i}, \hat{s}\right)>$ $u_{i}\left(\hat{x}^{i}, \hat{s}\right)$ für $\tilde{x}^{i}=\lambda \hat{x}^{i}+(1-\lambda) x^{i}$ mit $0<\lambda<1$. Da $x^{i}$ konvex ist, gilt $\left(\tilde{x}^{i}, \hat{s}\right) \in x^{i}$ und $\tilde{x}^{i}$ erfüllt die Budgetrestriktion des Konsumenten $i$. Weiterhin ist $\left(\hat{x}^{i}, \hat{s}\right)$ ein innerer Punkt des würfels $L$, so daß für $\lambda$ nahe genug an null $\left(\tilde{x}^{i}, \hat{s}\right) \in x^{i} \cap L$ gilt. Dies steht aber im Widerspruch $z u \hat{x}^{i} \in \hat{H}_{i}(\hat{p}, \hat{s})$.

\section{(iii) $\hat{\mathbf{s}} \in \zeta(\hat{p}, \hat{\mathbf{s}})$}

Zunächst gilt $\left(\bar{x}^{i}, \bar{s}_{1}^{i}, \hat{s}_{2}\right)=\hat{h}_{i}\left(\hat{p}, \hat{s}_{2}\right)$ für $i=1, \ldots, N_{1}$. Damit ist auch $\left(\bar{x}^{i}, \bar{s}_{1}^{i}, \hat{s}_{2}\right)=h_{i}\left(\hat{p}, \hat{s}_{2}\right)$. Wäre dies nicht so, dann existierte ein Konsumplan $\left(\tilde{x}^{i}, \widetilde{s}_{1}^{i}, \hat{s}_{2}\right) \in x^{i}$ derart, daß $u_{i}\left(\widetilde{x}^{i}, \widetilde{s}_{1}^{i}, \hat{s}_{2}\right)>$ 
$u_{i}\left(\bar{x}^{i}, \bar{s}_{1}^{i}, \hat{s}_{2}\right)$ und $\left(\hat{p}_{x}, \hat{p}_{e}\right) \tilde{x}^{i}-\beta_{i} \hat{p}_{s}\left(\bar{s}_{1}^{i}, \hat{s}_{2}\right) \leqq I_{i}(\hat{p})$ für $i=1, \ldots, N_{1}$ erfüllt ist. Analog zur obigen Argumentation zeigt man, daß dann $\left(\tilde{x}^{i}, \tilde{s}_{1}^{i}, \hat{s}_{2}\right) \notin \hat{h}_{i}\left(\hat{p}, \hat{s}_{2}\right)$ für $i=1, \ldots, N$, womit ein widerspruch vorliegt. Wegen $\left(\bar{x}^{i}, \bar{s}_{1}^{i}, \hat{s}_{2}\right)=\widehat{h}_{i}\left(\hat{p}, \hat{s}_{2}\right)=h_{i}\left(\hat{p}, \hat{s}_{2}\right)$ für $i=1, \ldots, N_{1}$ ist auch $\bar{s}^{i}=\hat{\sigma}_{i}\left(\hat{p}, \hat{s}_{2}\right)=\sigma_{i}\left(\hat{p}, \hat{s}_{2}\right)$ für $i=1, \ldots, N_{1}$, womit median $\hat{\sigma}_{i=1, \ldots, N_{1}}\left(\hat{p}, \hat{s}_{2}\right)=$ $\operatorname{median}_{i=1, \ldots, N_{1}} \sigma_{i}\left(\hat{p}, \hat{s}_{2}\right)$. Analog zeigt man diese Behauptung $f u ̈ r ~ i=N_{1}+1$, $\ldots, N$. Deshalb gilt $\hat{\zeta}(\hat{p}, \hat{s})=\zeta(\hat{p}, \hat{s})$ und wegen $\hat{s} \in \hat{\zeta}(\hat{p}, \hat{s})$ folgt die Behauptung.

Q.E.D. 


\section{Literatur:}

Baumol, W.J. und Oates, W.E (1971), The Use of Standards and Prices for the Protection of the Environment, Swedish Journal of Econonics, 73, 42-54.

Black, D. (1958), The Theory of Committees and Elections, Cambridge University Press.

Bowen, H. (1943), "The Interpretation of Voting in the Allocation of Econonic Ressources", Quarterly Journal of Economics, 58, 27-48.

Debreu, G. (1959), Theory of Value, übersetzt als "Werttheorie", 1976, Berlin u.a..

Dudenhöffer, F. (1980), "Allokation durch Mehrheitswahl. Eine Lösung des Freifahrerproblems?", Beiträge zur angewandten Wirtschaftsforschung No. 159-80, Universität Mannheim.

Greenberg, J. (1980), "Consistent Majority Rules over Compact Sets of Alternatives",Econometrica, 47, 627-637.

Klevorick, A.K. und Kramer, G.H. (1973), "Social Choice on Pollution Management: The Genossenschaften", Journal of Public Economics, 2, 101-146.

Nikaido, H. (1968), Convex Structures and Economic Theory, Academic Press New York.

Pethig, R. (1979), Umweltökonomische Allokation mit Emissionssteuern, Tübingen.

Siebert, H. (1975), "Regional Aspects of Environmental Allocation, Zeitschrift für die gesamte Staatswissenschaft, 131 , 496-513.

Siebert, H. (1978a), Ökonomische Theorie der Umwelt, Tübingen.

Siebert, H. (1978b), "Räumliche Aspekte der Umweltallokation" in Buhr,Friedrich (Hrsg.), Konkurrenz zwischen kleinen Regionen.

Siebert, H. (1981), Economics of the Environment, Lexington, D.C. Heath.

Slutsky, S. (1977), "A Voting Model for the Allocation of Public Goods : Existence of an Equilibrium", Journal of Economic Theory, 14, 299-325.

Tietenberg, ]..H. (1974), "Derived Decision Rules for Pollution Control in a General Equilibrium Space Economy, Journal of Environmental and Management, 1, 3-16. 
Horst Siebert - 978-3-631-75607-2

Downloaded from PubFactory at 01/11/2019 03:09:22AM

via free access 
III. Regionale Umweltallokation in der zeit

Horst Siebert - 978-3-631-75607-2

Downloaded from PubFactory at 01/11/2019 03:09:22AM

via free access 
Horst Siebert - 978-3-631-75607-2

Downloaded from PubFactory at 01/11/2019 03:09:22AM

via free access 
Zur intertemporalen regionalen Umweltallokation

H. Gebauer *

1. Einleitung

1. 1 Problemstellung

"Die Umwelt als Gesamtheit der den menschlichen Lebensraum definierenden natürlichen Gegebenheiten wurde von ökonomen lange Zeit ignoriert. Luft und Wasser waren die Prototypen freier Güter - ohne Preis, nicht knapp und damit nicht Gegenstand des Wirtschaftens."1) Mitte der sechziger Jahre begann jedoch ein Umdenken, da in der industrialisierten Welt, durch die bis dahin (und jetzt zum Teil immer noch) betriebene materielle wachstumspolitik, eine Begrenzung der Schadstoffaufnahmefähigkeit des ökologischen systems fühlbar wurde. Auch die ersten ökonomischen Veröffentlichungen, die sich mit der Umweltproblematik befassen, fallen in diese zeit. 2) Danach ist ein sprunghafter Anstieg der umweltökonomischen Literatur zu verzeichnen. ${ }^{3)}$ Die Nutzung der Umwelt als Schadstoffaufnahmemedium erfolgt im allgemeinen zum "Nulltarif". Dadurch entsteht eine Diskrepanz zwischen den einzelwirtschaftlichen und den gesamtwirtschaftlichen Kosten. Diese Diskrepanz hat zur Folge, daß Fehlallokationen entstehen kön-

* Für Anregungen, kritische Anmerkungen und Hinweise möchte ich Prof. J. MoMillan, Prof. H. Siebert, F. Dudenhöffer, H. Meder, S. Toussaint und Dr. W. Vogt sowie den Mitgliedern des SFB danken.

1) Siebert, $(1979,2)$.

2) Die Klassiker Malthus, Mill und Pigou nicht mitgerechnet.

3) Einen Überblick über die Literatur der Umweltökonomie bis 1975 geben Fisher, Peterson (1976). 
nen, d.h. die Umwelt wird nicht ihrer optimalen Verwendungsmöglichkeit zugewiesen. Das Umweltproblem kann also u.a. als Allokationsproblem aufgefaßt werden. Geht man von der Existenz einer sozialen Wohlfahrtsfunktion aus, kann der statische Aspekt der Umweltallokation heute im wesentlichen als gelöst betrachtet werden, da sich Implikationen für die optimale Umweltallokation ableiten lassen.[Bonus (1972), Siebert (1975a), Tietenberg (1973)]. ${ }^{4}$ ) Auch der intertemporale Aspekt der Umweltallokation wurde schon vielfach untersucht. ${ }^{5}$ ) Durch die intertemporalen Analysen sollen auch die Interessen zukünftiger Generationen berücksichtigt werden.

Neben diesen zeitlichen Aspekten hat das Umweltallokationsproblem jedoch auch räumliche Dimensionen, da Umweltsysteme immer räumlich gebunden sind [Siebert (1978 a, 3)]. Solche räumlichen Dimensionen in einem statischen Kontext wurden z.B. behandelt von Brookshire (1978); Cumberland, Korbach (1973), Isard (1972), Siebert (1975; 1978b); verschiedene Beiträge in Siebert et al. (1979). Die räumliche Dimension in einem intertemporalen Kontext wurde bis jetzt nur von wenigen Autoren untersucht. ${ }^{6}$ )

Im folgenden werden zwei Teilaspekte dieser räumlichen Dimension des Umweltproblems untersucht. Dazu werden im restlichen Teil der Einleitung einige zentrale Begriffe geklärt. Im zweiten Teil schließt sich ein Uberblick über die Ergebnisse der Modellanalyse an. In Teil 3 wird die Modellökonomie beschrieben und in den verbleibenden Teilen 4 und 5 werden anhand dieser ökonomie die Wohlfahrtseffekte einseitiger bzw. zweiseitiger interregionaler Verschmutzung im zeitablauf diskutiert.

\footnotetext{
4) Vgl. Siebert $(1979,8)$.

5) Vgl. z.B. D'Arge, Kogiku (1973), Comolly (1975), Converse (1974), Fisher et al. (1972), Forster (1973 und 1977 b), Keeler et al. (1972), Mäler (1974), Vogt (1981), Wright (1974).

6) Vgl. Førsund (1979), Nijkamp (1978), sowie die Raum-Zeit-Modelle von Isard, Liossatos (1979), die sich allerdings nicht speziell mit der Umweltproblematik befassen.
} 
1.2 Die Umwel.t als Schadstoffaufnahmemedium ${ }^{7)}$

Bei Produktion und Konsum von Gütern fallen Kuppelprodukte an, die weder für: den Konsum noch die Produktion verwertbar sind, d.h. sie werden an die Umwelt abgegeben. Dabei kann man solche Kuppelprodukte unterscheiden, die keinen Einfluß auf die Umweltqualität haben, während andere die Umwelt als Schadstoffe belasten. Diese schädlichen Abfallstoffe werden im folgenden Emissionen genannt. Diese Emissionen gelangen ins ökosystem,8) dabei unterliegen sie diffusiven und transformativen Einflüssen, deren Resultat die Umweltbelastung in Gestalt der Menge an schädlichen Stoffen ist, die sich zu einem Zeitpunkt in einem bestimmten Umweltmedium 9l befinden. Dieser Bestand an Schadstoffen wird im folgenden Imnission genannt.

Das ökosystem hat die Eigenschaft, sich teilweise selbst zu reinigen. "Als Selbstreinigung (Assimilation)... lassen sich alle chemischen und biologischen prozesse im ökosystem auffassen, die zur Beseitigung einer Belastung führen."[ Umweltgutachten (1978, 25)]. Das Ausmaß der Assimilationsfähigkeit bestimmt also, wie Emissionen eines bestimmten Zeitpunktes sich in späteren Zeitpunkten auswirken, indem sie den Immissionsbestand vergrößern, der seinerseits durch Assimilation verringert wird. Liegt Gleichheit von assimilationsbedingter Immissionsabnahme und emissionsbedingter Immissionszunahme vor, hat das ökosystem einen statio nären Zustarid erreicht.

Die Komplexität der eben beschriebenen ökologischen Zusammenhänge erfordert. Vereinfachungen, um die ökologisch-ökonomischen Interdependenzien darstellen zu können. In den Modellen im 3. und 4. Teil werden folgende Vereinfachungen vorgenommen:

- es wird nur ein Schadstoff betrachtet, der bei der Produktion anfällt, d.h. die Emissionen der Regionen sind homogen,

7) Vgl. Vogt (1981, 11-18).

8) Unter ökosystem versteht man dabei "funktionelle Einheiten aus Organismen ("Lebensgemeinschaft" aus Pflanzen und Tieren, Biozönose) und unbelebter Umwelt ("Lebensraum", Biotop) ... Die verschiedenen ökosysteme des Landes und der Gewässer ordnen sich zur ökosphäre."[Umweltgutachten (1978, 20)].

9) Land, Wasser, Luft 
- zwischen den einzelnen Umweltmedien wird nicht differenziert,

- die Immissionen sind homogene, lokale öffentliche Güter,

- über ökologische Prozesse (Diffusion und Transformation) liegt vollständige Information vor,

- nur durch die Produktion kommt es zu Emissionen.

\subsection{Einige Anmerkungen zur Wohlfahrtsfunktion}

Durch die zusätzlichen Aspekte der zeitlichen Nutzung der Umwelt als Schadstoffrezeptor wird das Allokationsproblem ausgeweitet, denn es wird nicht nur die Immission der Gegenwart, sondern auch die der zukunft berücksichtigt. Die die Immission bestimmende Pigou-Steuer, der Emissionsschattenpreis, spiegelt demnach gegenwärtige und zukünftige Externalitäten wider. In der Analyse wird angenommen, daß die steuern von zentralen planern gesetzt werden, die die Präferenzen der Bewohner genau kennen, bzw. über einen nicht näher spezifizierten - institutionell verankerten Abstimmungsproze $\beta$ erfragt haben, welche in Gestalt von Nutzenfunktionen. formalisierbar sind. ${ }^{10)}$ Diesen sozialen Wohlfahrtsfunktionen liegen vereinfachende Annahmen zugrunde: ${ }^{11)}$

(i) die Planungsbehörden unterstellen, daß sich die Präferenzen im Zeitablauf nicht ändern, d.h. die gegebenen Wohlfahrtsfunktionen sind zeitlich invariant,

(ii) die gesellschaftlichen Nutzenniveaus sind zeitlich unabhängig,

(iii) die intertemporale Wohlfahrt wird durch Addition der zeitpunktbezogenen gesellschaftlichen Nutzen ermittelt, was Kardinalität der Wohlfahrtsfunktionen impliziert.

Bei der Wahl der gesellschaftlichen Diskontrate, die die Gegenwartspräferenz der Gesellschaft zum Ausdruck bringt [ Siebert (1978, 150)], entstehen ebenfalls Aggregationsprobleme, die nicht gelöst sind. Wird eine hohe Diskontrate gewählt, dann wird ein hohes Gewicht auf die Präferenzen der gegenwärtigen Generation gelegt, während bei einer niedrigen zeitpräferenzrate die Inte-

10) Vgl. Vogt (1981, 21), Mäler (1974, 104-105) .

11) Vogt $(1981,21)$. 
ressen zukünftiger Generationen stärker gewichtet werden. Da die Wahl der Diskontrate für den Planungszeitraum von derjenigen Generation vorgenommen wird, die im Anfangszeitpunkt lebt und "Zukunftsgenerationen keine Mitgestaltungsmöglichkeiten besitzen", kann sie "als ein von der Gegenwartsgeneration zu treffendes Werturteil begriffen werden". [ $\operatorname{Vogt}(1981,22)] .12)$ Im folgenden wird von einer positiven, konstanten Diskontrate ausgegangen.

\subsection{Dezentrale Entscheidungen}

Die intertemporale Umweltallokation wurde bisher hauptsächlich "in a wonderland of no other decision units" [ Nijkamp (1978, 154)] untersucht, ${ }^{13}$ ) d.h. spill-over Effekte wurden per Definition ausgeschlossen. E؛s wird also davon ausgegangen, daß es nur einen Entscheidungsbefugten oder zumindest nur eine Zielfunktion gibt [ (Kydland $(1975,321)]$. Jedoch werden gerade bei der Umweltverschmutzung Menschen betroffen, die weder an den die Verschmutzung erzeugenden Aktivitäten beteiligt sind noch davon profitieren. Beispiele hierfür sind u.a. die Unterlieger-Oberlieger-Problematik sowie die "Politik des hohen Schornsteins". Bei ersterer hat man es mit der. Problem zu tun, daß Regionen, die an einem Flußlauf relativ nahe an der Quelle liegen, diesen FluB ohne Rücksicht auf Regionen verschmutzen, die näher an der Mündung liegen, ohne daß die Unterlieger etwas von der die Verschmutzung verursachenden Produktion $z u$ haben brauchen. Bei der Politik des hohen Schornsteins geben Unternehmen ihre Schadstoffe, mit Billigung der Regicnalbehörden, so an die Umwelt ab, daß nur ein Teil in der eigener. Region ins ökosystem gelangt, während der andere Teil die ökologischen systeme anderer Regionen belastet.

In der im 4. und 5. Teil vorgenommenen Modellanalyse werden die oben angeführten Probleme betrachtet. Während im 4 . Teil bei der Unterlieger-oberlieger-Problematik noch ein kontrolltehoretisches Modell benutz

12) Vgl. zur Problematik der Diskontrate u.a. auch Krutilla, Fisher (1975, 60-69), Herfindahl, Kneese (1974), Page (1977).

13) Vgl. Fußnote 5 . 
theorie angewandt werden, da die untersuchten Regionen gegenseitig voneinander abhängig sind. Bei beiden Modellvarianten ergibt sich das Problem, welche Informationen den Regionalregierungen zur Verfügung stehen. Wir wollen im folgenden davon ausgehen, daß die "importierten" Emissionen von den jeweiligen Regionalbehörden als gegeben betrachtet werden [ Vgl. Bernstein (1977)] .

\section{Fragestellungen und Uberblick}

Im folgenden wird von einer Volkswirtschaft ausgegangen, die in zwei Regionen aufgeteilt ist. Wir nehmen an, daß die Wohlfahrt der Regionen durch den jeweiligen Güterberg und den jeweiligen Schadstoffbestand determiniert wird. Weiterhin wird unterstellt, daß die Regionalregierungen nur in Eigeninteresse handeln, d.h. die regionenspezifische Wohlfahrt maximieren ohne Rücksicht auf die andere Region. Solch eine ökonomie kann als klassich-föderalistisch [Frey (1979)] bezeichnet werden, d.h. die Regionalbehörden haben das Recht, Zielkonflikte aufgrund der Präferenzen ihrer eigenen Bevölkerung zu lösen.

In der in Abschnitt 4 vorgenommenen intertemporalen Modellanalyse wird angenommen, daß eine Region (Region 2) durch Diffusionsvorgänge das Ökosystem der anderen Region (Region 1) beeinflußt. Es wird gezeigt, daß Region 1, d.h. die durch Emissionen der Region 2 mitverschmutzte Region, ihr Wohlfahrtskalkül nach der Umweltpolitik der Region 2 ausrichten muß. ${ }^{14)}$ Wird in Region 2 eine relativ unwirksame, bzw. keine Umweltpolitik betrieben, sind die Emissionen in Region 2 hoch und damit auch der Anteil, der nach Region 1 gelangt. Für eine intertemporal wohlfahrtsoptimale Politik muß die Regionalregierung der Region 1 eine hohe Steuer setzen, so daß die Emissionen in Region 1 niedrig sind. Dies bedeutet aber, da $B$ auch die Güterproduktion in Region 1 relativ niedrig ist, während die verschmutzung, trotz der niedrigen Emissionen, hoch ist. Region 1 erleidet also durch

14) Die Struktur der ökonomie ist in Schaubild 1 dargestellt, wobei der gestrichelte Pfeil erst für die ökonomie im 4. Teil gilt. 


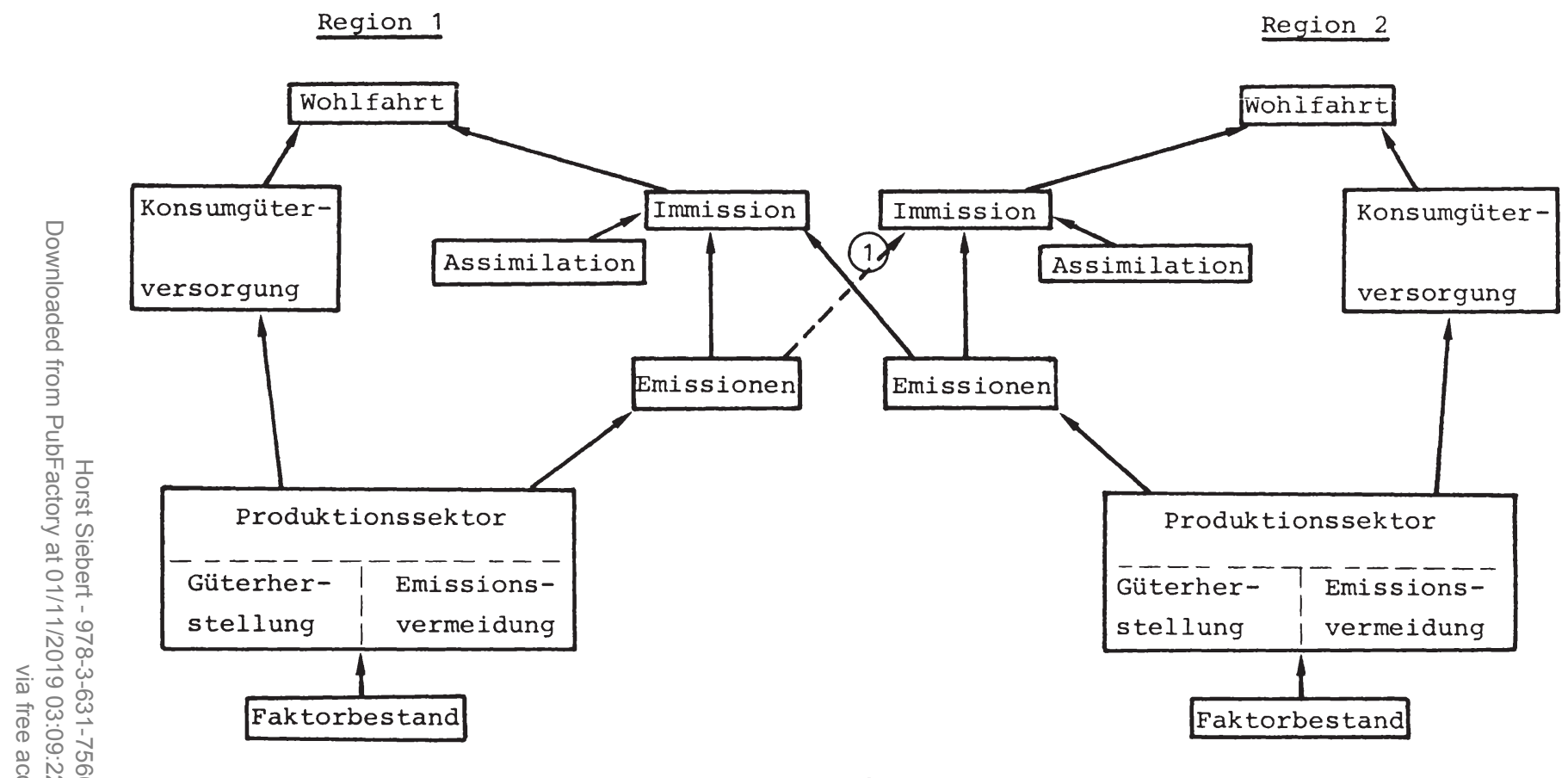

Schaubild 1: Struktur der ökonomie 
die "laissez-faire" Politik in Region 2 Wohlfahrtsverluste durch eine relativ niedrige Güterversorgung und eine relativ schlechte Umweltqualität.

Geht man von dem anderen Extrem aus, daß in Region 2 die Emissionen Null sind, d.h. dort wird eine sehr stringente Umweltpolitik betrieben, ist in Region 1 eine bessere Umweltqualität, höhere Emissionen und damit eine größere Güterproduktion möglich, als wenn in Region 2 Emissionen anfallen, so daß in Region 1 Wohlfahrtsgewinne durch die Umweltpolitik der Region $2 \mathrm{zu}$ verzeichnen sind. Aus diesen beiden, nicht explizit im Modell analysierten, Extremen, sieht man die einseitige Abhängigkeit der Region 1 von Region 2, wenn die Regionalregierungen nur das Eigentinteresse ihrer Regionen im Auge haben.

Im 5. Teil wird dann nicht mehr nur von einer einseitigen Abhängigkeit ausgegangen, sondern jede der beiden Regionen "exportiert" einen Teil ihrer Emissionen in die andere Region (vgl. Schaubild 1). Es wird gezeigt, daß ein Gesamtgleichgewicht existiert, in dem alle Werte stationär sind, wenn sich die Regionalbehörden so verhalten, daß sich die Emissionen zu einer steadyState Reaktionsfunktion hin bewegen.

\section{Die Modellökonomie}

Wir betrachten eine Modellökonomie, die in zwei Regionen aufgeteilt ist. Die beiden Regionen sollen derart miteinander verbunden sein, daß Region i Region j durch Emissionen mitver- schmutzt und umgekehrt. ${ }^{15)}$ Der Modellrahmen wird von den üblichen Modellen für eine Ein-Region-ökonomie übernommen [ (vgl. z.B. D'Arge, Kogiku (1973), Forster (1977), Vogt (1981)]. Mit leichten Modifikationen werden diese Modelle dahingehend erweitert, daß sie auf eine Zwei-Regionen-ökonomie angewandt werden können.

15) Die einseitige Verschmutzung wird in Teil 4 untersucht, und die zweiseitige in Teil 5. 


\subsection{Produktionstechnologie}

Die Produktionstechnologie der Region $i$ wird folgendermaßen spezifiziert: ${ }^{16)}$

Bei der Herstellung des Gutes $x_{i}$ durch den Einsatz des Faktors $R_{i}$ fällt eine umweltschädigende Kuppelproduktmenge $E_{i}$ an, die weder für den Konsum noch für die produktion verwertbar ist und die emittiert wird und damit die Umwelt belastet. Diese Produktionstechnik wird durch die streng konkave, zweifach stetig differenzierbare Produktionsfunktion dargestellt:

$$
\left.x_{i} \leq f^{i}\left(R_{i}, E_{i}\right)^{17}\right)
$$

mit dem konvexen Difinitionsbereich

$$
D\left(f^{i}\right)=\left\{\left(R_{i}, E_{i}\right) \mid\left(R_{i}, E_{i}\right) \in R_{+}^{2} \wedge R_{i} \geq g_{i} E_{i}, g_{i}>0\right.
$$
und konstant \}

und den Eigenschaften

$$
f^{i}\left(O, E_{i}\right)=0, f^{i}\left(R_{i}, 0\right) \geq 0 \forall R_{i}>0 f_{R}^{i}>0, f_{E}^{i}>0
$$

Dieser sogenannte "Nettoansatz" ${ }^{18)}$ betrachtet die Emissionen $E_{i}$ formal als zusätzlichen Produktionsfaktor, der die Inanspruchnahme der Umwelt als Schadstoffaufnahmemedium durch den Produktionsprozeß ausdrückt. Es werden nur diejenigen Emissionen betrachtet, die tatsächlich an die Umwelt abgegeben werden, d.h. sektorinterne Entsorgungsaktivitäten werden durch diese produktionstechnologie implizit berücksichtigt.

Die Bedingung

$$
R_{i} \geq g_{i} E_{i}
$$

ordnet alternativen Faktoreinsätzen die höchstmögliche Kuppelproduktmenge $z u$, sie drückt also die Kuppelproduktionskapazität

16) Eine allgemeinere Form dieser Produktionstechnologie wird in Pethig (1975, 100-106) entwickelt.

17) Die Variablen, die durchweg Funktionen der Zeit sind, werden aus Vereinfachungsgründen nicht explizit als zeitabhängig ausgewiesen, wenn sich dadurch keine Fehldeutungen ergeben.

18) Vgl. Siebert et al. (1980, 29-42). 
aus. In Schaubild 2 wird der Produktionsraum der Region i dargestellt:

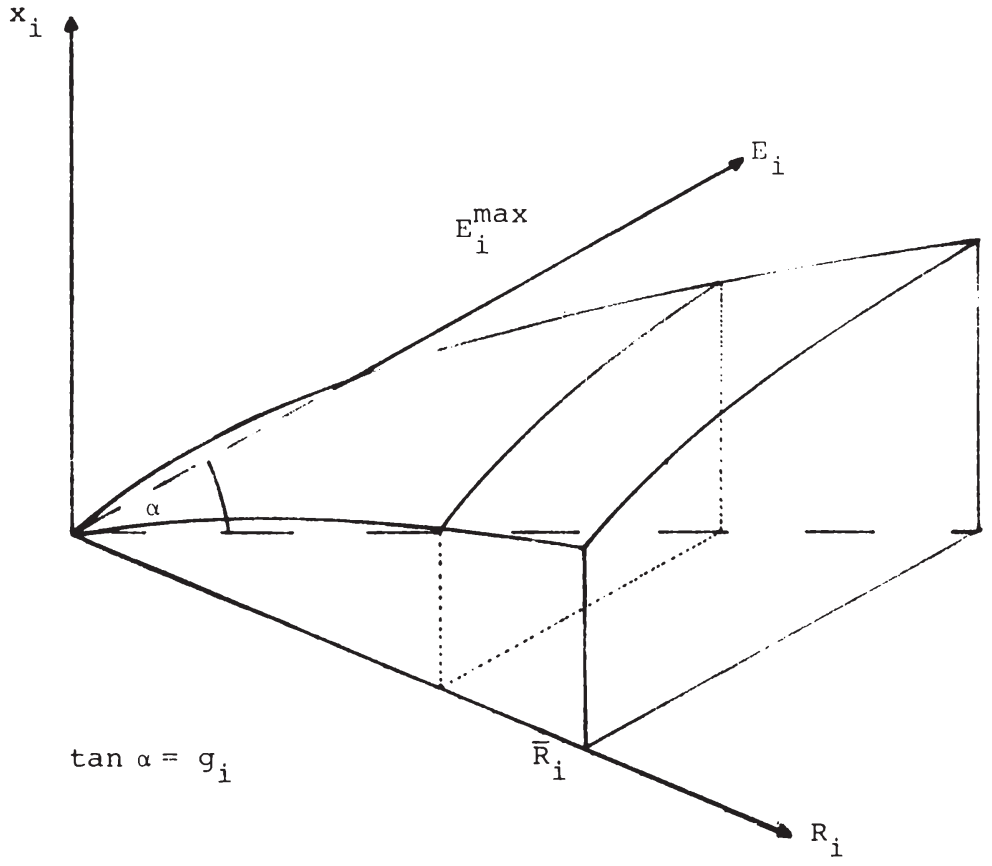

Schaubild 2: Produktionsraum der Region i.

Die Faktorrestriktion ist

$$
\bar{R}_{i}-R_{i} \geq 0
$$

wobei $\bar{R}_{i}$ für alle $t$ konstant und gleich ist, d.h. interregionale Faktormobilität ist ausgeschlossen.

\section{2 ökologisches System}

Die zeitliche Immissionsänderung $\dot{S}_{i}$ in Region $i$ ist abhängig von den Emissionen und der Assimilationsfähigkeit in Region i und dem Teil der Emissionen von Region $j$, der nach Region $i$ "exportiert" wird: 


$$
\dot{S}_{i}=\left(1-\gamma_{i}\right) E_{i}+\gamma_{j} E_{j}-\alpha_{i} S_{i} \quad i, j=1,2 \quad i \neq j
$$

mit $\alpha_{i} \in[0,1]$ und konstant, dem Assimilationskoeffizient der Region $i$, und $\gamma_{i} \in\left[0, \frac{1}{2}\right)$ und konstant, dem Anteil der Emissionen der Region $i$, der durch Diffusionsvorgänge das ökosystem der Region $j$ beeinflußt. Aus den Gleichungen (4) lassen sich stationäre ökologische Zustandsfunktionen herleiten, mit deren Hilfe die Menge der Emission-Immission-Kombination hinsichtlich ihrer ökologischen Eigenschaften unterteilt werden kann. Unter einem stationären Zustanã des ökosystems versteht man die Gleichheit von assimilationsbedingter Immissionsabnahme und emissionsbedingter Immissionszunahme. ${ }^{19)}$

Aus (4) erhält man

$$
S_{i}=\frac{1}{\alpha_{i}}\left[\left(1-\gamma_{i}\right) E_{i}+\gamma_{j} E_{j}\right]
$$

In Schaubild 3 ist die stationäre ökologische Zustandsfunktion für Region $i$ be $i$ alternativen $E_{j}$-Werten dargestellt:

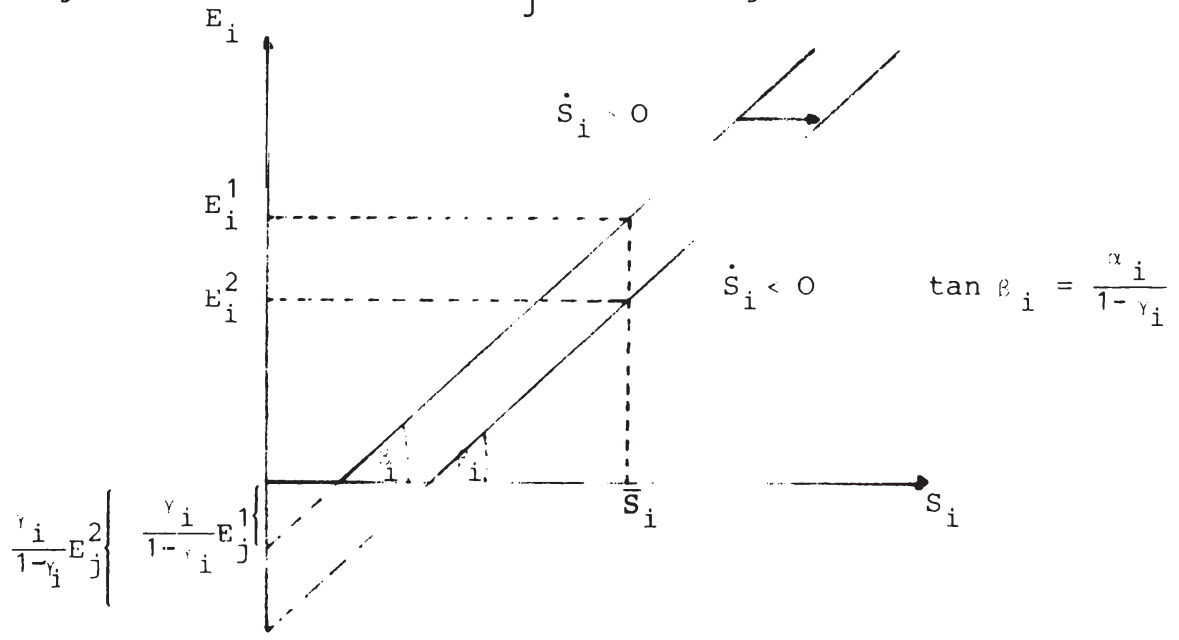

Schaubild 3: Stationäre ökologische Zustandsfunktion der Region i.

19) Vogt (1981),14) spricht in diesem Zusammenhang von ökologischem Gleichgewicht. 
Aus Schaubild 3 sieht man, daß der stationäre Zustand des ökosystems der Region $i$ von den Emissionen der Region $j$ mitabhängt. Je größer die Emissionen der Region $j$ sind, desto weiter nach rechts verschiebt sich die stationäre ökologische Zustandsfunktion der Region $i, d . h$. je größer $E_{j}$ ist, desto kleiner muß $E_{i}$ sein, um einen bestimmten stationären ökologischen Zustand $\bar{S}_{i} z u$ halten. Die Steigung dieser Funktion ist sowohl von der Assimilationsfähigkeit des ökosystems der Region als auch von dem Diffusionskoeffizienten abhängig. Je höher der Diffusionskoeffizient $\gamma_{i}$, d.h. je mehr Emissionen $E_{i}$ nach Region $j$ gelangen, desto steiler ist die stationäre ökologische Zustandsfunktion. Oberhalb der Funktion nehmen die Immissionen $z u\left(\dot{S}_{i}>0\right)$, während sie unterhalb abnehmen $\left(\dot{S}_{i}<0\right)$.

Im folgenden wollen wir davon ausgehen, daß die Assimilationskapazität der Region $i\left(\alpha_{i} S_{i}\right)$ bei eigener Nullemission $\left(E_{i}=0\right)$ dazu ausreicht, die maximal möglichen Emissionsimporte aus Region $j\left(\gamma_{j} E_{j}^{\max }\right)$ abzubauen. Es soll also gelten:

$$
\left.\alpha_{i} S_{i}\right|_{E_{i}=0}>\gamma_{j} E_{j}^{\max } \text {. }
$$

\subsection{Die Wohlfahrtsfunktionen}

Die Wohlfahrt der Region i sei vom Güterberg und der Verschmutzung der Region $i$ im Zeitpunkt $t$ abhängig:

$$
u_{i}=u^{i}\left(x_{i}, s_{i}\right)
$$

Diese Funktion sei mehrfach stetig differenzierbar, konkav und separabel und besitze außerdem folgende Eigenschaften: $u_{x}^{i}\left(0, s_{i}\right)=\infty, u_{x}^{i}>0 \forall x_{i}>0, u_{s}^{i}<0, u^{i}(0,0)=0$. Durch die Wohlfahrtsfunktionen wird die Bewertung der Umweltbehörden ausgedrückt, die sie unterschiedlichen Güteroutputs und Schadstoffbeständen beimessen. 


\section{Einseitige Verschmutzung}

Wir betrachten den Fall, daß Region 2 Region 1 mitverschmutzt, während Region 1 keinen Einfluß auf Region 2 nimmt, d.h. $\gamma_{1}=0$ und $\gamma_{2}>0$. Die Gleichungen (4) können dann geschrieben werden:

(4.1) $\quad \dot{S}_{1}=E_{1}+\gamma_{2} E_{2}-\alpha_{1} S_{1}$

(4.2) $\quad \dot{\mathrm{S}}_{2}=\left(1-\gamma_{2}\right) \mathrm{E}_{2}-\alpha_{2} \mathrm{~S}_{2}$

und die ökologischen stationaritätsbedingungen sind

$$
\begin{aligned}
& (5.1) \quad S_{1}=\frac{1}{\alpha}-\left[E_{1}+\gamma_{2} E_{2}\right] \\
& (5.2) \quad S_{2}=\frac{1-\gamma_{2}}{\alpha_{2}} E_{2}
\end{aligned}
$$

4.1 Die Optimierungsprobleme und die Optimalbedingungen

Die Umweltbehörden der Regionen sehen sich also folgenden intertemporalen Optimierungsproblemen gegenüber $(i=1,2)$ :

$\operatorname{Max}_{R_{i}} W^{i}={ }_{0}^{\infty} u^{i}\left(x_{i}, s_{i}\right) e^{-\zeta^{t}} d t$

u.d.B.

$$
\begin{aligned}
& \text { (4.1) } \dot{S}_{1}=E_{1}+\gamma_{2} E_{2}-\alpha_{1} S_{1} \\
& \text { (4.2) } \quad \dot{S}_{2}=\left(1-\gamma_{2}\right) E_{2}-\alpha_{2} S_{2}
\end{aligned}
$$$$
x_{i}, R_{i}, E_{i}, s_{i} \geq 0 \quad \forall t \in[0, \infty)
$$$$
s_{i}(0)=s_{i}^{O} \text { gegeben }
$$

$\zeta$ gegeben und positiv.

Auf diese Kontrollprobleme -läßt sich Pontryagins Maximumprinzip anwenden. 20) Die Lagrange-Funktionen in laufenden Werten sind:

20) Vgl. Takayama (1974, 648-650, Theorem 8.C.1 und Korollar). Daraus ergibt sich, daB die abzuleitenden Bedingungen notwendig und hinreichend für ein Optimum sind. 
$L^{1}\left(x_{1}, R_{1}, E_{1}, S_{1}, P_{1}, \lambda_{1}^{1}, \lambda_{2}^{1}, \lambda_{3}^{1}\right)=$

(8)

$$
\begin{aligned}
& u^{1}\left(x_{1}, S_{1}\right)+p_{1}\left[E_{1}+r_{2} E_{2}-\alpha_{1} S_{1}\right]+ \\
& +\lambda_{1}^{1}\left[f^{1}\left(R_{1}, E_{1}\right)-x_{1}\right]+\lambda_{2}^{1}\left[R_{1}-g_{1} E_{1}\right]+\lambda_{3}^{1}\left[\bar{R}_{1}-R_{1}\right] .
\end{aligned}
$$

$L^{2}\left(x_{2}, R_{2}, E_{2}, S_{2}, P_{2}, \lambda{ }_{1}^{2}, \lambda 2, \lambda \frac{2}{2}\right)=$

$$
\begin{aligned}
& u^{2}\left(x_{2}, s_{2}\right)+p_{2}\left[\left(1-\gamma_{2}\right) E_{2}-\alpha_{2} S_{2}\right]+ \\
& +\lambda_{1}^{2}\left[f^{2}\left(R_{2}, E_{2}\right)-x_{2}\right]+\lambda_{2}^{2}\left[R_{2}-g_{2} E_{2}\right]+\lambda_{3}^{2}\left[\bar{R}_{2}-R_{2}\right],
\end{aligned}
$$

woraus sich folgende Bedingungen ergeben $(i=1,2)$ :

(10)

$$
\begin{aligned}
& L_{x}^{i}=u_{x}^{i}-\lambda_{1}^{i} \leq 0 \quad x_{i} L_{x}^{i}=0 \\
& \mathrm{~L}_{\mathrm{R}}^{i}=\lambda_{1}^{i} \mathrm{f}_{\mathrm{R}}^{i}+\lambda_{2}^{i}-\lambda \frac{i}{3} \leq \mathrm{O} \quad \mathrm{R}_{\mathrm{i}} \mathrm{L}_{\mathrm{R}}^{i}=0 \\
& \mathrm{~L}_{\mathrm{E}}^{1}=\mathrm{p}_{1}+\lambda_{1}^{1} \mathrm{f}_{\mathrm{E}}^{1}-\lambda_{2}^{1} \mathrm{~g}_{1} \leq \mathrm{O} \mathrm{E}_{1} \mathrm{~L}_{\mathrm{E}}^{1}=\mathrm{O} \\
& L_{E}^{2}=\left(1-\gamma_{2}\right) p_{2}+\lambda_{1}^{2} f_{E}^{2}-\lambda_{2}^{2} g_{2} \leq O E_{2} L_{E}^{2}=0 \\
& L_{p}^{1}=\dot{s}_{1}=E_{1}+r_{2} E_{2}-a_{1} s_{1} \\
& L_{p}^{2}=\dot{s}_{2}=\left(1-\gamma_{2}\right) E_{2}-\alpha_{2} S_{2} \\
& L_{S}^{i}=-\dot{p}_{i}+\zeta p_{i}=u_{S}^{i}-\alpha_{i} p_{i} \\
& \dot{p}_{i}=\left(\zeta+\alpha_{i}\right) p_{i}-u_{S}^{i} \\
& \lim _{t \rightarrow \infty} e^{-\zeta t} p_{i}(t)=0 \\
& \lambda i_{1}^{i}\left[f^{i}\left(R_{i}, E_{i}\right)-x_{i}\right]=0 \quad \lambda_{1}^{i} \geq 0 \\
& \lambda \frac{i}{2}\left[R_{i}-g_{i} E_{i}\right]=0 \quad \lambda_{2}^{i} \geq 0 \\
& \lambda_{3}^{i}\left[\bar{R}_{i}-R_{i}\right]=0 \quad \lambda_{3}^{i} \geq 0
\end{aligned}
$$

\subsection{Die Eigenschaften der wohlfahrtsoptimalen Entwicklung} der Regionen 1 und 2

Aus den Wohlfahrtsfunktionen $u^{i}$ folgt, daß im Optimum positiver Güterkonsum vorliegt, $d . h$. es gilt $x_{i}>0$. 
Daraus folgt:

(I) $u_{x}^{i}=\lambda_{1}^{i}>0$

(II) $R_{i}>0$ und deshalb $\lambda_{1}^{i} f_{R}^{i}+\lambda{ }_{2}^{i}=\lambda_{3}^{i}>0$, was vollbeschäftigung impliziert, d.h. $R_{i}=\bar{R}_{i}$.

Sei $E_{i} \in\left(0, \frac{1}{g}-\bar{R}_{i}\right)$. Daraus folgt, daß $\lambda_{2}^{i}=0$ ist und deshalb $\lambda_{3}^{i}=\lambda i_{1}^{i} f_{\mathrm{R}}^{i}$. D. ${ }^{\mathrm{h}}$., daß im Optimum der Schattenpreis des Gutes $x_{i}\left(\lambda \frac{i}{1}\right)$ seinen Grenzkosten entsprechen muß $\left.\left(\lambda_{3}^{i} \mid f_{R}^{i}\right) .21\right)$ weiter folgt, da $E_{i}=0$ ist

(11) $p_{1}+\lambda_{1}^{1} f_{E}^{1}=0 \quad b z w$.

(12) $\left(1-\gamma_{2}\right) p_{2}+\lambda_{1}^{2} f_{E}^{2}=o^{22)}$ woraus man die Bedingungen

(11') $p_{1}+u_{x}^{1} f_{E}^{1}=0 \quad b z w$.

(12') $\left(1-r_{2}\right) p_{2}+u_{x}^{2} f_{E}^{2}=0$ erhält.

Aus (11') bzw. (12') können Funktionen $\hat{E}_{1}\left(p_{1}\right)$ und $\hat{E}_{2}\left(p_{2}\right)$ abgeleitet werden, die alternativen werten von $p_{1} b z w . p_{2}$ die jeweiligen optimalen Emissionsniveaus zuordnen.

$$
\begin{aligned}
& E_{1}=\hat{E}_{1}\left(p_{1}\right): \begin{cases}E_{1}=0 & \forall p_{1} \leq p_{1}^{\prime}, p_{1}^{\prime} \equiv-u_{x}^{1} f_{E}^{1} \mid E_{1}=0 \\
E_{1}>0 & \left.\hat{E}_{1 p^{\prime}}>0^{23}\right)_{\forall p_{1}} \in\left(p_{1}^{\prime}, p_{1}^{\prime}\right), p_{1}^{\prime} "_{1} \equiv-u_{x}^{1} f_{E}^{1} \mid E_{1}=E_{1}^{\max } \\
E_{1}=E_{1}^{\max } & \forall p_{1} \geq p_{1}^{\prime \prime}\end{cases} \\
& E_{2}=\hat{E}_{2}\left(p_{2}\right): \begin{cases}E_{2}=0 & \forall p_{2} \leq p_{2}^{\prime}, p_{2}^{\prime} \equiv-\left.\frac{u_{x}^{2} f_{E}^{2}}{1-r 2}\right|_{E 2}=0 u_{x}^{2} f_{E}^{2} \\
E_{2}>0, & \hat{E}_{2 p^{2}}>0 \quad \forall p_{2} \epsilon\left(p_{2}^{\prime}, p_{2}^{\prime \prime}\right), p_{2}^{\prime \prime} \equiv-\frac{x}{1-r_{2}} \mid E_{2}=E_{2}^{\max } \\
E_{2}=E_{2}^{\max } & \forall p_{2} \geq p_{2}^{\prime \prime}\end{cases}
\end{aligned}
$$

21) Vgl. zu dieser Interpretation in statischem Kontext Siebert (1978a, 40).

22) D.h. die Bewertung des Zukunftsschadens $p_{1}$ bzw. $\left(1-\gamma_{2}\right) p_{2}$ muß dęm Wertgrenzprodukt bezüglich der Emissionen entsprechen.

23) $d E$

$$
\frac{d E_{1}}{d p_{1}}=-\frac{1}{u_{X X}^{T}\left(f_{E}^{1}\right)^{2}+u_{X E E}^{T} f \frac{T}{T E}}>0
$$


Die Emissionen $E_{1}\left(E_{2}\right)$ sind c.p. umso höher, je niedriger der gesamte Zukunftsschaden der laufenden Umweltverschmutzung bewertet wird, d.h. je kleiner der Betrag von $p_{1}\left(p_{2}\right)$ ist. Setzt man (13) bzw. (14) in (10.IV.1) und (10.IV.2) ein und berücksichtigt (V), erhält man das Differentialgleichungssystem (EDGS) :

$$
\begin{aligned}
& \dot{s}_{1}=\hat{E}_{1}\left(p_{1}\right)+\gamma_{2} \hat{E}_{2}\left(p_{2}\right)-\alpha_{1} s_{1} \\
& \dot{p}_{1}=\left(\zeta+\alpha_{1}\right) p_{1}-u_{s}^{1} \\
& \dot{s}_{2}=\left(1-\gamma_{2}\right) \hat{E}_{2}\left(p_{2}\right)-\alpha_{2} s_{2} \\
& \dot{p}_{2}=\left(\zeta+\alpha_{2}\right) p_{2}-u_{s}^{2}
\end{aligned}
$$

Dieses DGS läßt sich in zwei Teile aufspalten: Da (15.III) und (15.IV) unabhängig von (15.I) und (15.II) sind, kann man dieses Untersystem, das für Region 2 gilt, für einen gegebenen Anfangspunkt $\left(\mathrm{s}_{2}^{\mathrm{O}}, \mathrm{p}_{2}(\mathrm{O})\right)$ lösen, da die Argumente der Funktionen $\dot{\mathrm{S}}_{2}, \dot{\mathrm{p}}_{2}$ stetig sind und (zumindest intervallweise) stetige erste Ableitungen haben. 24) Das Untersystem für Region 2 weist ein eindeutiges, stationäres Gleichgewicht $\left(\mathrm{S}_{2}^{\infty}, \mathrm{p}_{2}^{\infty}\right)$ auf, ${ }^{25)}$ das einen stattelpunkt $\left.{ }^{26}\right)$ darstellt. Man erhält dann für $\mathrm{E}_{2}=\hat{\mathrm{E}}_{2}\left(\mathrm{p}_{2}^{\infty}\right)$ einen eindeutigen Wert, der in (15.I) eingesetzt wird, so daß auch das DGS für Region 1 ein eindeutiges, stationäres Gleichgewicht $\left(\mathrm{S}_{1}^{\infty}, \mathrm{p}_{1}^{\infty}\right)$ besitzt. Jedoch ist diese Lösung nicht unabhängig von der Lösung in Region 2, so daB die Bewegungseigenschaften des DGS für Region 1 mit von den Bewegungseigenschaften des DGS für Region 2 abhängen .

Schaubild 4 zeigt im IV. Quadranten das $\left(p_{2}, s_{2}\right)$ Phasendiagramm, das die Bewegungseigenschaften des DGS (15.III) und (15.IV), also für Region 2 darstellt. Die $\dot{S}_{2}=0-$ Kurve wird mit Hilfe der Graphen der Gleichungen (5.2) und (14) abgeleitet.

24) Vgl. Hadley/Kemp (1973, 370, Theorem A III-1).

25) Die Hauptminoren der Jacobischen Matrix des Systems haben im Gleichgewicht $\left(S_{2}^{\infty}, p_{2}^{\infty}\right)$ das gleiche negative Vorzeichen (Theorem 7 in Gale, Nikaido $(1965,91)$.

26) Die Determinante der Jacobischen Matrix ist negativ, Forster $(1977 a, 43)$. 
Es gilt

$$
S_{2}=\frac{1-\gamma_{2}}{\alpha_{2}} \hat{E}_{2}\left(p_{2}\right)^{27)}
$$

Für alle Punkte, die oberhalb (unterhalb) des Graphen von (16) liegen, gilt $\dot{\mathrm{S}}_{2}>0\left(\dot{\mathrm{S}}_{2}<0\right)$. Die $\dot{\mathrm{p}}_{2}=0$-Kurve ist folgendermaßen definiert:

$$
\left.p_{2}=\frac{u_{s}^{2}}{\left(+\alpha_{2}\right.} 28\right)
$$

a.h. es gilt $\dot{p}_{2}>0\left(\dot{p}_{2}<0\right)$, oberhalb (unterhalb) des Graphen von (17). Der steady state-Punkt ist der schnittpunkt der $\dot{S}_{2}=0$-Kurve mit der $\dot{\mathrm{p}}_{2}=\mathrm{O}-\mathrm{Kurve}$, so daß der IV. Quadrant in 4 sektoren mit unterschiedlichen Bewegungsrichtungen eingeteilt wird.

In Schaubild 4 sind nur die stabilen Aste der Trajektorien eingezeichnet, die für $\mathrm{T}=\infty$, mit der optimaltrajektorie übereinstimmen, je nachdem, ob die Anfangsverschmutzung kleiner oder größer als di.e stationäre Verschmutzung ist, obwohl es unendlich viele Trajektorien gibt, die jedoch instabil sind. ${ }^{29}$ Der optimale Anfangswert des Immissionsschattenpreises der Region $2 \mathrm{p}_{2}$ ist u.a. abhängig von der Anfangsverschmutzung in dieser Region. $\mathrm{p}_{2}$ (O) muß c.p. umso höher (niedriger) sein, je niedriger (höher) die Anfangsverschmutzung ist.

Ein analoges Schaubild wird nun für Region 1 abgeleitet. Die $\dot{\mathrm{p}}_{1}=\mathrm{O}$-Kurve ist genauso abzuleiten wie die $\dot{\mathrm{p}}_{2}=0$-Kurve:

27) Die steigung der $\dot{S}_{2}=0$-Kurve ist positiv: $\frac{d S_{2}}{d p_{2}}=\frac{1-\gamma_{2}}{a_{2}} \hat{E}_{2 p}>0$.

28) Die steigung der $\dot{\mathrm{p}}_{2}=0$-Kurve ist negativ: $\frac{\mathrm{dp}_{2}}{\mathrm{dS}_{2}}=\frac{\mathrm{u}_{\mathrm{S}} \mathrm{s}}{\zeta+\mathrm{x}_{2}}<0$.

29) Dies folgt aus der Eindeutigkeit der Lösung, d.h. die Trajektorien schneiden sich nicht. 


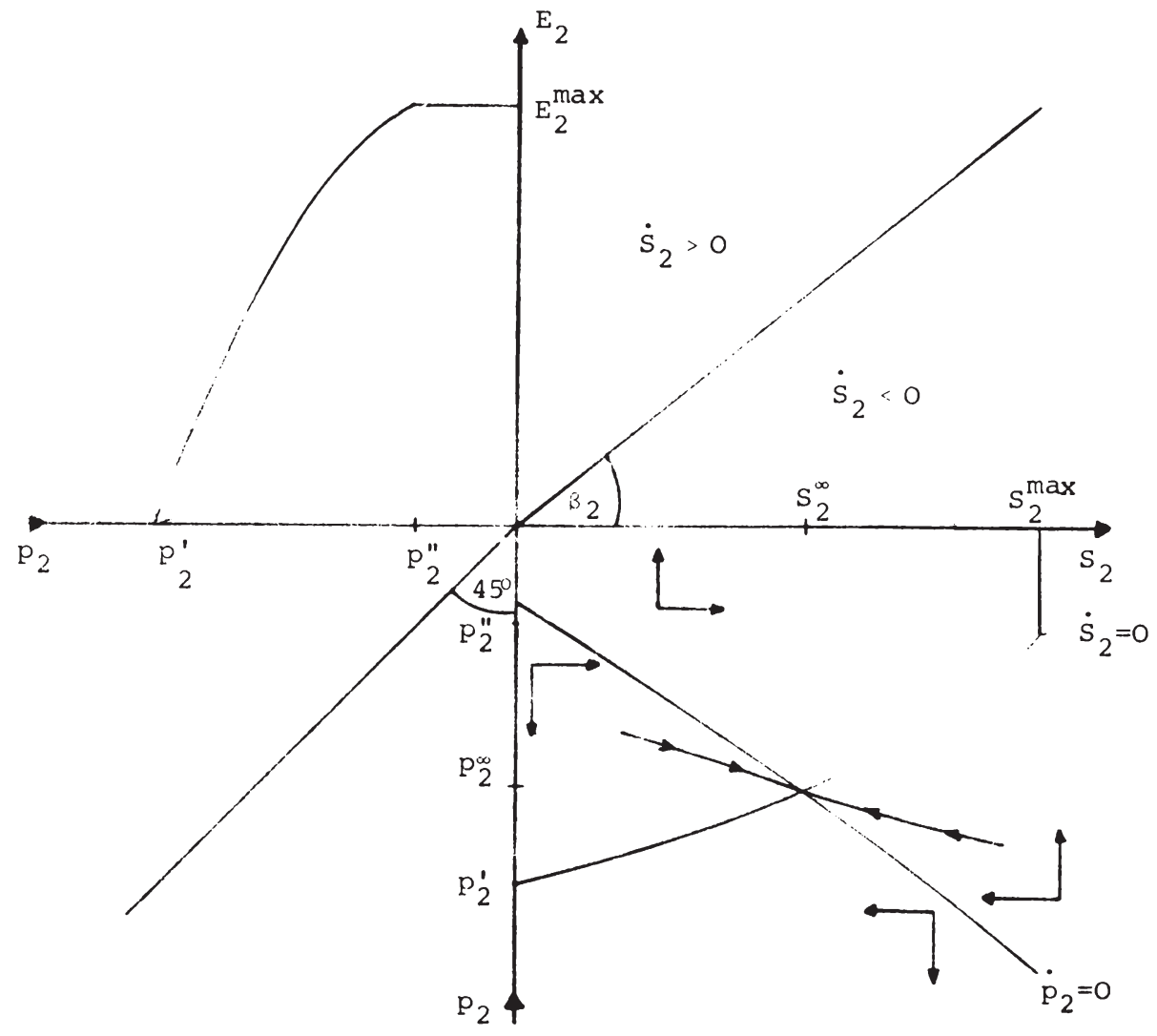

Schaubild 4: Die Bewegungseigenschaften des DGS für Region 2

(18) $\mathrm{p}_{1}=\frac{\mathrm{u}_{\mathrm{s}}^{1}}{\zeta+\alpha_{1}} 30$ )

Für die $\dot{S}_{1}=0$-Kurve kann kein bestimmter Verlauf mehr angegeben werden, da der stationäre ökologische zustand mit von den Emissionen der Region 2 abhängt. ${ }^{31)}$

30) $\mathrm{zu}$ den Eigenschaften vgl. Gleichung (17).

31) Vgl. Schaubild 3. 


$$
s_{1}=\frac{1}{\alpha 1}\left[\hat{E}_{1}\left(p_{1}\right)+\gamma_{2} \hat{E}_{2}\left(p_{2}\right)\right]
$$

In Schaubild 5 sind drei verschiedene Verläufe von $\dot{S}_{1}=0$ eingezeichnet, je nachdem wie groß $\mathrm{E}_{2}$ ist:

1. Fall: $E_{2}=0$

Für $\dot{\mathrm{s}}_{1}$ ergibt sich:

und für $\dot{S}_{1}=0$

$$
\dot{s}_{1}=\dot{E}_{1}\left(p_{1}\right)-\alpha_{1} s_{1}
$$

$$
S_{1}=\frac{1}{c_{1}} \hat{E}_{1}\left(p_{1}\right) \text { mit } \frac{d S_{1}}{d p_{1}}=\frac{1}{\alpha_{1}} \hat{E}_{1 p}>0
$$

d.h. der stationäre ökologische zustand ist nur von der verschmutzung und der Emission in Region 1 abhängig.

2. Fall: $E_{2}=E_{2}^{*} \in\left(O, E_{2}^{\max }\right)$ konstant Für $\dot{S}_{1}=0$ er:hält man

$$
\begin{aligned}
& S_{1}=\frac{1}{\alpha_{1}}\left[\hat{E}_{1}\left(p_{1}\right)+\gamma_{2} E_{2}^{*}\right] \\
& \text { mit } \frac{\partial S_{1}}{\partial p_{1}}=\frac{1}{\alpha_{1}} \hat{E}_{1 p}>0
\end{aligned}
$$

d.h. der stationäre ökologische zustand der Region ist abhängig von den Emissionen der Region 2. Da $\frac{\partial S_{1}}{\partial E_{2}^{*}}=\frac{r_{2}}{\alpha_{1}}>0$, ist $S_{1}$ umso größer, je größer $E_{2}^{*}$ ist.

3. Fall: $E_{2}:=E_{2}^{\max }$

Für $\dot{\mathrm{S}}_{1}=0 \mathrm{gilt}$ dann

$$
\begin{aligned}
& s_{1}=\frac{1}{\alpha_{1}}\left[\hat{E}_{1}\left(p_{1}\right)+\gamma_{2} E_{2}^{\max }\right] \\
& \text { mit } \frac{d i \frac{1}{d}}{p_{1}}=\frac{1}{\alpha_{1}} \hat{E}_{1 p} .
\end{aligned}
$$

Zusammenfassend läßt sich sagen, daß je größer $E_{2}$ ist, desto weiter nach rechts verschiebt sich die $\dot{s}_{1}=0$-Kurve. Diese Verschiebung erfolgt parallel, da im $\left(p_{1}, s_{1}\right)$-Diagramm die $\dot{S}_{1}=0$-Kurven jeweils die gleiche Steigung haben. Ist z.B. $E_{2}=E_{2}^{\max }, \mathrm{d} . \mathrm{h}$. in Region 2 wird die Steuer zu niedrig gesetzt bzw. es wird keine Umweltpolitik betrieben, dann muß in Region 1 eine stringente Umweltpolitik betrieben werden, um ins Optimum zu gelangen. Jedoch sind dann die Immissionen hoch, während der Güteroutput, aufgrund 
niedriger Emissionen, d.h. einer hohen Steuer, relativ klein ist. Ist dagegen $\mathrm{E}_{2}=0, \mathrm{~d} . \mathrm{h}$. in Region 2 wird eine sehr strenge Umweltpolitik betrieben, kann in Region 1 ein Wohlfahrtsoptimum verwirklicht werden, in dem die Immissionen relativ niedrig sind, während die Emissionen hoch sind und damit ein relativ großer Güteroutput erstellt werden kann.

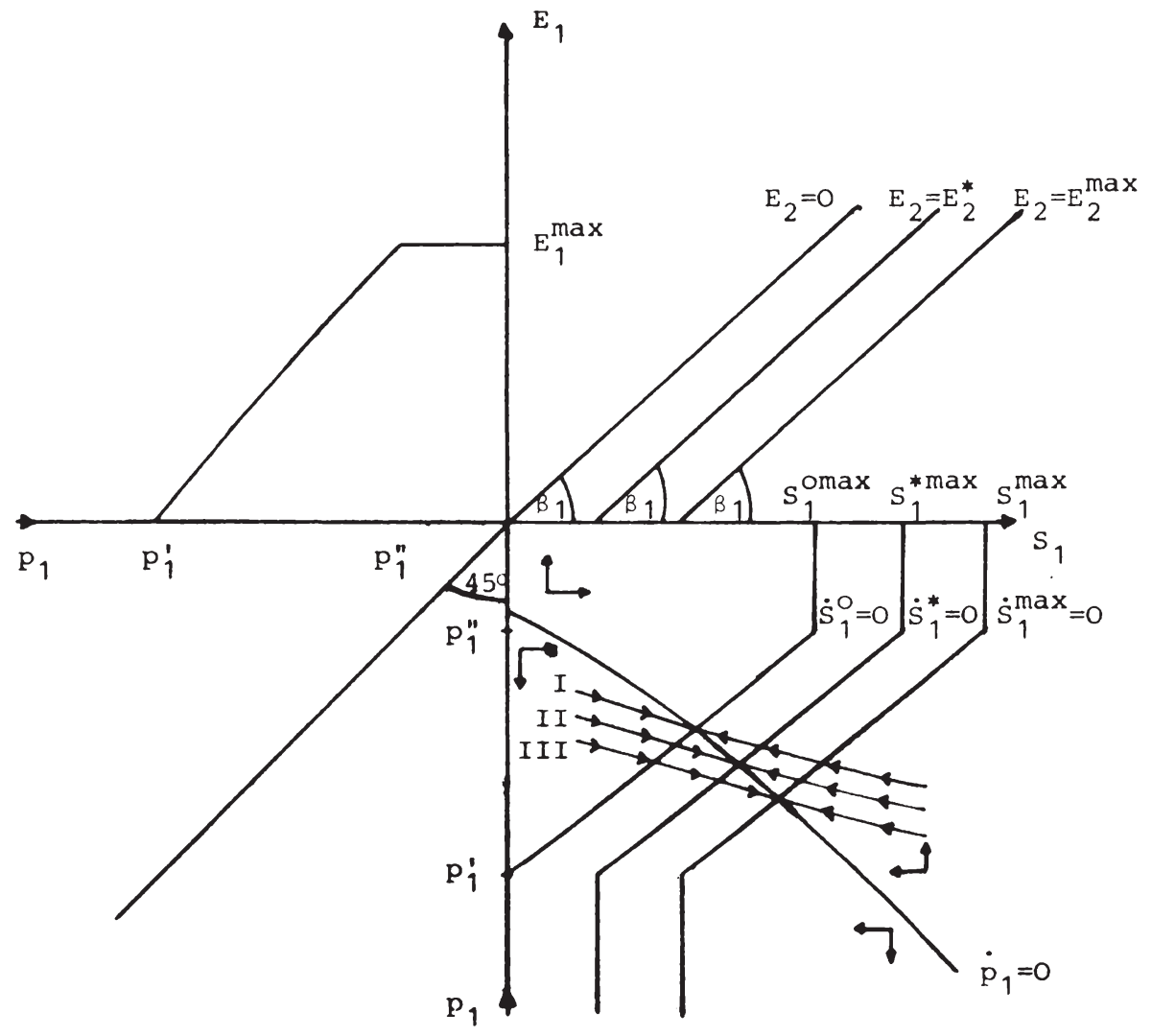

Schaubild 5: Phasendiagramm für Region 1 
Es ist ersichtlich, daß für $E_{1}=0$ die $\dot{S}_{1}=0-K u r v e$ nur dann die $\mathrm{p}_{1}$-Achse schneidet, wenn auch $\mathrm{E}_{2}=0$ ist. Ansonsten ist immer noch eine Verschmutzung der Region 1 vorhanden, wenn $E_{2}>0$ ist, auch wenn $\mathrm{E}_{1}=0$ ist, d.h. $\mathrm{p}_{1} \leq \mathrm{p}_{1}^{\prime}$ ist.

Der optimale Anfangswert des Immissionsschattenpreises $p_{1}$ variiert c.p. mit der Anfangsverschmutzung in Region 1 sowie der "importierten" Emissionen aus Region 2:

a) bezüglich der Anfangsverschmutzung muß der Schattenpreis ${ }^{32)}$ $p_{1}(0) c . p$. umso niedriger (höher) sein, je größer (kleiner) die Anfangsverschmutzung ist,

b) die Emissionen der Region 2 hängen vom Immissionsschattenpreis $p_{2}$ der Region $2 \mathrm{ab}$. Je höher $\mathrm{p}_{2}$ ist, $\mathrm{d} . \mathrm{h}$. je mehr in Region 2 emittiert wird, desto mehr Emissionen gelangen nach Region 1 , so daß $\mathrm{p}_{1}(\mathrm{O})$ niedrig gesetzt werden muß. Ist andererseits $\mathrm{p}_{2}$ niedrig, es wird in Region 2 also wenig emittiert, muß $p_{1}(0)$ hoch gesetzt werden. Dies bedeutet aber, daß die Umweltpolitik der Region 1 von der Umweltpolitik der Region 2 abhängig ist.

4.2.1 Die zeitliche Entwicklung der Emissionen in Region 2

Der Entwicklungspfad der Emissionen in Region 2 hängt davon ab, ob die Ausgangsverschmutzung $s_{2}^{\circ}$ größer, gleich oder kleiner als die stationäre Immission $\mathrm{S}_{2}^{\infty}$ ist:

Aus Gleichung (14) und den Überlegungen zu Gleichung (17) erhält man :

(20) $\dot{\mathrm{E}}_{2}=\hat{\mathrm{E}}_{2 \mathrm{p}} \dot{\mathrm{p}}_{2}: \begin{cases}>0 & \text { für } \mathrm{S}_{2}^{\circ}>\mathrm{S}_{2}^{\infty} \\ =0 & \text { für } \mathrm{S}_{2}=\mathrm{S}_{2}^{\infty} \\ <0 & \text { für } \mathrm{s}_{2}<\mathrm{S}_{2}^{\infty}\end{cases}$

32) $\mathrm{p}_{1}(t)<0 \forall t$. Vgl. zur Interpretation der Kovariablen Arrow $(1968,87-88)$. 
Zur Veranschaulichung dieser Differentialgleichung dient folgendes Schaubild:

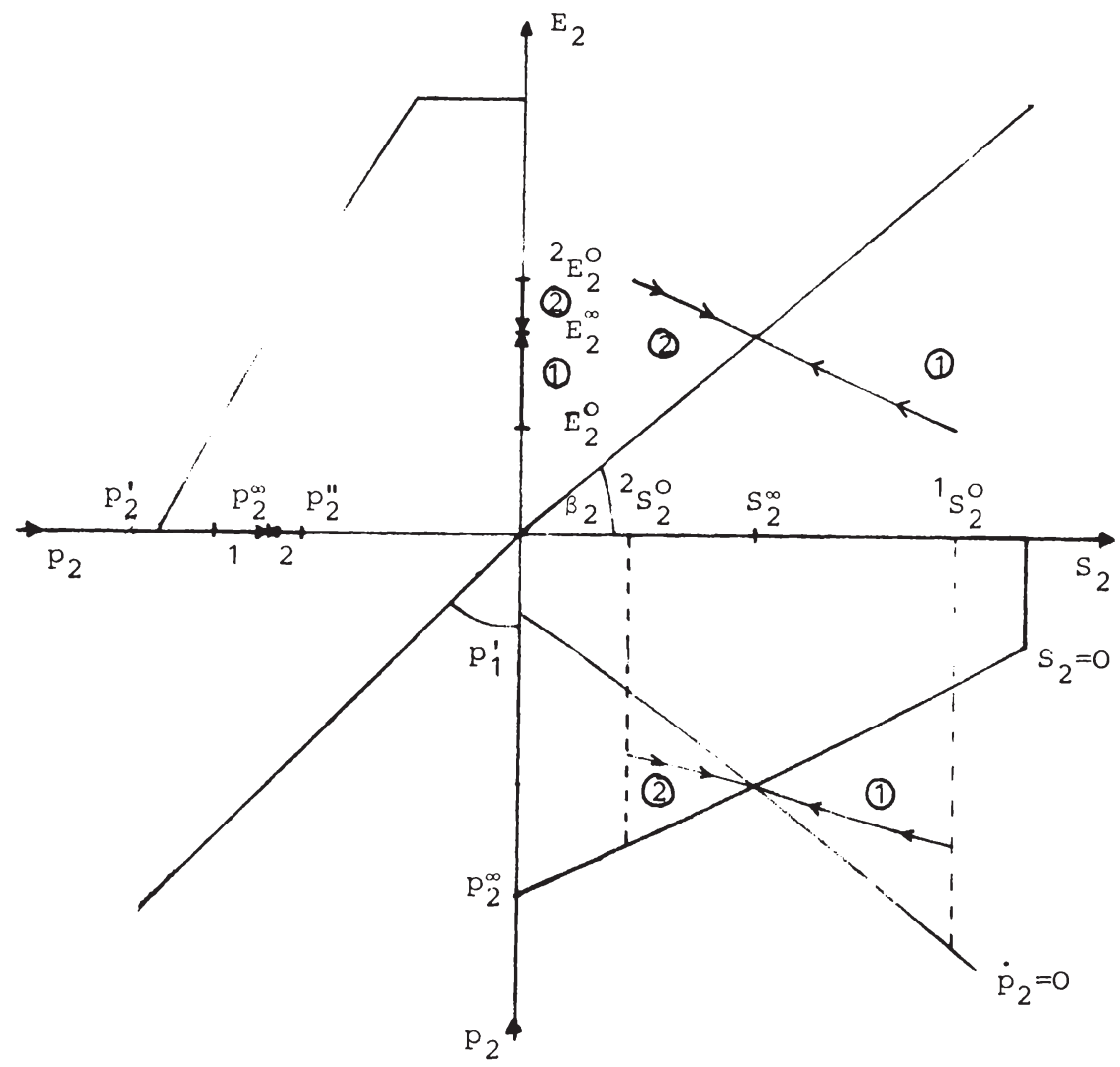

Schaubild 6: Bewegung der Emissionen in Region 2

Aus Schaubild 6 erkennt man, daß für $S_{2}={ }^{1} S_{2}^{0}>S_{2}^{\infty}$ der Immissionsschattenpreis $\mathrm{p}_{2}(0)$ so niedrig gesetzt wird, daß die Emissionen relativ niedrig sind $\left({ }^{1} E_{2}^{0}\right), d . h$. die Güterproduktion ist relativ klein. Durch diese niedrigen Emissionen verbessert sich im Zeitablauf die Umweltqualität, d.h. die Immission nimmt ab. Dadurch kann wieder mehr emittiert werden, d.h. die produktion in Region 2 wächst im Zeitablauf mit steigendem $p_{2}$. Dieser Proze $B$ kommt 
zum Stillstand, wenn das stationäre Gleichgewicht $\left(\mathrm{S}_{2}, \mathrm{p}_{2}\right)$ erreicht ist ( $v g l$. die pfeile 1 in Schaubild 6). Ist andererseits $s_{2}^{\circ}={ }^{2} S_{2}^{\circ}<S_{2}^{\infty}$ ergeben sich die analogen Anpassungen (vgl. die Pfeile 2 in Schaubild 6).

4.2.2 Die weitere zeitliche Entwicklung der Region 1

Aus den Gleichungen (17), (18) und (19) erhält man die Differentialgleichung

$$
\dot{S}_{1}^{*}=\frac{\gamma_{2}\left(\zeta+\alpha_{1}\right)}{\alpha_{1}\left(\zeta+\alpha_{1}\right)-u_{S S}^{T} \hat{E}_{1 p}} \dot{E}_{2} \quad \begin{cases}>0 & \text { für } s_{2}^{\circ}>s_{2}^{\infty 33)} \\ =0 & \text { für } s_{2}^{\circ}=s_{2}^{\infty} \\ <0 & \text { für } s_{2}^{\infty}<s_{2}^{\infty}\end{cases}
$$

und aus (18) und (21) erhält man

$$
\dot{p}_{1}^{*}=\frac{u_{s}^{1}}{\zeta+\alpha} \dot{s}_{1}^{*} \quad \begin{cases}<0 & \text { für } \left.s_{2}^{\circ}>s_{2}^{\infty} 34\right) \\ =0 & \text { für } s_{2}^{0}=S_{2}^{\infty} \\ >0 & \text { für } s_{2}<S_{2}^{\infty}\end{cases}
$$

wobei $\left(S_{1}^{*}, p_{1}^{*}\right)$ "Quasi-Steady-States" symbolisieren, d.h. "Steady States", die bei jeweils festen $E_{2}$ erreicht würden, wenn sich Region 1 auf den jeweiligen "Optimaltrajektorien" dorthin bewegt. Da sich jedoch $E_{2}$ ändern kann, stimmen diese Quasi-steady-states nur dann mit dem stationären Gleichgewicht $\left(S_{1}^{\infty}, \mathrm{p}_{1}^{\infty}\right)$ überein, wenn die Ausgangsverschmutzung in Region 2 mit der stationären Verschmutzung übereinstimmt $\left(s_{2}^{O}=s_{2}^{\infty}\right)$.

Geht man von $S_{2}^{\circ}>S_{2}^{\infty}$ im Zeitpunkt $O$ aus, ${ }^{35)}$ so ergibt sich aus (21), daß der Quasi-Steady-State-Wert der Immissionen in Region 1 im Zeitpunkt $O$ kleiner ist als der stady state Wert $s_{1}^{\infty}$, d.h. $S_{1}$ 33) $\mathrm{Da} \frac{\gamma_{2}\left(\zeta+\alpha_{1}\right)}{\alpha_{1}\left(\zeta+\alpha_{1}\right)-u_{S S}^{1} \hat{E}_{1 p}}>0$.

34) $\mathrm{Da} \frac{\mathrm{u}_{\mathrm{S}}^{1}}{\zeta+\alpha_{1}}<0$.

35) Davon soll bei der weiteren Diskussion immer ausgegangen werden. Für $S_{2}^{0}=S_{2}^{\infty}$ ergibt sich keine Verschiebung von $\left(p_{1}^{\infty}, S_{1}^{\infty}\right)$. Für $S_{2}<S_{2}^{\infty}$ ist die Diskussion analog. 
steigt im Zeitablauf solange, bis $\dot{\mathrm{E}}_{2}=0$ ist. Aus (22) erhält man die zeitliche Entwicklung des Quasi-Steady-State-Wertes des Immissionsschattenpreises $p_{1}$. Dieser sinkt im Zeitablauf solange, bis $S_{2}^{\infty}$ erreicht ist, d.h. Region 2 sich im Steady state befindet. Dieser zeitliche Ablauf wird in Schaubild 7 dargestellt; wobei die Pfeile die Bewegungsrichtung angeben.

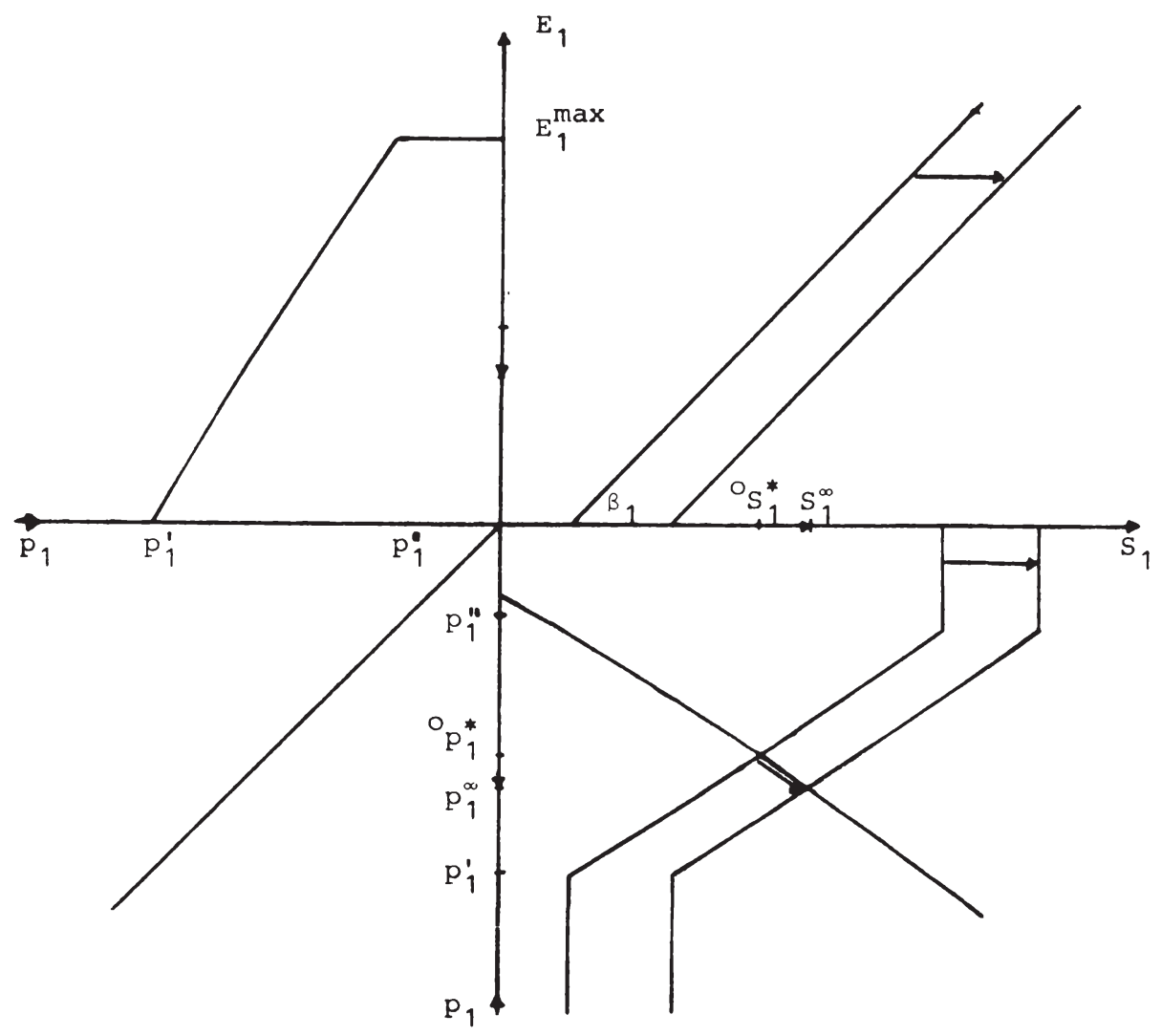

Schaubild 7: Die zeitliche Entwicklung des Quasi-Steady-State-Wertes 
Je mehr $E_{2}$ aliso steigt, desto weiter verschiebt sich die $\dot{S}_{1}=0-$ Kurve parallel nach außen, d.h. auch ihr Schnittpunkt mit der $\dot{\mathrm{p}}_{1}=$-Kurve verschiebt sich nach rechts unten, so daß die Optimaltrajektorien sich ebenfalls nach unten verschieben. Da die Regionalbehörde der Region 1 die importierten Emissionen aus Region 2 jweils als gegeben annehmen, past sich der Immissionsschattenpreis der Region 1 durch den Ubergang von der Ausgangstrajektorie $z u$ einer $z u$ dem neuen Quasi-Steady-State führenden Trajektorie unmittelbar an. Im zeitkontinuierlichen Fall gibt es unendlich viele solcher Anpassungen (solange bis $\dot{E}_{2}=0$ ist), so daß man zu einer "neuen" Trajektorie kommt, die im folgenden Reaktionstrajektorie genannt wird.

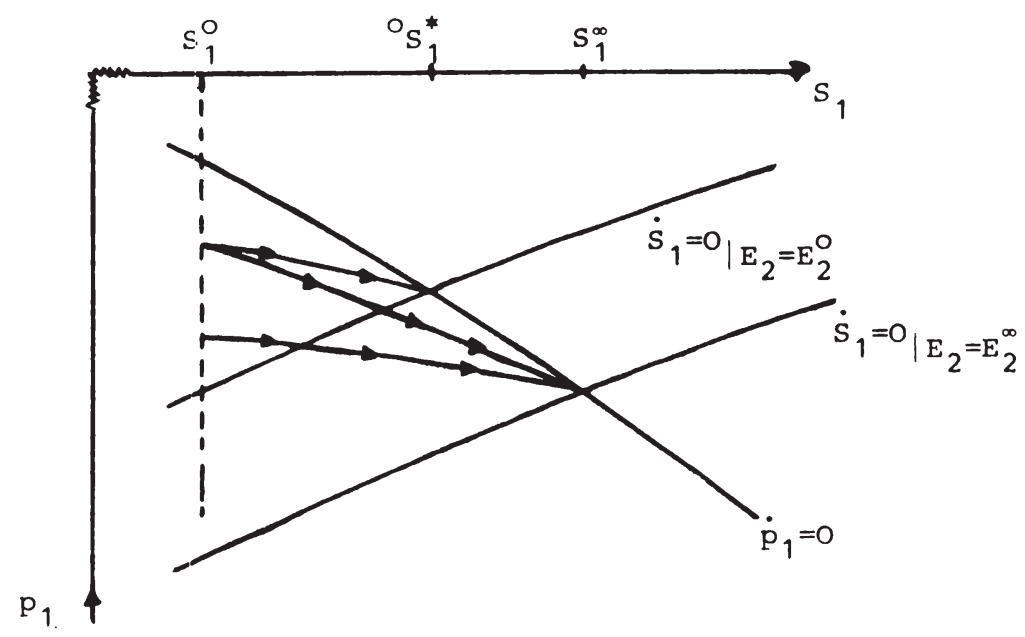

Schaubild 8: Die Reaktionstrajektorie

In Schaubild 8 ist solch eine Reaktionstrajektorie dargestellt unter den Annahmen:

(i) $s_{2}^{\circ}>S_{2}^{\infty}$, d.h. $\dot{E}_{2}>0$ und damit ${ }^{\circ} s_{1}^{*}<s_{1}^{\infty} \cdot{ }^{36)}$

36) Diese Annahme soll auch weiterhin gelten. 
(ii) $\mathrm{S}_{1}^{\circ}<{ }^{\circ} \mathrm{S}_{1}^{*}$, d.h. die Ausgangsverschmutzung in Region 1 ist kleiner als der stationäre Wert, der über die Optimaltrajektorie erreicht würde, wenn $\dot{\mathrm{E}}_{2}=0$ wäre.

In diesem 1. Fall müssen die Emissionen in Region 1 mehr abnehmen, um zu $S_{1}^{\infty} z u$ gelangen, als um zu ${ }^{\circ} S_{1}^{*} z u$ gelangen. Dies bedeutet, da im Optimum Vollbeschäftigung herrscht, daß der Güteroutput stärker zurückgehen muß. Gleichzeitig steigen jedoch die Immissionen, so daß, durch die hohe Umweltverschmutzung in Region 2, Region 1 Wohlfahrtsverluste erleidet.

2. Fall: $\mathrm{S}_{1}^{\circ}={ }^{\circ} \mathrm{S}_{1}^{*}$

d.h. Region 1 befindet sich in der Ausgangslage in einem QuasiSteady-state. Da $E_{2}$ kontinuierlich zunimmt, entwickelt sich Region 1 entlang der $\dot{p}_{1}=0$-Kurve in Richtung zum steady state $\left(s_{1}^{\infty}, p_{1}^{\infty}\right)$.

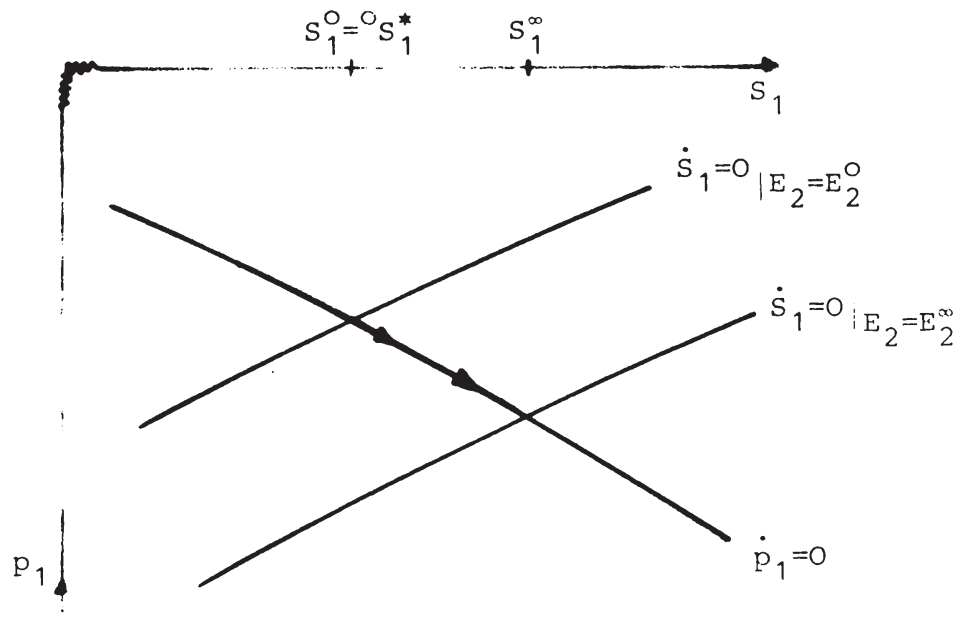

Schaubild 9: Reaktionstrajektorie für $S_{1}^{\circ}={ }^{\circ} S_{1}^{*}$

Region 1 befindet sich also immer in einem Quasi-Gleichgewicht, während die Immissionen zunehmen und die Emissionen, und damit 
auch die Güterproduktion, abnehmen. Region 1 erleidet dadurch Wohlfahrtsver:uste, während in Region 2 Wohlfahrtsgewinne zu verzeichnen sind (die Güterproduktion und die Umweltqualität erhöht sich).

3. Fall: $\mathrm{S}_{1}^{\mathrm{O}}>{ }^{\mathrm{O}} \mathrm{S}_{1}^{*}$

d.h. die Immissionen in der Ausgangslage sind höher als die Immissionen, die erreicht würden, wenn $\dot{\mathrm{E}}_{2}=0$ wäre. Da aber $\dot{\mathrm{E}}_{2}>\mathrm{O}$ ist und damit ${ }^{O} \mathrm{~S}_{1}^{*}<\mathrm{S}_{1}^{\infty}$ kommt es auf die Höhe von $S_{1}^{\circ}$ im Vergleich zur Höhe von $\mathrm{S}_{1}^{\infty}$ an, welchen Verlauf die Reaktionstrajektorie hat.

Ist $S_{1}^{\circ}=S_{1}^{\infty}$, dann werden die Immissionen in Region 1 zuerst abnehmen, und zwar solange, bis $s_{1}=\widetilde{S}_{1}^{*}$ ist, d.h. Region 1 sich in einem Quasi-Steady-State befindet. Solch ein $\left(\tilde{\mathrm{S}}_{1}^{*}, \tilde{\mathrm{p}}_{1}^{*}\right)$ wird erreicht, da sich die Emissionen in Region 2 kontinuierlich erhöhen und damit in der Entwicklung der Immissionen der Region 1 im Zeitablauf kein "Sprung" vorhanden sein kann. Ist $\left(\tilde{S}_{1}^{*}, \tilde{p}_{1}^{*}\right)$ erreicht, befinden wir uns wieder im 2. Fall: Die Immissionen erhöhen sich bei sinkenden Emissionen, d.h. sinkender Güterproduktion wieder auf den gleichen Wert wie in der Ausgangslage. Durch den "Import" der Emissionen aus Region 2 erleidet Region 1 also Wohlfahrtsverluste dadurch, daß sich trotz Umweltpolitik die Immissionen gegenüber der Ausgangslage nicht ändern, aber die Giuterproduktion als Folge der erhobenen steuer zurückgeht.

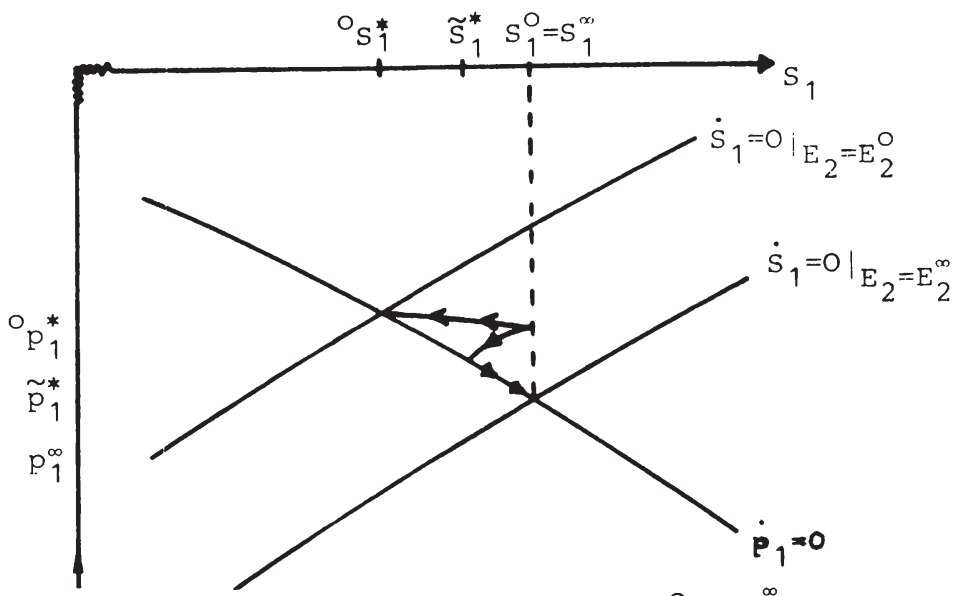

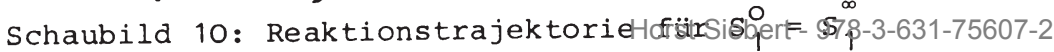


Ist ${ }^{O} S_{1}^{*}<S_{1}^{O}<S_{1}^{\infty}$, ist die Entwicklung analog $z u$ der im letzten Abschnitt $\left(S_{1}^{\circ}=S_{1}^{\infty}\right)$. Allerdings ist, da $S_{1}^{0}<s_{1}^{\infty}$, die Immission im Steady State größer als in der Ausgangslage. Also auch in diesem Fall muß Region 1 Wohlfahrtsverluste hinnehmen.

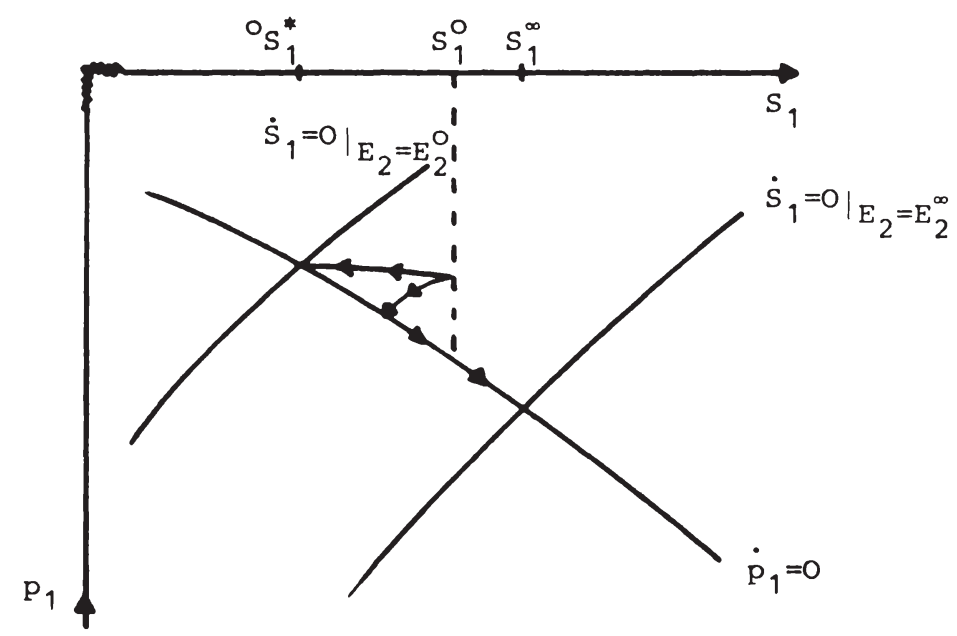

Schaubild 11: Reaktionstrajektorie für ${ }^{\circ} S_{1}^{*}<s_{1}^{0}<s_{1}^{\infty}$

Ist $S_{1}^{0}>S_{1}^{\infty}$, kann keine eindeutige Aussage über den Verlauf der Reaktionstrajektorie gemacht werden. Je größer $s_{1}^{0}$ ist, desto mehr ist zu erwarten, daß die Reaktionstrajektorie c.p. "direkt" zum steady-state-Wert geht und umgekehrt, je näher $s_{1}^{\circ}$ bei $s_{1}^{\infty}$ liegt, desto eher ist c.p. zu erwarten, daß die Reaktionstrajektorie einen ähnlichen Verlauf nimmt, wie für $s_{1}^{0}=s_{1}^{\infty}$. Für $S_{1}^{O}>S_{1}^{\infty}$ macht Region 1 also gegenüber der Ausgangssituation $\left(s_{1}^{0}, p_{1}(0)\right)$ Wohlfahrtsgewinne, da sowoh1 die Immission sinkt als auch die Güterproduktion steigt. Jedoch werden diese Wohlfahrtsgewinne durch die steigenden Emissionen aus Region 2 abgeschwächt. (Vgl. $s_{1}^{*}$ und $\left.s_{1}^{\infty}\right)$. 


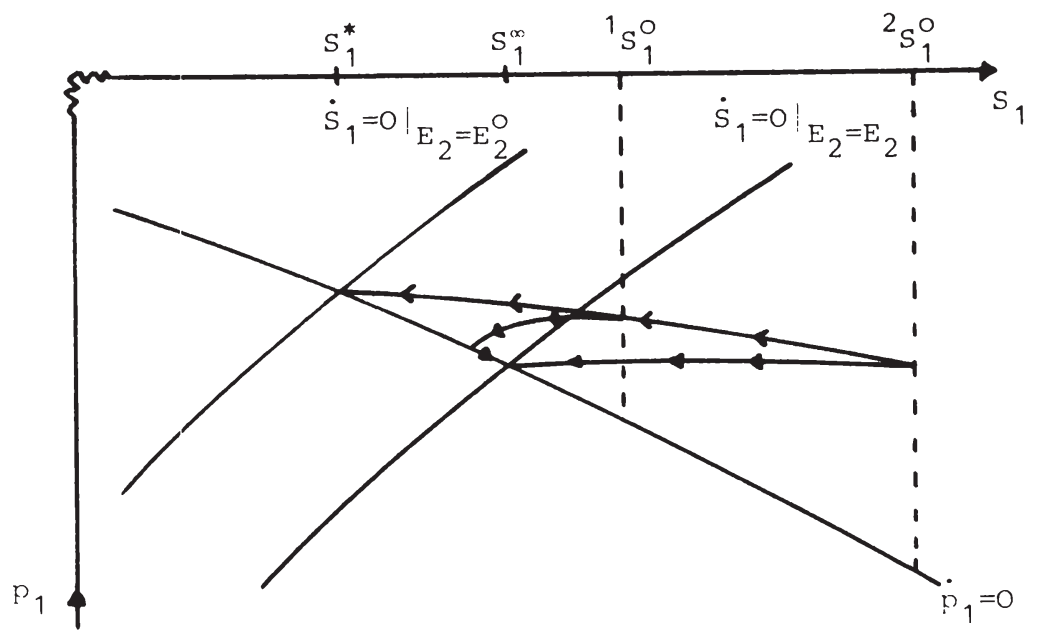

Schaubild 12: Reaktionstrajektorien für $S_{1}^{\circ}>S_{1}^{\infty}$

\section{Gegenseitige Verschmutzung}

Gehen wir jetzt von dem Fall aus, daß sowohl Region 1 als auch Region 2 einen Teil ihrer Emissionen an die jeweils andere Region abgibt. ${ }^{37)}$ Es wird mit Hilfe der Theorie der open-loop Differentialspiele gezeigt, daß ein Steady-State exisitert, wenn sich die Regionalbehörden wie Cournot-oligopolisten (StackelbergNachfolger) verhalten, ${ }^{38)} \mathrm{d} . \mathrm{h}$. die "importierten" Emissionen werden jeweils als gegeben betrachtet.

Die optimierungsprobleme beider Regionalbehörden lauten dann folgendermaßen $(i, j=1,2 ; i \neq j)$

$\operatorname{Max} w^{i}=u^{i}\left(x_{i}, s_{i}\right) e^{-\zeta t} d t$

u.d.B. (1), (2), (3), (4)

$x_{i}, R_{i}, E_{i} S_{i} \geqq 0 \quad \forall t \in[0, \infty)$

$S_{i}(O)=S_{i}^{O}$ gegeben

$\zeta$ gegeben und positiv

37) Vgl. Schaubild 1

38) Zum Modellansatz vgl. Bernstein (1977). Van Zele (1978) untersucht diese Fragestellung in statischem Kontext. 


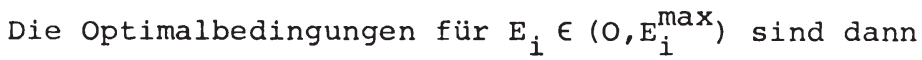

$$
\begin{aligned}
& p_{i}\left(1-\gamma_{i}\right)+u_{x}^{i} f_{E}^{i}=0 \\
& \dot{p}_{i}=\left(\zeta+\alpha_{i}\right) p_{i}-u_{S}^{i} \\
& \dot{s}_{i}=\left(1-\gamma_{i}\right) E_{i}+\gamma_{j} E_{j}-\alpha_{i} s_{i}
\end{aligned}
$$

In Analogie zu Gleichung (13) bzw. (14) können folgende Funktionen abgeleitet werden:

$$
E_{i}=\hat{E}_{i}\left(p_{i}\right):\left\{\begin{array}{c}
E_{i}=0 \quad \forall p_{i} \leqq p_{i}^{\prime}, p_{i}^{\prime} \equiv-\left.\frac{u_{x}^{i} f_{E}^{i}}{1-\gamma_{i}}\right|_{E_{i}=0} \\
E_{i}>0, \hat{E}_{i p}>0 \underset{i}{\forall p_{i}} \epsilon\left(p_{i}^{\prime}, p^{\prime}{ }_{i}\right) \\
p_{i} \equiv-\frac{u_{x}^{i} f_{E}^{i}}{1-\gamma} \mid E_{i}=E_{i}^{\max } \\
E_{i}=E_{i}^{\max } \forall p_{i} \geqq p_{i}^{\prime \prime}
\end{array}\right.
$$

Im folgenden wollen wir davon ausgehen, daß Randlösungen ausgeschlossen sind, d.h. es soll gelten $p_{i} \in\left(p_{i}^{\prime}, p_{i}^{\prime \prime}\right)$. Da $\hat{E}_{i}$ in diesem Bereich streng monoton wachsend ist, existiert die Umkehrfunktion:

$$
p_{i}=\hat{E}_{i}^{-1}\left(E_{i}\right)
$$

Im Steady state $\dot{p}_{i}=0, \dot{s}_{i}=0$ gilt

$$
\begin{aligned}
& \left(\zeta+\alpha_{i}\right) p_{i}-u_{S}^{i}=0 \\
& \left(1-\gamma_{i}\right) E_{i}-\alpha_{i} S_{i}=-\gamma_{j} E_{j}
\end{aligned}
$$

Daraus können Reaktionsfunktionen $\varepsilon_{i}, \sigma_{i}$ abgeleitet werden mit $E_{i}=\varepsilon_{i}\left(E_{j}\right)$ und $S_{i}=\sigma_{i}\left(E_{j}\right)$ :

Einsetzen von (26) und totale Differentation nach $E_{j}$ ergibt

$$
\left(\begin{array}{ccc}
\left(\zeta+\alpha_{i}\right) \hat{E}_{i E}^{-1} & -u_{S S}^{i} \\
\left(1-\gamma_{i}\right) & -\alpha_{i}
\end{array}\right)\left(\begin{array}{l}
\frac{d E_{i}}{d E_{j}} \\
\frac{d S_{i}}{d E_{j}}
\end{array}\right)=\left(\begin{array}{l}
0 \\
-\gamma_{j}
\end{array}\right)
$$

Die Steigungen sind dann nach der Cramér'schen Regel 
(30)

$$
\frac{d E_{i}}{d E_{j}}=\varepsilon_{i E}=\frac{-\gamma_{j} u_{S S}^{i}}{\left(1-\gamma_{i}\right) u_{S S}^{i}-\alpha_{i}\left(\zeta+\alpha_{i}\right) \hat{E}_{i E}^{-1}}>0
$$

und

$$
\frac{d S_{i}}{d E_{j}}=\sigma_{i E}=\frac{-\gamma_{j}\left(\zeta+\alpha_{i}\right) \hat{E}_{i E}^{-1}}{\left(1-\gamma_{i}\right) u_{S S}^{i}-\alpha_{i}\left(\zeta+\alpha_{i}\right) \hat{E}_{i E}^{-1}}>0
$$

Da sich die Emissionen in beiden Regionen kontinuierlich ändern, ergibt sich ein Gesamt-Steady-State dann, wenn sowohl $E_{i}$ als auch $E_{j}$ stationär sind. Ein solches Steady-State kann folgendermaßen definiert werden:

$$
\varepsilon_{i}\left(E_{j}\right)=\varepsilon_{j}^{-1}\left(E_{j}\right)
$$

D.h. im Schnittpunkt der Emissions-Reaktionsfunktionen der Region $i$ und der Region $j$ befindet sich die ökonomie in einem Gleichgewicht. Um die Existenz eines solchen Gleichgewichts zu zeigen, muß man den Verlauf der Funktionen $\varepsilon_{i}$ und $\varepsilon_{j}$ kennen. Wir untersuchen die Funktion $\varepsilon_{i}$, für $\varepsilon_{j}$ gilt die analoge Argumentation. Die Steigung $\varepsilon_{i E}$ ist nach (30) kleiner als Null. Weiter kann gezeigt werden, daß

$$
\varepsilon_{i E}>-M_{i}=-\left(\gamma_{i}+\gamma_{j}\right)>-1
$$

ist, da laut voraussetzung $\gamma_{i}+\gamma_{j}<1$ ist. ${ }^{39)}$ Ist $E_{j}=0$, dann gibt $E_{i}=\varepsilon_{i}(0)$ den Steady-State-Wert der Emissionen von Region $i$ an, der erreicht würde, wenn keine importierten Emissionen $\mathrm{zu}$ berücksichtigen wären. Da $E_{i} \in\left(O, E_{i}^{\max }\right)$ gilt,

$$
\text { 39) } \begin{aligned}
& \varepsilon_{i E}=\frac{-\gamma_{j} u_{S S}^{i}}{\left(1-\gamma_{i}\right) u_{S S}^{i}-\alpha_{i}\left(\zeta+\alpha_{i}\right) \hat{E}_{i E}^{-1}} \stackrel{!}{j}-\left(\gamma_{i}+\gamma_{j}\right) \\
& \Leftrightarrow \gamma_{i}+\gamma_{j}-1<-\frac{\left(\gamma_{i}+\gamma_{j}\right) \hat{E}_{i E}^{-1} \alpha_{i}\left(\zeta+\alpha_{i}\right)}{\gamma_{i} u_{S S}^{i} \hat{E}_{i E}^{-1} \alpha_{i}\left(\zeta+\alpha_{i}\right)} \\
& \Leftrightarrow \gamma_{i}+\gamma_{j}-1<0<-\frac{\left(\gamma_{i}+\gamma_{j} \hat{E}_{i} u_{S S}^{i}\right.}{\gamma_{i}}
\end{aligned}
$$


folgt,

$$
O<\varepsilon_{i}(O)<E_{i}^{\max }
$$

Ist $E_{j}=E_{j}^{\max }$, so gilt wegen Bedingung

$\alpha_{i} S_{i \mid E_{i}}=0>\gamma_{j} E_{j}^{\max }$, daß

$$
\varepsilon_{i}\left(E_{j}^{\max }\right)>0
$$

Aus (30), (34) und (35) erhält man dann

$$
E_{i}^{\max }>\varepsilon_{i}(O)>\varepsilon_{i}\left(E_{j}\right) \mid E_{j} \in\left(O, E_{j}^{\max }\right)^{>\varepsilon_{i}}\left(E_{j}^{\max }\right)>0
$$

Jetzt kann folgendes Theorem, dessen Beweis im Anhang erfolgt, formuliert werden:

Theorem 1: Es existiert genau ein Gesamt-Steady-State.

Bis jetzt wurde gezeigt, daß ein Gesamtgleichgewicht existiert. Jetzt wollen wir den Anpassungspfad betrachten, wenn eine Region in der Ausgangslage einen hohen Immissionsbestand hat, während in der anderen Region relativ niedrige Immissionen sind. D.h. wir betrachten den Fall $S_{i}^{O}\left\langle S_{i}^{*}\right.$ und $S_{j}^{0}>S_{j}^{*}$. Da im Anfangszeitpunkt in Region $i$ die Immissionen kleiner als wohlfahrtsoptimal sind, wird von der Regionalregierung eine niedrige steuer gesetzt, so daß die Emissionen in $i$ relativ groß sind. Das bedeutet aber für Region $j$, daß ihr Ausgangsquasi -steady-State $S_{j}^{*}$ größer ist als $S_{j}^{\infty}$. Da annahmegemäß $S_{j}^{0}>S_{j}^{*}$, folgt, daß eine hohe steuer gesetzt wird, was wiederum bedeutet, daß $E_{j}^{O}$ niedrig ist. In Region $i$ gilt dann $S_{i}^{*}<S_{i}^{\infty}$. In Region $i(j)$ sinken (steigen) die Emissionen aufgrund der niedrigen (hohen) Steuer. Da dies simultan geschieht und die importierten Emissionen jeweils als gegeben angenommen werden, verschieben sich die Quasi-steady-States gemä $B$ folgender Funktionen: 
Die Ableitung des Reaktionsfunktionsgleichgewichts kann folgendermaßen graphisch erfolgen:
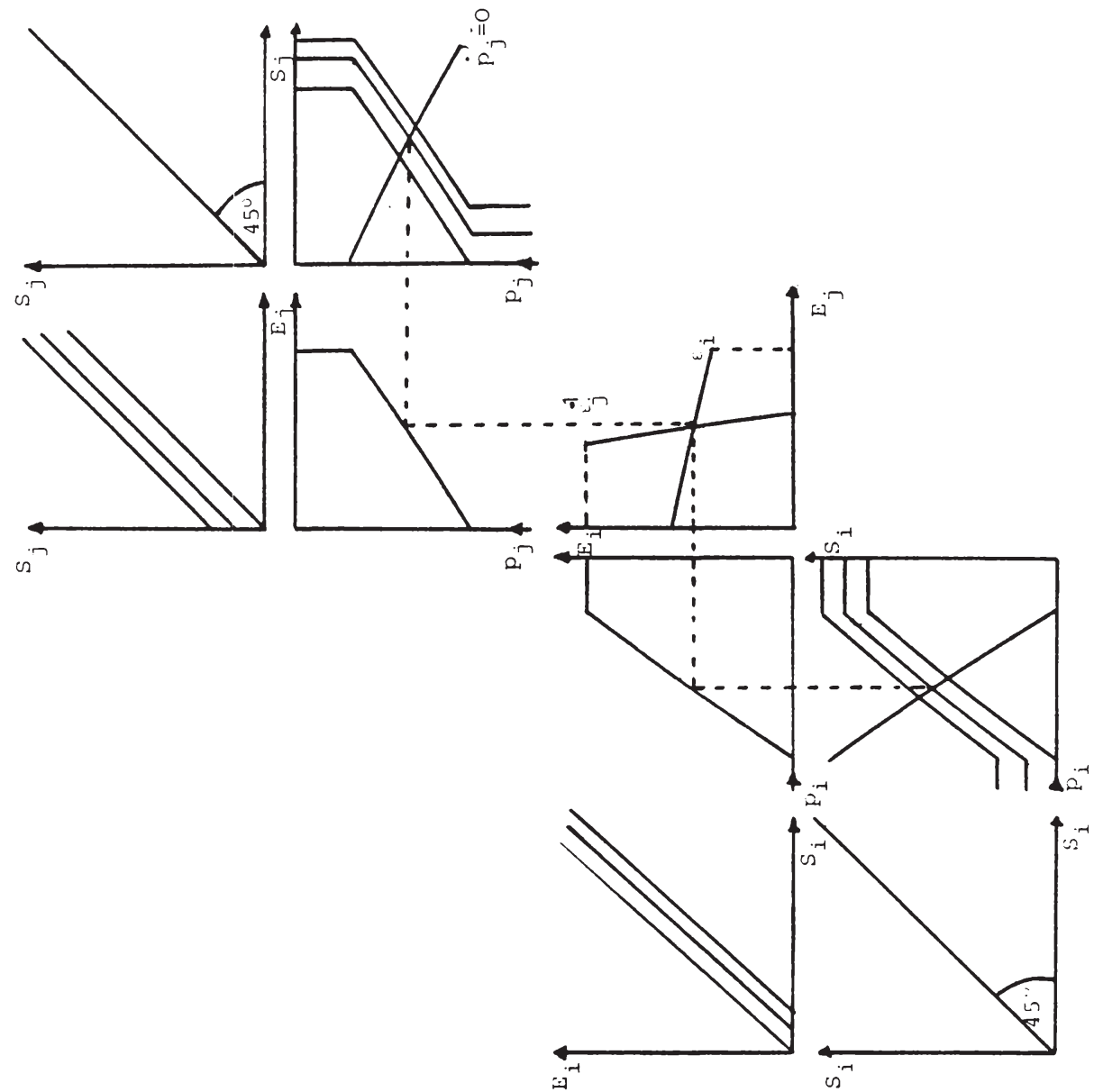

Schaubild 13: Die graphische Ableitung der Raktionsfunktionen $\varepsilon_{i}$ und $\varepsilon_{j}$. 


$$
\begin{aligned}
& \dot{S}_{k}^{*}=\frac{\gamma_{1}\left(\zeta+\alpha_{k}\right)}{\alpha_{k}\left(\zeta+\alpha_{k}\right)-\left(1-\gamma_{k}\right) u_{S S}^{k} \hat{E}_{k p}} \dot{E}_{1} \\
& \dot{p}_{k}^{*}=\frac{\gamma_{1} u_{S S}^{k}}{\alpha_{k}\left(\zeta+\alpha_{k}\right)-\left(1-\gamma_{k}\right) u_{S S}^{k} \hat{E}_{k p}} \hat{E}_{1}
\end{aligned}
$$

Das bedeutet aber, daß $S_{i}^{*}\left(S_{j}^{*}\right)$ größer (kleiner) wird, wenn die Emissionen in $j(i)$ steigen (fallen), während $p_{i}^{*}\left(p_{j}^{*}\right)$ sinkt (steigt). Damit verschieben sich auchdie Optimaltrajektorien, so daß man im vorliegenden zeitkontinuierlichen Fall zu Reaktionstrajektorien kommt, die gegen das Gesamt-Steady-State streben.

Aus Schaubild 14 ist ersichtlich, daß diejenige Region Wohlfahrtsverluste erleidet, die im Anfangszeitpunkt einen niedrigen Immissionsbestand hat, während die andere Wohlfahrtsgewinne zu verzeichnen hat. Durch die importierten Emissionen steigt in Region $i$ die Immission über das Quasi-Steady-state ${ }^{{ }_{S}}{ }_{i}^{*}$ hinaus, während die Emissionen stärker abnehmen und damit die produktion des Gutes $x_{i}$. Andererseits sinkt in Region $j$ die Umweltverschmutzung stärker, während die Produktion an Konsumgütern steigt. Ist in beiden Regionen der Immissionsbestand niedrig oder ist er in beiden hoch, kann ein solcher Anpassungsprozeß nicht gezeigt werden, da dann die Emissionen steigen, konstant bleiben oder fallen können und damit die Bewegung der Quasi-Steady-States im Zeitablauf unklar bleibt. 

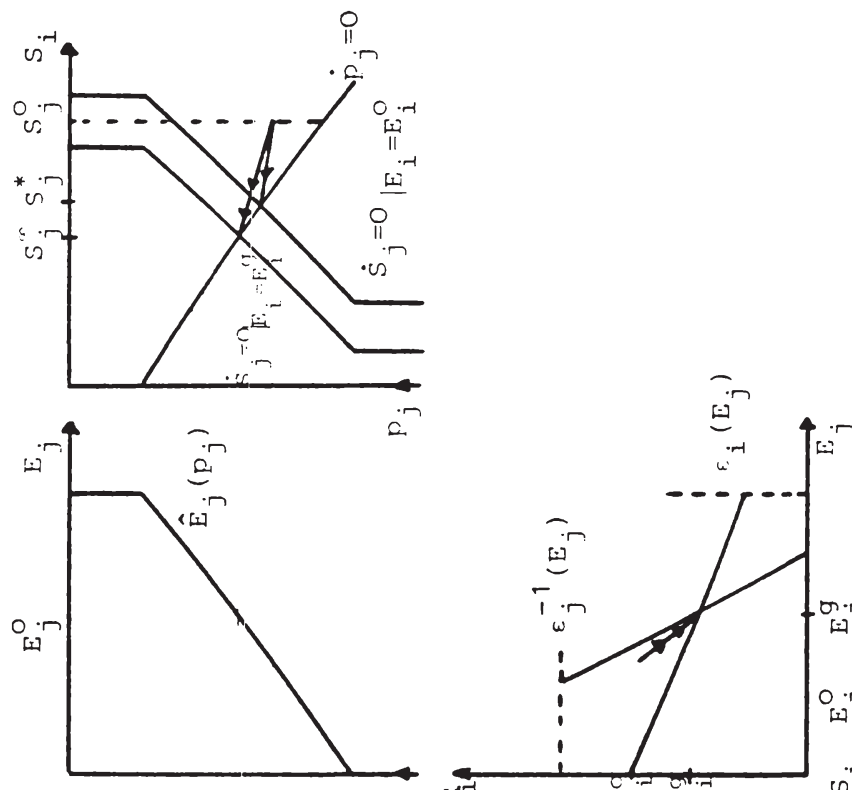

a.

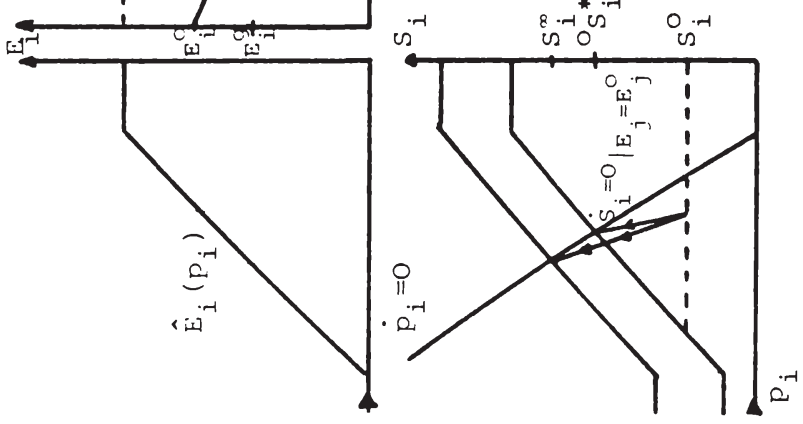

c.-1.

Schaubild 14: Anpassungspfade bei gegenseitiger Verschmutzung 
Anhang: Beweis von Theorem 1

Sei $A_{1}=\left\{E_{1} \mid E_{1} \in\left[O, E_{1}^{\max }\right]\right\} \quad 1=i, j$

Dann gilt

$$
\varepsilon_{i}: A_{i} \rightarrow A_{j} \text { bzw. } \varepsilon_{j}: A_{j} \rightarrow A_{i}
$$

Weiterhin sei $\varepsilon\left(E_{i}, E_{j}\right)=\left(\varepsilon_{i}\left(E_{j}\right), \varepsilon_{j}\left(E_{i}\right)\right)$ und $A_{i} \times A_{j} \equiv A$.

Für E gilt dann

$$
\varepsilon: A \rightarrow A
$$

A ist eine abgeschlossene Teilmenge des $R^{2}$ und damit ein vollständiger metrischer Raum.

Wir zeigen jetzt, $\mathrm{da} \beta \varepsilon: A \rightarrow A$ eine Kontraktion ist: Folgende Bedingungen müssen erfüllt sein:

$\left\|\varepsilon\left(E_{i}^{1}, E_{j}^{1}\right)-E\left(E_{i}^{2}, E_{j}^{2}\right)\right\| \leqq M\left\|\left(E_{i}^{1}, E_{j}^{1}\right)-\left(E_{i}^{2}, E_{j}^{2}\right)\right\| !$ wobei $R \ni M=\gamma_{i}+\gamma_{j},\left(E_{i}^{k}, E_{j}^{k}\right)^{i} A, k=1,2$ und $\|(x, y)\|_{\infty}=\max \{|x|,|y|\}$

Wegen des Mittelwertsatzes der Differentialrechnung gilt [Vgl.

Dieudonné $(1969,160)]$.

$$
\begin{aligned}
=\max \{ & \left.\frac{-\gamma_{j} u_{S S}^{i}}{\left(1-\gamma_{i}\right) u_{S S}^{i}-\alpha_{i}\left(\zeta+\alpha_{i}\right) \hat{E}_{i E}^{-1}}, \frac{-\gamma_{i} u_{S S}^{j}}{\left(1-\gamma_{j}\right) u_{S S}^{j}-\alpha_{j}\left(\zeta+\alpha_{j}\right) \hat{E}_{j E}^{-1}}\right\} \|\left(E_{i}^{1}, E_{j}^{1}\right)- \\
& \left.-\left(E_{i}^{2}, E_{j}^{2}\right)\left\|<\left(\gamma_{i}+\gamma_{j}\right)\right\|\left(E_{i}^{1}, E_{j}^{1}\right)-E_{i}^{2}, E_{j}^{2}\right) \|
\end{aligned}
$$

Daraus folgt, $\mathrm{da} \beta \varepsilon: A \rightarrow A$ eine Kontraktion ist. Dann gilt nach dem Kontraktionssatz [Vgl. Wloka (1971, 30)], daß $\varepsilon$ genau einen Fixpunkt besitzt.

Jetzt ist noch zu zeigen, daß dieser Fixpunkt nicht am Rand liegt, d.h. er liegt im Inneren von A. Dies folgt direkt aus Bedingung (36).

Q.E.D. 
Literaturverzeichnis:

D'Arge, R.C. and K.C. Kogiku (1973), Economic Growth and the Environment, Review of Economic Studies, 61-77.

Arrow, K.J. (1968), Applications of Control Theory to Economic Growth, in: G.B. Dantzig, A.F. Veinott Jr., Mathematics of the Decision Sciences, pt. 2, Providence, 85-119.

Bernstein, J.E. (1977), A Noncooperative Model of Public Investment and International Externalities, International Economic Review 18, 393-406.

Bonus, M. (1972), Über Schattenpreise von Umweltressourcen, Jahrbuch für Sozialwissenschaft 23, 342-354.

Brookshire, D.S. (1978), A Macroeconomic Analysis of Regional Environmental Modeling and Planing, Journal of Environmental Economics and Management 5, 268-282.

Comolly, P.M. (1975), Dynamic General-Equilibrium Models in Environmental Economics, Ph.D. dissertation, Purdue University, West Lafayette, Ind..

Converse, A.O. (1974), Environmental Controls and Economic Growth, Journal of Economic Theory 7, 411-417.

Cumberland, J.H. and R.J. Korbach (1973), A Regional Interindustry Environmental Model, Papers of the Regional Science Association $30,61-75$.

Dieudonné, J. (1969), Foundations of Modern Analysis, Enlarged and Corrected Printing, New York u.a..

Fisher, A.C., J.V. Krutilla and C.J. Cicchetti (1972), The Economics of Environmental Preservation: A Theoretical and Empirical Analysis, American Economic Review 62, 605-619.

Fisher, A.C. and F.M. Peterson (1976), The Environment in Economics: A Survey, Journal of Economic Literature 14, 1-33.

Forster, B.A. (1973), Optimal Consumption Planing in a Polluted Environment, Economic Record 49, 534-545.

Forster, B.A. (1977a), On a One state Variable Optimal Control Problem, Consumption-Pollution Trade-Offs, in: J.D. Pitchford and S.J. Turnovsky (1977), Application of Control Theory to Economic Analysis, Amsterdam, 35-56.

Forster, B.A. (1977b), Pollution Control in a Two-Sector Dynamic General Equilibrium Model, Journal of Environmental Economics and Management 4, 305-312.

Fфrsund, F.R. (1979), Dynamic Aspects of Regional Environmental Policy, in Siebert et al. (1979).

Frey, R.L. (1979), Interregional Welfare Comparisons and Environmental Pclicy, in: Siebert et al. (1979).

Gale, D. and H. Nikaido (1965), The Jacobian Matrix and Global Univalence of Mappings, Mathematische Annalen 159, 81-93.

Hadley, G. and M.C. Kemp (1971), Variational Methods in Economics, Amsterdam.

Herfindahl, C.C. and A.V. Kneese (1974), Economic Theory of Natural Fiesources, Columbus.

Isard, W. (1972), Ecological-Economic Analysis for Regional Development, New York 
Isard, W. and P. Liossatos (1979), Spatial dynamics and optimal space-time development, Amsterdam.

Keeler, E., M. Spence and R. Zeckhauser (1972), The Optimal Control of Pollution, Journal of Economic Theory, 19-34.

Krutilla, J.V. and A.C. Fisher (1975), The Economics of Natural Environments, Baltimore.

Kydland, F. (1975), Noncooperative and Dominant Player Solutions in Discrete Dynamic Games, International Economic Review 16, $321-335$.

Mäler, K.G. (1974), Environmental Economics. A Theoretical Inquiry, Baltimore, London.

Nijkamp, P. (1978), Competition among Regions and Environmental Quality, in: W. Buhr und P. Friedrich (1978), Konkurrenz zwischen kleinen Regionen, Baden-Baden, 153-171.

Page, T. (1977), Conservation and Economic Efficiency. An Approach to Material Policy, Baltimore.

Pethig, R. (1975), Umweltverschmutzung, Wohlfahrt und Umweltpolitik in einem Zwei-Sektoren-Gleichgewichtsmodell, Zeitschrift für Nationalökonomie 35, 99-124.

Siebert, H. (1975a), Externalities, Environmental Quality and Allocation, Zeitschrift für Wirtschafts- und Sozialwissenschaften 1, 17-32.

Siebert, H. (1975b), Regional Aspects of Environmental Allocation, Zeitschrift für die Gesamte Staatswissenschaft 131, 496-513.

Siebert, H. (1978a), Ökonomische Theorie der Umwelt, Tübingen.

Siebert, H. (1978b), Regional Planning- Land Use Approaches to Environmental Quality Management, in: Proceedings of the Seminar on Environmental Pollution Control in the Context of Regional Planning, Katowice (Polen), 85-99.

Siebert, H. (1979) (Hrsg.), Umwelt und wirtschaftliche Entwicklung, Darmstadt.

Siebert, H., J. Eichberger, R. Gronych und R. Pethig (1980), Trade and Environment, Amsterdam.

Siebert, H., I. Walter und K. Zimmermann (1979), Regional Environmental Policy, Berlin.

Takayama, A. (1974), Mathematical Economics, Hinsdale, Illinois.

Tietenberg, T.H. (1973), Specific Taxes and the Control of Pollution; A General Equilibrium Analysis, Quarterly Journal of Economics 8, 503-522.

Umweltgutachten 1978, Stuttgart, Mainz.

Van Zele, R. (1978), An International (Interjurisdictional) Analytical Framework for Environmental Management, New York.

Vogt, W. (1981), Zur intertemporal wohlfahrtsoptimalen Nutzung knapper natürlicher Ressourcen. Eine kontrolltheoretische Analyse, Tübingen.

Wloka, J. (1971), Funktionalanalysis und Anwendungen, Berlin, New York.

Wright, C. (1974), Some Political Aspects of Pollution Control, Journal of Environmental Economics and Management 1, 173-186. 


\section{STAATLICHE ALLOKATIONSPOLITIK IM}

MARKTWIRTSCHAFTLICHEN SYSTEM

Band 1 Horst Siebert: Umweltallokation im Raum. 1982.

Band 2 Horst Siebert: Global Environmental Resources. The Ozone Problem. 1982.

Band 3 Hans-Joachim Schulz: Steuerwirkungen in einem dynamischen Unternehmensmodell. Ein Beitrag zur Dynamisierung der Steuerüberwälzungsanalyse. 1981 . 
Horst Siebert - 978-3-631-75607-2

Downloaded from PubFactory at 01/11/2019 03:09:22AM

via free access 